\title{
High resolution scattering spectropolarimetry of the quiet solar photosphere
}

\author{
Dissertation \\ zur Erlangung des mathematisch-naturwissenschaftlichen Doktorgrades \\ "Doctor rerum naturalium" \\ der Georg-August-Universität Göttingen \\ im Promotionsprogramm PROPHYS \\ der Georg-August University School of Science (GAUSS)
}

vorgelegt von

Franziska Zeuner

aus Bielefeld, Germany

Göttingen, 2020 
Betreuungsausschuss

Prof. Dr. Sami K. Solanki

Max-Planck-Institut für Sonnensystemforschung, Göttingen, Germany

Prof. Dr. Ansgar Reiners

Institut für Astrophysik, Georg-August-Universität Göttingen, Germany

Dr. Alex Feller

Max-Planck-Institut für Sonnensystemforschung, Göttingen, Germany

Dr. Michiel van Noort

Max-Planck-Institut für Sonnensystemforschung, Göttingen, Germany

Mitglieder der Prüfungskommision

Referent: Prof. Dr. Ansgar Reiners

Institut für Astrophysik, Georg-August-Universität Göttingen, Germany

Korreferent: Prof. Dr. Sami K. Solanki

Max-Planck-Institut für Sonnensystemforschung, Göttingen, Germany

Weitere Mitglieder der Prüfungskommission:

Prof. Dr. Ariane Frey

II. Physikalisches Institut, Georg-August-Universität Göttingen, Germany

Dr. Natalie A. Krivova

Max-Planck-Institut für Sonnensystemforschung, Göttingen, Germany

Prof. Dr. Hardi Peter

Max-Planck-Institut für Sonnensystemforschung, Göttingen, Germany

Prof. Dr. Karl-Henning Rehren

Institut für theoretische Physik, Georg-August-Universität Göttingen, Germany

Tag der mündlichen Prüfung: 26.08.2020 
"When we are chafed and fretted by small cares, a look at the stars will show us the littleness of our own interests." Maria Mitchell (1866) 


\section{Bibliografische Information der Deutschen Nationalbibliothek}

Die Deutsche Nationalbibliothek verzeichnet diese Publikation in der Deutschen Nationalbibliografie; detaillierte bibliografische Daten sind im Internet über http: //dnb . d-nb . de abrufbar.

(C) Franziska Zeuner

(c) (i) ()$_{\mathrm{BY}} \mathrm{N}_{\mathrm{ND}}$
Creative Commons Attribution 4.0 License

Printed in Germany 


\section{Contents}

$\begin{array}{lr}\text { List of Symbols } & \mathbf{8}\end{array}$

$\begin{array}{ll}\text { Abstract } & 9\end{array}$

$\begin{array}{ll}\text { Kurzfassung } & 13\end{array}$

$\begin{array}{llr}1 & \text { Introduction } & 17\end{array}$

2 Background $\quad \mathbf{2 1}$

2.1 The Sun's small-scale turbulent magnetic field . . . . . . . . . . . . . . 22

2.2 Light-matter interaction in the solar atmosphere . . . . . . . . . . . . 28

2.2.1 Polarization: Stokes formalism . . . . . . . . . . . . 28

2.2.2 The radiative transfer equation . . . . . . . . . . . . . . . 29

2.2.3 Absorption, emission and the atomic density operator . . . . . . . 30

2.3 Solar generation of spectral line polarization in $\mathrm{Sr}$ I . . . . . . . . . . . 33

2.3.1 The Zeeman effect . . . . . . . . . . . . . . . . . . . . 34

2.3.2 Scattering polarization ................. 38

2.3.2.1 Scattering polarization at the limb: Sr I in the second solar spectrum . . . . . . . . . . . . . 42

2.3.2.2 Scattering polarization at solar disk center . . . . . . 44

2.3.2.3 The Hanle effect . . . . . . . . . . . . . . . . . . 48

2.4 Ground-based fast solar spectropolarimetry . . . . . . . . . . . . 52

2.4.1 Stokes polarimetry by temporal modulation . . . . . . . . . . 52

2.4.2 The Fast Solar Polarimeter . . . . . . . . . . . . . . . . 54

2.4.3 The Fast Solar Polarimeter 2 . . . . . . . . . . . . . . 55

2.4.3.1 Dual-beam polarimetry . . . . . . . . . 55

2.4.3.2 Correction of intensity cross-talk . . . . . . . . 62

2.4.4 Fabry-Pérot interferometer based wavelength discrimination . . . 64

3 Detection of spatially structured scattering polarization of Sr I $4607.3 \AA$ with the FSP

3.1 Abstract .............................. 67

3.2 Introduction . . . . . . . . . . . . . . . . 68

3.3 Observations and data reduction . . . . . . . . . . . . . . 69

3.3.1 Center-to-limb variation of scattering polarization . . . . . . . . 69

3.3.1.1 Observations . . . . . . . . . . . . . 69

3.3.1.2 Data reduction . . . . . . . . . . . 70

3.3.2 Spatially resolved Stokes measurements . . . . . . . . . . . . 70

3.3.2.1 Observations . . . . . . . . . . . . 70 
3.3.2.2 Data reduction . . . . . . . . . . . 71

3.4 Results . . . . . . . . . . . . . . . . . . 72

3.4.1 Center-to-limb variation of scattering polarization . . . . . . . 72

3.4.2 Spatially resolved Stokes measurements . . . . . . . . . . . 75

3.4.2.1 Correlations with the continuum intensity . . . . . 75

3.4.2.2 Doppler-shift induced horizontal signal fluctuations . . 76

3.4.2.3 Cross-talk from Stokes I . . . . . . . . . . . . . 78

3.4.2.4 Influence of spatial resolution . . . . . . . . . 78

3.4.2.5 Estimation of polarimetric structure size . . . . . . 78

3.5 Discussion and conclusions . . . . . . . . . . . . . . . 82

4 Solar disk center shows scattering polarization in the $\mathrm{Sr}_{\mathrm{I}} 4607 \AA$ line $\quad \mathbf{8 5}$

4.1 Abstract . . . . . . . . . . . . . . . . 85

4.2 Introduction . . . . . . . . . . . . . . . . . 86

4.3 Observational data . . . . . . . . . . . . . . . . 87

4.4 Pixel classification based on the local quadrupole of the radiation . . . . . 88

4.5 Results . . . . . . . . . . . . . . . . . . . . 90

4.6 Discussion and conclusion . . . . . . . . . . . . . . . . 94

4.7 Appendix to Paper II . . . . . . . . . . . . . . . . . . . . . . . . . . 95

4.7.1 Observation . . . . . . . . . . . . . . 95

4.7 .2 Data reduction . . . . . . . . . . . . . . . 97

$4.7 .3 \quad J_{2}^{2}$ maps . . . . . . . . . . . . . . . . . . . . . . . . . . . . . . . . . .

5 Sr I $4607 \AA$ line at solar disk center: comparison between observation and $\begin{array}{ll}\text { simulation } & 101\end{array}$

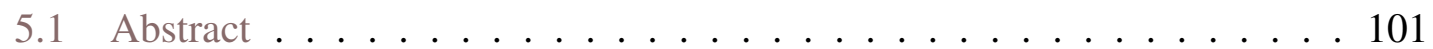

5.2 Introduction . . . . . . . . . . . . . . . . . . . 102

5.3 Observation and simulation data description . . . . . . . . . . 103

5.4 Results . . . . . . . . . . . . . . . . . . 106

5.4.1 Quantitative comparison between simulation and observation . . . 106

5.4.2 Reliability of reconstruction . . . . . . . . . . . . 109

5.4.3 Influence of solar temporal evolution . . . . . . . . . . . . 110

5.5 Discussion and conclusion . . . . . . . . . . . . . . . 112

6 Summary and outlook $\quad \mathbf{1 1 5}$

$\begin{array}{ll}\text { A Geometrical tensor } & \mathbf{1 2 3}\end{array}$

$\begin{array}{ll}\text { B Line profile } & \mathbf{1 2 5}\end{array}$

$\begin{array}{lrr}\text { C Supplementary material related to Paper II } & 127\end{array}$

C.1 Fitting surfaces and analytic expressions . . . . . . . . . . . . . . . 127

C.2 Time evolution of the radiation field coherence . . . . . . . . . 131

$\begin{array}{ll}\text { Bibliography } & 135\end{array}$

$\begin{array}{ll}\text { Acknowledgements } & 143\end{array}$

$\begin{array}{ll}\text { Curriculum vitae and publications } & 145\end{array}$ 


\section{List of Symbols}

\begin{tabular}{|c|c|}
\hline$\alpha$ & Polarizer angle \\
\hline$a$ & Camera non-linearity coefficient \\
\hline$A_{u \ell}$ & Einstein coefficient for spontaneous upper level decay \\
\hline$b$ & Camera linearity coefficient \\
\hline$B$ & Magnetic field \\
\hline$B_{\ell u}$ & Einstein coefficient for lower level excitation \\
\hline$c$ & Speed of light \\
\hline$\delta$ & Telescope induced polarization offset \\
\hline$\delta$ & Collision rate \\
\hline$D$ & Demodulation matrix \\
\hline$e$ & Electron charge \\
\hline$f$ & Focal length \\
\hline$f$ & Intensity cross-talk coefficient \\
\hline$f_{s}$ & Intensity cross-talk running coefficient \\
\hline$f_{B}$ & Magnetic filling factor \\
\hline$F$ & Finesse \\
\hline$\gamma$ & Line broadening parameter \\
\hline$g$ & Lande-factor \\
\hline$h$ & Planck constant \\
\hline $\mathcal{H}$ & Hanle depolarization coefficient \\
\hline $\mathrm{i}$ & Imaginary number \\
\hline$\epsilon_{I_{i}}$ & Emissivity per Stokes parameter \\
\hline$I, Q, U, V$ & Stokes parameters \\
\hline$I_{i}$ & Stokes parameters, with $i=0, . ., 3, I_{0}=I ; I_{1}=Q ; I_{2}=U ; I_{3}=V$ \\
\hline$J_{\ell}$ & Total angular momentum of lower level \\
\hline$J_{u}$ & Total angular momentum of upper level \\
\hline$J(\lambda)$ & Mean intensity \\
\hline$J_{q}^{k}$ & Multipole components of the radiation field \\
\hline$k$ & Tensor rank \\
\hline$\lambda$ & Wavelength in $\mathrm{nm}$ \\
\hline$\lambda_{0}$ & Central wavelength of spectral line \\
\hline$L$ & Geometrical distance \\
\hline$m$ & Zeeman sub-level \\
\hline$m_{e}$ & Electron mass \\
\hline$\mu$ & Limb distance \\
\hline$M$ & Optical magnification factor \\
\hline$\eta_{I_{i}}$ & Absorptivity per Stokes parameter \\
\hline$v_{L}$ & Larmor frequency \\
\hline$n_{\ell}$ & Lower level density \\
\hline$n_{u}$ & Upper level density \\
\hline$N$ & Total number of atoms \\
\hline
\end{tabular}




$\begin{array}{ll}\Omega & \text { Direction } \\ O & \text { Modulation matrix } \\ \varphi & \text { Azimuth angle } \\ \phi\left(\lambda-\lambda_{0}\right) & \text { Spectral line profile } \\ \psi & \text { Dispersion profile } \\ \Phi_{q}^{k k^{\prime}}\left(J_{\ell}, J_{u} ; \lambda\right) & \text { Generalized spectral line profile } \\ \rho\left(m, m^{\prime}\right) & \text { Atomic density matrix in the Zeeman basis } \\ \rho_{q}^{k} & \text { Atomic density matrix in spherical representation } \\ \rho_{Q, U, V} & \text { Dispersion } \\ r & \text { Photon shot noise } \\ R & \text { Glass reflectivity } \\ R^{2} & \text { Extinction ratio of a polarizer } \\ q & \text { Tensor component } \\ \tau_{\lambda} & \text { Optical depth } \\ \theta & \text { Polar angle } \\ \mathcal{T} & \text { Geometrical tensor } \\ X / I & \text { Place holder for normalized polarimetric Stokes parameters } \\ Y_{q}^{k}(\Omega) & \text { Spherical harmonics component }\end{array}$




\section{Abstract}

In this thesis $\mathrm{we}^{1}$ investigate small-scale scattering polarization signals in quiet, internetwork (IN) regions of the lower solar atmosphere. Small-scale polarization signals are inherently hard to observe due to their small spatial scale structuring with low amplitude and probably fast changing nature. We aim to measure and understand the horizontal fluctuations of these polarization signals, as this is the first step towards spatially resolved Hanle effect observations. Hanle effect observations are a promising complementary diagnostic tool for highly tangled and dynamic magnetic fields, which are invisible to standard Zeeman effect based observations. Therefore, Hanle effect observations have the potential to shed light on the small-scale dynamo action on the Sun's surface. But even if the scattering signals are not explicitly interpreted in terms of the Hanle effect, physical insights can still be gained into the formation and modification of the scattering polarization.

We use high spatial and temporal resolution observations, obtained by two groundbased polarimeters: the Fast Solar Polarimeter prototype (FSP) and an updated and modified version, FSP 2, to address the challenges of high polarimetric sensitivity in combination with high spatio-temporal resolution. We concentrate on the statistical analysis of the scattering polarization of the prominent Sr I spectral line in magnetically quiet solar regions. Some part of the analysis is performed using a novel method we developed to classify image pixels and average them according to the inhomogeneity of the solar granulation. This inhomogeneity breaks the axial symmetry of the radiation field, which was predicted to cause scattering polarization at solar disk center. Within this context, we have made an original contribution to knowledge in three areas: We produced the first published filtergraph observation of scattering polarization in Sr I where we found an anti-correlation between linear polarization and continuum intensity; we found the first significant evidence of spatially structured scattering polarization in Sr I at disk center; and we developed a new tool which opens possibilities to analyze observations of scattering polarization, even in low signal-to-noise regimes and compare them to numerical models.

In the first part of the thesis, we give an overview of the small-scale turbulent magnetic field on the Sun and polarizing mechanisms in the solar photosphere. Within the general theoretical framework of polarized spectral lines provided by Landi Degl'Innocenti \& Landolfi (2004) we focus on how scattering polarization emerges from anisotropic radiation and how this can be formally described. In doing so, we will not only cover the well known case for scattering polarization of spectral lines at the solar limb, but also at solar disk center.

\footnotetext{
${ }^{1}$ I will use "we" throughout my thesis to remind the reader that especially the results of the main part have been developed in cooperation with collaborators.
} 
To motivate how scattering polarization can be applied in astrophysical diagnostics, we briefly elaborate on the difference between the two main magnetic field diagnostic techniques, the Hanle and the Zeeman effect. We discuss why the Hanle effect, which modifies scattering polarization, is more suitable for diagnosing small-scale turbulent magnetic fields.

In the second part of the thesis, we discuss a filtergraph observation at the German Vacuum Tower Telescope on Tenerife carried out with the FSP. We present the observational evidence of spatially structured scattering polarization. The scattering polarization is structured with respect to intergranules and granules. Our statistical analysis reveals that the linear polarization component parallel to the north solar limb in the $\mathrm{Sr}$ I line core anti-correlates with the continuum intensity. Furthermore, we found that the spatial dimension of these structures are on the order of $0.5^{\prime \prime}-1^{\prime \prime}$. We show that the polarization signals are consistent with numerical models. These models suggest that the polarization predominantly emerges from intergranules, i.e. more precisely, from the interface between intergranules and granules, due to local radiation anisotropy. This finding, however, contradicts the result of two studies that were conducted earlier (Malherbe et al. 2007; Bianda et al. 2018). Especially these studies were carried out with spectrographs and at a solar limb distance of $\mu=0.3$, while our filtergraph observation was done at $\mu=0.6$.

In the third part of the thesis, we study the linear polarization signals in the Sr I line at solar disk center observed with FSP 2 at the Dunn Solar Telescope, New Mexico. Due to geometric considerations, the mean scattering polarization should be zero. However, we found polarization signals which correlate with the local axial symmetry break of the radiation field. As a zero reference, we observed the continuum and a neighboring Fe I spectral line position. The latter spectral line serves as a zero reference, as it is insensitive to scattering. We introduce a statistical method that allows for the first time to show horizontally fluctuating scattering polarization in both linear polarization states. We are even able to convert the statistical values back to images and roughly reconstruct the linear polarization maps. As we used a medium sized solar telescope, this opens the unexpected opportunity to study these signals already in currently available solar observatories, where signal-to-noise ratios are not sufficient to detect such small-scale scattering signals directly. These observational results support a range of theoretical predictions (del Pino Alemán et al. 2018). Our findings support the picture of an isotropic magnetic field in the quiet Sun, with magnetic field amplitudes small enough to safely stay away from the Hanle saturation regime ( 100 G for Sr I).

Our analysis furthermore supports and further restricts the findings from the second part of the thesis. We find that the mean spatial size for scattering polarization in this observation is about $0.75^{\prime \prime}$, but larger structures $\left(\sim 2^{\prime \prime}\right)$ are visible, too.

Finally, we compare the results found in the third part with a published state-of-the-art 3D-MHD simulation snapshot, where the Sr I line has been synthesized using the 3D radiative transfer code PORTA (del Pino Alemán et al. 2018). The magnetic field and collisional rates have been chosen to reproduce center-to-limb observations of Sr I. We degrade the synthesized data to the FSP 2 observations. Our analysis of the comparison between the simulation and the observation reveals very interesting findings on two levels. Firstly, we are able to test the statistical method introduced in the second part by applying it to noisy and noise-free simulation data and find that it is more reliable for structures larger than 1 ". This preliminary result implies that the size estimation in the third part of 
this thesis may be biased towards larger structures. Secondly, the spatial distribution of the scattering polarization in the reconstructed observation is comparable to the simulation. However, the polarization amplitude in the observation is reduced by a factor of two compared to the simulation. We find indications that temporal evolution of the Sun is the main cause of this reduction of the observed scattering polarization amplitude, not additional magnetic fields. This may indicate that the simulated photosphere is magnetized in the right amount to mimic the "true" photosphere, which means that the solar lower atmosphere may be magnetized close to equipartition. Two other (but less probable) possibilities are that either the depolarizing collision rates are much higher than expected by center-to-limb observations of Sr I or/and that the small-scale, yet undetected magnetic field is even stronger and/or more dynamic in the Sun than is suggested by center-to-limb observations of $\mathrm{Sr}$. The latter case, if it proves to be true, implies that there is more magnetic energy hidden in the Sun than was previously expected. 



\section{Kurzfassung}

In der vorliegenden Arbeit bieten wir Lösungsansätze für die Problematik, hochgradig dynamische und kleinskalige Streupolarisation in den ruhigen Regionen der unteren Sonnenatmosphäre zu untersuchen. Solche kleinskaligen Signale sind grundsätzlich schwer zu beobachten, da sie niedrige Amplituden auf kleinen räumlichen Skalen aufweisen und möglicherweise schnellen Veränderungen unterliegen. Unser Ziel ist es, die horizontalen Fluktuationen der Streupolarisation zu beobachten und zu verstehen, da dies ein Schritt in Richtung räumlich aufgelöster Hanle-Effekt Beobachtungen ist. Hanle-Effekt Beobachtungen sind ein vielversprechendes, komplementäres Instrument, um sehr chaotische und dynamische magnetische Felder auf der Sonne zu messen, die für Standardbeobachtungen basierend auf dem Zeeman-Effekt unsichtbar sind. Damit machen Hanle-Effekt Beobachtungen potentiell den kleinskaligen Dynamo zugänglich, der auf der Sonnenoberfläche operiert. Aber selbst wenn die Streusignale nicht explizit mit Blick auf den Hanle-Effekt interpretiert werden, können trotzdem physikalische Einsichten über die Entstehung und Veränderung der Streupolarisation gewonnen werden.

Wir verwenden Beobachtungen mit hoher räumlicher und zeitlicher Auflösung, die mit zwei bodengebundenen Polarimetern gewonnen wurden: dem Prototyp des Fast Solar Polarimeter (FSP) und einer aktualisierten und modifizierten Version von FSP, genannt FSP 2, um die Herausforderungen der hohen polarimetrischen Empfindlichkeit in Kombination mit hoher räumlich-zeitlicher Auflösung anzugehen.

Wir konzentrieren uns auf die statistische Analyse der Streupolarisation der prominenten Sr I Spektrallinie in magnetisch ruhigen Sonnenregionen. Ein Teil der Analyse wird mit einer neuartigen Methode durchgeführt, die wir entwickelt haben, um Bildpunkte entsprechend der Inhomogenität des Strahlungsfeldes, gegeben durch die solare Granulation, zu klassifizieren und zu mitteln. Die Inhomogenität bricht die axiale Symmetrie des Strahlungsfeldes, welche die Streupolarisation im Zentrum der Sonnenscheibe überhaupt erst verursacht. In diesem Zusammenhang haben wir einen originellen Beitrag zum Wissen in drei Bereichen geleistet: die erste publizierte Filtergraph-Beobachtung der Streupolarisation in Sr I, in der wir eine Anti-Korrelation zwischen linearer Polarisation und Kontinuumsintensität fanden; die erste signifikante Evidenz für räumlich strukturierte Streupolarisation in Sr I im Zentrum der Sonnenscheibe; und die Entwicklung einer neuen Methode, die die Möglichkeiten eröffnet, Streupolarisationsbeobachtungen auch im Falle eines kleinen Signal-zu-Rausch-Verhältnisses zu analysieren und mit numerischen Modellen zu vergleichen.

Im ersten Teil dieser Dissertation geben wir einen Überblick über das kleinskalig turbulente Magnetfeld auf der Sonne und über die Polarisierungsmechanismen in der Photosphäre der Sonne. Basierend auf der generellen theoretischen Beschreibung von Polarisation in Spektrallinen von Landi Degl'Innocenti \& Landolfi (2004) beschreiben wir 
(auch formal), wie Streupolarisation bei anisotroper Strahlung entsteht. Die Beschreibung beinhaltet nicht nur den sehr bekannten Fall von Streupolarisation von Spektrallinien am Sonnenrand, sondern auch in der Sonnenmitte. Wir erarbeiten den Unterscheid zwischen den zwei hauptsächlich verwendeten Techniken zur Magnetfelddiagnostik, dem Hanle und dem Zeeman-Effekt, und weshalb der Hanle-Effekt sich eher für kleinskalige turbulente Magnetfelder eignet.

Im zweiten Teil der Dissertation werden wir eine Filtergraph-Beobachtung am deutschen Vakuumturm-Teleskop auf Teneriffa besprechen, die mit dem FSP durchgeführt wurde. Wir präsentieren den Nachweis einer räumlich strukturierten Streupolarisation. Die Streupolarisation ist in Bezug auf Intergranulen und Granulen strukturiert. Unsere statistische Analyse ergibt, dass die lineare Polarisationskomponente parallel zum solaren Nordrand im Kern der Sr I Linie mit der Intensität des Kontinuums antikorreliert. Darüber hinaus stellen wir fest, dass die räumliche Dimension dieser Strukturen in der Größenordnung von $0,5^{\prime \prime}-1^{\prime \prime}$ liegt. Wir zeigen, dass die Polarisationssignale mit numerischen Modellen übereinstimmen. Diese Modelle deuten darauf hin, dass die Polarisation aufgrund der lokalen Strahlungsanisotropie überwiegend aus Intergranulen (genauer gesagt aus der Grenzfläche zwischen Intergranulen und Granulen) resultiert. Diese Erkenntnis widerspricht dem Ergebnis von zwei früheren Studien (Malherbe et al. 2007; Bianda et al. 2018). Diese Studien wurden jedoch mit Spektrographen und bei einem Sonnenrandabstand von $\mu=0,3$ durchgeführt, während unsere Filtergraph-Beobachtung bei $\mu=0,6$ durchgeführt wurde.

Im dritten Teil der Dissertation untersuchen wir die linearen Polarisationssignale in der Sr r Linie im Zentrum der Sonnenscheibe, die mit FSP 2 am Dunn Solar Telescope, New Mexico, beobachtet wurden. Aus geometrischen Gründen sollte die mittlere Streupolarisation Null sein. Wir fanden jedoch Polarisationssignale, die mit dem lokalen axialen Symmetriebruch des Strahlungsfeldes korrelieren. Als Testfälle haben wir das Kontinuum und die benachbarte Fe I Spektrallinienposition beobachtet, welche unempfindlich sind gegenüber Streupolarisation. Wir stellen eine statistische Methode vor, die es erstmals ermöglicht, horizontal flukturierende Streupolarisation in beiden linearen Polarisationszuständen nachzuweisen. Wir sind sogar in der Lage, lineare Polarisationskarten grob zu rekonstruieren, wobei die statistischen Werte in Polarisationskarten umgewandelt werden. Da wir ein mittelgroßes Sonnenteleskop verwendet haben, eröffnet sich dadurch die unerwartete Möglichkeit, diese Signale bereits mit derzeit verfügbaren Sonnenobservatorien zu untersuchen, wo das Signal-zu-Rausch-Verhältnis nicht ausreicht, um Streusignale direkt zu erfassen. Diese Beobachtungsergebnisse unterstützen eine Reihe von theoretischen Vorhersagen (del Pino Alemán et al. 2018). Unsere Ergebnisse unterstützen das Bild eines isotropen Magnetfeldes in der ruhigen Sonne, mit Magnetfeldamplituden, die klein genug sind, um sich sicher vom Hanle-Sättigungsregime fernzuhalten ( 100 G für Sr I).

Unsere Analyse unterstuitzt die Erkenntnis aus dem zweiten Teil der Dissertation und schränkt sie weiter ein. Wir haben herausgefunden, dass die gemittelte räumliche Ausdehnung für die Streupolarisation in dieser Beobachtung ungefähr bei 0,75" liegt, wobei auch größere Strukturen durchaus vorhanden sind.

Schlußendlich vergleichen wir die Ergebnisse des dritten Teils mit einer aktuellen 3DMHD-Simulationsmomentaufnahme, bei der die Sr I Linie mit dem 3D-Strahlungstransportcode PORTA synthetisiert wurde (del Pino Alemán et al. 2018). Das Magnetfeld und die Kollisionsraten wurden so gewählt, dass Mitte-zu-Rand-Beobachtungen von Sr I re- 
produziert werden können. Wir reduzieren die räumliche und spektrale Auflösung der synthetisierten Daten auf die FSP 2 Beobachtungen. Unsere Analyse des Vergleichs zwischen Simulation und Beobachtung zeigt sehr interessante Ergebnisse auf zwei Ebenen. Erstens können wir die im dritten Teil vorgestellte statistische Methode testen, indem wir sie auf verrauschte und rauschfreie Simulationsdaten anwenden und feststellen, dass sie zuverlässiger für Strukturen größer als 1" funktioniert. Zweitens, die räumliche Verteilung der Streupolarisation in der rekonstruierten Beobachtung ist vergleichbar mit der Simulation. Die Polarisationsamplitude in den Beobachtungen ist jedoch um den Faktor zwei reduziert, verglichen mit der Simulation. Wir finden Hinweise, dass die zeitliche Entwicklung der Sonnenoberfläche die Hauptursache für die beobachtete reduzierte Streupolarisationsamplitude darstellt, nicht zusätzliche magnetische Felder. In diesem Fall wäre die Sonnenoberfläche etwa bis zur Gleichverteilung magnetisiert. Zwei andere (wenn auch weniger wahrscheinliche) Möglichkeiten sind, dass entweder die depolarisierende Kollisionsraten viel höher sind als von Mitte-zu-Rand-Beobachtungen erwartet oder/und dass das kleinskalige, aber beobachterisch nicht direkt erfasste Magnetfeld in der Sonne sogar noch stärker und/oder dynamischer ist, als es von Mitte-zu-Rand-Beobachtungen von Sr I angedeutet wird. Der letzte Fall, wenn er sich als belastbar erweist, impliziert, dass in der Sonne mehr magnetische Energie verborgen ist, als bis jetzt erwartet wurde. 



\section{Introduction}

Whenever we look at the stars, what we perceive with our eyes is dominated by the surface of these giant balls of plasma. The same is true for the star that sits in the center of our solar system - although the proximity to us is a potential danger for our eyes if we look at it directly. The surface of the Sun delineate the lowest part of the solar atmosphere, called the photosphere. While only covering about less than $0.1 \%$ of the Sun's full diameter, it may be easy to underestimate the significance of the photosphere's role for the understanding of our host star. The photosphere is the lowest layer of the solar atmosphere which we can directly observe using the visible part of the electromagnetic spectrum. The electromagnetic waves in the form of light leaving the Sun's surface had a very long journey: they started as high energy x-ray quanta in the core of the Sun and lost most of their energy while scattering their way outwards to less dense and cooler plasma regions, until they reach the photosphere. Here, the optical density of the plasma is decreased to the level where it gets transparent (Stix 2004). The major part of the visible and infrared light originates in the photosphere. Therefore, and thanks to the proximity to our Earth, the photosphere is the deepest layer where the Sun can actually be spatially resolved with some detail by modern ground and space-based, balloon-borne and rocket solar telescopes.

Like the Earth's surface reacts to earthquakes, the photosphere is affected by what is happening underneath, while in turn effects what is above it. Similarly, the Earth's surface, for example, provides humidity and heat which affects the Earth's atmosphere in the form of storms. Thus, every detail that is not understood in the photosphere is a missed opportunity to understand more about the whole Sun. Not to mention that any insight into the photosphere of our Sun has also implications for our understanding of other (cool) stars, and stellar activity in general (e.g., review by Christensen-Dalsgaard 2020).

Historically, observations of the photosphere uncovered the most intriguing mechanisms operating in the Sun. For example, owing to dark sunspots appearing quasiperiodically on the surface, Samuel Heinrich Schwabe discovered the prominent 11-year solar cycle in 1843 from long-term observations of sunspots, which had been recorded since the 17 th century.

In addition to white light (continuum) observations, we are able to gain tremendous amount of information about the physics in the photosphere and the overlying layers (the chromosphere and also the corona) using remote spectropolarimetric observations. Spectropolarimetry enables us to capture the full information provided by light: its energy and its polarization. Considering the distance of the Earth to the Sun, 149 million kilometers or $1 \mathrm{AU}$ (astronomical unit), this is practically the only source of information we can rely on. In-situ measurements are so far only possible in the outer most layers of the corona and in the heliosphere filling the entire solar system. The closest-ever spacecraft, which is 
Parker Solar Probe (Fox et al. 2016), plans to get as close as nine solar radii in 2024.

Which kind of information can be stored in the light's energy-spectrum and its polarization?

The plasma of the Sun is composed, for the most part, of hydrogen. But many heavier elements are present, too. This has far-reaching implications. Given that the plasma of the photosphere is only partially ionized, re-combinations and atomic transitions form atomic and molecular spectral lines, once the temperature has dropped sufficiently. In fact, the photosphere covers about $600 \mathrm{~km}$ around $\log \left(\tau_{500}\right)=0\left(\tau_{\lambda}\right.$ is the optical depth), the upper part is marked by the effective ${ }^{1}$ temperature minimum of $\sim 5700 \mathrm{~K}$. Spectral lines, as well as the continuum, carry information about the physical conditions during their formation. Magnetic fields or velocity fields leave characteristic signatures in the shape and polarization of spectral lines, while the temperature defines, amongst others, the continuum intensity level.

The existence of spectral lines led to a groundbreaking discovery. Hale, who measured not only the spectrum but also the polarization state of the light of sunspots, confirmed his suspicion of a magnetic Sun (Hale et al. 1919). Later, it was discovered that, within the solar cycle, the Sun's activity increased during periods of higher sunspot occurrence. This was the beginning of intensive research on the interplay between the solar plasma and solar magnetic fields. What we know today is that this interplay is undeniably linked to all phenomena we commonly refer to as solar activity: from sunspot numbers to coronal mass ejections. Our interest in solar activity goes beyond pure scientific curiosity about our Sun. Solar activity is what directly affects the interplanetary environment (space weather) and thus the Earth's magnetosphere, atmosphere, and any human technology — but is still not fully understood (e.g., Haigh et al. 2004; Solanki et al. 2006; Riley et al. 2017; Archontis \& Vlahos 2019; Hapgood 2019, and references therein). Furthermore, what we learn about solar activity has implications on our knowledge of other stars and (a hot topic nowadays) exoplanets.

The magnetic field plays a key and diverse role within the solar activity. Its function ranges from energy storage, channelling plasma and fast charged particles, to producing instabilities and waves. In the literature, and from an observational point of view, astrophysical magnetic fields are typically decomposed into small- and large-scale. The large-scale magnetic field shows coherent structures of the dimensions of the object to which it is attributed. One example is the dipole-like solar magnetic field, which reverses its polarity during the 11-year solar cycle. The small-scale field, on the other hand, is linked to the turbulent motion of astrophysical plasma, like in the photosphere. Small-scale magnetic fields are found at spatial scales smaller than the initial turbulence driving range (e.g., Borrero et al. 2015; Schekochihin et al. 2004).

On the theoretical level, several dynamo mechanisms are known, which can explain how the magnetic field at these two scales might be generated (Brandenburg \& Subramanian 2005). However, if and to what extent any of these dynamo mechanisms operate in the Sun is still under debate (e.g., Moll et al. 2011; Rempel 2014). One especially challenging and interesting question is, if a small-scale solar surface dynamo found in simulations is present and observable in quiet solar surface regions (Vögler \& Schüssler 2007). ${ }^{2}$

\footnotetext{
1 Considering the Sun as a thermodynamic black body.

2 In fact, the existence of a small-scale dynamo depends strongly on the simulation's boundary conditions
} 
Quiet Sun regions are usually defined by a minimum of magnetic activity, in contrast to magnetically active regions, like sunspots. Along these lines another question is how strong the turbulent small-scale magnetic field actually is. The answer to this question has important implications on how much energy can be stored and transported within the solar atmosphere (Trujillo Bueno et al. 2004). At present, based on a comparison of spatially unresolved measurements to simulations, there is evidence that the photosphere is magnetized close to equipartition (del Pino Alemán et al. 2018), meaning that the kinetic energy balances the magnetic energy.

The most difficult problem is that small-scale magnetic activity is inherently hard to observe. This differentiates from the large-scale magnetic fields, which tend to be strong and have a sufficiently long lifetime to be easily observable with magnetographs and therefore have been studied extensively in the past. ${ }^{3}$ The term small-scale in this context is ambiguous in the literature. Usually, there is a distinction between small-scale concentrated (strong) and small-scale turbulent (and usually an order of magnitude weaker) magnetic fields (Bellot Rubio \& Orozco Suárez 2019).

Thanks to the Sun-Earth proximity, state-of-the-art solar telescopes provide resolution elements as small as $50 \mathrm{~km}$ and thereby the unique and special opportunity to study a star in a great detail. Despite this resolution, there has been observational evidence supported by magnetic field infused ${ }^{4}$ hydrodynamic simulations that magnetic energy is stored in a turbulent manner at scales even below the currently achievable instrumental resolution element (Trujillo Bueno et al. 2004). This discovery was made by comparing spatially unresolved spectropolarimetric observations of the photospheric Sr I spectral line, located at $4607.3 \AA$, with synthesized spectra while varying the magnetic field strength, where a certain magnetic field distribution varying with depth is assumed. The reason why this sub-resolution estimate was possible is because $\mathrm{Sr} \mathrm{I}$ is sensitive to the Hanle effect. In contrast to the routinely used Zeeman effect to remotely diagnose magnetic fields on the Sun (e.g., review by Solanki et al. 2006) and astrophysical plasma in general (e.g., Casini et al. 2008), the Hanle effect modifies spectral line polarization generated by scattering according to the unsigned amplitude of the magnetic field (Stenflo 1982). Therefore, the Hanle effect is resistant to cancellations of polarization signatures, if mixed polarity magnetic fields within the resolution element are present.

In recent years, there has been an increased interest in studying a spatially resolved Hanle effect in the Sr I line observationally (Malherbe et al. 2007; Bianda et al. 2018; Zeuner et al. 2018; Dhara et al. 2019; Zeuner et al. 2020) and theoretically (Trujillo Bueno \& Shchukina 2007; del Pino Alemán et al. 2018). Within the framework of this thesis, we focus on the analysis of high-resolution spectropolarimetric observations in the Sr I line with the Fast Solar Polarimeter attached to ground-based solar observatories. We show that the linear polarization is structured with respect to the well-known photospheric granulation structure. This work helps to improve the understanding of the polarizing scattering processes in the photosphere. It also provides to the solar community a novel method

(Vögler \& Schüssler 2007).

3 For example, sunspots and the polar magnetic field as a function of latitude over the solar cycle in the form of butterfly diagrams, which were originally proposed by Maunder (1904) (see also review by Hathaway 2015). These diagrams powerfully demonstrate some of the main features of large-scale magnetic fields over the solar cycle, like polar field reversals, Hale's polarity law and Joy's law.

4 The magnetic field was not part of the original simulation but was artificially added later. 
based on statistical averages over areas which a priori accommodate scattering polarization with a specific sign. This method allows for statistically analysis of spectropolarimetric observations which then can also be compared to simulations. As a consequence, this thesis brings us a step closer to the ultimate goal of spatially resolved Hanle observations. These types of observations have the potential to solve some problems in solar surface small-scale dynamo theory. One of the open questions is how the kinematic energy of the plasma is converted to magnetic energy, where spatially (and temporally) resolved Hanle observations give valuable insights. Besides the dynamic aspect of the problem, these observations also provide the opportunity to constrain the turbulent small-scale magnetic flux. The amount of magnetic flux has implications for the energy balance of the Sun and also for how much energy may be transported to the upper layers of the solar atmosphere where it contributes to heating the plasma.

Numerical models of the Sun's quiet surface help to understand these open questions, but they need to be consistent with observations in order to gain insight into the causes of discrepancies between the modeled and the real Sun. Only recently have observational and analytic techniques been made available to study small-scale polarization features, which give feedback to the decade old numerical prediction that scattering polarization signals in Sr I have a sub-granular structure (Trujillo Bueno et al. 2004) and therefore have the potential to one day diagnose magnetic fields at the smallest scales. ${ }^{5}$

In the next chapters we give a brief background on small-scale turbulent magnetic fields in the Sun. We show how they manipulate the polarization of spectral lines via the effects named after Hanle and Zeeman. We address how the polarization of light is generated in the solar atmosphere, how it is measured and how the information on the magnetic field can be extracted, whereby we outline how the Hanle effect is complementing the Zeeman effect when it comes to diagnosing tangled magnetic fields.

5 Snik et al. (2010) found evidence for sub-granular structure of scattering polarization in CN-band data provided by HINODE for limb distances up to $\mu=0.4$. 


\section{Background}

The Sun is a radiating plasma ball, covering many degrees of plasma ionization: from highly ionized in the core, to weakly ionized in the lower atmosphere, to again a highly ionized outer atmosphere. The plasma is constantly moving on different scales, from gigantic convection cells in the solar interior to small-scale turbulent convection at the surface. This dynamic ionized environment is an incubator for magnetic fields. However, observations indicate that the magnetic energy is independent of the spatial scales at which it is probably created and that the plasma itself is coupled to the magnetic field. This results in a highly complex and theoretically challenging situation, but gives also rise to numerous interesting phenomena - many of them remain poorly understood. One puzzling aspect is the origin and dynamics of small-scale turbulent magnetic fields on the surface of the Sun. A major reason for this lack of knowledge are observational challenges: the smaller the details to be resolved, the faster ${ }^{1}$ and more sensitive measurements have to become.

Although great work has been carried out in an effort to constrain the turbulent component of the small-scale magnetic fields, there are still many open questions. In this background section we motivate the work of this thesis by briefly explaining the open questions regarding the small-scale turbulent magnetic fields on the Sun. We also discuss a possible detection method for these types of magnetic fields based on the Hanle effect, which essentially represents a modification of scattering polarization in the presence of a magnetic field. We pay special attention to how scattering polarization is generated. Finally, we briefly describe how scattering polarization is measured in this thesis with the Fast Solar Polarimeter and the Fast Solar Polarimeter 2.

\footnotetext{
${ }^{1}$ Given a typical velocity of disturbances $\left(\approx 7 \mathrm{~km} \mathrm{~s}^{-1}\right)$ in the photosphere, the cadence has to decrease proportionally with the spatial scale in order to observe the disturbance.
} 


\subsection{The Sun's small-scale turbulent magnetic field}

The questions we address in this section are: What are small-scale turbulent magnetic fields on the Sun? What is known and what is unknown about them, and which role do they play on the Sun?

The answers to these questions help to contextualize and motivate the spectropolarimetric measurements presented in this thesis. Even if magnetic fields are not explicitly the main focus of this thesis, they motivate to study spectropolarimetric observations of scattering polarization. These observations have the potential to investigate magnetic fields on a presently unattainable spatio-temporal resolution scale.

At any time, even during periods of solar maximum, ${ }^{2}$ the largest fraction (at least $90 \%$ ) of the solar surface is covered by the so called quiet Sun. Figure 2.1 shows examples of magnetic fields during high (Figure 2.1 a) and low (Figure $2.1 \mathrm{~b}$ ) solar activity. The word quiet refers historically to regions on the Sun that are magnetically inconspicuous, in contrast to magnetically active regions, such as sunspots or plages. The latter show concentrations of magnetic fields up to a few $\mathrm{kG}$ and are highly associated with other phenomena such as flares and coronal mass ejections, to mention just a few. These phenomena are assigned to solar activity.

Photospheric quiet Sun areas are dominated by convective bright granules (rising hot plasma) and dark intergranules (down-flowing cold plasma), which appear in the continuum

${ }^{2}$ Phase of highest number of sunspots and highest activity during the solar cycle.

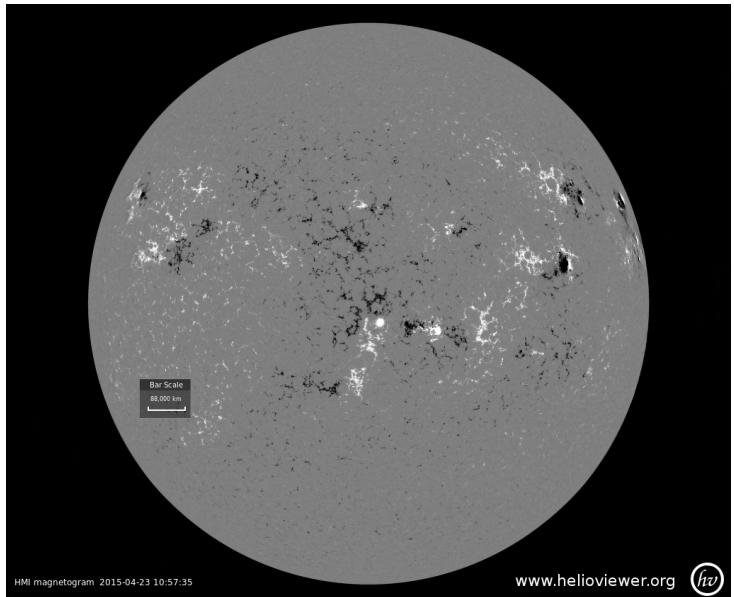

(a)

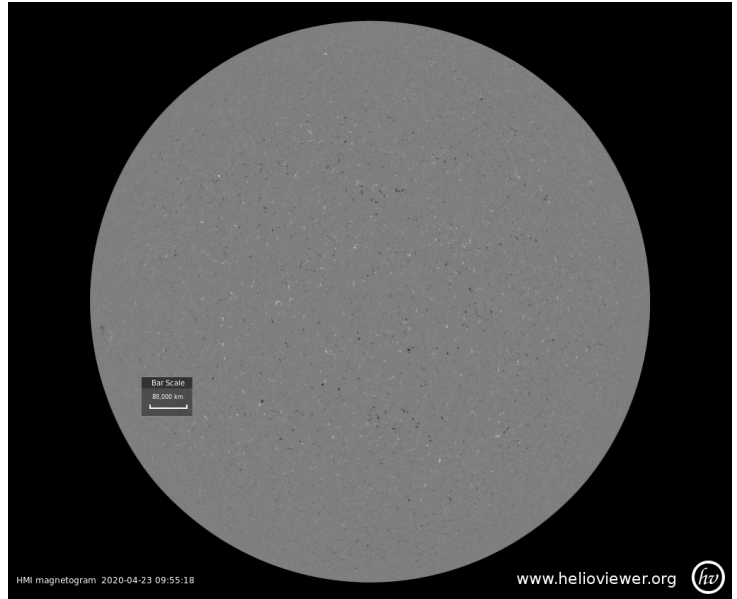

(b)

Figure 2.1: Magnetograms seen by the Helioseismic and Magnetic Imager (HMI, Schou et al. 2012) onboard the Solar Dynamics Observatory. Black and white denote opposite polarity magnetic fields. a) During high solar activity, strong magnetic field concentrations in the form of sunspots and plages are covering the solar disk. b) The magnetically quiet solar disk during low solar activity at the end of cycle 24 respectively the beginning of cycle 25. Concentrations of magnetic fields are marginally above the detection limit. Courtesy of NASA, ESA and JAXA. Images created using the ESA and NASA funded Helioviewer Project. 
intensity images (Bellot Rubio \& Orozco Suárez 2019, and references therein), such as in Figure 2.2 a).

The quiet Sun can be subdivided in the internetwork (IN) and the network. Although a strict definition is missing, the network is outlining the large scale convection cells (supergranulation) (e.g., de Wijn et al. 2009) with small-scale magnetic field concentrations in the $\mathrm{kG}$ range. On the other hand, typical quiet Sun regions are located within, that is outlined by, the magnetic network, the IN (for a brief review, see Solanki 2009). Until the beginning of the 1970s, the magnetic flux in the IN was believed to be much lower than in the network (or even absent): it was found by Howard \& Stenflo (1972) that a total of more than $90 \%$ of the magnetic flux of the solar surface is concentrated in network regions. However, over the past decades, with steadily improving solar observatories, new instrumental concepts and instruments with increasing sensitivity, the idea that there are non-magnetic solar regions was slowly disregarded. First evidence for quiet Sun IN magnetism was given by Livingston \& Harvey (1975). Since then, it has been argued that the IN may contain most of the existing unsigned magnetic flux on the surface at any given time (Stenflo \& Keller 1997; Trujillo Bueno et al. 2004; Sánchez Almeida 2005; Solanki et al. 2006). Observational evidence was given that the flux per element in the IN is about 1-2 orders of magnitude smaller than in the network, but the number of elements is so high that the total flux outnumbers the network flux. The lifetime of granules is short (a few minutes only, see Hirzberger et al. 1999), but allows a large amount of magnetic flux to be transported to the solar surface (Bellot Rubio \& Orozco Suárez 2019). Lagg et al. (2016) found that $80 \%$ of a quiet solar region observed in magnetically highly sensitive infrared Fe I lines show magnetic activity with high statistical significance above the noise level, where field strengths cover two orders of magnitude $(10 \mathrm{G}-\mathrm{kG})$.

The IN as well as the network magnetic field tend to accumulate in the down-flowing regions of the plasma, either the boundaries of supergranules or granules, e.g., de Wijn et al. (2009). However, while network magnetic fields are unipolar and mostly vertical with respect to the solar surface, IN magnetic fields present mixed polarity characteristics. For a long time it has been debated whether the vertical component dominates over the horizontal component or the magnetic field inclination is tropically distributed (Solanki et al. 2006). The latest results indicate that magnetic fields are more inclined the weaker they are, while strong fields tend to be vertical, for a comprehensive discussion on this topic see Bellot Rubio \& Orozco Suárez (2019). Extensive reviews on IN magnetic fields are given by de Wijn et al. (2009) and Bellot Rubio \& Orozco Suárez (2019).

Additionally, to the well-studied magnetic fields mentioned above, a turbulent component of the magnetic field was suggested to exist since the early 80s (Stenflo 1982). ${ }^{3}$ A turbulent component implies that azimuths and inclinations are randomly distributed within the resolution element (Bellot Rubio \& Orozco Suárez 2019). In the past three decades, there has been some observational evidence for the existence of a small-scale turbulent magnetic component below the resolution of observations (Faurobert-Scholl 1993; Trujillo Bueno et al. 2004; Graham et al. 2009; Danilovic et al. 2010b; Lites 2011). Moreover, works by e.g. Lites et al. (1996), Trujillo Bueno et al. (2004) and Lites (2011) provide evidence that the small-scale turbulent component is the dominant element of the total

\footnotetext{
${ }^{3}$ It has been suggested that the IN magnetic fields mentioned above are already part of the turbulent component (Brandenburg \& Subramanian 2005).
} 
magnetic energy in the quiet photosphere, and simulations by Rempel (2014) suggest that $50 \%$ of the energy resides at scales below $100 \mathrm{~km}$. One open question is (Graham et al. 2009; Bellot Rubio \& Orozco Suárez 2019): What is the probability distribution function for the turbulent magnetic field strengths in the Sun?

The probability density distribution determines especially the mean magnetic field strength. However, depending on the assumed specific shape of the distribution, the mean turbulent magnetic field strength varies in the literature, also because of observational biases (Domínguez Cerdeña et al. 2006). Usually, it is about $100 \mathrm{G}$ - probably more in the intergranules, and less in the granules (Trujillo Bueno et al. 2004). To summarize: we need spatially resolved observations of weak (compared to the network) turbulent magnetic fields. This would significantly improve our knowledge about the probability density function at smaller spatial scales (Bellot Rubio \& Orozco Suárez 2019). This again constraints the magnetic energy present on the smallest spatial scales, with far-reaching implications on the energy balance of the solar atmosphere (Trujillo Bueno et al. 2004).

One characteristic feature of a turbulent magnetic field is that the spatial scales at which the magnetic fields are tangled do not stop at the resolution of the telescopes, but cascade down to the scale of a few meters (Stenflo 2012a; Rempel 2014). There is indirect observational evidence for a maximum that can range from 1 to $10 \mathrm{~km}$ (Stenflo 2012b), which means that magnetic fields tangle at scales smaller than the horizontal mean free path of the photons, about $100 \mathrm{~km}$, therefore sometimes the term micro-turbulent is used. This unresolved magnetic field is hard to be observed with standard techniques such as the Zeeman effect. The Zeeman effect causes a spectral splitting of the differently polarized components of spectral lines. As we show in the next chapter, highly mixed polarity fields inside the resolution element escape detection with the Zeeman effect. On the other hand, the Hanle effect has the potential to complement Zeeman effect measurements in the weak, but turbulent magnetic field regime, see next chapter, and Stenflo \& Keller (1997), and Trujillo Bueno (2003a). The Hanle effect is a modification of scattering polarization of spectral lines. This modification is caused by weak magnetic fields, which may induce a polarization rotation and a depolarization of spectral line scattering polarization. Therefore, the Hanle effect is suitable for remote diagnostics of stellar magnetic fields. Moreover, the Hanle effect is sensitive to the unsigned magnetic flux. This qualifies the Hanle effect in particular for the study of turbulent magnetic fields beyond the resolution limit of solar telescopes. ${ }^{4}$ Therefore, the scientific community has agreed over the years that the study of the Hanle effect is the most promising (if not the key) tool for peering into such observationally challenging fields and investigating this aspect of the solar magnetism. We discuss this aspect in more detail in the next chapter.

To study the small-scale turbulent magnetic field in the photosphere the selection of spectral lines is relatively limited, see also next chapter. One important prerequisite for a suitable spectral line for Hanle effect diagnostics is a strong scattering polarization signal. A prominent spectral line, which meets this requirement, is the $\operatorname{Sr}$ I at $4607 \AA$. For this reason, this line is highly relevant for this thesis. The potential of this line to determine the turbulent magnetic field strength was first demonstrated by Stenflo (1982) and observations of this line have been first interpreted in terms of the turbulent magnetic field by Faurobert-Scholl (1993).

4 Note that the specific modifications of spectral line scattering polarization are geometry dependent. 
In this thesis we deal with the preliminary step of spatially resolved Hanle measurements: spatially resolved scattering (spectro-)polarimetry in the photosphere. An illustrative example of highly mixed polarity magnetic fields is given in Figure 2.2 (and in the literature for example by Khomenko 2006; Danilovic et al. 2010b; Borrero et al. 2017; Bellot Rubio \& Orozco Suárez 2019), where the line-of-sight inferred magnetic field $B_{l o s}$ from Zeeman measurements (panel b) is compared to a highly resolved (panel c) and spatially degraded (panel d) vertical magnetic field component $B_{z}$ taken from a realistic magneto-hydrodynamic simulation of the solar photosphere observed at disk center. Much of the unsigned magnetic flux (panel c) is undetected when it is observed with a finite, albeit high, spatial resolution, see panel d). The lack of observations to constrain the magnetic topology and energy density on the smallest scales is a prime reason for our lack of understanding of the connection between the small-scale and the large-scale magnetic fields driving solar activity, as well as the Sun's energy balance in general (Stenflo \& Keller 1997; Sánchez Almeida 2005; Trujillo Bueno et al. 2004; Solanki et al. 2006).

Based on the paragraphs above, which highlighted the importance of increasing the knowledge about small-scale turbulent magnetic fields in the photosphere and very briefly introduces the observational method to achieve this, one motivation for this thesis is therefore to pave the way to spatially resolved Hanle observations.

After the discussion of some basic properties of the small-scale turbulent magnetic field we reach the really exciting question: Where does the small-scale turbulent magnetic field component on the solar surface originate, and how does it form?

In contrast to magnetically more active regions, the quiet Sun IN fields show (so far) no evidence for a solar cycle dependence (Kleint et al. 2010; Buehler et al. 2013; Lites et al. 2014). Therefore, it has been suggested that a small-scale turbulent dynamo, independent of the large-scale dynamo responsible for the prominent solar cycle, is operating in and below the photosphere (Petrovay \& Szakály 1993; Cattaneo 1999; Vögler \& Schüssler 2007). ${ }^{5}$ A dynamo process is converting kinetic energy of a plasma to magnetic energy. Most interestingly, Vögler \& Schüssler (2007) showed that with a realistic solar setup in a magnetic-hydrodynamic simulation incorporating a small-scale turbulent dynamo most of the magnetic energy may stay in the photosphere.

Observations show evidence (e.g., Kleint et al. 2011), that the small-scale magnetic field permeating the quiet photosphere interacts strongly with the turbulent convection and therefore may be approximated to be turbulent in the photosphere. The temporal evolution of small-scale magnetic fields probably evolves on time scales of granular lifetimes or shorter (Lites et al. 1996; de Wijn et al. 2009). But the question about an operating dynamo and the energetic coupling mechanisms to other layers of the solar atmosphere is not yet settled. To delve into the details of dynamo theory would be beyond the scope of this thesis. But it is worth to point out that, since the dynamo is a dynamic process, investigating small-scale turbulent magnetic fields temporally in the photosphere would provide an important constraint on solar dynamo theory.

Therefore, an additional motivation of this thesis is that temporal Hanle measurements are needed to track the dynamic development of the small-scale turbulent magnetic field

\footnotetext{
5 One fundamental difference between a small-scale turbulent dynamo and a large-scale dynamo is the generation of a mean magnetic field: in small-scale dynamos, no mean field is generated (Petrovay 2001), which is in agreement with what is observed in the quiet Sun IN magnetic fields.
} 

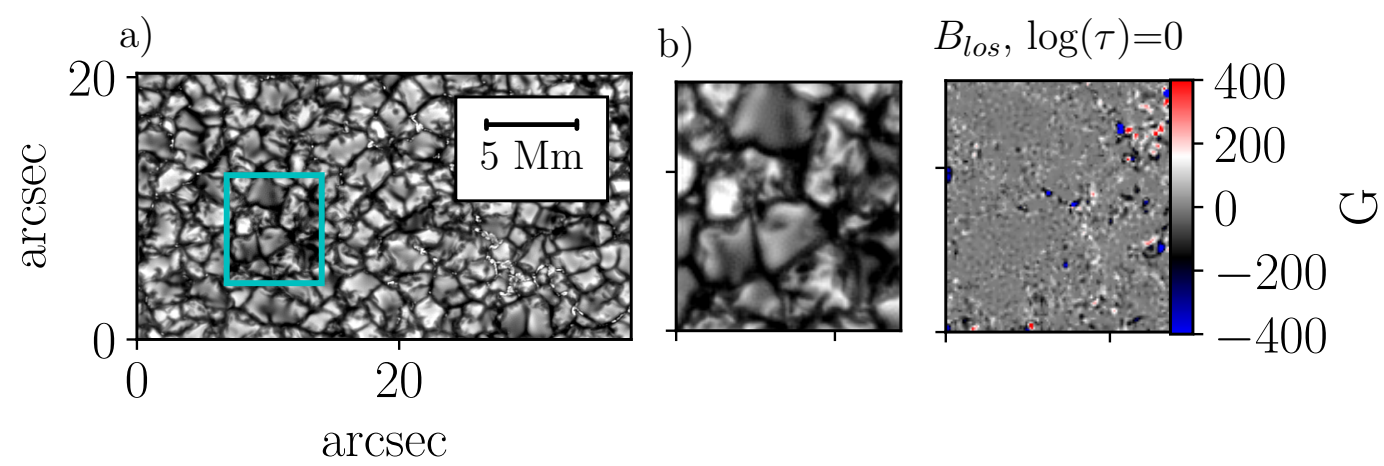

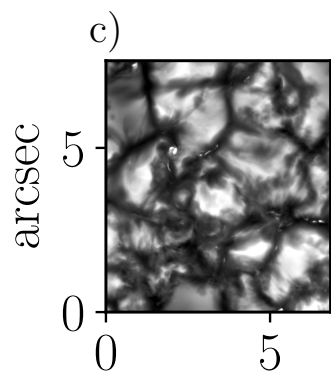

$\operatorname{arcsec}$

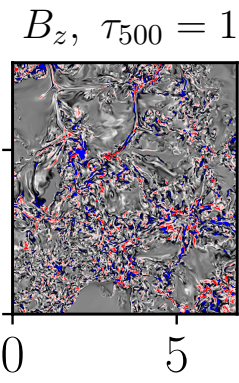

$\operatorname{arcsec}$

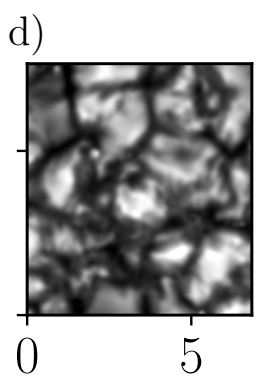

$\operatorname{arcsec}$

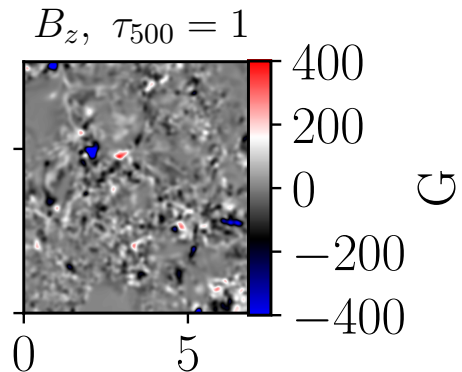

$\operatorname{arcsec}$

Figure 2.2: Quiet Sun continuum intensity images and magnetic field maps normal to the solar surface in observations (top panels) and simulations (bottom panels) at solar disk center at optical depth $(\tau)$ unity. The intensity images are normalized arbitrarily, the magnetic field strength is indicated by the color bar. a) Broadband image of quiet Sun granulation at $630 \mathrm{~nm}$. Observed at the Swedish Solar Telescope (SST), La Palma, with the Microlens Hyperspecrtal imager and context imagers. Data courtesy: Michiel van Noort. b) Sub-field within a), showing details of the granulation and the inferred line-of-sight magnetic field. The data is not corrected for stray-light. The inversion (inference of physical properties) was done with SPINOR (Frutiger et al. 2000) based on STOPRO routines by Solanki (1987). c) High-resolution $(8 \mathrm{~km})$ magneto-hydrodynamic local dynamo simulation (same size as the observation in b) of the photosphere with the MURaM code (Vögler et al. 2005). Data courtesy: Damien Przybylski. d) Same as c), but spatially degraded to the SST sampling. Not only the very detailed structure of the magnetic field is lost during degradation (with very minor effects for the intensity image), but also the amplitude of the vertical magnetic field component is decreased significantly.

together with the plasma dynamics, to challenge local dynamo simulations and to gain insight to the coupling between the solar small-scale and large-scale magnetism, which has implications for other astrophysical problems.

To use the Hanle effect from observations to its full potential in small-scale magnetic field diagnostics, there are still some challenges to overcome. One of the main challenges of the Hanle effect diagnostics is that the amplitude of a given scattering polarization signal depends on several factors, not only the magnetic field. Chief among them being the symmetry property of the pumping radiation field. Such symmetry properties, on the other hand, strongly depend on the thermal and dynamic structure of the solar atmosphere, and they are very sensitive to the presence of horizontal inhomogeneities in the solar 
atmospheric plasma. As this kind of information is not known a priori, it is necessary to develop suitable techniques to disentangle the impact of the magnetic field from that of other symmetry-breaking causes, in order to interpret scattering polarization amplitudes in spectral lines correctly. In the next section we give a brief background on polarization and how it is generated and modified in the solar atmosphere, focusing mainly on the Sr I line. 


\subsection{Light-matter interaction in the solar atmosphere}

The surface of the Sun is constantly radiating, and the properties of the emerging light are coupled to the conditions under which the light is generated. As the Sun is basically a thermal radiator, what we observe is incoherent light. The observables of incoherent light are the energy of the photons (wavelength spectrum), the polarization and the flux (number of photons per unit time and area).

The solar spectrum can be divided into continuum and spectral lines. The latter is the result of transitions in atoms and molecules. Spectral lines in the solar spectrum occur either from emission or absorption of photons.

Polarized radiation is produced every time the interaction of photons with atoms or molecules is affected by symmetry-breaking processes, like the magnetic field or asymmetric (de-)excitation. The spectral line studied in this thesis is $\mathrm{Sr}$ I located at $4607.3 \AA$. It is an absorption line formed in the photosphere. The subject of this chapter is the discussion of how the magnetic field and radiation in the solar atmosphere influences the formation and therefore the polarization of Sr I. Along the way it will be revealed why Sr I is particularly interesting within the numerous spectral lines existing in the solar spectrum. Most of the equations in this chapter are taken from the book of Landi Degl'Innocenti \& Landolfi (2004).

\subsubsection{Polarization: Stokes formalism}

This is a very brief introduction to polarization of light, there are many textbooks describing polarimetry to an excellent detail, e.g. Born \& Wolf (1970).

Monochromatic light is mathematically described as an electromagnetic wave. The wave is fully characterized by wavelength and direction, as well as the phase and amplitudes of the electric field vector with respect to a defined coordinate reference system. In vacuum, the electromagnetic wave is transversal. Therefore, to fully characterize the polarization of light, only three components are needed: the two amplitudes of the electric field vector orthogonal to the propagation direction and the phase relation between them. This complete polarized state of light is described by the Jones formalism (Born \& Wolf 1970).

To mathematically describe quasi-monochromatic, ${ }^{6}$ partially polarized, incoherent radiation, as is the case for solar radiation if measured with narrow spectral filters, the Stokes formalism (Stokes 1852) is used. Light in the Stokes formalism is represented by the Stokes vector $\boldsymbol{I}=(I, Q, U, V)^{\mathrm{T}} .^{7}$ The components of the Stokes vector are in measurable units of (specific) intensity and denote the total intensity $I$, the linear polarization $Q$ and $U$ along two axes with $45^{\circ}$ separation, and circular polarization $V$. For a physically meaningful Stokes vector the relation $I^{2} \geq Q^{2}+U^{2}+V^{2}$ applies. The total degree of polarization $p$ is given by $p=\sqrt{Q^{2}+U^{2}+V^{2}} / I$.

Manipulation and modifications of the Stokes parameters via passive optical elements, like polarizers or retarders, are described by Mueller matrices $\boldsymbol{M}$. The $4 \times 4$ matrices linearly transform one Stokes vector $\boldsymbol{I}$ into a new one $\boldsymbol{I}_{\text {new }}$ :

$$
I_{\text {new }}=M I .
$$

\footnotetext{
6 The spectral frequency of the considered wave is much greater than the spectral bandwidth.

7 Where ${ }^{\mathrm{T}}$ stands for the transpose.
} 
The Mueller matrix includes magneto-optical, anisotropic, scattering, transmission and reflection effects on polarized radiation.

\subsubsection{The radiative transfer equation}

The quantitative description of how unpolarized light is modified while traveling through the solar atmosphere is given by the radiative transfer equation (RTE, extensively described in Chandrasekhar 1960; Mihalas 1978; Hubeny \& Mihalas 2015). More generally, the change of a Stokes vector, $\mathrm{d} \boldsymbol{I}$, along a path length, $\mathrm{d} s$, for a given wavelength in an anisotropic and inhomogeneous medium (in the limit of linear optics and neglecting time dependence) is (Landi Degl'Innocenti \& Landolfi 2004):

$$
\frac{\mathrm{d} \boldsymbol{I}}{\mathrm{d} s}=-\boldsymbol{K} \boldsymbol{I}+\boldsymbol{\epsilon} .
$$

The change of the Stokes vector is determined by the $4 \times 4$ propagation matrix $K$ (removing and redistributing energy) and the emission vector $\boldsymbol{\epsilon}$ (adding energy). The elements of $\boldsymbol{K}$ reflect the direction dependent properties of the medium and are not independent of each other (del Toro Iniesta 2004; Landi Degl'Innocenti \& Landolfi 2004). Altogether there are seven independent matrix elements $K_{i j}$, which account for absorption and magneto-optical effects. To be more specific, there are three constituents to the propagation matrix: absorption $\left(\eta_{I}\right)$, polarization dependent absorption or dichroism $\left(\eta_{I_{i}}\right)$ and dispersion $\left(\rho_{Q, U, V}\right)$ :

$$
\boldsymbol{K}=\left(\begin{array}{cccc}
\eta_{I} & \eta_{Q} & \eta_{U} & \eta_{V} \\
\eta_{Q} & \eta_{I} & \rho_{V} & -\rho_{U} \\
\eta_{U} & -\rho_{V} & \eta_{I} & \rho_{Q} \\
\eta_{V} & \rho_{U} & -\rho_{Q} & \eta_{I}
\end{array}\right)
$$

The specific expressions for $K_{i j}$ can be derived classically or quantum-mechanically (Landi Degl'Innocenti \& Landolfi 2004). One of the remarkable properties of $\boldsymbol{K}$ is that it can not decrease the degree of polarization (del Toro Iniesta \& Collados 2000; Landi Degl'Innocenti \& Landolfi 2004). ${ }^{8}$ In order to fully account even for a decrease or increase of the polarization degree (in a way to make the transfer equation inhomogeneous), it is necessary to introduce a meaningful Stokes-like vector $\boldsymbol{\epsilon}$ for emission processes.

In a spectral line, the emissivities, $\epsilon_{I_{i}}$, are determined by the population of the upper level of the transition, while the $\eta_{I_{i}}$ and $\rho_{Q, U, V}$ coefficients are determined by the population of the lower level. In a state called local thermodynamic equilibrium the populations of both levels are dictated by Boltzmann statistics and polarization is determined by the magnetic field alone. In a scattering medium, the excitation state of the levels is the result of all collisional and radiative processes contributing to their population and relaxation. The problem of solving the RTE becomes non-linear (the absorption and emission coefficients depend on the radiation field itself, see also Sect. 2.3.2) and non-local (Hubeny \& Mihalas 2015). Moreover, the atomic levels may polarize, i.e., the sub-levels are subject to coherences and population imbalances (Landi Degl'Innocenti \& Landolfi 2004).

The most general description of the excitation state of a polarized atom is through its density matrix, further discussed in the next sections.

\footnotetext{
${ }^{8}$ It has been shown that a non-depolarizing medium is described by a symmetric matrix (and all diagonal elements are the same) like $\boldsymbol{K}$ (del Toro Iniesta \& Collados 2000).
} 


\subsubsection{Absorption, emission and the atomic density operator}

Considering an ensemble of strontium ( $\mathrm{Sr}$ ) atoms in an element volume of plasma belonging to the solar atmosphere. These atoms interact with their environment. The environment consists generally of photons, other atoms or ions, and magnetic fields $(B)$. The most important properties of interest of the $\mathrm{Sr}$ atoms are their emissivity and opacity (absorptivity). Both are significantly related to internal characteristics of the atom and external influences.

Generally, the emissivity and opacity are the sum of the spectral line and continuum emissivities and opacities. The contribution for the emissivity and opacity of the Sr I spectral line is the result of atomic resonance transitions, i.e. the $\mathrm{Sr}$ atom changes its energy state. For strong spectral lines, such as Sr I, the emissivities and opacities from the continuum are negligible. Thus, for simplicity, we neglect the continuum contribution to the opacity and emissivity.

The emissivity in the Sr I line comes from a bound-bound transition, this means that an excited atom is releasing energy by changing its electronic configuration from a higher energy state to a lower one (see transition for $B=0 \mathrm{G}$ in Figure 2.3).

Most remarkably, this transition is so interesting for solar physics due to the fact that the upper energy level of the transition has a total angular momentum of $J_{u}=1$ and the lower energy level is the ground state with $J_{\ell}=0$, i.e., unpolarized because of the absence of sub-levels, which results in the maximum achievable polarizability. That is, under appropriate illumination conditions, this transition may polarize the most, see also Sect. 2.3.2. Morevover, Sr I has a Landé factor of $1 .^{9}$ The transition is therefore an excellent text-book example of a two-level atom and can be easily described as such.

Before photons can be emitted the Sr atoms have to be excited - either by absorption or by collisions. We are disentangling the emission and absorption process by assuming complete frequency redistribution. In short, this means that a photon absorbed is not coupled to the photon emitted, a situation ensured for example by frequent collisions or in the case of an incoming radiation field which is spectrally flat, see Sect. 2.3.2.

The emission rate of energy $\epsilon$ (emissivity with units of time, solid angle, volume and wavelength) of a two-level transition with an unpolarized upper level is given by

$$
\epsilon=\frac{h c}{4 \pi \lambda} N n_{u} A_{u \ell} \phi\left(\lambda-\lambda_{0}\right)=\epsilon_{0} \phi\left(\lambda-\lambda_{0}\right),
$$

where $\lambda$ is the wavelength, $\lambda_{0}$ is the wavelength of the emitted photon, $h$ the Planck constant, $c$ the speed of light, $N$ the total number of atoms, given by the solar abundance of $\mathrm{Sr}, n_{u}$ the relative number of atoms in the upper state, $A_{u \ell}$ the Einstein coefficient of spontaneous emission and $\phi\left(\lambda-\lambda_{0}\right)$ the spectral line profile. See Appendix B for a definition of $\phi\left(\lambda-\lambda_{0}\right)$. The spectral line profile takes into account the natural line width given by the lifetime and the dominating Doppler broadening $\Delta \lambda_{D}$ by the turbulent motions of the plasma. A similar expression exists for the opacity, where we neglect stimulated emission - valid for $n_{\ell} \gg n_{u}$, which holds for the blue region of the solar spectrum:

$$
\eta=\frac{h c}{4 \pi \lambda} N n_{l} B_{\ell u} \phi\left(\lambda-\lambda_{0}\right)=\eta_{0} \phi\left(\lambda-\lambda_{0}\right),
$$

\footnotetext{
9 The Landé factor is a quantum-mechanical correction to the classical description, taking into account the effect of the spin of the electron.
} 


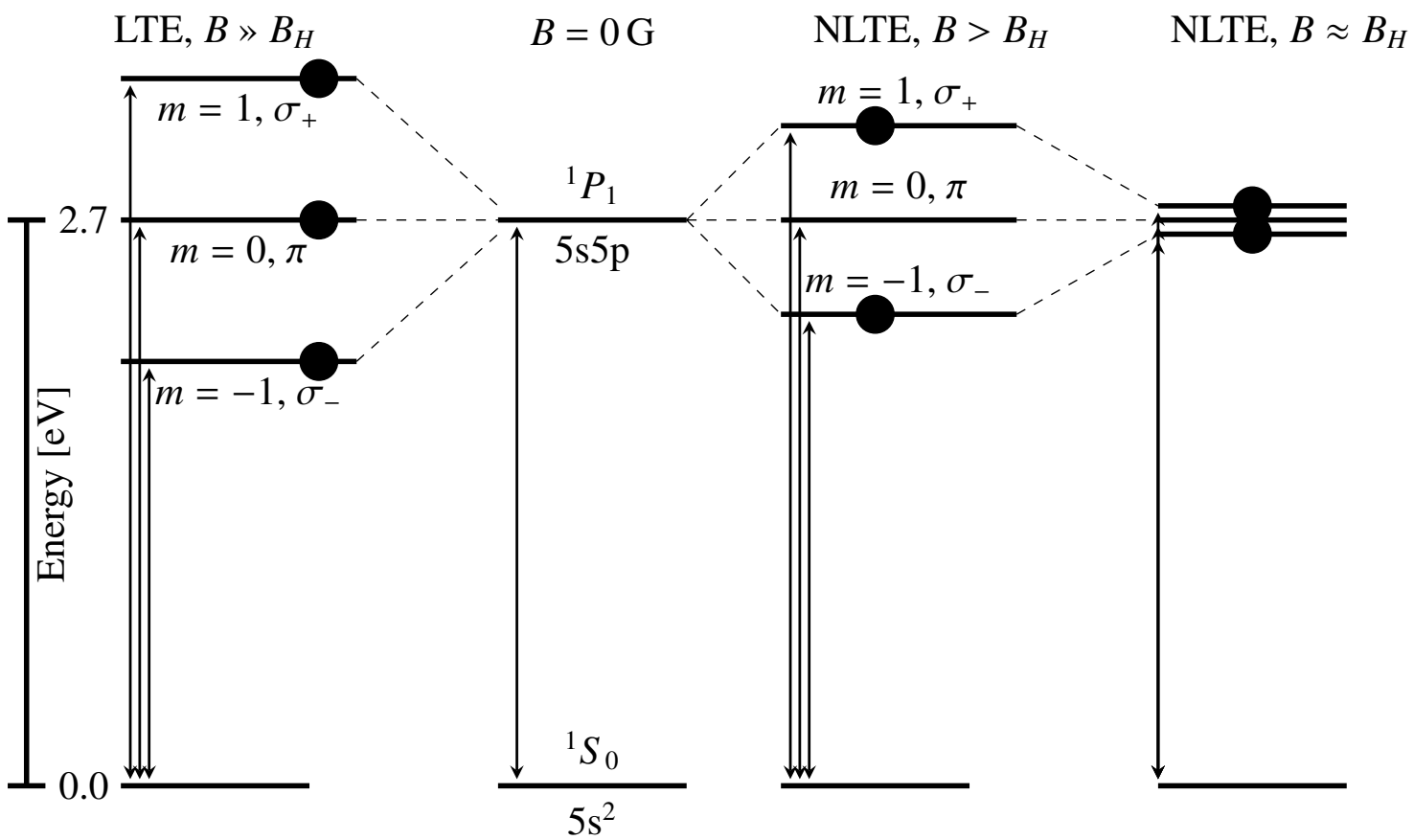

Figure 2.3: Grotrian diagrams for the Sr I transition. In the absence of magnetic fields, $B=0 \mathrm{G}$, Sr I can be treated as a simple two-level atom with the upper level characterized by total angular momentum $J_{u}=1$ and the lower level by $J_{\ell}=0$. Depending on the strength of an external magnetic field, the upper level is sometimes more and sometimes less split into sub-levels denoted by $m$, see left most transition and the two rightmost cases, respectively. The magnetic field strength $B_{H}$ corresponds to a splitting which is on the order of the natural line width of the transition, see Sect. 2.3.2.3. In the case of atomic level polarization due to anisotropic excitation the sub-levels are not equally populated (NLTE case). If the splitting due to the magnetic field is smaller than the line width, the sub-levels may interfere (rightmost diagram).

where $B_{\ell u}$ is the Einstein coefficient for absorption and $n_{\ell}$ the relative number of atoms in the lower state. Remaining notation as in equation (2.4). Note that the unit of the absorption coefficient is given per length and wavelength.

Quantum-mechanically, the $n_{u}$ and $n_{\ell}$ are replaced and generalized by the atomic density matrix $\rho\left(m, m^{\prime}\right)$. The atomic density matrix takes into account the population of the three magnetic sub-levels with quantum number $m$ in the $J_{u}=1$ level and coherences between these sub-levels. The number of magnetic sub-levels $m$ to $J_{u}$ is given by $J_{u}\left(J_{u}+1\right)$ $\left(-J_{u} \leq m \leq J_{u}, m\right.$ integer), with the magnetic field being the quantization axis of the system. With this quantization axis the most convenient basis for the atomic density matrix is $\left|J_{u} m\right\rangle=|m\rangle$, which is the projection of the angular momentum onto the quantization axis. The diagonal elements of the atomic density matrix are interpreted as the population of the magnetic sub-levels (and, therefore, $\left.\sum_{m} \rho(m, m)=n_{u}\right),{ }^{10}$ while off-diagonal elements represent the coherences between the sub-levels.

${ }^{10}$ Note that $\rho$ is Hermitian, thereby the diagonal elements are real and positive. 
In the case of the Sr I line, $J_{u}=1$, and three magnetic sub-states exist. This configuration is called the normal Zeeman-triplet state, see next section for more details. In order to take into account the interferences between the sub-states $m$ and $m^{\prime}$, one has to consider all possible sub-level combinations. The total number of elements of $\rho\left(m, m^{\prime}\right)$ for $J_{u}=1$ is therefore $3 \times 3=9$.

The atomic density matrix elements of the upper level with states $|m\rangle$ is given by

$$
\rho\left(m, m^{\prime}\right)=\left\langle m|\rho| m^{\prime}\right\rangle,
$$

and therefore

$$
n_{u}=\sum_{m=-1}^{1} \rho(m, m)
$$

Analogously, we have a $1 \times 1$ atomic density matrix for the lower level, and since $J_{\ell}=0$ :

$$
\rho\left(J_{\ell}\right)=n_{\ell} .
$$

A generalized version of equation (2.4), derived from quantum electrodynamics in Landi Degl'Innocenti \& Landolfi (2004), takes into account the direction $\Omega$ and polarization of the emitted light (Stokes parameter $I_{i}$, with $i=\{0,1,2,3\}$ ). In the reference frame of a magnetic field (equation (10.39) in Landi Degl'Innocenti \& Landolfi 2004) the emission per Stokes parameter can be expressed as:

$$
\epsilon_{I_{i}}(\lambda, \Omega)=\frac{h c}{4 \pi \lambda} N \sqrt{2 J_{u}+1} A_{u \ell} \times \sum_{k=0}^{2 J_{u}} \sum_{k^{\prime}=0}^{2} \sum_{\substack{q=-k_{M}, k_{M}=\min \left(k, k^{\prime}\right)}}^{k_{M}} \mathcal{T}_{q}^{k^{\prime}}(i, \Omega) \rho_{q}^{k}\left(J_{u}\right) \Phi_{q}^{k k^{\prime}}\left(J_{\ell}, J_{u} ; \lambda\right),
$$

with a spherical representation of the atomic density matrix $\rho_{q}^{k}$, where $k$ is the rank of the tensor $\left(k=0, . ., 2 J_{u}\right)$, while $q$ is the tensor component $(-k \leq q \leq k) .{ }^{11}$ The tensor $\mathcal{T}_{q}^{k}(i, \Omega)$ is taking into account the geometrical configuration of the problem and is tabulated by Landi Degl'Innocenti \& Landolfi (2004), which is reproduced in Appendix A. The profile $\phi(\lambda)$ in equation (2.4) is replaced by the (complex) general profile $\Phi_{q}^{k k^{\prime}}\left(J_{\ell}, J_{u} ; \lambda\right)$, which is just a linear combination of the absorption and dispersion profiles. For the definition of the general line profile, see Appendix B.

From this generalization, it follows that the magnetic sub-levels of the atom are not explicitly written anymore, as the basis is chosen to be spherical and therefore more suitable for a problem which includes the angular momentum of the atomic system. Although the expression (2.9) appears intricate, it highlights the close relationship between polarization and the angular momentum representation of the atomic states. We see later that it is moreover advantageous to use the above expression together with the multipole expansion for the radiation field (Jacobs 1998) to describe scattering polarization.

It is important to note that the spherical representation is essentially a basis change and therefore the elements $\rho_{q}^{k}$ can be related to $\rho\left(m, m^{\prime}\right)$, see Table 2.1. One advantage of the basis change is that the rotational symmetries of the system can be easily represented. To give a few examples: the rank immediately tells how the $\rho_{q}^{k}$ component changes under

\footnotetext{
${ }^{11}$ In the literature, the elements of spherical tensors are usually denoted by $Q$ and $K$. In order to distinguish the notation from the Stokes parameter $Q$, we use $q$ and $k$ instead of $Q$ and $K$, respectively.
} 
Table 2.1: Spherical components $\rho_{q}^{k}$ of the atomic density operator $\rho\left(m, m^{\prime}\right)$ expressed in the standard basis for the atomic $J_{u}=1$ level. Values are adopted from Table 3.6 in Landi Degl'Innocenti \& Landolfi (2004). The elements for negative $q$ can be obtained with $\rho_{-q}^{k}=(-1)^{q}\left(\rho_{q}^{k}\right)^{*}$.

$$
\begin{aligned}
& \rho_{0}^{0}=\frac{1}{\sqrt{3}}[\rho(1,1)+\rho(0,0)+\rho(-1,-1)] \\
& \rho_{0}^{1}=\frac{1}{\sqrt{2}}[\rho(1,1)-\rho(-1,-1)] \\
& \rho_{1}^{1}=-\frac{1}{\sqrt{2}}[\rho(1,0)+\rho(0,-1)] \\
& \rho_{0}^{2}=\frac{1}{\sqrt{6}}[\rho(1,1)-2 \rho(0,0)+\rho(-1,-1)] \\
& \rho_{1}^{2}=-\frac{1}{\sqrt{2}}[\rho(1,0)-\rho(0,-1)] \\
& \rho_{2}^{2}=\rho(1,-1)
\end{aligned}
$$

rotation, i.e. $\rho_{0}^{0}$ is the component which transforms like a scalar under any rotation. Another example is, if the environment is rotationally invariant with respect to the quantization axis. In this case, all components with $q \neq 0$ vanish. The absence of circular polarization in the environment has the consequence that $k=1$ components vanish (Landi Degl'Innocenti \& Landolfi 2004). Further, if the environment is weakly anisotropic, then only two components are left and $\rho_{0}^{0} » \rho_{0}^{2}$, see also Sect. 2.3.2. This clear hierarchy with only two instead of six independent parameters of $\rho$ simplifies a given problem significantly and demonstrates the power of the spherical representation.

The physical interpretation of $\rho$ in the spherical tensor representation is the following (Blum 2012): From Table 2.1 it is easy to see that $\sqrt{3} \rho_{0}^{0}=n_{u}$, therefore this component is connected to the total population of the upper level. If the component $\rho_{q}^{1}$ is non-zero, the atom is said to be oriented. Observationally, atomic orientation is linked to the circular polarization, but is very hard to be distinguished from other effects. We do not discuss further atomic orientation in this thesis, the interested reader is referred to Landi Degl'Innocenti \& Landolfi (2004).

If the component $\rho_{q}^{2}$ is non-zero, we get the case of atomic alignment. We find atomic alignment when the three sub-levels are unequally populated with respect to the $m=0$ state, e.g. $2 \rho(0,0) \neq \rho(1,1)+\rho(-1,-1)$, which is diagrammed in the third example of Figure 2.3. The component $\rho_{2}^{2}$ represents the coherence between the two sub-levels $\rho(1,1)$ and $\rho(-1,-1)$. The observational signature of atomic alignment is linear polarization, which will be covered with more detail in Sect. 2.3.2. Whenever population imbalances and/or coherences among sub-levels exist, that is practically for all $\rho_{q}^{k}$ components except $\rho_{0}^{0}$, we encounter atomic polarization (Trujillo Bueno 2001).

\subsection{Solar generation of spectral line polarization in $\mathrm{Sr} \mathrm{I}$}

Polarized light is always associated with some sort of symmetry breaking processes in the Sun's atmosphere. Symmetry is naturally broken by anisotropic radiation, plasma inhomogeneities and the presence of a magnetic field. Anisotropic radiation causes scattering polarization, while the magnetic field causes energy splittings. Thus, the spectropolarimetric features in spectral lines are sensitive to these processes and reflect the physical conditions under which the spectral lines have been formed. 
We introduce the two main mechanisms producing polarization in spectral lines: the Zeeman effect and scattering. The former is a symmetry break caused by magnetic fields and, as a consequence, spectral line splitting and polarization, while the latter is spectral line polarization as a result of an inhomogeneous (asymmetric) radiation field. Our aim is to present both mechanisms in a coherent framework. Although the formalism is general (Landi Degl'Innocenti \& Landolfi 2004), we particularize the analysis to the Sr I resonance line at $4607 \AA$, which is the main focus of this thesis.

\subsubsection{The Zeeman effect}

Classically, the process of emission can be described by an electron oscillating with frequency $v_{0}$, corresponding to a wavelength $\lambda_{0}=c / v_{0}$, around the nucleus (dipole oscillation, see e.g. Landau \& Lifshitz 2013). For convenience, the oscillation is decomposed into a linear oscillation along some arbitrary direction and two opposing circular oscillators in the orthogonal plane, see Figure 2.4 for the geometry. All of the oscillators in this scenario are assumed to be excited independently and are therefore decoupled. What happens to the emission of $\mathrm{Sr}$ atoms if they are exposed to a homogeneous and static magnetic field $B$ ? Let the magnetic field be aligned along the linear oscillator. The energy of the linear oscillator is unaffected by the magnetic field, the emergent radiation is linearly polarized and is called the $\pi$ component. However, each circular oscillator will precess around the magnetic field vector with the Larmor frequency $v_{L}$, hence their total frequency is shifted (see textbooks by Jackson 1998; Landau \& Lifshitz 2013). The energies of the two circular oscillators are shifted by $\Delta v= \pm v_{L}$ : one oscillator has more energy while the energy of the other oscillator decreased. The Larmor frequency is given by

$$
v_{L}=\frac{e B}{4 \pi m_{e} c}=1.3996 \times 10^{6} B \mathrm{G}^{-1} \mathrm{~s}^{-1},
$$

with $B$ in $\mathrm{G}, v_{L}$ in $\mathrm{s}^{-1}$, and the electron charge and mass $e$ and $m_{e}$, respectively (Landi Degl'Innocenti \& Landolfi 2004).

The emerging higher and lower energy radiation components are called $\sigma_{+}$and $\sigma_{-}$, respectively. The polarization of the $\sigma$ components seen by the observer depends on the line-of-sight relative to the magnetic field vector. There are two special cases: either the line-of-sight is aligned with the magnetic field vector, then the $\sigma$ components are circularly polarized, see also Figure 2.4. In the other case, where the line-of-sight is in the plane of the two circular oscillators, the $\sigma$ components are linearly polarized. The spectrally separated and differently polarized components are associated with the Zeeman effect (Zeeman 1897).

Quantum-mechanically, the energy of the excited Sr atoms is determined by the quantum numbers $J_{u}$ of the total angular momentum and $m$ of the upper level. In the SrI case where $J_{u}=1, m=0, \pm 1$. In the presence of a magnetic field, the sub-levels $m$ are not degenerate anymore and the three transitions to the ground state with $m_{\ell}=0$ result in spectral lines centered at slightly different wavelength positions $\lambda_{m}$, where $\lambda_{0}$ is the center of the profile in the absence of a magnetic field, see leftmost case of Figure 2.3:

$$
\lambda_{m}=\lambda_{0}+m g \frac{e \lambda_{0}^{2} B}{4 \pi m_{e} c^{2}}=\lambda_{0}+m g \lambda_{0}^{2} \frac{v_{L}}{c}=\lambda_{0}+m \Delta \lambda_{L}
$$




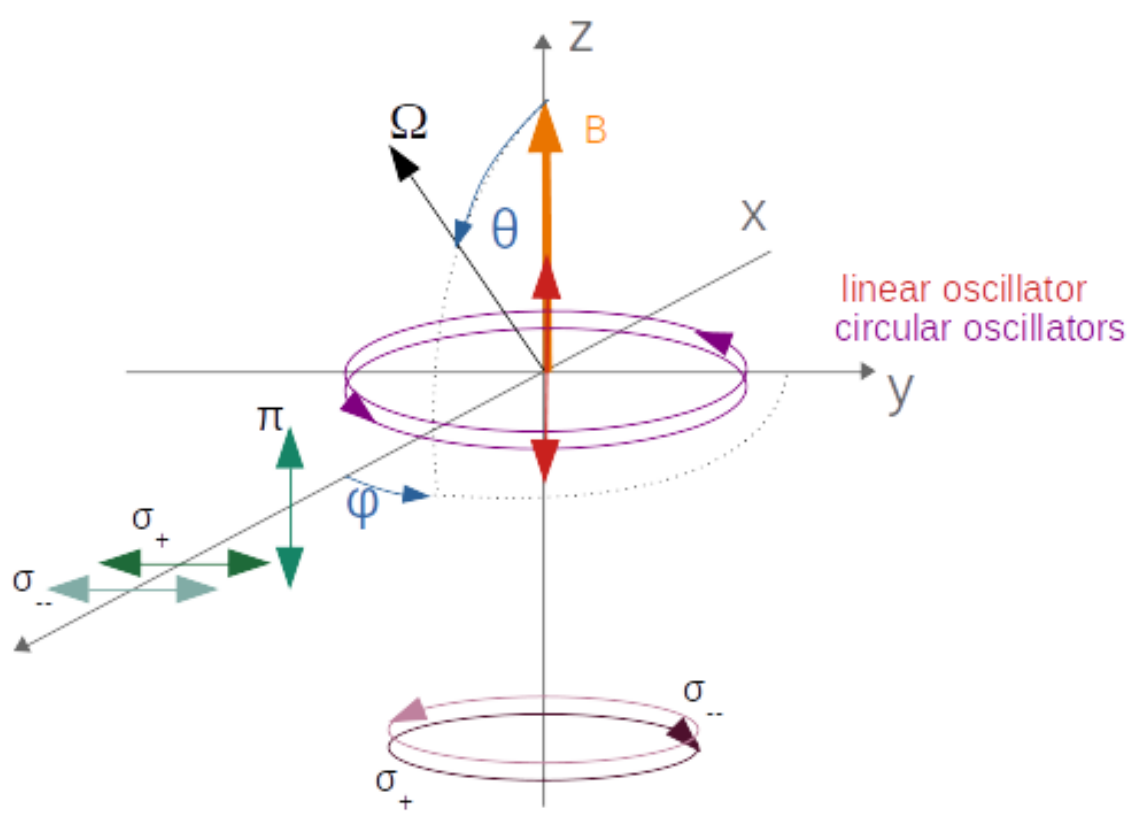

Figure 2.4: Geometry of the emitted radiation in the direction $\Omega$, with angles $\theta$ and $\varphi$ with respect to the magnetic field vector, of a classical oscillator in the presence of a magnetic field with strength $B$. Two special cases, when the line-of-sight is along the direction of the magnetic field (i.e., along the $\pm z$ axis) or perpendicular to it (i.e., in the $x-y$ plane), correspond to the longitudinal (only circular polarization emitted) and transversal (only linear polarization is emitted) Zeeman cases, respectively.

The factor $g$ is the Landé-factor ${ }^{12}$ for the upper level, for Sr $\mathrm{I} g=1$. Note that then equation (2.11) gives the exact result which holds in the classic framework. This case, when there are three Zeeman components and the spin is neglected, is called normal Zeeman effect. This equivalence between the classical and the quantum mechanical models allows that the magnetic sub-levels $m=1$ and $m=-1$ are associated with the $\sigma_{+}$and $\sigma_{-}$, respectively, while the $\pi$ component is the transition from the $m=0$ sub-level to the ground level. One important note: The equations only hold true in the case that the magnetic energy is smaller compared to the coupling of the angular momentum with the spin momentum ( $L-S$-coupling regime).

When the Zeeman effect is present, it is possible to derive the emergent components of the Stokes vector for Sr I using equation (2.9). The independently excited oscillators in the classical model have a quantum mechanical analogue which is the balanced (and incoherent, see below) population of all three sub-levels, given by isotropic excitation for example. In this case, the only component of the atomic density matrix is $\rho_{0}^{0}$. Therefore, the emissivity depends only on the total population of the upper level and the splitting of the spectral line components. Consequentially, there are only three components of the

\footnotetext{
${ }^{12}$ In $L-S$ coupling, the Landé-factor (dimensionless) (Landé 1923) is generally given by $g=1+$ $\frac{1}{2} \frac{J(J+1)+S(S+1)-L(L+1)}{J(J+1)}$, with $J=L+S$, where $L$ and $S$ are the orbital and spin quantum numbers, respectively. For $\operatorname{Sr}$ I, $S=0$.
} 
general profile. With $\rho_{0}^{0}=n_{u} / \sqrt{3}$ and $\sqrt{2 J_{u}+1}=\sqrt{3}$, equation (2.9) reduces to

$$
\epsilon_{I_{i}}(\lambda, \Omega)=\sqrt{3} \epsilon_{0} / \sqrt{3}\left[\mathcal{T}_{0}^{0}(i, \Omega) \Phi_{0}^{00}(\lambda)+\mathcal{T}_{0}^{1}(i, \Omega) \Phi_{0}^{01}(\lambda)+\mathcal{T}_{0}^{2}(i, \Omega) \Phi_{0}^{02}(\lambda)\right] .
$$

For $\epsilon_{0}$, see equation (2.4). The values of $\mathcal{T}_{0}^{0,1,2}$ can be found in Table A.1, where also the angles for the geometrical tensor are defined. For the emergent radiation, $\theta$ and $\varphi$ are the angles of the emission direction $\Omega$ with the magnetic field, for the geometry see Figure 2.4. For simplicity, we set $\varphi=0$. Then the emergent Stokes parameters (similar to equations (3.40) in Landi Degl'Innocenti \& Landolfi 2004) are given by:

$$
\begin{aligned}
& \epsilon_{I}(\lambda, \Omega)=\epsilon_{I}(\lambda, \theta)=\frac{\epsilon_{0}}{2}\left(\frac{1+\cos ^{2}(\theta)}{2}\left(\phi^{-1}+\phi^{1}\right)+\phi^{0} \sin ^{2}(\theta)\right) \\
& \epsilon_{Q}(\lambda, \Omega)=\epsilon_{Q}(\lambda, \theta)=\frac{\epsilon_{0}}{2}\left(\phi^{0}-\frac{\phi^{-1}+\phi^{1}}{2}\right) \sin ^{2}(\theta) \\
& \epsilon_{U}(\lambda, \Omega)=\epsilon_{U}(\lambda, \theta)=0 \\
& \epsilon_{V}(\lambda, \Omega)=\epsilon_{V}(\lambda, \theta)=\frac{\epsilon_{0}}{2}\left(\phi^{1}-\phi^{-1}\right) \cos (\theta) .
\end{aligned}
$$

The superscript of the three spectral profiles $\phi^{m}$ denote the corresponding magnetic sublevel $m$ from where the atom is de-excited (see Appendix B for the functional form of $\left.\phi^{m}\right)$. The profiles are centered at $\lambda_{m}$, i.e $\phi^{m}=\phi^{0}\left(\lambda-\lambda_{m}\right)$. As an example, the intensity Stokes parameter $I$ is spectrally split (with either two or three components depending on the geometry) if the energy split by the magnetic field is significantly larger than the spectral line width. A magnetic field (anti-)parallel to the line-of-sight $\left(\theta=0^{\circ}\right.$ and $\left.\theta=180^{\circ}\right)$ generates circular polarization with an antisymmetric spectral shape of the spectral line, the longitudinal Zeeman effect. In the bottom panel of Figure 2.5 we plot examples of the normalized circular polarization $(V / I)$ profiles for a sample of magnetic field strengths. The polarity of the magnetic field with the line-of-sight can be distinguished in the Stokes $V / I$ profiles, as the parallel and anti-parallel profiles have different signs. For example profiles, see equation (2.13a) and Figure 2.5. A magnetic field perpendicular to the line-of-sight (e.g., $\theta=\pi / 2$ ) results in a symmetric spectral shape of the spectral line, which is linearly polarized, the transversal Zeeman effect.

If the magnetic field is weak, which means that the spectral line width $\Delta \lambda_{D}$ is larger than the Zeeman splitting $\Delta \lambda_{B}=\lambda_{m}-\lambda_{0}$, then the ratio between the two is small $(\xi=$ $\left.\Delta \lambda_{B} / \Delta \lambda_{D} \ll 1\right)$. In this weak magnetic field regime the profile is not fully split, but at most broadened. Then the profiles $\epsilon_{I_{i}}$ may be expanded with respect to $\xi$, which is called the weak field approximation (del Toro Iniesta \& Collados 2000; Landi Degl'Innocenti \& Landolfi 2004). The zeroth order is given by the intensity $I$ alone. The spectral symmetry of the linear and circular Zeeman components distinguishes between the nodes (circular: one node, linear: two nodes). Then the linear and circular polarization occur in different orders of the expansion. To first order, Stokes $V$ is proportional to $B \partial I / \partial \lambda$. The linear polarization components are proportional to the second derivative, $B^{2} \partial^{2} I / \partial \lambda^{2}$. This means that, for weak magnetic fields, the longitudinal Zeeman effect is more sensitive to magnetic field strengths than the transversal Zeeman effect.

The mere detection of spectral line polarization signatures resulting from the Zeeman effect can be directly explained by the magnetic field presence. Therefore, because of this relatively simple interpretation of polarized spectral line profiles, the Zeeman effect is a 


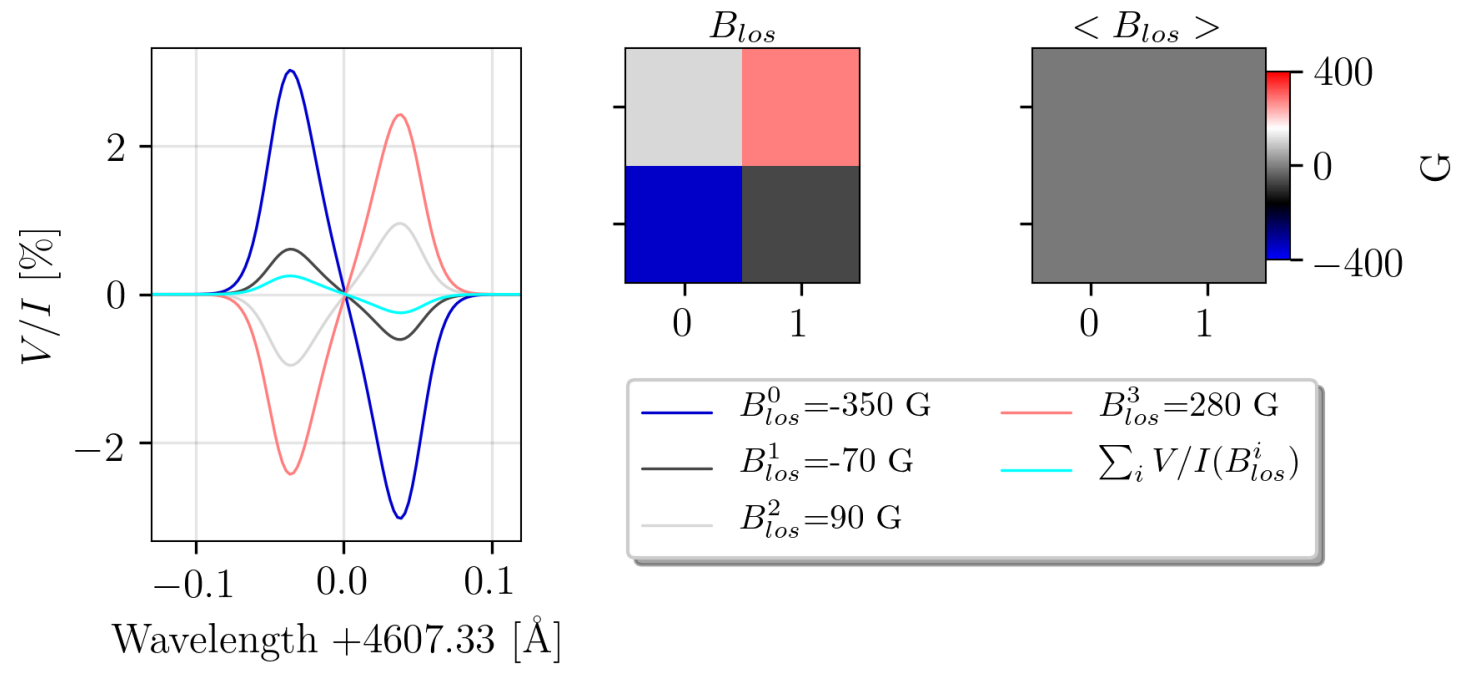

Figure 2.5: Mixed polarity magnetic fields in the line-of-sight and corresponding Stokes $V / I$ profiles calculated with the weak field approximation for Sr I. If the region is observed with high spatial resolution (center panel), the Stokes $V / I$ profiles are easily distinguishable (left panel, the line colour corresponds to the line-of-sight magnetic field in the center panel). Because of the sign reversal of the profiles, observing at lower spatial resolution (right panel) the (net) amplitude of the spatially integrated Stokes V/I profiles (cyan line in left panel) is strongly reduced.

widely used tool to diagnose astrophysical magnetic fields (e.g., Donati et al. 1997; Stenflo \& Keller 1996; Trujillo Bueno 2003; Solanki et al. 2006; Casini et al. 2008; Bellot Rubio \& Orozco Suárez 2019; Oklopcic et al. 2019). The $V$ profile is proportional to $\cos (\theta)$ and therefore especially sensitive to the polarity of the magnetic field. The implication for diagnostics is that the polarity of the observed magnetic field is encoded in the circular polarization profile. However, in the case of highly mixed polarity fields within a resolution element, the spectral components cancel. This important limitation of Zeeman diagnostics implies that inferences of the magnetic field are upper limit estimates and depend on the spatial resolution (e.g., Shchukina \& Trujillo Bueno 2013, and references therein). In Figure 2.5 we show profiles of Stokes $V / I$ in the weak field approximation for $\mathrm{Sr}$ I in a region which is dominated by mixed polarity magnetic fields in the line-of-sight. A poorly resolved region which is dominated by mixed polarity magnetic fields results in a low Stokes $V / I$ profile amplitude (see cyan profile, which is the average of the other profiles in Figure 2.5). If only a fraction $f_{B}$ of the pixel is filled with a magnetic field of strength $B$ (i.e., $B=0 \mathrm{G}$ for a fraction of $\left(1-f_{B}\right)$ of the pixel) then the circular polarization observed in this pixel is given by (e.g., Bellot Rubio \& Orozco Suárez 2019)

$$
V=0 \cdot \frac{\partial I}{\partial \lambda} \cdot\left(1-f_{B}\right)+B \frac{\partial I}{\partial \lambda} \cdot f_{B}=B \cdot f_{B} \cdot \frac{\partial I}{\partial \lambda} .
$$

The factor $B \cdot f_{B}=<B>$ (where $<B>$ is the spatial mean) is sometimes referred to as the magnetic flux density (e.g., Keller et al. 1994a). Hence, to estimate $B, f_{B}$ has to be assumed. If the magnetic field is highly tangled (even in the case of $f_{B}=1$ ) the cancellation in some cases may be very efficient and the pixel would be filled with zero circular polarization caused by a zero magnetic flux density (see for example two top panels of Figure 2.5). 
Therefore, the longitudinal Zeeman effect is blind to magnetic field topologies with zero net magnetic flux densities along the line-of-sight, which is the case if the pixel is filled with highly mixed polarity fields of equal strengths (Stenflo 1988; Solanki et al. 2006). These kinds of magnetic fields would be "hidden" (Stenflo 1988). One implication is that the magnetic energy, which is proportional to $B^{2}$, associated with a spatially poorly resolved mixed polarity region is much lower than if it is highly resolved, see Figure 2.2. This limitation does also affect measurements for spatially unresolved stellar spectra (e.g., Reiners 2012).

To determine the field strength of a small-scale turbulent component of the magnetic field with Zeeman diagnostics the so-called line-ratio technique has been proposed (Unno 1959; Stenflo 1973; Keller et al. 1994b). The line-ratio of two observed similar spectral lines with different Landé factors reveals differences in the Doppler widths. However, this technique still requires a small net Zeeman signal and two lines which otherwise formed under almost identical conditions.

To summarize, the Zeeman effect is of limited relevance for the diagnostics of complex magnetic field geometries at small spatial scales. Moreover, these are very interesting magnetic fields, in particular in intergranules. An alternative diagnostic tool is scattering polarization modified by the magnetic field, which is sensitive to unresolved magnetic fields. This is addressed in the next sections.

Note that in the solar photosphere the Zeeman effect is usually observed in absorption, not emission lines. This results from the temperature gradient in the solar atmosphere. The plasma is dense enough to ensure that the collisional rates are in equilibrium with the radiative rates. This ensures an equilibrium state of the local plasma with its surrounding or, in short, a single temperature is used to describe radiation and the plasma. This state is defined as local thermodynamic equilibrium (LTE), which is usually a good approximation for the solar photosphere. The emission in LTE is only given by the Kirchhoff-Planck function $B_{\lambda}(T)$ (Stix 2004), which has a temperature dependence. Since the temperature decreases towards the temperature minimum, the emission is decreased in upper layers of the lower solar atmosphere. As the opacity in spectral lines is greater than in the continuum, the spectral lines in the photosphere are formed higher than the continuum. Higher layers emit less radiation, what is observed therefore are absorption spectral lines. However, the expressions for the emission shown above are proportional to the very familiar absorption coefficients usually used in the RTE for the Zeeman effect (del Toro Iniesta \& Collados 2000; Landi Degl'Innocenti \& Landolfi 2004). Therefore, despite some minor details such as the sign of the Stokes $V$ profile, the physics is well described by equations (2.13a).

\subsubsection{Scattering polarization}

Scattering is also capable of generating polarization in spectral lines in the solar atmosphere. One necessary condition for scattering polarization is that the scattering particles are anisotropically illuminated. Scattering in spectral lines may generate linear polarization. The diagnostic potential of spectral line scattering polarization lies in its sensitivity to the presence of magnetic fields via the Hanle effect (see Sect. 2.3.2.3). For the classical description of scattering, see Figure 2.6.

How can we formally describe the simplest case of scattering, where we have to take into account the incident (and in the first approximation unpolarized) radiation? 
Scattering polarization can be described by a series of absorption and emission processes. Before we directly use the general equation (2.9) for the emission, let us first take a simple example for an emission process, which depends on an arbitrary incident radiation field.

The incident radiation (from the direction $\Omega^{\prime}=-\Omega$ in Figure 2.4) on a scatterer, placed in the center of the coordinate system in Figure 2.4, can be described by $I^{i n c}\left(\lambda, \theta^{\prime}, \varphi^{\prime}\right)$, considering the direction dependence in the polar and azimuth angles, $\theta^{\prime}$ and $\varphi^{\prime}$, respectively. We prime the angles of incidence to distinguish from the angles of the emitted radiation. Assuming that all the absorbed radiation is going to be isotropically (and coherently ${ }^{13}$ ) scattered, respectively emitted,${ }^{14}$ the emission coefficient may be written as:

$$
\epsilon(\lambda)=\int I^{i n c}\left(\lambda, \theta^{\prime}, \varphi^{\prime}\right) \eta(\lambda) \cos \left(\theta^{\prime}\right) \mathrm{d} \theta^{\prime} \mathrm{d} \varphi^{\prime}=\int I^{i n c}\left(\lambda, \Omega^{\prime}\right) \eta(\lambda) \mathrm{d} \Omega^{\prime},
$$

where $\eta$ is the absorption coefficient defined in equation (2.5). This simple expression already demonstrates the challenges arising when the RTE in equation (2.2) has to be solved and scattering is considered: the radiation itself enters the emission part of the RTE in a nonlinear fashion, which means that the incident radiation has to be known from all directions, and makes the whole problem non-linear. Radiation is usually a non-local quantity, thereby the thermodynamic conditions under which the incident radiation is generated may differ significantly from the local conditions of the region where the radiation is scattered. Besides this non-locality, the scattering portion of the atmosphere deviates from local thermodynamic equilibrium, i.e. the emitted radiation cannot be described by the Planck function. These conditions are defined as non-local thermodynamic equilibrium (NLTE). Sophisticated numerical schemes need to be implemented to solve the RTE in NLTE conditions.

Assuming that the absorption is angle independent, equation (2.15) becomes:

$$
\epsilon(\lambda) / \eta(\lambda)=\int I^{i n c}\left(\lambda, \Omega^{\prime}\right) \mathrm{d} \Omega^{\prime}=4 \pi J(\lambda) .
$$

It is easy to verify that the integral when divided by $4 \pi$ is the mean intensity $J(\lambda)$.

It come in handy if we express the incident radiation in terms of its spherical components, like we did for the atomic density matrix. The components of the radiation field tensor are denoted by $J_{q}^{k}$ with $0 \leq k \leq 2$ and $-k \leq q \leq k$. The complex components $J_{q}^{k}$ are defined using the Stokes parameters $I_{i}$ by: ${ }^{15}$

$$
J_{q}^{k}(\lambda)=\int \frac{\mathrm{d} \Omega^{\prime}}{4 \pi} \sum_{i=0}^{3} \mathcal{T}_{q}^{k}\left(i, \Omega^{\prime}\right) I_{i}\left(\lambda, \Omega^{\prime}\right) .
$$

The mean intensity is the radiation tensor component $J_{0}^{0}(\lambda)=J(\lambda)$.

In the case of unpolarized incident radiation (i.e., $\left.\boldsymbol{I}^{\text {inc }}=\left(I^{i n c}, 0,0,0\right)^{\mathrm{T}}\right)$, which is a good approximation for the quiet solar photosphere, the components with $k=1$ vanish.

\footnotetext{
${ }^{13}$ This means that the energy of the absorbed and emitted photon by an isolated atom are the same, which means that a fixed phase relation between the two photons can be defined.

${ }^{14}$ Note that in equation (2.4) the excitation process is not specified.

${ }^{15}$ Equation (5.157) in Landi Degl'Innocenti \& Landolfi (2004), with $J_{-q}^{k}=(-1)^{q}\left(J_{q}^{k}\right)^{*}$, where “*” is the complex conjugate.
} 
For $k=2, J_{q}^{k}$ can be expressed in terms of the complex-valued spherical harmonics $Y_{\ell}^{m}(\Omega)=Y_{q}^{k}(\Omega)$ (chapter 12.4 in Landi Degl'Innocenti \& Landolfi 2004):

$$
J_{q}^{2}=\sqrt{\frac{2 \pi}{5}} \int \frac{\mathrm{d} \Omega^{\prime}}{4 \pi} Y_{q}^{2}\left(\Omega^{\prime}\right) I\left(\Omega^{\prime}\right),
$$

where it is assumed further that the incident radiation is spectrally flat, i.e. $I^{\text {inc }}\left(\lambda, \Omega^{\prime}\right)=$ $I^{i n c}\left(\Omega^{\prime}\right)$. Thus, the wavelength dependence is dropped. ${ }^{16}$ Atoms in the solar atmosphere are not isolated, but are constantly exposed to collisions, magnetic fields, etc. Thereby, in the scattering process, the phase relation between the incoming and scattered photon is destroyed. The energies of the absorbed and emitted photons are not correlated any longer, and the specific energies of the incoming and the emitted photons are independent of each other. Therefore, the incoming radiation spectrum can be assumed to be flat. In Sect. 2.2.3 we referred to this as complete frequency redistribution (CRD). Note that this is a strong assumption. A more accurate (but also much more complicated) way to describe the scattering process is to assume partial frequency redistribution (PRD), where the energy and the direction of the emitted photon is correlated with the incoming photon. The impact of PRD effects has been vastly demonstrated, e.g. by Milkey \& Mihalas (1973), Faurobert et al. (1987), Frisch (1996), Sampoorna et al. (2010) and Belluzzi \& Trujillo Bueno (2012), but mainly for strong chromospheric lines. However, Alsina Ballester et al. (2017) showed that the CRD limit is a very good approximation for modeling the intensity and polarization of the emitted radiation in the case of the Sr I line.

Moreover, in the case of resonance polarization, the atomic density matrix elements $\rho_{q}^{k}$ can be obtained in terms of the incoming radiation, as the population of the upper level is determined by the radiation field. If we choose the quantization axis along the scattered radiation, equation (10.13) in Landi Degl'Innocenti \& Landolfi (2004) describes the connection between the radiation field tensor elements $J_{q}^{k}$ and the atomic density elements $\rho_{q}^{k}$ in the collisionless limit: ${ }^{17}$

$$
\rho_{q}^{k}=\frac{1}{\sqrt{3}} \frac{B_{\ell u}}{A_{u \ell}}(-1)^{q} J_{-q}^{k} \rho_{0}^{0}\left(J_{l}\right)
$$

Note that the $\rho_{q}^{k}$ and $J_{-q}^{k}$ elements with different $k$ and $q$ are decoupled. This conveys that the order of the radiation field translates into the order of the atomic density matrix. As each element of $\rho_{q}^{k}$ relates to the population of the sub-levels of the atom (Trujillo Bueno 2001), anisotropic incident radiation on a scatterer can selectively populate the Zeeman sub-levels.

In principle, the atomic sub-levels can be disregarded when calculating the emission, i.e. the scattered radiation. That is achieved by inserting equation (2.19) into equation (2.9).

\footnotetext{
${ }^{16}$ If the incident radiation is isotropic (e.g., $I^{i n c}\left(\Omega^{\prime}\right)=I^{i n c}$ ), then $J_{q}^{2}=0$, and the classical expression for scattering is recovered (Mihalas 1978).

${ }^{17}$ Note that this is a reasonable approximation for the lower solar atmosphere, where the plasma density and temperature are relatively low. To include collisions (for SrI), the factor $J_{-q}^{k}$ is replaced by $\left(c B_{\lambda}(T) \delta_{k 0} \delta_{q 0}+\right.$ $\left.J_{-q}^{k}\right) /(1+c+\delta$ ) (equation (10.50) in Landi Degl'Innocenti \& Landolfi 2004), where $c$ and $\delta$ represent the ratio of collisional to radiative de-excitation rates of the upper level and the effective number of de-polarizing collisions, respectively.
} 
Furthermore, the assumptions we introduced above (i.e., the incident radiation is unpolarized and spectrally flat) are used. For $\operatorname{Sr}$ I, $J_{u}=1$ and $J_{\ell}=0$, although the slightly more general expressions are kept to make the equations more clear. Further, we use the general spectral line profile in the limit of a zero magnetic field (see Appendix B):

$$
\lim _{v_{L} \rightarrow 0} \Phi_{q}^{k k^{\prime}}\left(J_{\ell}, J_{u} ; \lambda\right)=\delta_{k k^{\prime}} \phi\left(\lambda-\lambda_{0}\right) .
$$

For the emission, we then get:

$$
\epsilon_{I_{i}}(\lambda, \Omega)=\frac{h c}{4 \pi \lambda} N \sqrt{3} A_{u l} \sum_{k q} \mathcal{T}_{q}^{k}(i, \Omega) \frac{1}{\sqrt{3}} \frac{B_{\ell u}}{A_{u \ell}}(-1)^{q} J_{-q}^{k} \rho_{0}^{0}\left(J_{\ell}\right) \phi\left(\lambda-\lambda_{0}\right) .
$$

Identifying $\rho_{0}^{0}\left(J_{\ell}\right)=n_{\ell} / \sqrt{3}$, we simplify equation (2.21) to:

$$
\epsilon_{I_{i}}(\lambda, \Omega)=\frac{\eta(\lambda)}{\sqrt{3}} \sum_{k q} \mathcal{T}_{q}^{k}(i, \Omega)(-1)^{q} J_{-q}^{k},
$$

where $\eta(\lambda)$ is defined in equation (2.5). Note that equation (2.22) is a more generalized version of equation (2.16), considering the polarization, the symmetry of the radiation field and the direction of the scattered radiation. 


\subsubsection{Scattering polarization at the limb: $\mathrm{Sr} \mathrm{I}$ in the second solar spectrum}

In the solar atmosphere, in general the radiation at any point is clearly not isotropic. The anisotropy of the radiation field arises due to outwardly escaping radiation from the solar interior, but also due to horizontal inhomogeneities, i.e due to granules. When observing the solar limb, the former dominates over horizontal fluctuations. Thus, neglecting local horizontal fluctuations of the radiation, the incident radiation field can be described as axially symmetric. Due to limb darkening, the incident radiation dependents on the polar angle $\theta^{\prime}$. Then, besides the mean intensity $J_{0}^{0}$, the only other non-zero component of the radiation tensor, based on the assumptions mentioned above, is $J_{0}^{2}$.

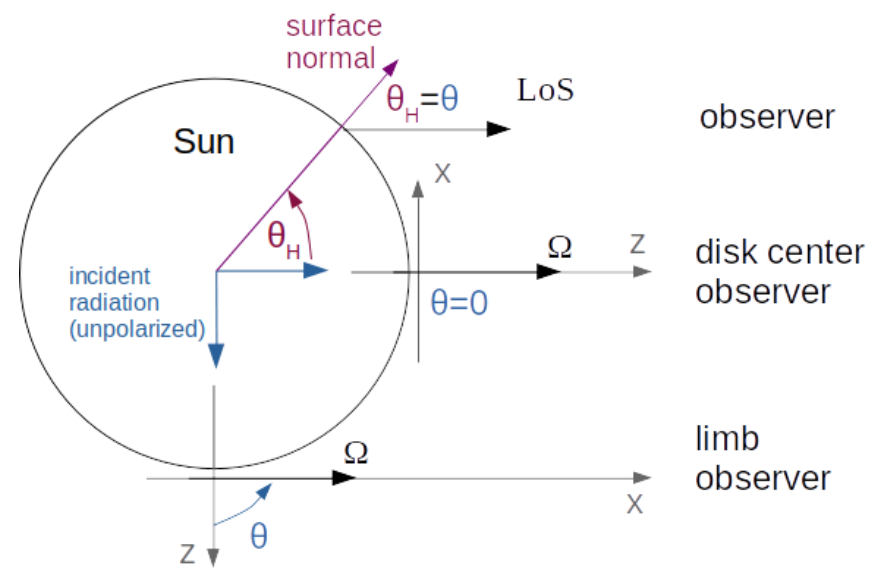

Figure 2.7: Geometry for limb and disk center observations. The quantization axis $z$ is given by the direction of the incident radiation. The direction of the scattered light is $\Omega$. The definition of $\theta$ is consistent with the other figures. Usually, the heliocentric angle $\theta_{\mathrm{H}}$ is given by the surface normal and the line-of-sight $(\operatorname{LoS})$, and is used to define the distance between the limb and disk center. With our definitions, these angles coincide, i.e. $\theta_{\mathrm{H}}=\theta$.

The ratio $\mathcal{A}=J_{0}^{2} / J_{0}^{0}$ is known as the anisotropy factor. To formally derive the formation of linear polarized radiation at the limb from equation (2.22) is beyond the scope of this thesis, but an educational example can be found in Trujillo Bueno (2003b). However, it can be shown that the fractional linear polarization $Q / I$ parallel to the solar limb for Sr I can be written as (Trujillo Bueno 2005, and references therein)

$$
\epsilon_{Q} / \epsilon_{I}=Q / I \approx \frac{3}{2 \sqrt{2}}\left(1-\mu^{2}\right) \mathcal{A},
$$

where $\mu=\cos (\theta)$ is the viewing direction of the observer given by the heliocentric angle $\theta$, see Figure 2.7 for the definition of the heliocentric angle. Equation (2.23) applies in the collisionless and zero magnetic field limit. The linear polarization parallel to the solar limb is therefore proportionally increasing with the anisotropy and also scales with the distance to the solar disk center, with a maximum close to the limb.

Scattering polarization of spectral lines at the limb was discovered by Harvey et al. (1980). The first systematical mapping of spectral line scattering polarization at the limb was done by Stenflo et al. (1983). Since then, there is an increasing interest to routinely 
measure scattering polarization in spectral lines close to the solar limb (Gandorfer 2000, 2002, 2005). The term second solar spectrum has prevailed for the polarized spectrum measured close to the solar limb.

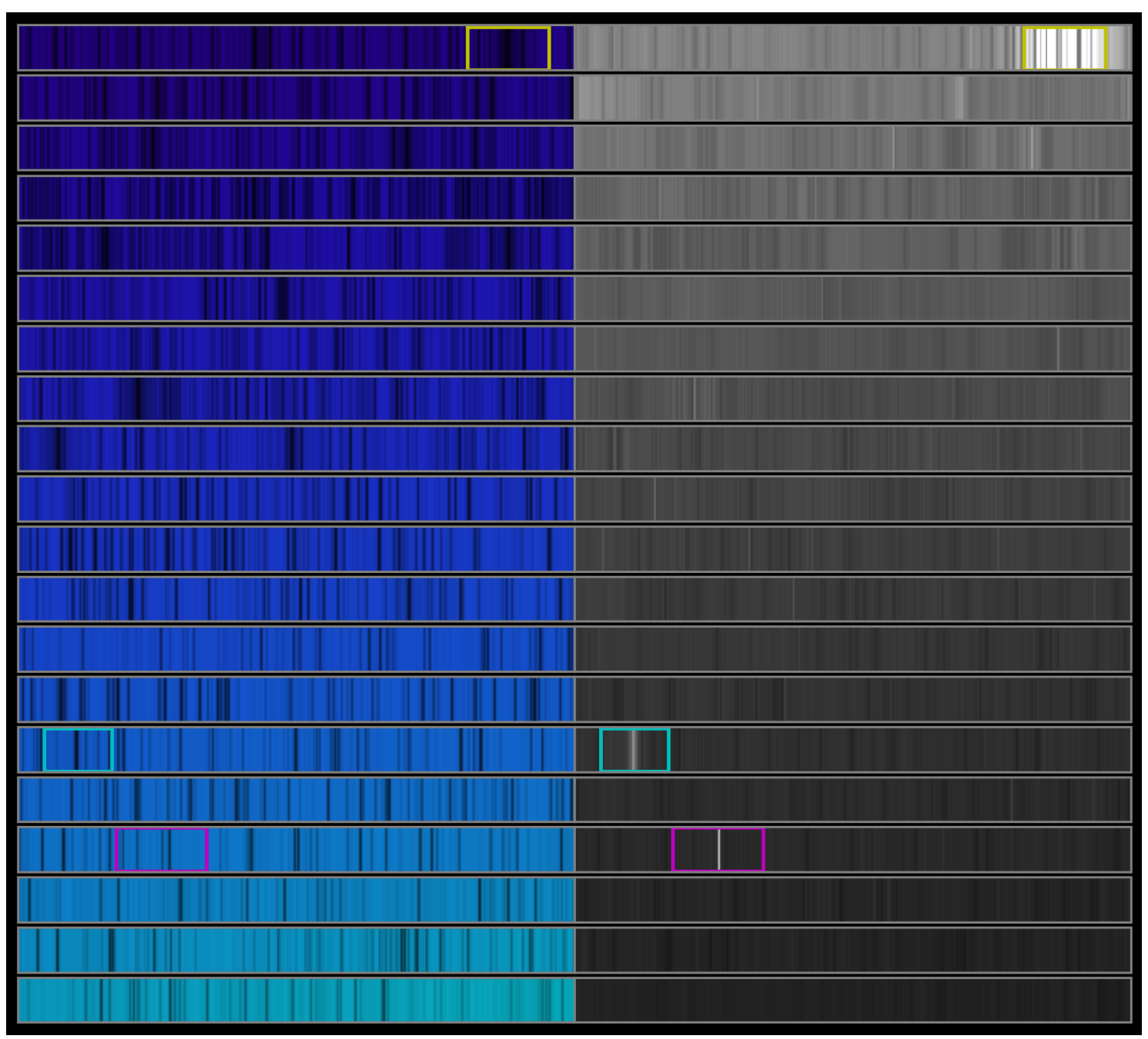

Figure 2.8: Blue part of the second solar spectrum between 4250-4700 $\AA$ taken at $\mu=0.1$, colored to mimic a slit spectrograph observation. ${ }^{18}$ Left: intensity, color brightness normalized to the continuum. Right: linearly polarized spectrum parallel to the solar limb, normalized to the strongest polarization signals of this spectral region. Plot is based on the digital atlas of Gandorfer (2002). With magenta boxes the region between 4603 and $4612 \AA$ is highlighted and a detailed spectrum of this region is plotted in Figure 2.9. The yellow and the cyan box mark the $\mathrm{Ca}$ I line and $\mathrm{Ba} \mathrm{II}_{2} \mathrm{D}_{2}$, respectively. Note that, towards the ultraviolet, also continuum polarization from Rayleigh and Thompson scattering is observable (Fluri \& Stenflo 1999).

\footnotetext{
${ }^{18}$ Inspired by the solar spectrum image of the Kitt Peak atlas shown here: https: //solarsystem.nasa. gov/resources/390/the-solar-spectrum/. A version displaying the complete visible part of the second solar spectrum can be found here: https://commons.wikimedia.org/wiki/File: Second_ Solar_Spectrum. png.
} 
The name tributes the spectral richness of the polarized radiation and its marked difference from the intensity spectrum. Thus, the second solar spectrum is worth exploring just like the intensity solar spectrum (Stenflo \& Keller 1996). An extract of the blue region of the visible second solar spectrum from the survey by Gandorfer (2002) is shown on the right of Figure 2.8.

In Figure 2.8, three lines stand out: $\mathrm{Ca}$ I at $4227 \AA$, Ba II $\mathrm{D}_{2}$ at $4554 \AA$ and $\mathrm{Sr}$ I at $4607 \AA$. The first and the last are transition "twins": both elements belong to the alkaline earths and the lines result from a resonance transition between an upper state level with spectroscopic notation ${ }^{1} P_{0}$ and the ground state ${ }^{1} S_{0}$. This transition has a dipole character and is therefore highly sensitive to scattering, which reflects that Ca I as well as Sr I show the strongest scattering linear polarization signals in the second solar spectrum of the order of $1 \%$ at $\mu=0.1$. However, the formation of the scattering polarization of $\mathrm{Ca}$ I as well as of $\mathrm{Ba}$ II $\mathrm{D}_{2}$ are confined to the chromosphere and thereby unsuitable for photospheric diagnostics.
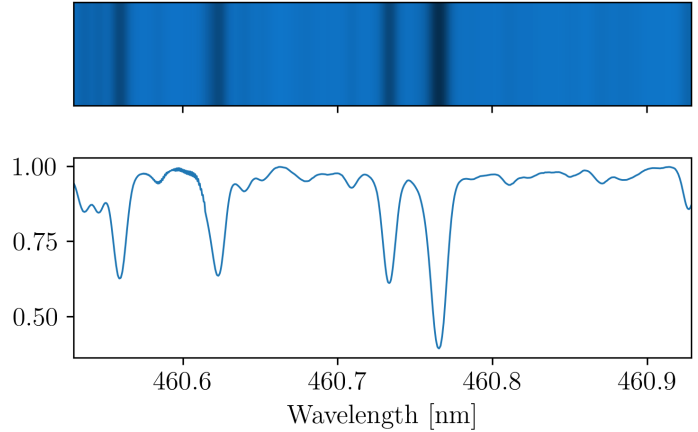
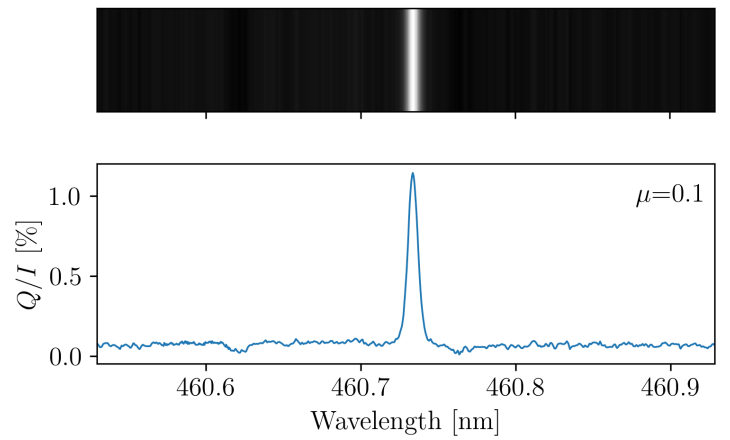

Figure 2.9: The Sr I resonance line within the second solar spectrum. Left: intensity spectrum as shown in the left panel of Figure 2.8. Right: spectrum in the linear polarization parallel to the solar limb as shown in the right panel of Figure 2.8.

The next resonance line which belongs to the same type of transition, but is longer in wavelength and theoretically more suitable for photospheric diagnostics, is $\mathrm{Ba}$ I at $5535 \AA$. The problem with this line is that the scattering polarization amplitude is only about $0.15 \%$. Moreover, Ba I is strongly blended. This means, that several spectral lines are present and overlapping the Ba I line. In complex formation environments, such as the solar atmosphere, this constitutes a challenging modelling problem. In summary: Sr I is at the sweet wavelength spot for formation heights close to the photosphere, has a sufficient scattering amplitude and is one of the simplest lines to model. Figure 2.9 displays the spectrum of Sr I at the limb in more detail, showing a sharp, unblended resonance line with a strong linear polarization signal.

\subsubsection{Scattering polarization at solar disk center}

According to equation (2.23), the linear polarization should be zero when observing the center of the solar disk, i.e. for $\mu=1$. However, the solar photosphere is not homogeneous: spatial inhomgeneities (by radiation, velocity, pressure, etc.) are present due to the 
granulation which breaks the local symmetry and therefore should in principle cause scattering polarization on small spatial scales. The additional presence of magnetic fields and their influence on the Sr I spectral line scattering polarization is discussed in the next section. The rest of this section focus on the radiative inhomogeneities, where we neglect the impact of collisions.

For the disk center case, we chose the quantization axis to be the $z$-axis. For the geometry, see Figure 2.4. According to our chosen setup when deriving the equations for the emission, the observer is located along the $z$-axis, too. In this scenario, $\theta=0$ and $\varphi=0$ apply for the emitted radiation. Further, we define $Q>0$ along the $y$-axis. Consequentially, $Q<0$ along $x$-axis.

In this scenario, there are only six non-zero geometrical components $\mathcal{T}_{q}^{k}(i, \Omega=0)$, see Table A.1 in Appendix A. The emission for each Stokes parameter is therefore:

$$
\begin{aligned}
\epsilon_{I}(\lambda) & =\frac{\eta(\lambda)}{\sqrt{3}}\left(\mathcal{T}_{0}^{0,0}(0,0) J_{0}^{0}+\mathcal{T}_{0}^{2}(0,0) J_{0}^{2}\right)=\frac{\eta(\lambda)}{\sqrt{3}}\left(J_{0}^{0}+\frac{3}{2 \sqrt{2}} J_{0}^{2}\right) \approx \frac{\eta(\lambda)}{\sqrt{3}} J_{0}^{0} \\
\epsilon_{Q}(\lambda) & =\frac{\eta(\lambda)}{\sqrt{3}}\left(\mathcal{T}_{2}^{2}(1,0) J_{-2}^{2}+\mathcal{T}_{-2}^{2}(1,0) J_{2}^{2}\right) \\
& =\frac{\eta(\lambda)}{\sqrt{3}}\left(-\frac{\sqrt{3}}{2}\left(J_{2}^{2}\right)^{*}+\left(-\frac{\sqrt{3}}{2}\right) J_{2}^{2}\right)=-\eta(\lambda) \tilde{J}_{2}^{2} \\
\epsilon_{U}(\lambda) & =\frac{\eta(\lambda)}{\sqrt{3}}\left(\mathcal{T}_{2}^{2}(2,0) J_{-2}^{2}+\mathcal{T}_{-2}^{2}(2,0) J_{2}^{2}\right) \\
& =\frac{\eta(\lambda)}{\sqrt{3}}\left(-\frac{\sqrt{3}}{2} \mathrm{i}\left(J_{2}^{2}\right)^{*}+\left(-\frac{\sqrt{3}}{2}\right)(-\mathrm{i}) J_{2}^{2}\right)=-\eta(\lambda) \hat{J}_{2}^{2} \\
\epsilon_{V}(\lambda) & =0
\end{aligned}
$$

with the notation $J_{ \pm 2}^{2}=\tilde{J}_{ \pm 2}^{2}+\mathrm{i} \hat{J}_{ \pm 2}^{2}$ and assuming the mean intensity is much greater than the anisotropy, i.e. $J_{0}^{0} \gg J_{0}^{\frac{2}{2}}$. According to these equations, the emitted radiation is linearly polarized at disk center if the incident radiation field tensor component $J_{2}^{2}$ is non-zero. Remarkably, the real part of $J_{2}^{2}$ is associated with $Q$ polarization, while the imaginary part of $J_{2}^{2}$ is associated with $U$ polarization (del Pino Alemán et al. 2018).

We write the result for the linear polarization in a more compact way:

$$
\epsilon_{Q}(\lambda)+\mathrm{i} \epsilon_{U}(\lambda)=-\eta(\lambda)\left(\tilde{J}_{2}^{2}+\mathrm{i} \hat{J}_{2}^{2}\right)=-\eta(\lambda) J_{2}^{2} .
$$

It is a reasonable question to ask, what the physical meaning of equation (2.25) is. As the amount of emitted radiation is correlated with $J_{2}^{2}$, a large absolute $J_{2}^{2}$ results in a large amount of polarization. Equation (2.18) provides that $J_{2}^{2}$ depends on the incident radiation field distribution $I^{\text {inc }}\left(\Omega^{\prime}\right)$. Therefore, a specific question is: Which radiation field distribution $I^{\text {inc }}\left(\Omega^{\prime}\right)$ is maximizing $J_{2}^{2}$ and consequentially generates a high degree of negative (i.e., $Q<0$ and $U<0$ ) polarization? ${ }^{19}$ At disk center, $I^{\text {inc }}\left(\Omega^{\prime}\right)$ depends marginally on the polar angle $\theta^{\prime}$. From equation (2.18) we can deduce that an incident radiation field $I^{\text {inc }}\left(\Omega^{\prime}\right)$, which is proportional to the angular dependence $\mathrm{e}^{-2 \mathrm{i} \varphi^{\prime}}$, maximizes $J_{2}^{2}{ }^{20}$ This statement derives from the ortho-normality of the spherical harmonics, i.e.

\footnotetext{
${ }^{19}$ In order to obtain positive polarization, $J_{2}^{2}$ has to be minimized, i.e. largely negative.

${ }^{20}$ For a rigorous mathematical formulation, where the incident radiation is expanded into real-valued spherical harmonics, see Štěpán et al. (2020).
} 
$Y_{2}^{2} \propto \sin ^{2}\left(\theta^{\prime}\right) \mathrm{e}^{2 \mathrm{i} \varphi^{\prime}}$. The azimuthal, or axial, dependence of the incident radiation, which maximizes the absolute of $J_{2}^{2}$, is therefore given by the real and imaginary part of the $Y_{2}^{2}$. We display the real and imaginary part of $Y_{2}^{2}$ in Figure 2.10.

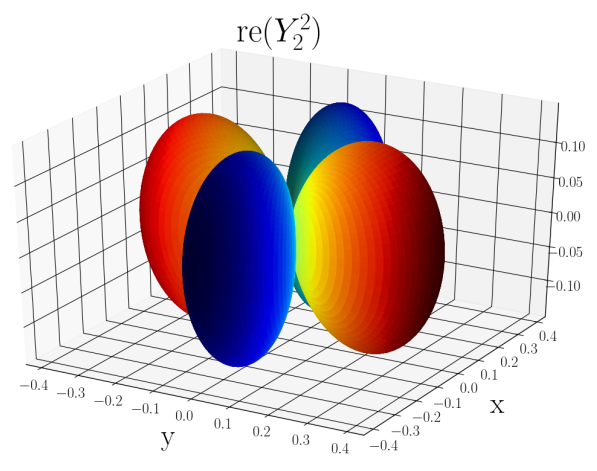

(a) Real part of $Y_{2}^{2}$.

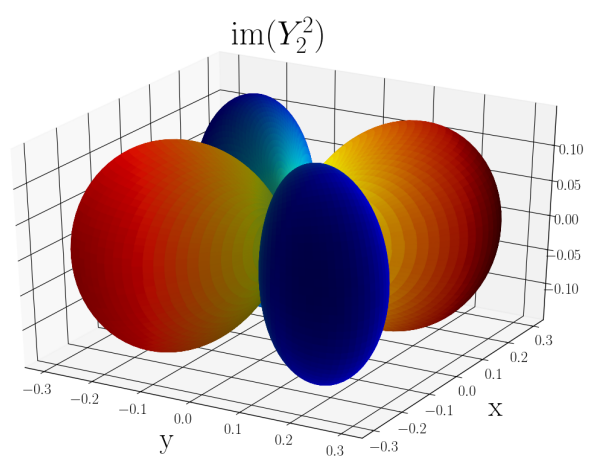

(b) Imaginary part of $Y_{2}^{2}$.

Figure 2.10: Real and imaginary parts of the spherical harmonic component $Y_{2}^{2}$. If the incident radiation field follows the axial distribution the real and imaginary parts, the absolute of the radiation field tensor component $J_{2}^{2}$ is maximized. Therefore, $Q<0$ polarization along the $x$-axis and $U<0$ along the $y=-x$ diagonal is efficiently generated. The blue and red colour represent the region where the $Y_{2}^{2}$ component is negative and positive, respectively.

Figure 2.10 shows that the axial symmetry is broken. Therefore, the $J_{2}^{2}$ is a measure of the axial symmetry break of the incident radiation field. The shape of the distribution is quadrupolar, therefore sometimes $J_{2}^{2}$ is referred to as the quadrupolar component of the radiation field.

In an atmosphere at optical depth unity ${ }^{21}$ of the line center the scattered fractional polarization is then (neglecting collisions, see also equations (9) and (10) in del Pino Alemán et al. 2018)

$$
\frac{Q+\mathrm{i} U}{I}=\frac{\epsilon_{Q}+\mathrm{i} \epsilon_{U}}{\epsilon_{I}}=-\sqrt{3} \frac{J_{2}^{2}}{J_{0}^{0}} .
$$

We graphically show the theoretical relation between the linear polarization as a function of the quadrupolar radiation tensor component $J_{2}^{2}$ in Figure 2.11 .

\footnotetext{
${ }^{21}$ The line optical depth $\tau$ is defined as the integration along the ray path over the absorption coefficient $\eta_{I}$, where the observer is located at the origin at $\tau=0$ (del Toro Iniesta \& Collados 2000). Optical depth unity is where most of the photons originate for a given solar atmosphere.
} 

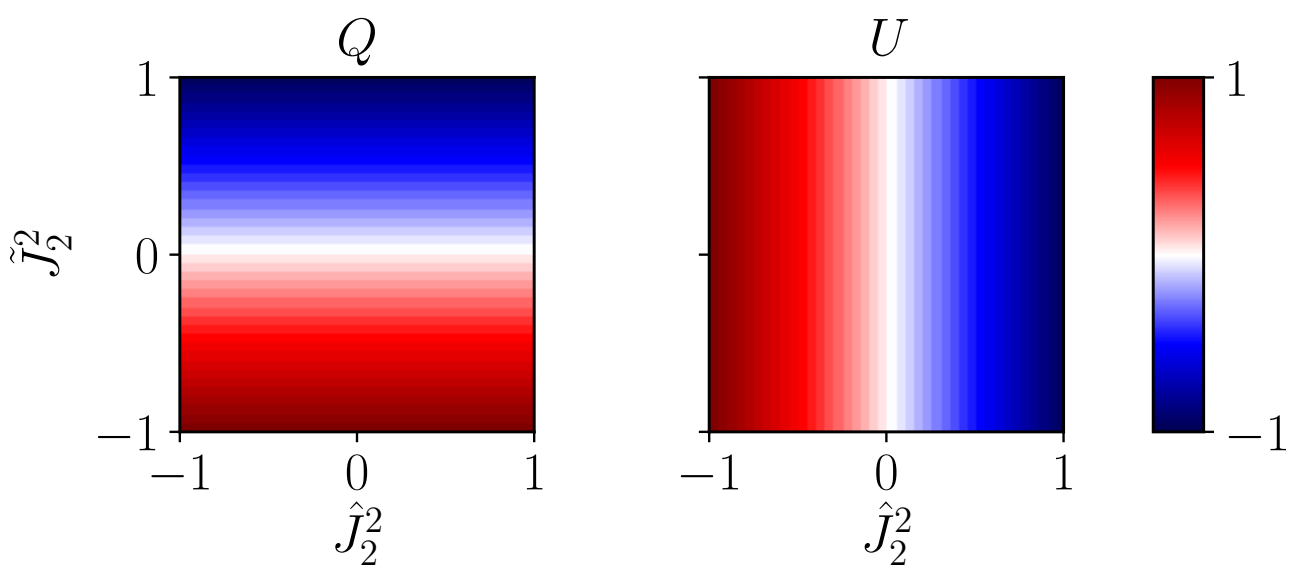

Figure 2.11: Graphical representation of the normalized linear polarization of Sr I at disk center in the absence of a magnetic field, as a function of the (normalized) radiation field tensor component $J_{2}^{2}$. This plot is based on equation (2.26). The amplitude of Stokes $Q$ and $U$ is indicated by the intensity of the colour. The colour indicates the sign.

Trujillo Bueno \& Shchukina (2007) first theoretically proposed that the scattering polarization of $\mathrm{Sr}$ I may be spatially structured with respect to the granulation when observed with high spatial resolution. Later, del Pino Alemán et al. (2018) used a much more detailed 3D magneto-hydrodynamical model of the solar photosphere to solve the RTE in 3D and synthesize the linear polarization signals of Sr I generated by scattering. They found that more polarization is found at the borders of bright, up-flowing granules and dark, down-flowing intergranules, where the horizontal symmetry is broken most strongly by the radiation (and therefore also by macroscopic velocity fields, which induce Doppler shifts).

Until 2018, there was a lack of sufficiently spatially resolved scattering observations in Sr I for $\mu>0.4$. Zeuner et al. (2018) (henceforth, Paper I) showed the first spatially resolved Sr I scattering polarization observation at $\mu=0.6$. These measurements were obtained with a filtergraph. Dhara et al. (2019) obtained measurements with a spectrograph in $\mathrm{Sr}$ I at various limb distances, but even with integration times over $5 \mathrm{~min}$ the scattering amplitude at the disk center was too low to be analyzed. Probably, the signal was further reduced due to the resulting low spatial resolution from the long integration time. At lower limb distances, they found, in contrast to what was found in Paper I and in contrast to what is theoretically expected by del Pino Alemán et al. (2018), stronger polarization amplitudes in granules than intergranules. Zeuner et al. (2020) (from here on Paper II) shows, again using a filtergraph, the first ever evidence for spatially structured scattering polarization in $\mathrm{Sr}$ I at solar disk center. Paper II shows that the scattered radiation is structured according to the quadrupolar component of the radiation field tensor, i.e. $J_{2}^{2}$. Nevertheless, the inconsistency of the results between filtergraph and spectrograph observations still remains.

The quantitative comparison between the scattering polarization amplitude obtained in Paper II and the calculated scattering polarization amplitude given by del Pino Alemán et al. (2018) was conducted by Zeuner et al. 2020b (Paper III, in preparation). If solar evolution is taken into account, the scattering polarization amplitudes from observations and simulations are consistent. 


\subsubsection{The Hanle effect}

In the presence of a magnetic field the scattering polarization is modified - this phenomenon is called the Hanle effect, first theoretically explained by Hanle (1924). Using the oscillator model for atomic electrons, the Hanle effect can be understood classically: like in the case of the Zeeman effect the magnetic field causes a precession of the oscillating electrons around the magnetic field. The trajectory of the electrons produces a rosette pattern, see panel c) in Figure 2.6 (Stenflo 1994; Trujillo Bueno 2001; Landi Degl'Innocenti \& Landolfi 2004). However, the radiative damping given by the lifetime of the upper level, $A_{u \ell}$, competes with the angular frequency of the Larmor precession $\omega_{L}=2 \pi v_{L}=8.7939 \times 10^{6} \mathrm{BG}^{-1} \mathrm{~s}^{-1}$, see equation (2.10). If the former dominates, which means that the Zeeman splitting is negligible compared to the natural line width, the Hanle effect modifies the observed linear polarization Stokes parameters. The observed linear polarization, compared to the magnetic field free case, is a depolarization and a rotation of the polarization plane, see panel b) in Figure 2.6. The critical magnetic field strength $B_{H}$ is defined where the Larmor frequency balances the inverse of the radiative lifetime of the transition. ${ }^{22}$ Thus, for Sr I, the critical magnetic field strength is given by:

$$
B_{H}=\frac{A_{u l}}{8.7939 \cdot 10^{6}} \approx 23 \mathrm{G}
$$

To describe this effect briefly in the quantum mechanical framework, we recall that at disk center the radiation field tensor component $J_{2}^{2}$ induces the component $\rho_{2}^{2}$ of the atomic density matrix and consequentially leads to atomic polarization. According to Table 2.1, $\rho_{2}^{2}$ describes the quantum interference between the $m=1$ and $m=-1$ states. $^{23}$ The coherent superposition of these states generates linear polarization. Applying a magnetic field is like performing a measurement of the atomic state and causes decoherence, i.e. the magnetic field destroys the interference by relaxing the phase relations. The sub-levels are overlapping less with increasing magnetic field strength causing a decrease in emitted linear polarization, see the two right diagrams in Figure 2.3. If the magnetic field is significantly greater than $B_{H}$, which means that the splitting is significantly larger than the natural spectral line width, the two sub-levels add up incoherently and therefore the emitted radiation is unpolarized. This regime is called Hanle saturation. If the splitting of the different components is strong enough to be spectrally resolved, we encounter the Zeeman effect regime.

In the presence of a magnetic field $B$ along the line-of-sight, equation (2.26) is modified by a factor:

$$
\frac{Q+\mathrm{i} U}{I}=-\sqrt{3} \frac{1}{1-2 \mathrm{i} \Gamma} \frac{J_{2}^{2}}{J_{0}^{0}},
$$

with $B / B_{H}=\Gamma$ (Landi Degl'Innocenti \& Landolfi 2004). This equation describes a rotation by an angle $\zeta=\arctan (2 \Gamma)$ in the $\tilde{J}_{2}^{2}-\hat{J}_{2}^{2}$ plane and a depolarization. A graphical representation can be found in Figure 2.12. In Appendix C.1 we demonstrate how to calculate the angle $\zeta$ from observational data. The absence of a rotation angle indicates that any present magnetic field has no preferential orientation in the line-of-sight.

\footnotetext{
${ }^{22} A_{u \ell}=2 \times 10^{8} \mathrm{~s}^{-1}$ for $\mathrm{Sr} \mathrm{I}$.

${ }^{23}$ Note that the states belong to the same atom (Moruzzi \& Strumia 1991).
} 


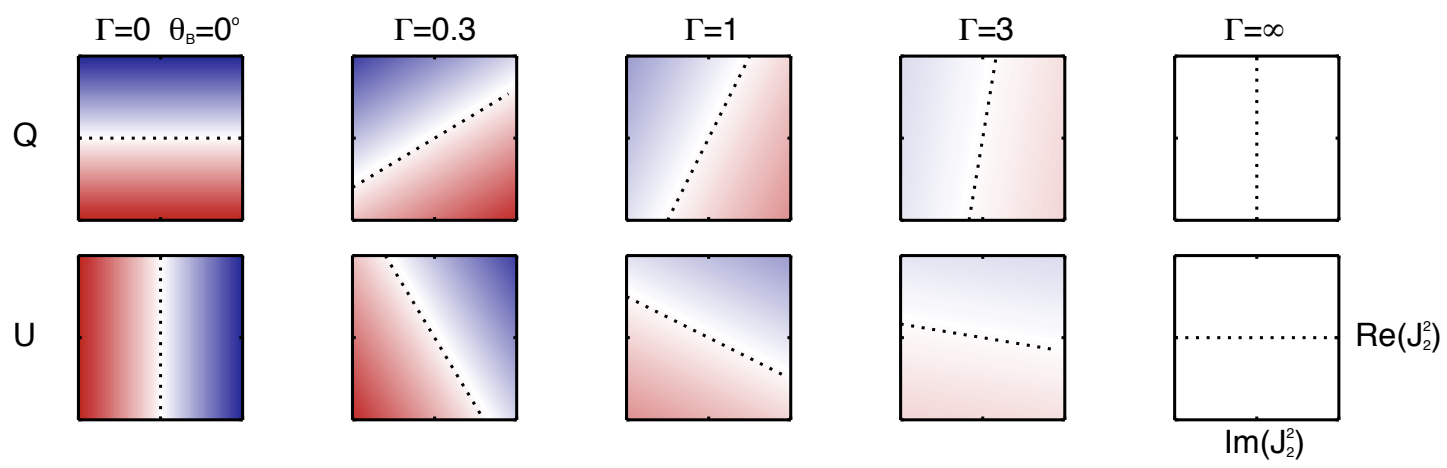

Figure 2.12: Distribution of linear polarization (upper panels: $Q$; lower panels: $U$ ) on the $\tilde{J}_{2}^{2}-\hat{J}_{2}^{2}$ plane, where the colours as in Figure 2.11 indicate the normalized polarization amplitude where the maximum amplitude is reached for $\Gamma=0$. The various panels from left to right display the evolution of this distribution as a function of the normalized magnetic field strength $\Gamma$. In the zero field limit (left-most panels), $Q(U)$ varies linearly with the ordinate (abscissa) as in Figure 2.11. In the presence of an increasing magnetic field along the line-of-sight, there is a rotation of the nodal (dotted) lines and a depolarization according to equation (2.28). Courtesy: Rafael Manso Sainz.

Interestingly, in the case of a small-scale turbulent magnetic field, the amplitude of the scattering polarization is reduced by depolarization, but there is no net rotation of the polarization plane. Assuming an isotropic ${ }^{24}$ micro-turbulent magnetic field, Trujillo Bueno \& Manso Sainz (1999) deduced the dimensionless depolarization factor $\mathcal{H}^{(2)}$. A micro-turbulent magnetic field implies that the magnetic field changes its polarity several times within the photon mean free path. In this case, equation (2.28) changes to

$$
\frac{Q+\mathrm{i} U}{I}=-\sqrt{3} \mathcal{H}^{(2)} \frac{J_{2}^{2}}{J_{0}^{0}},
$$

with $\mathcal{H}^{(2)}=\frac{1}{5}\left(1+2 /\left(1+\Gamma^{2}\right)+2 /\left(1+4 \Gamma^{2}\right)\right)$ and assuming the collisionless limit. There are a few consequences caused by replacing $\frac{1}{1-2 \mathrm{i} \Gamma}$ with $\mathcal{H}^{(2)}$. First, $\mathcal{H}^{(2)}$ is a real number and thereby does not rotate the linear polarized scattered radiation in the $\tilde{J}_{2}^{2}-\hat{J}_{2}^{2}$ plane. Further, in the zero-field limit, $\mathcal{H}^{(2)}=1$, and equation (2.26) is recovered. In the saturation regime, i.e. $\Gamma \rightarrow \infty, \mathcal{H}^{(2)}=1 / 5$. In contrast to the line-of-sight oriented magnetic field case described by equation (2.28), equation (2.29) implies that the scattering polarization amplitude is greater than zero even in the case of large magnetic fields. However, the sensitivity of the Hanle effect to a micro-turbulent magnetic field is limited to cases where $1>\mathcal{H}^{(2)}>1 / 5$. In order to determine $\mathcal{H}^{(2)}$ (and thus $|\Gamma|$ ) the zero-field scattering polarization amplitude has to be known. ${ }^{25}$ This requires a precise modeling of the scattering polarization amplitude, which dependents on the conditions of the solar atmosphere. Zero-field and observed scattering polarization amplitude have to be known to an accuracy where it is possible to estimate $\mathcal{H}^{(2)}$ within the limited range of 0.2 and 1 . Further observations and theoretical modeling of scattering polarization in spectral lines at the center of the solar disk has to be done to achieve this goal in the future.

\footnotetext{
${ }^{24}$ Isotropic in the sense that the distribution of magnetic field orientations is isotropic.

${ }^{25}$ Note that the polarity of $B$ is not uniquely determined by $\Gamma^{2}$.
} 
Moreover, the Hanle effect is sensitive to weaker magnetic fields than the Zeeman effect. Therefore, the Hanle effect is a complementary tool to the Zeeman effect to diagnose astrophysical magnetic fields (see overviews by, e.g. Trujillo Bueno et al. 2005; Stenflo 2013). A disadvantage is that the Hanle effect is not sensitive to horizontal magnetic fields, i.e. in case the magnetic field vector lies in the plane perpendicular to the scattering direction. It was also found by Faurobert-Scholl et al. (1995) that the depth dependence of the turbulent magnetic field is poorly constrained.

In the real Sun, the situation is much more complicated, as the symmetry is not only broken by the radiation field, but also by velocity fields (del Pino Alemán et al. 2018). Further, not only the magnetic field is depolarizing, but also collisions (Landi Degl'Innocenti \& Landolfi 2004; del Pino Alemán et al. 2018). Collisions, which are usually isotropic, ${ }^{26}$ thermalize the atoms, i.e. they populate all Zeeman sub-levels equally and destroy atomic polarization. Another effect of collisions is that the critical magnetic field for the onset of the Hanle effect is modified. A collisionrate $\delta$ increases the critical magnetic field strength $\left(B_{H}\right.$ in the collisionless regime), now denoted by $B_{H}^{\prime}$ (del Pino Alemán et al. 2018):

$$
B_{H}^{\prime} \approx\left(1+\delta / A_{u \ell}\right) B_{H} .
$$

With increasing depth, the collisional rates also increase. For $\mathrm{Sr}$ I at disk center, where deeper layers than at the limb are observed, $B_{H}^{\prime} \approx 100 \mathrm{G}$. This implies that scattering polarization is still observable even if the photospheric turbulent magnetic fields are of the order of $100 \mathrm{G}$.

Interpreting scattering polarization in terms of the Hanle effect is a complex task which usually relies on modeling, where the zero-field scattering polarization is calculated and compared to the observed linear polarization. Of course, this task is even more challenging in the case of spatially resolved scattering measurements, as it would require a detailed determination of the atmosphere spatially by, for example, other spectral lines and the knowledge of the collision rates for Sr I, or any other scattering sensitive spectral line.

Another approach is to use the differential Hanle technique (Stenflo et al. 1998), which is based on comparing spectral lines with different sensitivities to the Hanle effect. However, it is a prerequisite that the spectral lines are formed in the same atmospheric conditions. As pointed out in Sect. 2.3.2.1, the Sr I line is formed in the photosphere, without similar lines sensitive to scattering in direct spectral vicinity. Therefore, the differential Hanle technique is unsuitable for SrI.

${ }^{26}$ Recently, Qutub et al. (2020) showed that anisotropic collisions can create circular scattering polarization. 


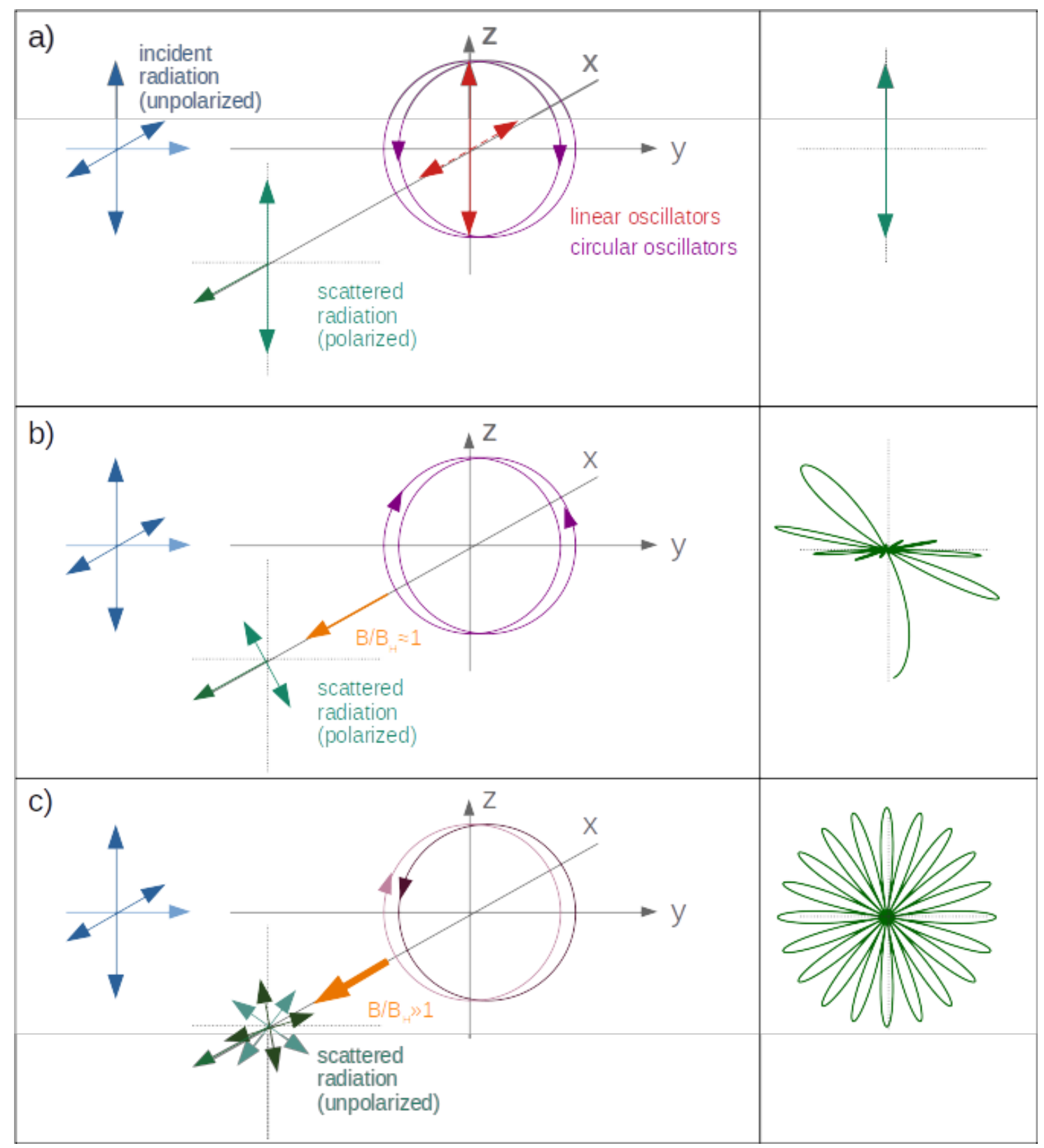

Figure 2.6: Classical description for scattering light at a classical oscillator with and without considering a magnetic field (left). The oscillator trajectories are shown on the right (e.g., Jackson 1998; Mitchell \& Zemansky 2009). Consider the atom as a dipole oscillator under $90^{\circ}$ unpolarized illumination, as in panel a). The two uncorrelated perpendicular components of the incident radiation excite two uncorrelated oscillations of the oscillator, where one of them is observed pole-on. The pole-on linear oscillator radiates zero power towards the observing direction, therefore the observed scattered radiation is completely linearly polarized. b) Modified scattering polarization in the presence of a weak magnetic field (Hanle effect), where the linear oscillator is decomposed into two circular oscillators, like in Figure 2.4. The coherence of the circular oscillators is disturbed and the resulting scattering polarization is rotated. c) A strong magnetic field destroys the coherence between the two circular oscillators and the scattered light is completely depolarized (saturated Hanle regime). See Sect. 2.3.2.3 for the quantum mechanical description. 


\subsection{Ground-based fast solar spectropolarimetry}

Measuring the polarization state of light as a function of wavelength is referred to as spectropolarimetry. Polarized light cannot be measured directly since the light recording detectors (cameras) are only sensitive to intensities. Therefore, polarization has to be converted into intensity. This conversion is called modulation and is performed by a modulator based on retarders and a polarization analyzer. Consequentially, the three basic components of a spectropolarimeter are the wavelength discriminator, the modulator, and the camera. The polarimeters used for the spectropolarimetric data acquisitions in this thesis are the Fast Solar Polarimeter (FSP, Iglesias et al. 2016), and a modification of FSP, called FSP 2. FSP is based on fast temporal modulation and a synchronized fast scientific CCD camera, while FSP 2 is based on spatio-temporal modulation synchronized with a fast CMOS camera.

To study polarization signals on small spatial scales, a good spatial resolution is required. Therefore, we used FSP and FSP 2 in filtergraph mode, although this leads to compromises in the spectral resolution. More particularly, Fabry-Pérot etalons discriminate the wavelengths for the data used in this thesis. Unlike for Zeeman signals, where it is essential to capture the spectral shape (see Sect. 2.3.1), scattering polarization is to first order proportional to the line shape itself (see Sect. 2.3.2), and lower spectral resolution may result in higher signal-to-noise ratios. This in turn helps detecting faint scattering polarization signals.

However, most spectropolarimetric observations are photon starved, and to reach high sensitivity in polarimetric measurements one has to additionally average temporally and/or spatially. On the other hand, the quiet solar photosphere evolves in a matter of minutes, thus temporal averages lead to lower spatial resolution. Hence, there is a constant trade-off between spatio-temporal resolution and polarimetric sensitivity, which can be optimized by collecting more photons by large aperture solar telescopes (see for example review by Iglesias \& Feller 2019).

FSP and FSP 2 are polarimeters designed to be attached to ground-based solar telescopes, which usually provide the largest available apertures. They provide high-sensitivity $(<0.1 \%)$ and high-resolution $(<1$ arcsec $)$ spectropolarimetric data by minimizing polarimetric errors and image degradations introduced by (Earth's) atmospheric seeing.

In this chapter, we briefly review the particularities of the FSP measurement principle. We describe the improvements achieved with FSP 2 and the main post-processing steps which reduce noise in the FSP 2 polarimetric data. We also briefly discuss the working principle of Fabry-Pérot etalons.

\subsubsection{Stokes polarimetry by temporal modulation}

To measure all four Stokes parameters of light a modulator changes the polarization state of the incoming light in a predetermined way (see for example Stenflo 1994; del Toro Iniesta \& Collados 2000). The modulated polarization state is analyzed and the intensity is recorded with a camera. The weighted combination of the measured intensities returns the polarization state of the incoming light.

The modulator of FSP is based on two ferro-electric liquid crystals (FLCs) combined with two static retarders, followed by a polarizing beam splitter cube as an analyzer. This 
configuration is called single-beam polarimetry. ${ }^{27}$ The modulation state is changed by switching the optical axis orientation of the FLCs between two discrete angles, i.e. two different states per FLC (see next section for more details, and for a full characterization of the modulator, see Iglesias 2016). The minimum number of modulation states for a full characterization of the Stokes vector is four. Four intensity measurements $\hat{I}$ resulting from four modulation states encoded in the $4 \times 4$ modulation matrix $\boldsymbol{O}$ are connected to an observed Stokes vector $\boldsymbol{I}$ via

$$
\hat{\boldsymbol{I}}=\boldsymbol{O I} \text {. }
$$

Each row of $\boldsymbol{O}$ represents one state of the modulator given by the FLC angles. As the angles are changed consecutively with time and consequentially each of the four intensity measurements are separated in time, the FSP modulation is a temporal modulation, see next section for more details.

Ideally, the input Stokes vector $\boldsymbol{I}$ is recovered by the demodulation matrix $\boldsymbol{D}=\boldsymbol{O}^{-1}$. However, the elements of the modulation matrix are usually the result of a calibration procedure, where known input states are fed into the polarimeter. The input states are usually generated with a combination of achromatic quarter wave retarders and a polarizer. By minimizing the difference between the input states and the response of the polarimeter to them, the elements of the matrix $\hat{\boldsymbol{O}}$ are calculated. However, differences in the modulation matrix $\hat{\boldsymbol{O}}$ from the true matrix $\boldsymbol{O}$ decreases the polarimetric accuracy. The calibration procedure also leaves residual uncertainties in the modulation matrix, mainly due to measurement noise or instabilities, i.e. small changes in the polarimeter response between calibration and science data recording. Therefore, the calibration is usually imperfect and the input Stokes vector may only be approximated with

$$
I \approx I^{\prime}=\hat{D} \hat{I} .
$$

Ideally, the calibration optics are placed as early in the optical train of the solar telescope as possible, as every optical element after the calibration optics counts as a part of the polarimeter and is therefore calibrated (e.g., del Toro Iniesta \& Collados 2000). For practical reasons, this means that the calibration optics should be placed in front of the solar telescope, which is usually not possible. Therefore, solar polarimetric data may suffer from telescope polarization and telescope induced cross-talk between the Stokes parameters.

The latter may be corrected by applying a known matrix of the telescope (the telescope model) to the observed Stokes parameters. Another option, i.e if a telescope model is unavailable, is to apply a heuristic cross-talk correction technique. For this, it is assumed that the strongest polarization signatures in the linear polarization signals belong to Stokes $V$. But, correcting any cross-talk between $Q$ and $U$ is very challenging with this technique. A heuristic cross-talk correction technique is used for the polarimetric correction in this thesis (see Sects. 3.3.1.2, 3.3.2.2 and 4.7.2).

In the most benign type of telescope polarization, just an offset is added to the polarimetric signals, which can be removed by assuming that the mean polarization at solar disk center is zero and therefore the mean polarization present in disk center data for each Stokes parameter may be subtracted. However, in combination with a non-linear response

${ }^{27}$ Note that this results in the loss of about half the available photons. 
of the camera, telescope polarization induces spurious signals proportional to Stokes $I$. A possible correction method of this error is explained in Sect. 2.4.3.2.

\subsubsection{The Fast Solar Polarimeter}

Ground-based solar spectropolarimetry is affected from seeing induced by the Earth's atmosphere. Seeing is produced by changes of the refractive index of the atmosphere, which causes wavefront aberrations (deformations). In solar observations, the change of the refractive index is driven by temperature fluctuations. The temporal average of randomly distorted wavefronts reduces the image quality, the RMS contrast for example. Moreover, polarimetry based on temporal modulation depends on a temporally stable input Stokes vector. If this assumption is violated, spurious signals in the demodulated Stokes vector image are present in the form of seeing induced cross-talk (SIC). Lites (1987) found that SIC increases with the RMS of the image motion, while it decreases with increasing modulation frequency, until it becomes negligible for modulation frequencies above $400 \mathrm{~Hz}$. The prominent high-sensitivity ground-based polarimeter ZIMPOL (Povel et al. 1990; Keller et al. 1994b; Ramelli et al. 2010) is based on modulation frequencies in the range of 1-10 kHz. However, the camera read out is slower by orders of magnitude. Krishnappa \& Feller (2012) showed that a modulation frequency of about $100 \mathrm{~Hz}$ is sufficient to keep the SIC level below $10^{-4} \cdot I$.

Fast cameras, such as the Peltier-cooled high-frame rate pnCCD (charge-coupled device) camera designed for FSP (Iglesias et al. 2016), can be read out at this frequency. At the same time the noise and in particular the read out noise must be kept as low as possible to enable high-sensitivity polarimetric measurements. Moreover, the exposure time of one image frame should be extended as long as possible to harvest as many photons as possible. The product of frame rate and exposure time is called duty cycle, and the optimum is $100 \%$. The FSP camera was designed to have a duty cycle of more than $98 \%$. Note that with a modulation frequency of $100 \mathrm{~Hz}$ the frame rate has to be $400 \mathrm{fps}$ to measure four polarization states within one modulation cycle. The advantages of a fast camera read out with high duty cycles are the possibility to decide on the cadence of the observation based on the observationally present dynamics and required signal-to-noise ratio after the observation has been carried out and to perform post-processing image restoration to increase the image quality (for a review, see Löfdahl 2002). The potential of image restoration typically relies on spectropolarimetric imaging with higher signal-to-noise ratios (Iglesias et al. 2016). Because of a too low signal-to-noise ratio, image restoration was not applied to most of the data in this thesis.

However, the main idea of FSP is to combine fast modulation with fast, high duty cycle and low-noise cameras to suppress SIC and provide high-sensitivity polarimetric data which at the same time is recorded at high-cadence. FSP has been designed to respond achromatically (work efficiently in a broad range of wavelengths) from 400-800 nm and has been fully characterized in Iglesias (2016).

Paper I is based on FSP data, and the observation specifications as well as the data reduction steps can be found there and therefore will not be repeated here. 


\subsubsection{The Fast Solar Polarimeter 2}

For FSP 2, the FSP concept and modulator were adopted but two components of the instrument were replaced. By replacing the $264 \times 264$ pixel pnCCD camera by a commercially available, but customized (temperature controlled) $4 \mathrm{k} \times 3 \mathrm{k}$ pixel CMOS (complementary metal-oxide-semiconductor) sensor based camera, the field-of-view is significantly extended. Section 4.7.2 includes a short summary of the camera characteristics. A detailed CMOS camera characterization is part of Kamal Sant's thesis at the Max Planck Institute for Solar System Research, Göttingen, currently in progress.

Additionally, the modulator is combined with a novel polarizing beam splitter (PBS) which transfers two orthogonally polarized images of the identical solar scene onto the same sensor. The strictly simultaneous measurement of both orthogonal polarization states separated in space (using either two detectors or two parts of the same detector) is called dual-beam polarimetry (Lites 1987). This spatial modulation is added to the temporal modulation by the modulator, therefore FSP 2 operates with a spatio-temporal modulation.

As a part of this thesis the PBS is characterized and the potential of the dual-beam configuration is investigated. In the next two sections we will give an overview of the PBS characterization and how FSP 2 data benefit from the dual-beam configuration.

Paper II makes use of FSP 2 data, and the details of the observation and the data reduction steps ${ }^{28}$ can be found in Sect. 4.7.1 and 4.7.2. However, one of the data reduction steps involved the correction of cross-talk between the Stokes parameters. We describe the details of the correction in Sect. 2.4.3.2.

Some results of this chapter have been presented in the Deliverable 60.6 report for the SOLARNET project.

\subsubsection{Dual-beam polarimetry}

This section describes the polarizing beam splitter (PBS) for the dual-beam configuration, and the test results demonstrating its performance. In contrast to the single-beam configuration of FSP, the dual-beam configuration increases the polarimetric efficiency by about a factor of $\sqrt{2}$, as all the available light is used for the polarization analysis. In addition the strictly simultaneous recording of the two beams further suppresses any residual cross-talk between intensity and linear or circular polarization.

In the following, we describe the design and show the test results of the functionality of the PBS. Figure 2.13 shows the schematic layout of the PBS. This high-precision optical device has been designed by the Max Planck Institute for Solar System Research, Göttingen, in collaboration with the Canadian optics manufacturer LightMachinery Inc. The ray bundle shown in red enters the fused silica prisms of the PBS at normal incidence, but at an angle of $32^{\circ}$ with respect to the sensor plane normal. After splitting at the second face, the orthogonally polarized $\mathrm{s}$ and $\mathrm{p}$ beams ${ }^{29}$ undergo two total internal reflections before exiting the PBS normal to the exit face as two parallel beams covering the entire sensor area of about $22.5 \times 16.9 \mathrm{~mm}^{2}$. The paths of both the $\mathrm{s}$ and $\mathrm{p}$ beams in the PBS are symmetric and the beams travel exactly the same optical distance. This ensures that no differential optical effects are introduced between the beams, and that both beams are

\footnotetext{
${ }^{28}$ The FSP 2 data reduction pipeline was developed as part of this thesis.

${ }^{29}$ For orthogonally and parallel polarized beams with respect to the plane of incidence.
} 

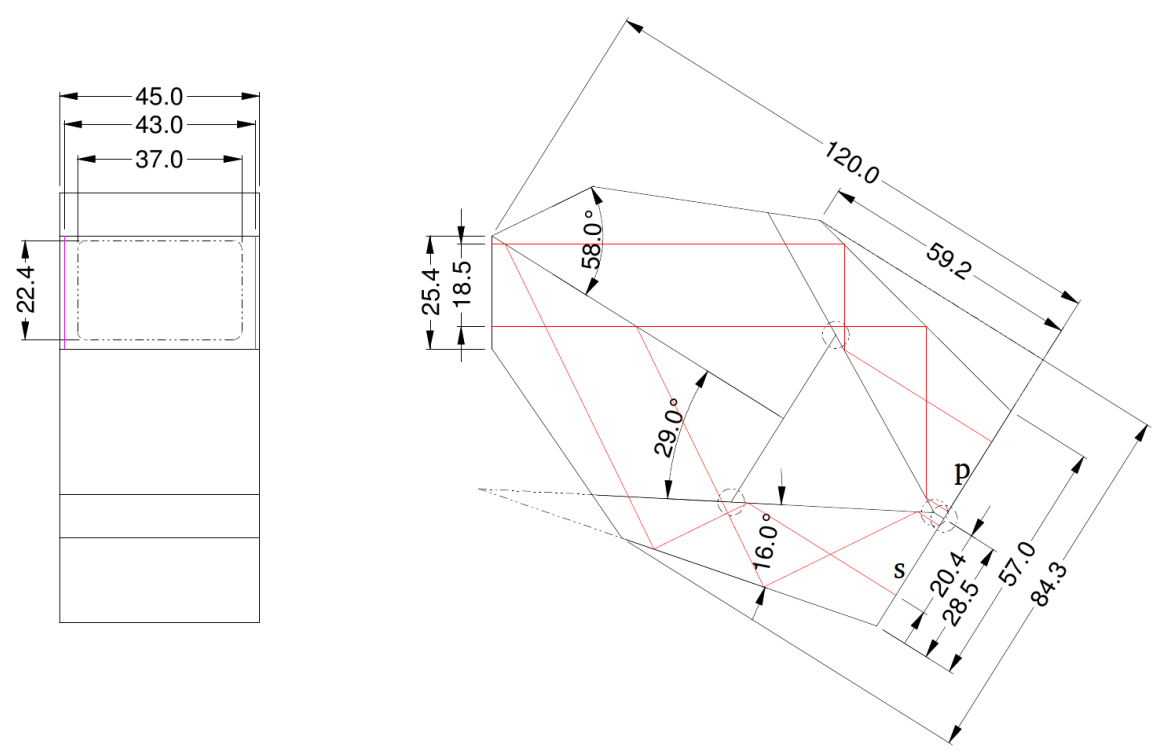

Figure 2.13: Manufacturing drawing of the polarizing beam splitter (PBS) for the dualbeam configuration of the FSP modulator; top view onto the beam splitting plane (right) and side view onto the PBS entrance aperture (left). The red lines indicate the light path. Image courtesy: LightMachinery Inc.

exactly in focus on the sensor plane. The normal beam incidence at the entrance and exit faces avoids any wavelength dependent refraction effects. Further, the PBS significantly extends the spectral working range of FSP. Table 2.2 compiles the main requirements of the PBS.

Table 2.2: Main requirements of the PBS for the FSP dual-beam configuration.

\begin{tabular}{ll}
\hline \hline Specification & Value \\
\hline Spectral working range & $390-860 \mathrm{~nm}$ \\
s and p beam intensity difference for unpolarized light & $<5 \%$ \\
Extinction ratio & $1: 20$ (req.), $1: 100$ (goal) \\
Absolute wavefront error & $<\lambda / 4 \mathrm{P}-\mathrm{V}^{30}$ at $630 \mathrm{~nm}$ \\
Differential wavefront error between s and p beams & $<\lambda / 10 \mathrm{RMS}$ at $630 \mathrm{~nm}$ \\
Glass material & Fused silica \\
AR coatings for entrance and exit faces & $<1 \%$ reflectivity \\
\hline
\end{tabular}

The PBS is housed in an anodized aluminium enclosure to avoid stray light outside of the beam path, see Figure 2.14.

First, we tested the extinction ratio in the required wavelength range between $400 \mathrm{~nm}$ and $850 \mathrm{~nm}$. For the setup, we used a halogen lamp whose light is diffused, collimated, filtered with narrow bandpass interference filters $(\sim 2 \mathrm{~nm}$ bandwidth) and polarized by a Glan-Thompson (GT) polarizer (extinction ratio $\sim 10^{4}$ ). As a target, a square aperture (with a length of $6 \mathrm{~mm}$ ) is used and imaged with two plane-convex lenses in a telecentric setup,

${ }^{30}$ Peak-to-valley. 


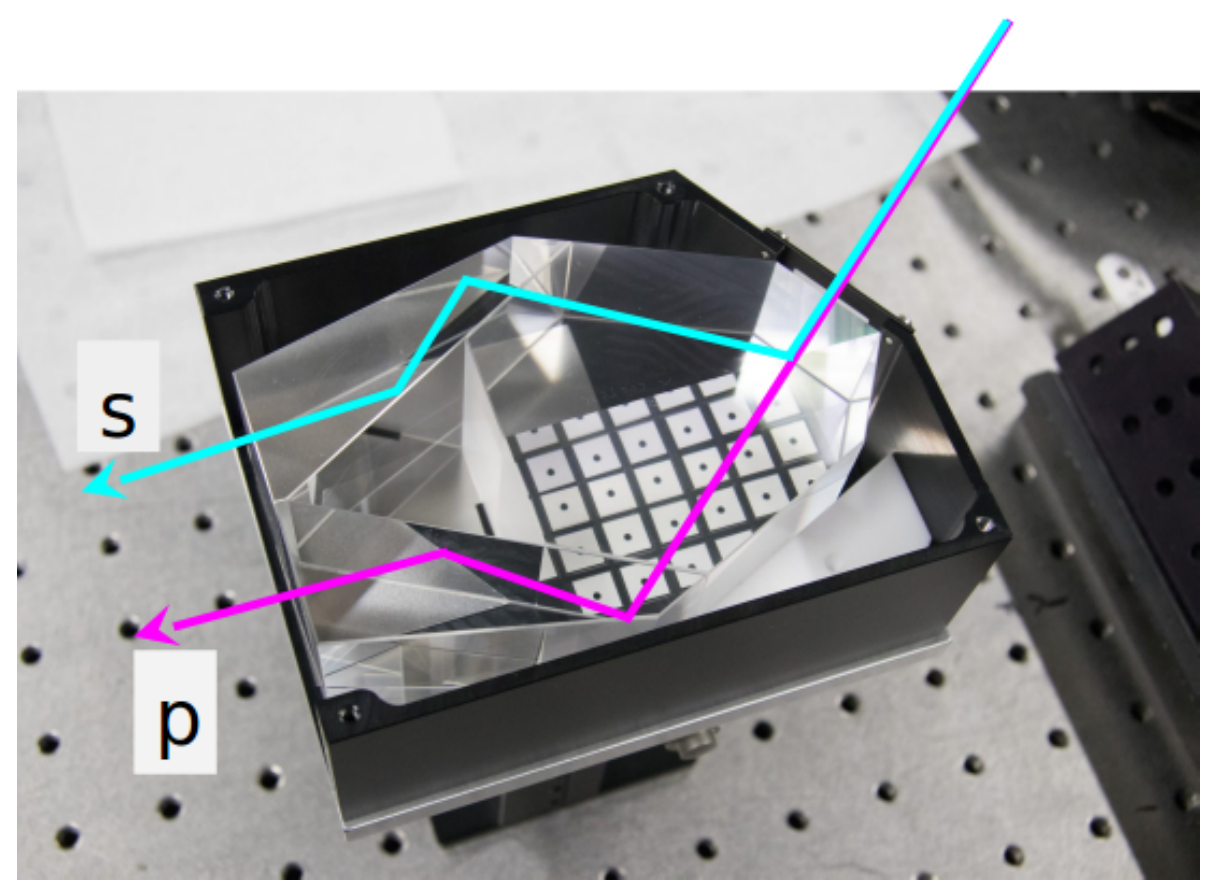

Figure 2.14: Photograph of the PBS mounted in an anodized aluminum housing. To prevent the PBS from changing position relative to the detector, it is glued after careful alignment to the housing.

having an aperture stop in between to reduce aberrations. The PBS is placed after the polarizer and the FSP pnCCD is used as a detector very close to the output of the PBS to avoid stray light. Both images, i.e. p-polarized and s-polarized, are fully visible on the pnCCD sensor. Saturation of the chip is avoided by using a maximum light level of about half of the dynamic range of the pnCCD. See Figure 2.15 for the measurement setup.

The measurement was carried out as follows: For each of the four filters within the required working range, we recorded one dark image for $25 \mathrm{~s}$ by blocking the incoming light at the aperture stop. Thereby, we ensure to remove residual light present in the laboratory as well as the dark current of the pnCCD if subtracted from the measurement image. Following this, we change the polarizer angle $\alpha$ with steps of $\Delta \alpha=10^{\circ}$ and recorded with an integration time of $25 \mathrm{~s}$ the intensity, having in total 20 measurement points.

The recorded images are then corrected for frame-transfer smearing (Iglesias et al. 2015), common mode and dark image. All frames for each wavelength and polarizer position are averaged, resulting in a maximum SNR of $\sim 350$ per pixel. Within each of the images an area of $100 \times 50$ pixel $^{2}$ for each output channel is averaged. To obtain the extinction ratio, we divide the maximum intensity by the minimum intensity of each channel.

An example of the measured intensities $I_{\alpha}$ in both (p-and s-polarized) channels for $400 \mathrm{~nm}$ is given in Figure 2.16. The extinction ratio $R^{2}$ is obtained by calculating $R_{s, p}^{2}=\frac{\max \left(I_{s, p}(\alpha)\right)}{\min \left(I_{s, p}(\alpha)\right)}$ for each channel. The lower limit of the calculated extinction ratios $R_{\min }^{2}=\min \left(R_{s, p}^{2}\right)$ for each wavelength position are given in Table 2.3 below. We found that the minimum extinction ratio of 1:49 meets the specifications very well.

The estimation of the imaging quality is done by obtaining flatfield corrected images 


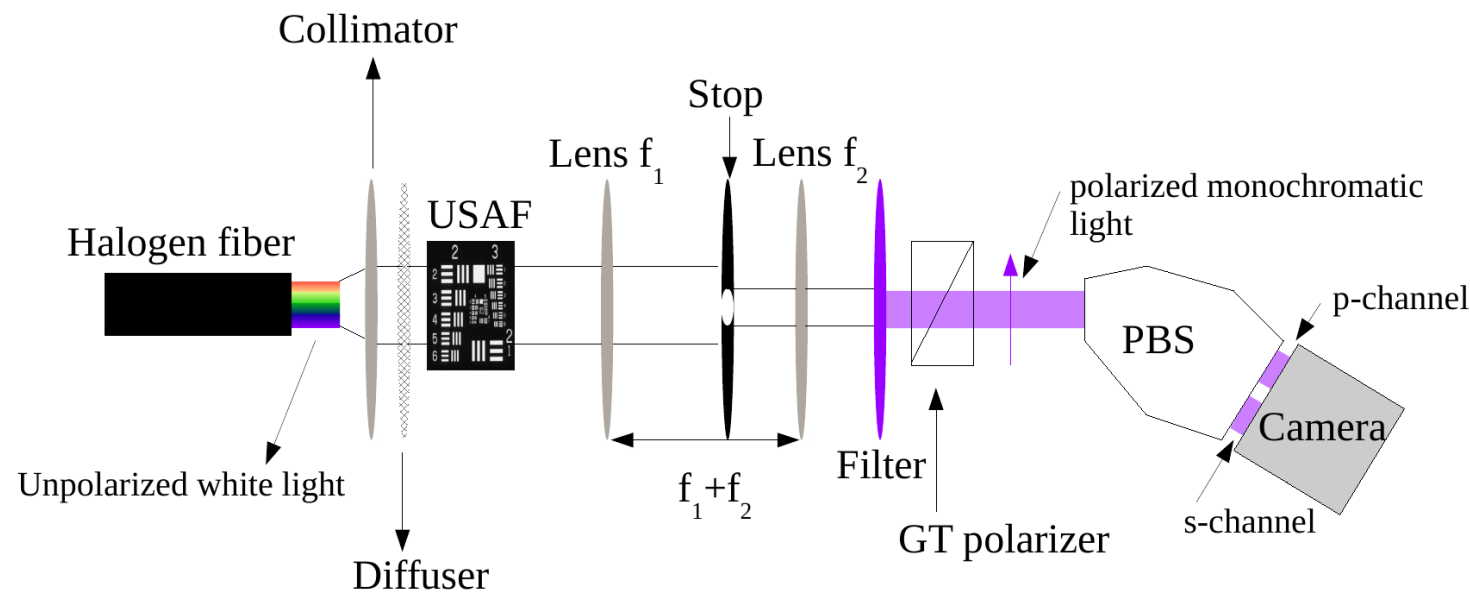

Figure 2.15: Schematic of the measurement setup for the polarimetric characterization of the PBS. This setup allows us to study the polarimetric performance of the PBS with unpolarized and polarized light avoiding as much stray light as possible. As a detector the FSP pnCCD camera is used. For the measurements of the extinction ratio, the USAF target is removed. For the telecentric imaging two plano-convex lenses with $f_{1}=600 \mathrm{~mm}$ and $f_{2}=300 \mathrm{~mm}$ are used.

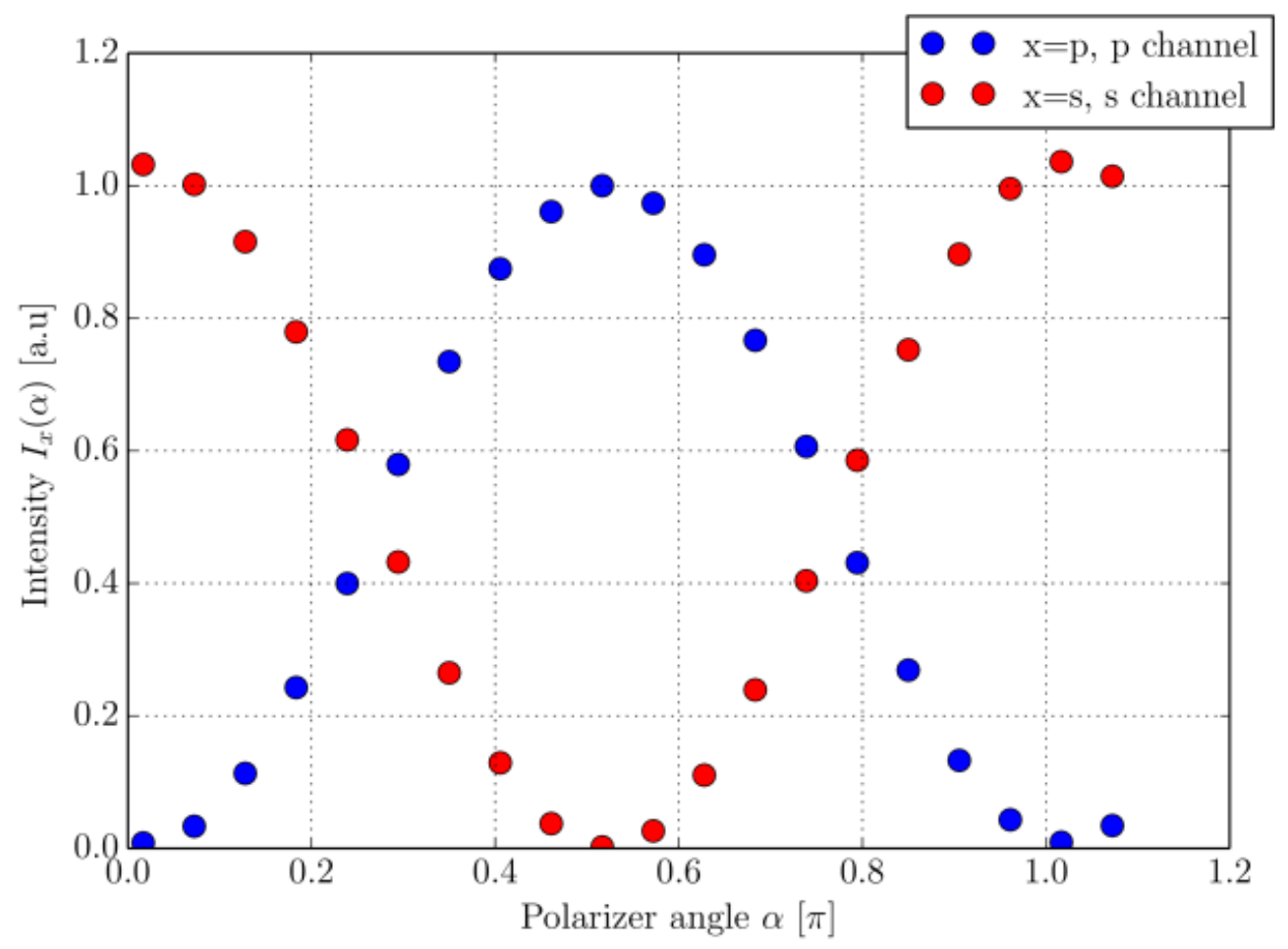

Figure 2.16: Normalized measured intensities $I_{\alpha}$ for the p-polarized and s-polarized output channel of the PBS with respect to the polarizer angle $\alpha$ at a wavelength of $400 \mathrm{~nm}$. Both are normalized to the maximum intensity of the p-channel. 
Table 2.3: Obtained extinction ratios with values not less than 1:49 are found within the required wavelength range. These results meet the specifications, see Table 2.2.

\begin{tabular}{lcccc}
\hline \hline Wavelength [nm] & 400 & 517 & 601 & 850 \\
\hline Extinction ratio $R_{\min }^{2}$ & $1: 111$ & $1: 71$ & $1: 344$ & $1: 49$
\end{tabular}
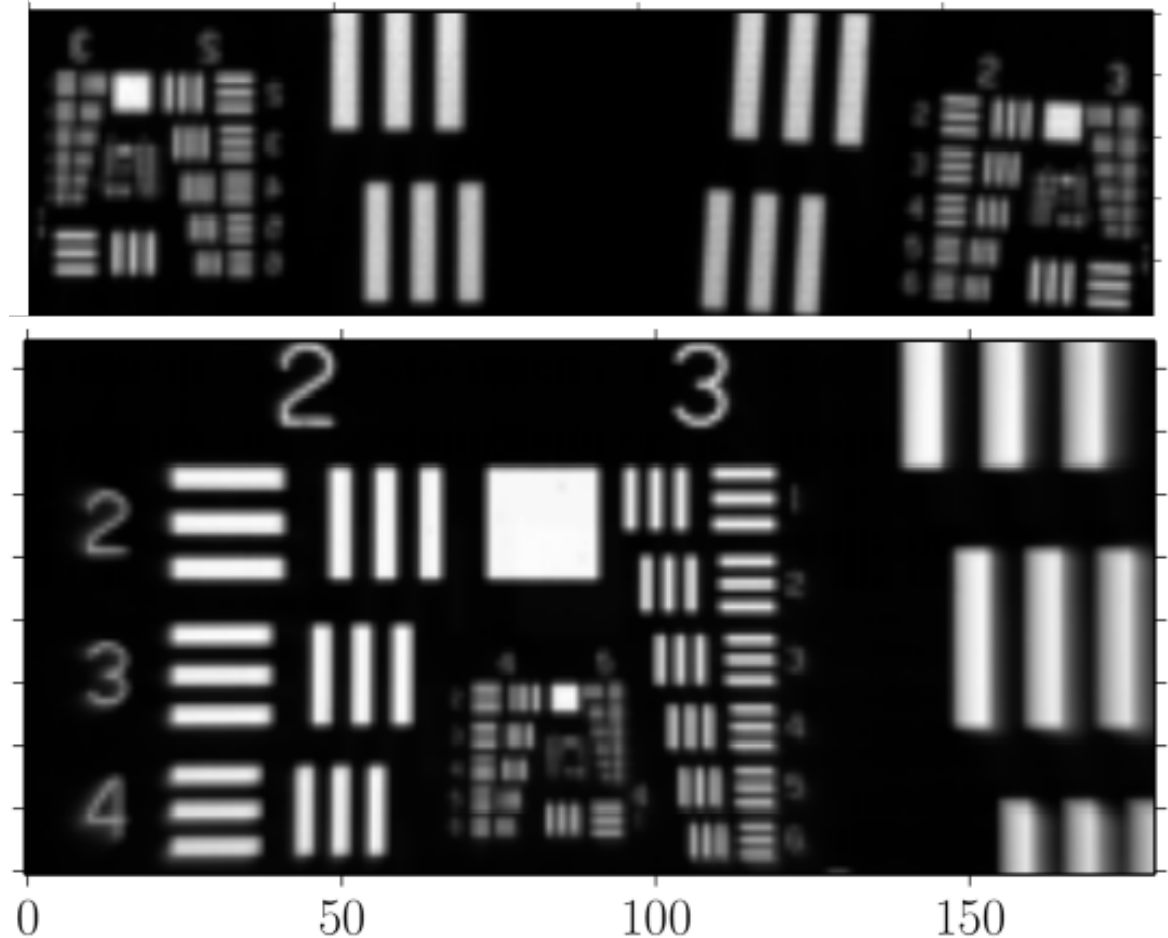

Figure 2.17: Flatfield corrected images of the focused USAF target. Top panel: image with PBS. Groups 2 and 3 (numbers on top) are displayed, last resolved element in the s- and p-polarized sub-images is group 2 element 3 ( 5 line pairs per $\mathrm{mm}$ ). Bottom panel: without PBS. Within group 2 and 3 groups 4 and 5 are displayed. The last resolved element is the first one in group 4 (16 line pairs per $\mathrm{mm}$ ).

of a focused USAF target (see Figure 2.15 for the setup) with and without the PBS. With the F/\# of the used optical setup the resolution is limited by the pixel size of the sensor. The pixel size is $48 \mu \mathrm{m}$, which means that a maximum of $\sim 10.4$ line pairs per $\mathrm{mm}$ can be resolved. The setup including the PBS has a magnification factor of $M=\frac{-f 2}{f_{1}}=-0.5$. Hence, the finest resolvable line pair is $\sim 5$ line pairs per $\mathrm{mm}$. In the top panel of Figure 2.17 we display the USAF target as seen by the $\mathrm{s}$ and $\mathrm{p}$ channels. The USAF element groups 2 and 3 are present. However, group 3 is not resolved. The last resolved line pair in both channels is group 2 element 3 , which corresponds to the expected 5 line pairs per $\mathrm{mm}$. We compare this image with an image taken before the PBS was introduced in the optical path. The magnification was 3 times higher, which means that 15 line pairs per mm should be resolved. As can be extracted from the bottom panel in Figure 2.17, the last resolved element is the first one in group 4, corresponding to the expected 16 line pairs per $\mathrm{mm}$. This implies that the PBS is not introducing any significant image aberrations.

Polarimetric data with the dual-beam configuration can be obtained by either demod- 
ulating both channels individually or by a simultaneous demodulation of both channels. The benefit for simultaneous demodulation is, that first order errors induced by seeing are removed completely, since both states are recorded at the same time (Donati et al. 1990; Semel et al. 1993; Skumanich et al. 1997; Bianda et al. 1998).

A brief academic example (based on Collados 1999) of how the use of a dual-beam configuration reduces residual cross-talk to first order in gain table errors is given. For an area of the Sun emitting the Stokes vector $I$, we have two areas on the sensor given by the two channels of the dual-beam configuration. In the first temporal modulation state, two intensities are recorded (see equation (2.31)):

$$
\begin{aligned}
& \hat{I}_{0}^{\mathrm{s}}=\sum_{i=0}^{3} O_{0 i}^{\mathrm{s}}\left(I_{i}+\delta I_{i}\right) \\
& \hat{I}_{0}^{\mathrm{p}}=(1+\delta g)\left(\sum_{i=0}^{3} O_{0 i}^{\mathrm{p}} I_{i}+\delta I_{i}\right),
\end{aligned}
$$

where $\delta I_{i}$ are the seeing induced errors and $\delta g$ is the uncertainty in the gain table. The gain table uncertainty is the pixel-to-pixel response differences, which are not corrected by applying a flat field. In the case of an ideal dual-beam system $O_{0 i}^{\mathrm{s}}=-O_{0 i}^{\mathrm{p}}$ for $i>0$. Let us simplify the problem by limiting the number of Stokes parameters to two and normalizing the modulation matrix $\left(O_{00}^{\{s, p\}}=1\right.$ and $\left.O_{01}^{\mathrm{s}}=1\right)$. Then

$$
\begin{aligned}
& \hat{I}_{0}^{\mathrm{s}}=I_{0}+\delta I_{0}+I_{1}+\delta I_{1} \\
& \hat{I}_{0}^{\mathrm{p}}=\left(I_{0}+\delta I_{0}-I_{1}-\delta I_{1}\right)(1+\delta g) .
\end{aligned}
$$

Given that solar light is only weakly polarized, i.e. $I_{0} \gg I_{1}$, the simultaneous demodulation of both channels leads to

$$
\begin{aligned}
& I^{\prime}=0.5 \cdot\left(\hat{I}_{0}^{\mathrm{s}}+\hat{I}_{0}^{\mathrm{p}}\right) \approx I_{0}+\delta I_{0}+\delta g\left(I_{0}+\delta I_{0}\right) \\
& I_{1}^{\prime}=0.5 \cdot\left(\hat{I}_{0}^{\mathrm{s}}-\hat{I}_{0}^{\mathrm{p}}\right)=I_{1}+\delta I_{1}-\delta g\left(I_{1}+\delta I_{1}\right)-\delta g\left(I_{0}+\delta I_{0}\right) .
\end{aligned}
$$

Therefore, the cross-talk $I_{0}=I$ to the measured $I_{i}^{\prime}$ for $i>0$ is removed. In the case of a balanced dual-beam setup, where $\delta g=0$, the measured Stokes parameters are only aberrated and cross-talk free.

However, the simultaneous dual-beam demodulation in practice suffers from flat field errors and also any misalignment of the two beams, even in the case of fast temporal modulation, i.e. $\delta I_{i} \approx 0$. Additionally, differential optical aberrations in both beams lead to spurious signatures. As an example, we show the Sr I data of Paper II in Figure 2.18, where we display the simultaneous dual-beam demodulated data in the last column.

Even after careful numerical alignment between the two dual-beam channels, small differences in the two channels add up dramatically in the polarization Stokes parameters. The quality of the Stokes $I$ image is, however, barely affected by the chosen demodulation method. This implies that the numerical alignment works well. Nevertheless, the spurious signals in the polarization Stokes parameters demonstrate that the alignment is not perfect. Especially as each polarimetric Stokes image is affected differently, a posteriori corrections would very difficult. We, therefore, chose to demodulate each channel individually and 


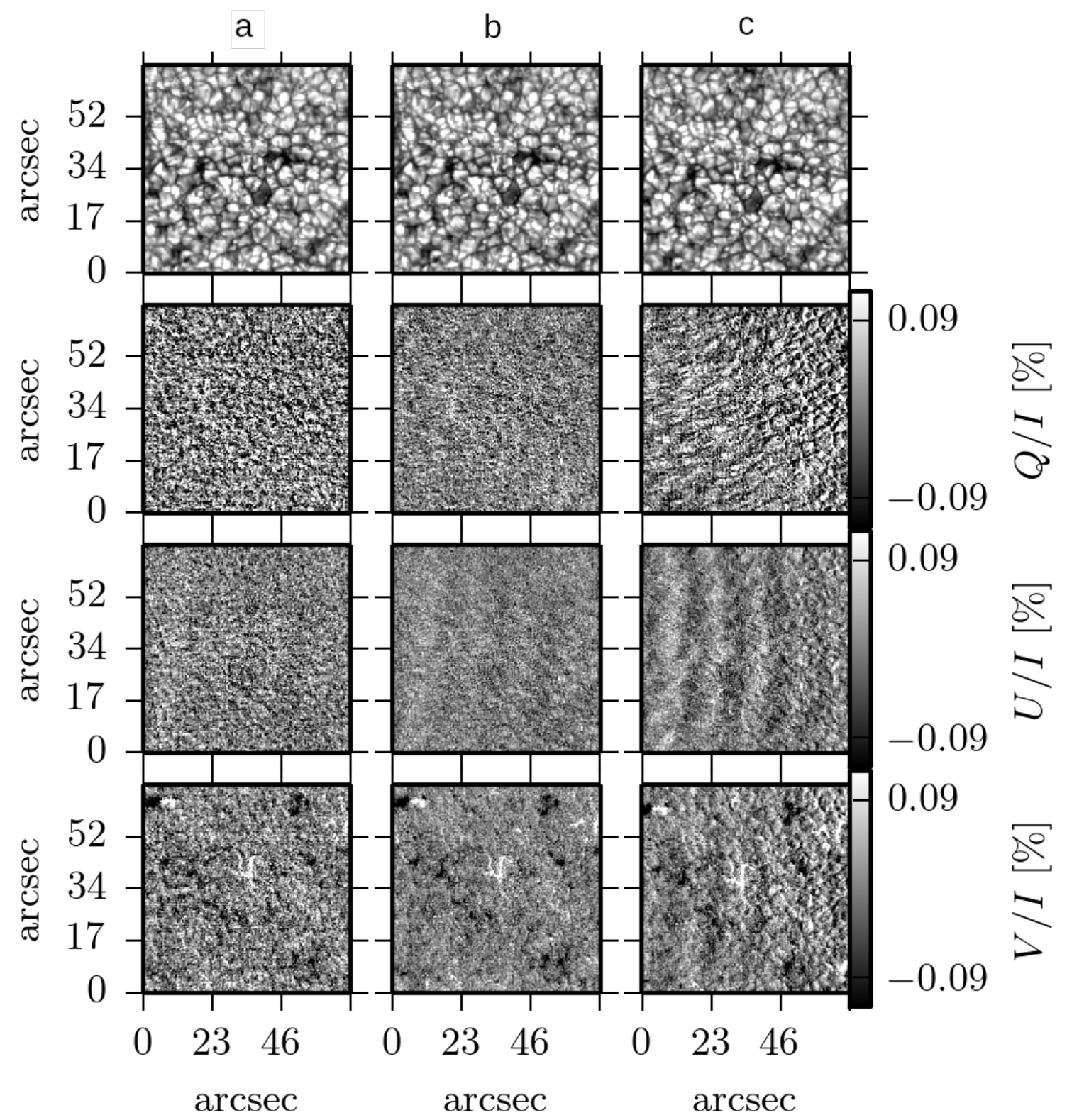

Figure 2.18: FSP 2 Stokes parameter images for different demodulation methods. (a) Single channel. (b) Individually demodulated and then combined channels. (c) Simultaneous dual-beam demodulated channels. The top panels correspond to the intensity, which appear identical, independently of the method.

combine the numerically aligned images to increase the signal-to-noise ratio, as shown in b) of Figure 2.18.

To conclude, the measurements have shown that the PBS meets our requirements, in particular in terms of the extinction ratio. Another relevant performance criterion is the differential error in the two channels, which we briefly investigated. We found that the setup does not allow for simultaneous dual-beam demodulation. Because of the high temporal modulation frequency of FSP 2, this is not a critical issue in reducing seeing induced errors. Further analysis of the residual differential effects between the PBS channels, and more 
elaborate mapping algorithms between the corresponding images may be required, in order to further improve the cross-talk suppression with respect to a single-beam configuration. However, by individually demodulating and then combining both dual-beam channels we are able to double the instrument's efficiency with respect to the single-beam configuration.

\subsubsection{Correction of intensity cross-talk}

In the following, we give an example of how the FSP 2 data in Paper II is corrected for Stokes $I$ cross-talk to $Q, U, V$.

Assume that the measured signal $I$ by a camera is a quadratic function of the incoming intensity $I_{S u n}$

$$
I=a I_{\text {Sun }}^{2}+b I_{\text {Sun }}+c,
$$

with the constant $c$ for residuals ${ }^{31}$ after the correction of the dark-current, $b$ the linear response (gain) of the camera and $a$ being the non-linear coefficient. Equation (2.36) models the simplest form of non-linear behaviour of a camera. Keller (1996) showed that a camera non-linearity $a$ combined with telescope polarization offset $\delta$ results in a cross-talk proportional to Stokes $I$ added to the normalized polarization image $(X / I)_{S \text { un }}$, where $X$ stands for any of $Q, U$ or $V$. Taking additionally into account the spatially distributed photon noise $r$, with $\operatorname{RMS}[r]=r,\langle r\rangle=0$, the measured Stokes $X / I$ is therefore: ${ }^{32}$

$$
X / I=(X / I)_{\text {Sun }}+\delta(1+(a / b) \cdot I)+r .
$$

None of the parameters are known a priori, except the measured intensity $I$. The goal is to estimate $(X / I)_{\text {corr }}=(X / I)_{S \text { un }}+r$ by using the measured Stokes $I$ image.

At disk center for quiet Sun regions, $\left\langle(X / I)_{S \text { un }}>=0\right.$ should hold. Thus, we take the spatial mean of equation (2.37):

$$
<X / I>=\delta+f \cdot<I>
$$

where we use the abbreviation $f=\delta \cdot(a / b)$, and consider that the polarimetric telescope offset is spatially independent, i.e. $\langle\delta\rangle=\delta$.

The first correction step is therefore to subtract $\langle X / I\rangle$ from $X / I$, which removes one unknown parameter:

$$
\begin{aligned}
& X / I-<X / I>= \\
& (X / I)_{\text {Sun }}+\delta+f \cdot I+r-\delta-f<I>= \\
& (X / I)_{\text {Sun }}+r+f(I-<I>)= \\
& (X / I)_{\text {corr }}+f(I-<I>) .
\end{aligned}
$$

Still, the factor $f$ needs to be determined. Recal that the goal is to estimate

$$
(X / I)_{\text {corr }}=X / I-<X / I>-f(I-<I>)=(X / I)_{S u n}+r,
$$

where all is known (measured) except $f$. Note that we are not interested to determine the coefficients $a$ and $b$ specifically. To estimate the unknown $f$, we subtract $f_{s}(I-<I>)$ for

\footnotetext{
${ }^{31}$ For example, stray-light of filtergraphs or spectrographs.

${ }^{32} \mathrm{We}$ assume that the linear and non-linear response are spatially constant for the sensor.
} 
systematic known $f_{s}$ values from $X / I-<X / I>$ in equation (2.39). Then, taking the RMS, we obtain:

$$
\begin{aligned}
& \operatorname{RMS}\left[X / I-<X / I>-f_{s}(I-<I>)\right]= \\
& \operatorname{RMS}\left[(X / I)_{\text {corr }}+f \cdot(I-<I>)-f_{s} \cdot(I-<I>)\right]= \\
& \operatorname{RMS}\left[(X / I)_{\text {corr }}+\left(f-f_{s}\right) \cdot(I-<I>)\right] .
\end{aligned}
$$

The left hand side of equation (2.41) has a global minimum at $f=f_{s}$. Thereby, the minimum of $R M S\left[X / I-<X / I>-f_{s}(I-<I>)\right]=R M S\left[X / I^{\prime}\right]$ with respect to $f_{s}$ determines the factor $f$. We plot the $R M S\left[X / I^{\prime}\right]\left(f_{s}\right)$ for both dual-beam channels (denoted by $\mathrm{s}$ and $\mathrm{p}$ ) for a range of factors $f_{s}$ for all images in the time series for the Sr I data of Paper II.
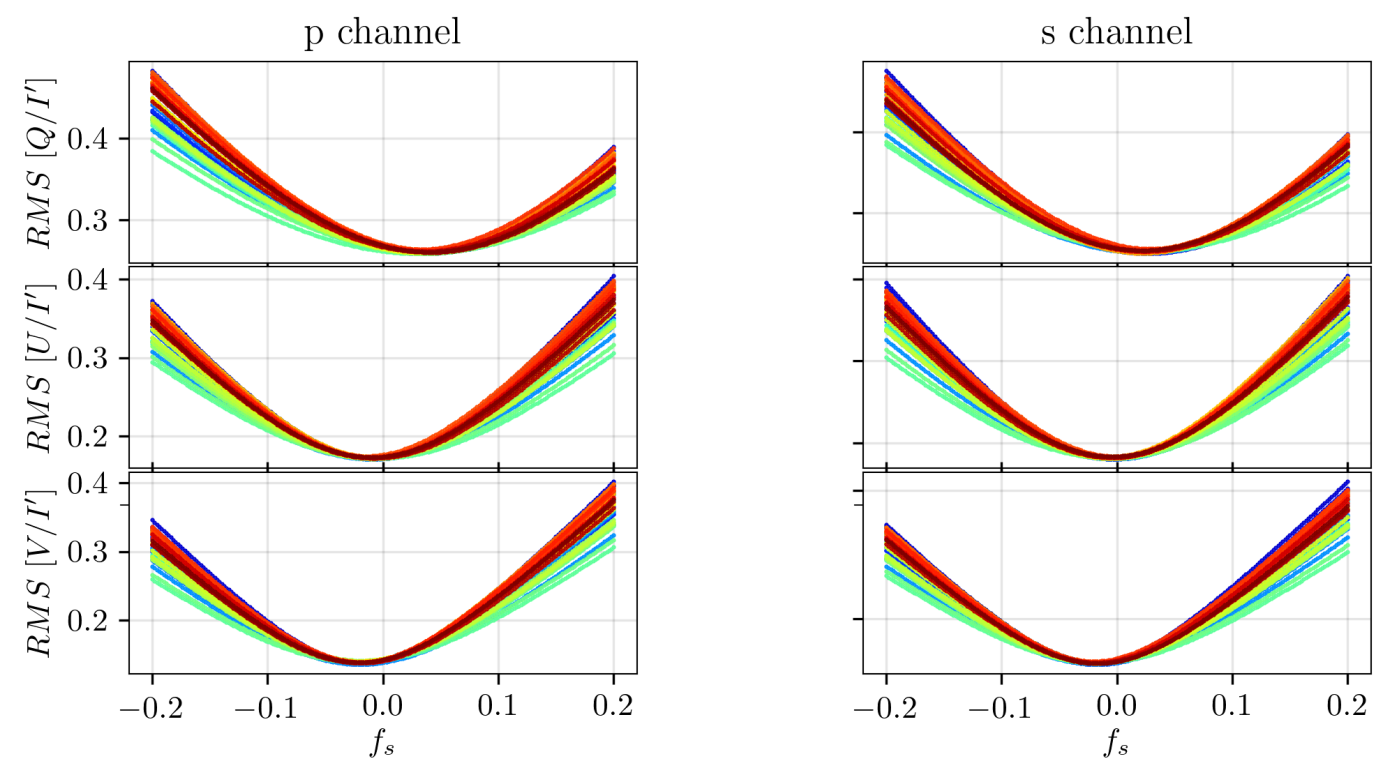

Figure 2.19: Standard deviation of $X / I^{\prime}$ as a function of factors $f_{s}$ to determine the factor $f$ to correct Stokes $I$ cross-talk, for both dual-beam channels of FSP 2 separately. We determine the standard deviation for each image in the time series, which is represented by the array of curves per Stokes parameter. The colour ranges from blue to red, corresponding to the time when the image was taken, i.e. blue is the first image and red the last.

We find that, in general, the largest factors $f$, where $f$ characterizes $\min \left(R M S\left[X / I^{\prime}\right]\left(f_{s}\right)\right)$ $=R M S\left[X / I^{\prime}\right](f)$, are found for $Q / I$. For $U / I$ they are smaller, therefore $U / I$ is barely affected by Stokes $I$ cross-talk. This is also reflected by an almost negligible polarimetric offset in Stokes $U / I$, while the mean of the uncorrected Stokes $Q / I$ is $2 \%$. Note also that the optimum factors $f$ which minimize the $R M S\left[X / I^{\prime}\right]$ for each image of the time series are very similar, but not identical. For example, for the $Q / I$ in the s-channel the mean of $f$ of the time series is $0.035 \%$ counts $^{-1}$ with a standard deviation of $0.003 \%$ counts $^{-1}$ (the step size for $f_{s}$ is $10^{-3} \%$ counts $^{-1}$ ). Just correcting the temporally averaged polarimetric images with a temporally averaged intensity image will therefore leave residual Stokes $I$ cross-talk at a level of $0.1 \%$. This is expected, as the observing conditions may change fast. Therefore, high-cadence data can be corrected easier for Stokes $I$ cross-talk than low-cadence data. 
As an example for a successful Stokes $I$ cross-talk removal, we plot the uncorrected, i.e. originally observed, Stokes $Q / I$ in the right panel of Figure 2.20, which shows pronounced cross-talk from Stokes $I$ (shown at the left). In the center of Figure 2.20, we plot $(Q / I)_{\text {corr }}=Q / I-<Q / I>-f \cdot(I-<I>)$ for one image in the time series, with $f=0.037 \%$ counts $^{-1}$. It is important to note that this method may remove spatial structures introduced by Stokes $I$, but fails to remove any additional noise introduced by the same. This is because the noise is already present in the originally measured Stokes parameter $X / I{ }^{33}$ Thereby, the noise level of Stokes $I$ cross-talk affected polarimetric data might be higher compared to cross-talk free polarimetric data. For the FSP 2 data, we find that Stokes $U / I$ has a significantly lower noise level than Stokes $Q / I$, although the polarimetric efficiencies are comparable.
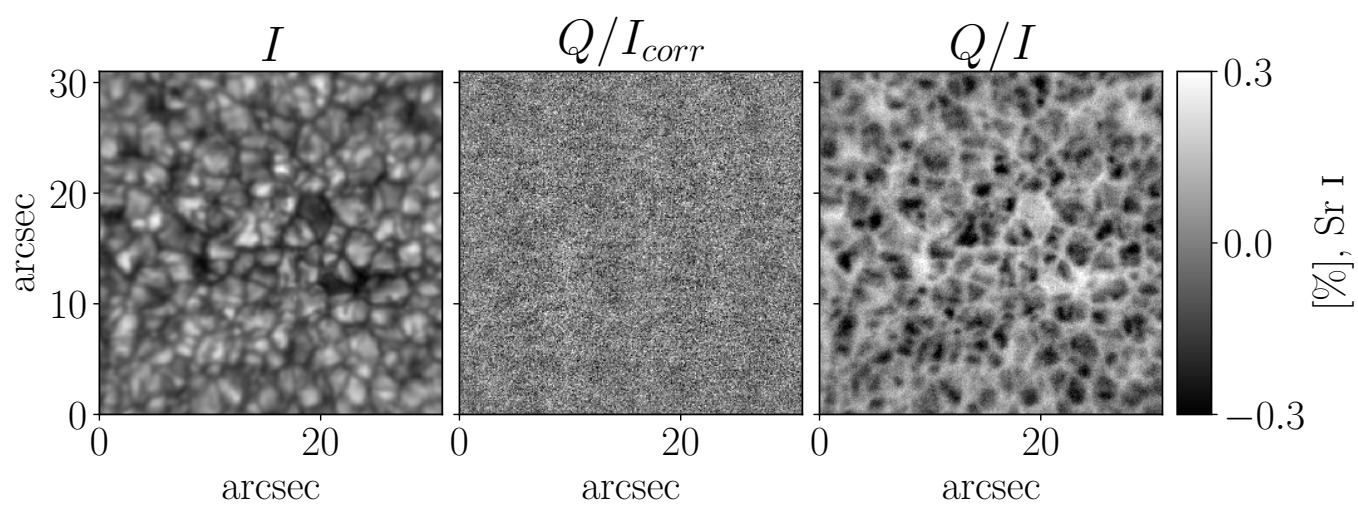

Figure 2.20: Continuum intensity image (left), corrected and uncorrected Stokes $Q / I$ (center and right) for a single time snapshot in the Sr I data presented in Paper II.

By using equation (2.38) it is possible to estimate the telescope offset for $Q / I$ with the values given by our measurement $(<Q / I\rangle=2 \%,\langle I\rangle=31.6$ counts and $f=0.03 \%$ counts $^{-1}$ for one example image in the time series):

$$
\delta=f \cdot\langle I>-<Q / I>\approx 1.1 \% .
$$

This is a relatively low value for telescope polarization. The used telescope for the data in Paper II was the Dunn Solar Telescope (DST) in Sunspot, New Mexico. It has been tried a few times to determine a time (and wavelength) dependent model of the telescope Mueller matrix (Skumanich et al. 1997; Socas-Navarro et al. 2011; Derks et al. 2018), which turns out to be difficult for the DST. However, different models vary in the absolute values for the telescope matrix elements, but are consistent with a low intensity to linear polarization cross-talk (well below 5\%) in the morning, i.e. between 14 UTC and 15 UTC, at the time of the observation and at a wavelength of about $459 \mathrm{~nm}$ (observed in March, while we observed in August, see Figure 12 of Derks et al. 2018).

\subsubsection{Fabry-Pérot interferometer based wavelength discrimination}

A Fabry-Pérot etalon (FP) is an optical component consisting of two highly reflecting parallel (coated) glass plates, separated by an air-gap of a certain distance, forming a cavity.

\footnotetext{
${ }^{33}$ We use the terms Stokes, Stokes parameter, and normalized Stokes parameter interchangeably.
} 
The resonant cavity produces a periodically varying transmission as a function of the wavelength. The varying transmission is due to the interference of multiple reflections of light in the cavity, where each reflected light ray passing the cavity accumulates a phase. If the cavity length is a multiple of the wavelength, the multiple reflected light rays interfere constructively and are transmitted with high probability. The principle of wavelength discrimination by a FP is therefore based on interferometry. FP's are tuneable by changing the cavity length.

The transmission function $I_{t}$ of a FP filled with air under normal incidence of the intensity $I_{0}$ is given by (Born \& Wolf 1970):

$$
I_{t}(\Delta)=\frac{I_{0}}{1+F \sin ^{2}(\Delta / 2)},
$$

where

$$
F=\frac{\pi \sqrt{R}}{1-R}
$$

and

$$
\Delta=\frac{4 \pi L}{\lambda}
$$

$F, R$, and $L$ are the finesse coefficient, the reflectivity, and the cavity length, respectively. The two parameters characterizing a FP are: The free spectral range (FSR) and the finesse. Combining etalons extents the free spectral range. The FSR is the wavelength difference between adjacent transmission peaks in the FP spectra and is wavelength dependent:

$$
\mathrm{FSR}=\frac{\lambda^{2}}{2 L}
$$

The finesse is the ratio between the FSR and the $F W H M^{34}$ of the transmission peaks. High finesse is achieved with high reflectivity of the glass plates. For FPs used in solar physics, the reflectivity is usually $90 \%$ or better. Defects on the glass surface decrease the finesse by spectral broadening of the transmission profile. Two examples of normalized transmission profiles with different cavity reflectivities (and therefore different finesse) as a function of the wavelength are plotted in Figure 2.21. The black profile with high reflectivity has narrower peaks than the blue profile with lower finesse.

A collimated setup suffers from a field-dependent blue-shift of the FP bandpass, which usually has to be calibrated. To avoid this problem, we restricted our field of view in Paper II to a confined region close to the center of the FP. To reduce the contribution of secondary peaks usually a pre-filter is placed in front of the FP. The resulting spectral transmission profile from a FP and prefilter is calculated by convolving both transmission profiles.

\footnotetext{
${ }^{34}$ Full-width at half-maximum.
} 


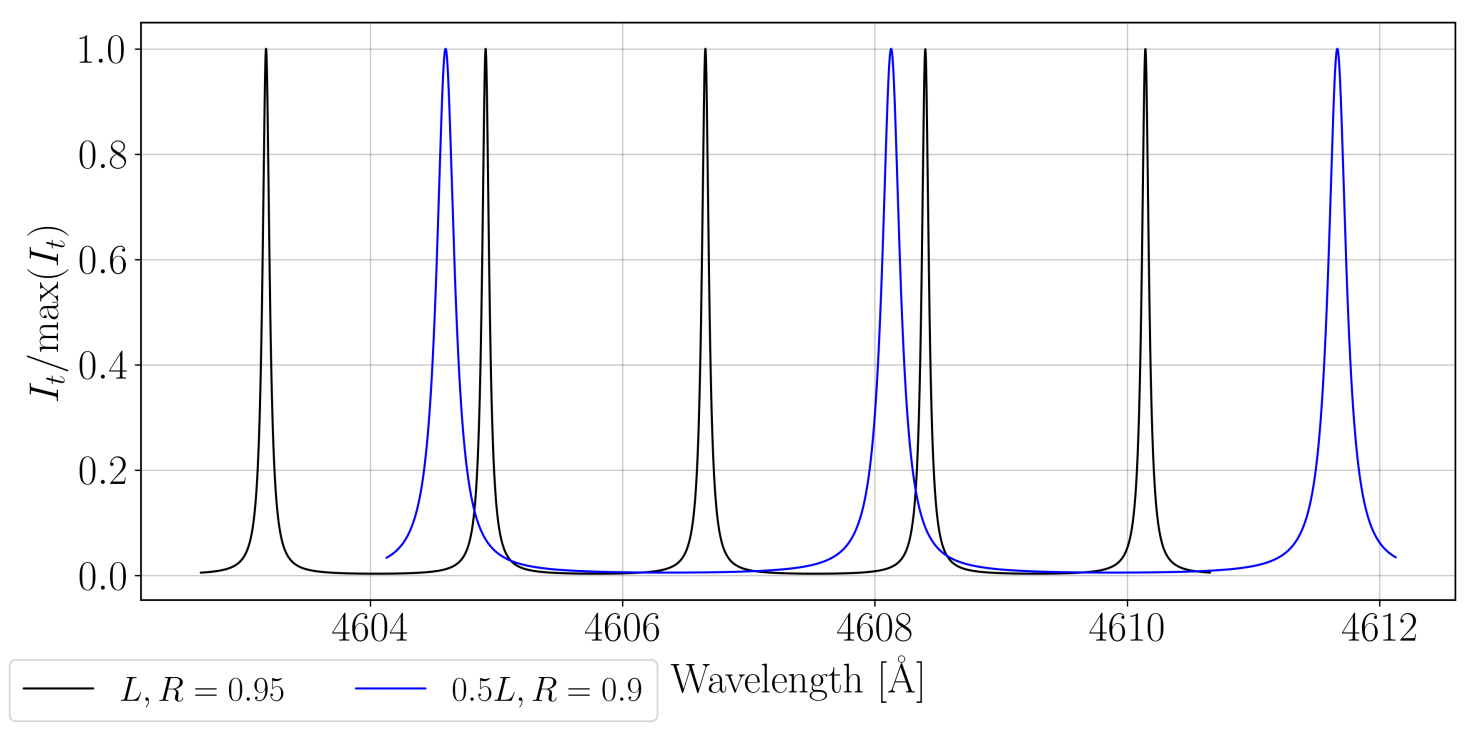

Figure 2.21: Two normalized transmission profiles for FP etalons with different reflectivities and cavity lengths $(L=6.09 \mathrm{~cm}$ ). The finesse for the black profile is 26.1 (high reflectivity $R$ ), while for the blue profile it is 20.7 (low reflectivity $R$ ). The FSR is larger for smaller cavity lengths, i.e. the distance between the peaks of the black profile is smaller than for the blue profile, calculated with half the cavity length. 


\section{Paper I: Detection of spatially structured scattering polarization of Sr I 4607.3 A with the Fast Solar Polarimeter}

published on 22.11.2018 in

Astronomy \& Astrophysics, 619, A179 ${ }^{1}$

Franziska Zeuner, Alex Feller, Francisco A. Iglesias \& Sami K. Solanki

My contribution: Reduction and analysis of the data, and writing of the manuscript.

\subsection{Abstract}

Scattering polarization in the $\mathrm{Sr}_{\mathrm{I}} 4607.3 \AA$ line observed with high resolution is an important diagnostic of the Sun's atmosphere and magnetism at small spatial scales. Investigating the scattering polarization altered by the Hanle effect is key to constraining the role of small-scale magnetic activity in solar atmospheric activity and energy balance. At present, spatially resolved observations of this diagnostic are rare and have not been reported as close to the disk center as for $\mu=0.6$.

Our aim is to measure the scattering polarization in the $\mathrm{Sr}$ I line at $\mu=0.6$ and to identify the spatial fluctuations with a statistical approach.

Using the Fast Solar Polarimeter (FSP) mounted on the TESOS filtergraph at the German Vacuum Tower Telescope (VTT) in Tenerife, Spain, we measured both the spatially resolved full Stokes parameters of the Sr I line at $\mu=0.6$ and the center-to-limb variation of the spatially averaged Stokes parameters.

We find that the center-to-limb variation of the scattering polarization in the Sr I line measured with FSP is consistent with previous measurements. A statistical analysis of Stokes $Q / I$ (i.e., the linear polarization component parallel to the solar limb), sampled with $0.16^{\prime \prime}$ pixel $^{-1}$ in the line core of Sr I reveals that the signal strength is inversely correlated with the intensity in the continuum. We find stronger linear polarimetric signals corresponding to dark areas in the Stokes $I$ continuum image (intergranular lanes). In contrast, independent measurements at $\mu=0.3$ show a positive correlation of $Q / I$ with

1 (C) ESO. Reproduced with permission. https: //doi .org/10.1051/0004-6361/201833241 
respect to the continuum intensity. We estimate that the subregion diameter responsible for the excess $Q / I$ signal is on the order of $0.5^{\prime \prime}-1^{\prime \prime}$.

The presented observations and the statistical analysis of $Q / I$ signals at $\mu=0.6$ complement reported scattering polarization observations as well as simulations. The FSP has proven to be a suitable instrument to measure spatially resolved scattering polarization signals. In the future, a systematic center-to-limb series of observations with subgranular spatial resolution and increased polarimetric sensitivity $\left(<10^{-3}\right)$ compared to that in the present study is needed in order to investigate the change in trend with $\mu$ that the comparison of our results with the literature suggests.

\subsection{Introduction}

The quiet solar photosphere is thought to be filled by small-scale and weak magnetic fields, as predicted by small-scale dynamo simulations of Vögler \& Schüssler (2007) and Rempel (2014). To test this model, detailed measurements of the often weak magnetic field and its spatial distribution is required. However, observations draw an inhomogeneous picture on magnetic field strength, inclination and distribution, see Lagg et al. (2016), Stenflo (2011) and reviews on this topic by de Wijn et al. (2009), Steiner \& Rezaei (2012) and Borrero et al. (2015). Observationally determining properties of fields weaker than a few hundred Gauss, which are tangled at spatial scales close to the observations' spatial resolution is quite a challenge, as the Zeeman effect as a diagnostic tool has two major intrinsic disadvantages for extracting information about these fields. Firstly, the sensitivity to weak magnetic fields is low, particularly to the component perpendicular to the line-of-sight, and secondly, if the magnetic field is turbulent, signal cancellation within a resolution element is possible. This makes it particularly difficult to probe the magnetic field's properties in inter-network regions, as the results conspicuously depend on the method and spectral line employed, see e.g., Borrero et al. (2015). To a large extent, these disadvantages are overcome by employing the Hanle effect, see for example, Landi Degl'Innocenti \& Landolfi (2004) and Stenflo (1994).

A photospheric line widely used for Hanle diagnostics is Sr I $4607.3 \AA$, which has the advantage of providing scattering signals at $\mu=0.1$ above $1 \%$ (see, e.g., Stenflo et al. 1997 and Gandorfer 2002). Here $\mu=\cos (\theta)$, where $\theta$ is the heliocentric angle and $\mu=0$ at the solar limb. So far, measurements with the required polarimetric sensitivity of the scattering polarization signals in this line suffer from insufficient spatio-temporal resolution (see Trujillo Bueno \& Shchukina 2007 and references therein). Additionally, the observed target needs to be sufficiently far from the solar limb to confidently correlate the granulation pattern with the detected polarimetric signals for interpretation. Observations with a spatial resolution of about $0.6^{\prime \prime}$ by Malherbe et al. (2007) at $\mu=0.3$ from the west limb display a positive correlation between the Stokes $Q / I$ signal in the Sr I line core and continuum intensity. Their findings possibly hint at a Hanle effect acting in the intergranular regions where higher magnetic fields are expected, in agreement with simulations carried out by Trujillo Bueno et al. (2004). However, close to the disk center, Trujillo Bueno \& Shchukina (2007) predicted theoretically that most of the scattering polarization is produced by local symmetry breaking of the radiation field by atmospheric inhomogeneities. In this case, the theoretically expected $Q / I$ signal fluctuations lie between $-0.08 \%$ and $0.9 \%$, as they would be observed with a $1 \mathrm{~m}$ telescope and a spectral resolution of $25 \mathrm{~m} \AA$ at $\mu=0.5$, without 
considering a depolarizing magnetic field.

Here we present the results of two sets of observations carried out at the German Vacuum Tower Telescope (Tenerife, Spain) in the Sr I $4607.3 \AA$ line taken with the Fast Solar Polarimeter (Iglesias et al. 2016) attached to the TESOS filtergraph (Kentischer et al. 1998; Tritschler et al. 2002). First we check if the data obtained with the Fast Solar Polarimeter are consistent with those in the literature by measuring the center-to-limb variation of the linear polarization in $\mathrm{Sr}$ I and in a neighboring $\mathrm{Fe}_{\mathrm{I}}$ line that does not show scattering polarization. This center-to-limb variation of the $Q / I$ amplitude is compared with published results. In a second step, we use a statistical approach to analyze photon-noise limited spatial fluctuations of the $Q / I$ signal at subgranular resolution and (1) search for a correlation between the $Q / I$ amplitude in the line core and continuum intensity and (2) estimate the structure size of scattering polarization signals.

\subsection{Observations and data reduction}

Our observations were obtained with a prototype of the Fast Solar Polarimeter (FSP) mounted at the Vacuum Tower Telescope (VTT) at the El Teide observatory on Tenerife, using the TESOS Fabry-Pérot tunable filtergraph. This particular prototype of FSP is composed of a fast, cooled pnCCD camera synchronized with a ferro-electric liquid crystal based polarization modulator. The image dimensions are $248 \times 256 \mathrm{pixel}^{2}$. The sampling of $0.08^{\prime \prime}$ pixel $^{-1}$ corresponds to approximately critical sampling at the diffraction limit of the $0.68 \mathrm{~m}$ VTT aperture. The TESOS bandpass has a FWHM of about $25 \mathrm{~m} \AA$ (Beck et al. 2010). FSP is run with a polarization modulation frequency of $100 \mathrm{~Hz}$, which corresponds to a frame rate of 400 frames s$^{-1}$ (four modulation states per cycle). For more details of the FSP and the used modulation scheme see Iglesias et al. (2016). Calibration data, that is, dark images, a modulated flat field for all wavelength positions (moving the telescope randomly around disk center) and polarimetric calibration images were recorded within a few minutes of the observations.

\subsubsection{Center-to-limb variation of scattering polarization}

\subsubsection{Observations}

On 6 May 2015 we observed quiet Sun regions at different limb distances between 17 and 19 UTC without using the adaptive optics (AO) system. We recorded the full Stokes vector at 11 wavelength positions, whereby the full record of all positions is called a scan: $[-90,90,-60,60,-40,40,-20,20,-10,10,0] \mathrm{m} \AA$ around a central wavelength. As central wavelengths we used $\lambda_{c, \mathrm{~S} r}=4607.33 \AA$ centered on the core of the Sr I line and $\lambda_{c, \mathrm{Fe}}=4607.63 \AA$ centered on the core of a neighboring Fe I line. Ten scans through the Sr I line were made, each at a different solar disk position, ranging between solar disk center and the solar north limb in steps of $\Delta \mu=0.1$. In this way we do not need to correct for Doppler shifts due to solar rotation. The Fe I line was scanned at $\mu=[1,0.8,0.27,0.1]$. At $\mu=0.1$ the solar limb is visible as a strong intensity drop in the upper part of the images. The individual scans took between $99 \mathrm{~s}$ and $106 \mathrm{~s}$ to complete and will be referred to as scan data hereinafter. 


\subsubsection{Data reduction}

For a detailed description of the basic reduction steps, see Iglesias et al. (2016). The basic data reduction consists of three steps. They correct for offsets by subtracting a low-noise dark frame, image smearing, and common mode. The common mode signal is estimated from shielded pixels and subtracted from each semi-row. The image smearing, caused by shutter-free operation of frame-transfer CCD detectors, is corrected by using a modified standard model developed and implemented by Iglesias et al. (2015). After the basic reduction, 800 frames within each modulation state and wavelength position are averaged to a single image. The flat field and the scan data are corrected and averaged identically.

For polarimetric calibration, FSP recorded 19 predetermined polarization states, generated in front of the modulator. Therefore, instrumental polarization caused by optical elements of the telescope located in front of the modulator is excluded from the calibration. The measured intensities were used to fit the elements of the over-determined $4 \times 4$ (de-)modulation matrix. The demodulation matrix was then applied to the flat field and the scan data in the same way. The polarimetric calibration is described in detail by Iglesias et al. (2016).

The flat field and scan Stokes $Q, U, V$ images were normalized to Stokes $I$. The flat field $Q / I, U / I$ and $V / I$ images were then subtracted from the respective scan data. This procedure was chosen to take into account artificial offsets in $Q / I, U / I$ and $V / I$ due to telescope polarization and to remove polarized fringes. As only very low polarization is expected in the continuum (Gandorfer 2002), we calculated spatial averages of the $Q / I$, $U / I$ and $V / I$ images taken at $-90 \mathrm{~m} \AA$ and subtracted them as an offset from all wavelength points and corresponding polarization state images. A heuristic cross-talk removal was applied to correct the scan data for the uncalibrated telescope polarization, since there is no reliable telescope model available for the used wavelength range. We corrected for cross-talk between $V / I$ and $Q / I$, as well as between $V / I$ and $U / I$. This was done by rotating the whole FOV around the $U$ and $Q$ axes of the Poincare sphere until the $V / I$ signal was minimized. The estimated cross-talk with this method is $(9 \pm 2) \%$ for $V / I$ to $Q / I$, corrected by a $5^{\circ}$ rotation around the $U$ axis. For the cross-talk from $V / I$ to $U / I$ we estimated $(57 \pm 1) \%$, corrected with a $35^{\circ}$ rotation around the $Q$ axis. The direction of $+Q / I$ is parallel to the north solar limb.

We then averaged all pixels for a given $\mu$ value and for one wavelength. Given that $\mu$ changes more rapidly over a given distance on an image with smaller $\mu$-values, the image closest to the limb was divided into equally sized stripes of width $1.2^{\prime \prime}$ that are parallel to the solar limb and the linear polarization within these stripes was averaged. The need for such narrow stripes is heightened by the fact that the $Q / I$ amplitude also increases most rapidly close to the limb. The solar limb was defined as the part of the image where the slope of the intensity was steepest. Since the AO system was off, the limb position changed in the field of view per wavelength position and was therefore obtained for each wavelength position separately.

\subsubsection{Spatially resolved Stokes measurements}

\subsubsection{Observations}

Our spatially resolved Stokes measurements in the Sr I line were performed on May 27 2014 between 11 and 13 UTC. The AO was locked on a quiet Sun region at $\mu=0.6$ 
toward the north solar limb. Our FOV is $20^{\prime \prime}$ by $20^{\prime \prime}$. Five wavelength positions relative to $4607.3 \AA([-90,-60,30,-30,0] \mathrm{m} \AA)$ were scanned. The exposure time was $1.25 \mathrm{~s}$ per modulation state and wavelength position for a single such scan, resulting in a total exposure time of $5 \mathrm{~s}$ per wavelength position. A single scan over all wavelengths required $32 \mathrm{~s}$, implying a duty cycle of about $78 \%$. In total, ten such scans were obtained.

\subsubsection{Data reduction}

The basic data reduction, the demodulation and normalization of the Stokes images were done as described in Sect. 3.3.1.2, including the flat-fielding procedure. Because of this procedure, which requires demodulation before flat-fielding, we were not able to make use of image restoration techniques to further increase the spatial resolution in the polarization Stokes images. From the demodulation matrix, we obtain $95 \%$ total polarimetric efficiency (del Toro Iniesta 2004). For each scan all frames at a given wavelength position and modulation state were averaged.

We determined the orientation of our Stokes reference system with respect to the solar limb by using a dataset acquired at 13 UTC in the Fe $\mathrm{I} 4607.6 \AA$ line, where we observed a pore region. We rotated our pore images by $10^{\circ}$ anti-clockwise until they matched SDO/HMI Stokes images of the same region in both orientation and polarization, which for HMI are calibrated to have Stokes $+Q / I$ parallel to the north limb (Schou et al. 2012). We heuristically corrected for cross-talk between $V / I$ and $Q / I$, and $V / I$ and $U / I$ by minimizing a small longitudinal Zeeman signature at the edge of our FOV in $U / I$ and $Q / I$, using a Poincaré rotation, as in Sect. 3.3.1.2. The used angles were $17^{\circ}$ and $41^{\circ}$ for rotation around the $Q$ axis and the $U$ axis, respectively, corresponding to cross-talk values of $(29 \pm 2) \%$ between $Q / I$ and $V / I$ and $(75 \pm 1) \%$ between $U / I$ and $V / I$. For further analysis of the scattering polarization, regions with a Zeeman signature were excluded (see Figure 3.1), which resulted in a region of interest of $8^{\prime \prime}$ by $17^{\prime \prime}$. Our data suffered from variable seeing conditions. For further analysis we therefore selected the first two scans with stable AO locking. To increase the signal-to-noise ratio in the polarization signal while not degrading spatial resolution significantly, we averaged both scans and applied a $2 \times 2$ spatial binning. Consequently, in the following we analyze one image per Stokes parameter and per wavelength position, where each image has a sampling of $0.16^{\prime \prime}$ pixel $^{-1}$ and $2.5 \mathrm{~s}$ exposure time. 

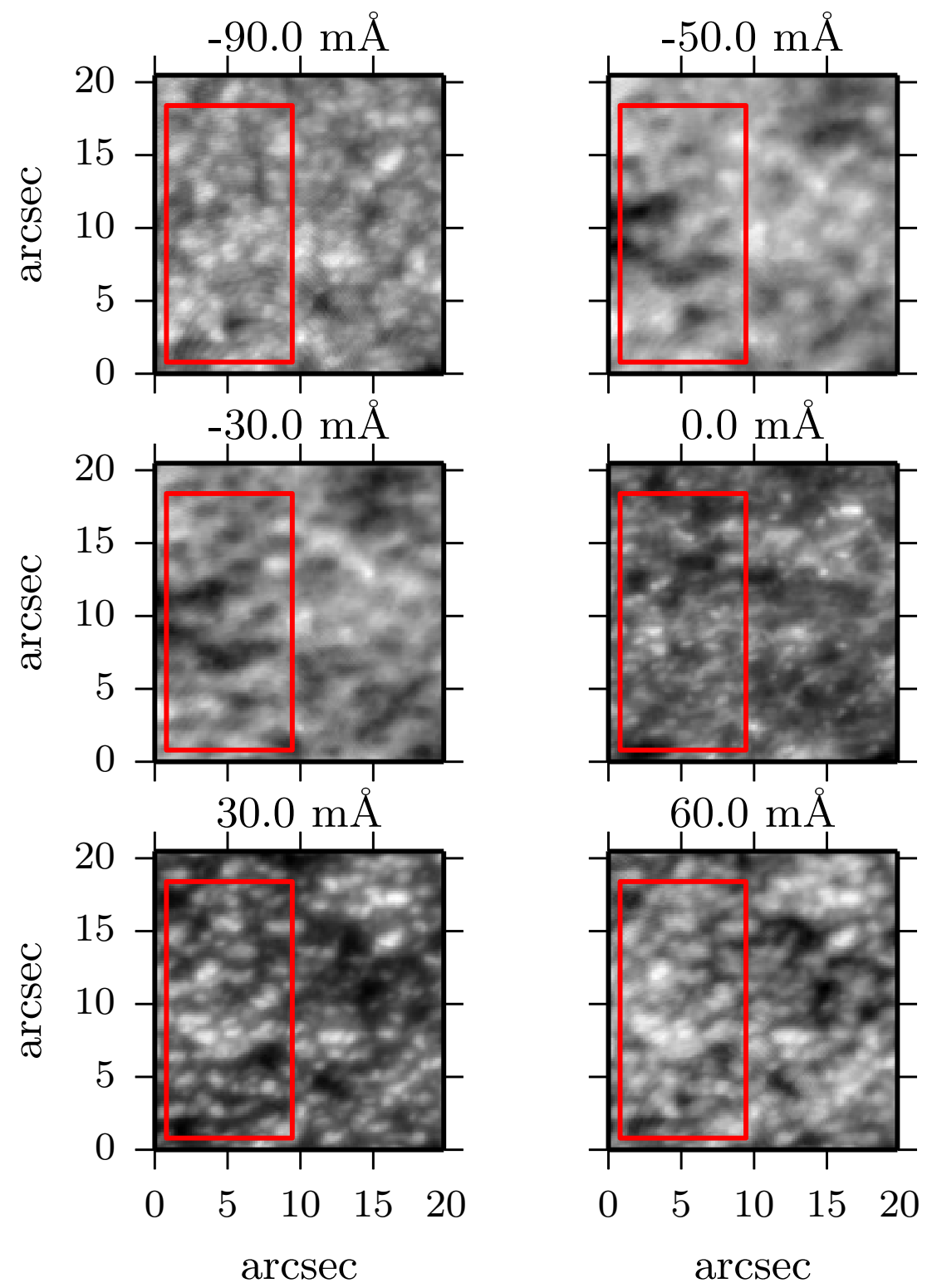

Figure 3.1: Stokes $I$ images for different wavelengths at $\mu=0.6$. The region of interest for the analysis lies inside the red box, which excludes Zeeman signatures found in the right corner. The north solar limb is parallel to the shorter axis of the red box. The image labeled with $30 \mathrm{~m} \AA$ corresponds to the Sr I line core.

\subsection{Results}

\subsubsection{Center-to-limb variation of scattering polarization}

Spatially averaged Sr I Stokes profiles for $\mu=1.0,0.6,0.1$ are plotted in Figure 3.2. Each data point in the polarimetric spectral profiles has a low photon noise level (i.e., $\sigma I /\langle I\rangle<$ $0.02 \%$ ) due to the spatial averaging. For Stokes $I$, we additionally plot Fourier Transform Spectrograph (FTS) atlas data by Neckel (1999) and the Stokes $I_{\text {Gan02 }}$ close to the limb 


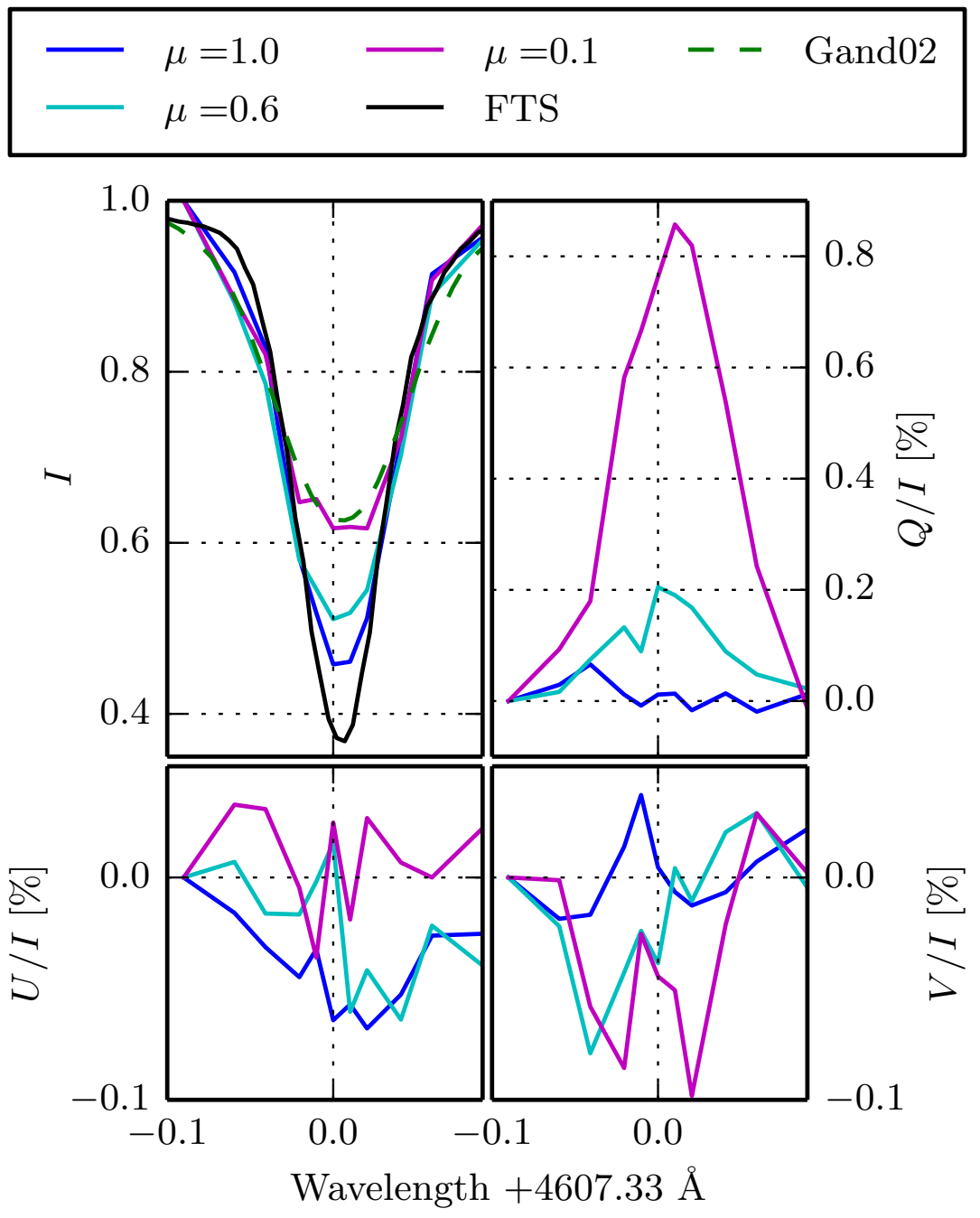

Figure 3.2: Center-to-limb variation of the polarized profiles of the $\mathrm{Sr}$ I $4607.3 \AA$ line, spatially averaged over one image per $\mu$-value. To avoid cluttering, only three out of the ten measured $\mu$ positions are displayed. For comparison, the Stokes $I$ profile given in the FTS atlas (black solid line) and Stokes $I_{\text {Gan } 02}$ taken from Gandorfer (2002) (green dashed line) is included. Each Stokes $I$ spectrum is normalized to the continuum point at $-90 \mathrm{~m} \AA$. Residual polarimetric offsets after flat-fielding are removed by subtracting the value of $Q / I$, $U / I$ and $V / I$ at $-90 \mathrm{~m} \AA$ from the respective profiles at each wavelength position.

at $\mu=0.1$ based on the atlas of Gandorfer (2002), which has been provided in electronic form by IRSOL as a compilation by Stenflo (2014). The line depth of the Stokes $I_{\text {Gan } 02}$ and the limb measurement are comparable, but for the FTS and our measured line at disk center there is a line depth difference of about $10 \%$. This is likely due to spectral scattered light as well as lower spectral resolution of TESOS compared to the FTS. While Stokes $U / I$ and $V / I$ show no systematic correlation with limb distance within the spectral width of the scan in Figure 3.2, the Stokes $Q / I$ peak signal systematically increases with lower $\mu$ values, as expected.

We fitted a Gaussian function to the $Q / I$ spectra for each $\mu$ position separately, and plot amplitudes with respect to $\mu$ in Figure 3.3. For optimum fitting results the free 


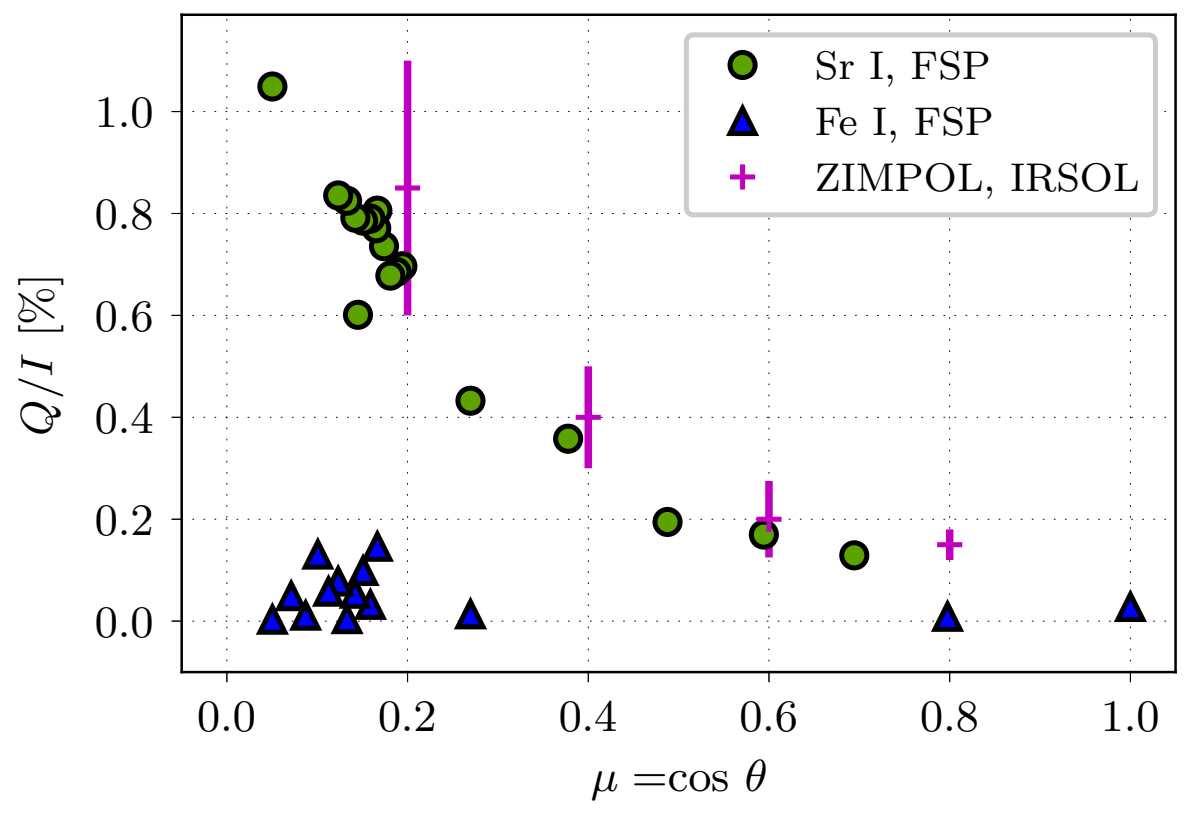

Figure 3.3: Stokes $Q / I$ amplitude as a function of limb distance $\mu=\cos (\theta)$. The $\operatorname{Sr}$ I data points represent the amplitude of a Gaussian fit to the $Q / I$ line, while the Fe I data points are spectrally and spatially averaged values. We note that the scale is linear and that below $\mu=0.2$ less pixels were averaged for each data point. The signal-to-noise ratio beyond $\mu=0.7$ is below two and therefore the fitted amplitudes are discarded. For comparison, a range of Sr i polarization values obtained by ZIMPOL and a stationary IRSOL polarimeter (magenta bars show the range of values) are taken from Stenflo et al. (1997).

parameters were not constrained. The standard deviation of $0.03 \%$ for $Q / I, U / I$ and $V / I$ in the spectral profile at $\mu=1$ is taken to be an estimation of the noise level as no signal is expected. Amplitudes were discarded if their ratio with the aforementioned noise level were smaller than two. The low contrast of the intensity images $(<5 \%)$ justifies the use of a constant weighting factor corresponding to the mean photon noise. The $95 \%$ confidence interval for the amplitude is $0.2 \%$. We find a maximum $Q / I$ amplitude at $\mu=0.05$ of more than $1 \%$, which is in agreement with observations summarized by Malherbe et al. (2007). Values in the literature of the $Q / I$ amplitude at this $\mu$ value range from $0.95 \%$ (Faurobert et al. 2001) to 1.5\% (Stenflo \& Keller 1997), which encompass our measured $Q / I$ amplitude. In Figure 3.3 we compare our $Q / I$ amplitudes for $\mu=0.2,0.4$ and 0.8 with a range of values obtained by Stenflo et al. (1997) with ZIMPOL and a stationary polarimeter installed at IRSOL. Our amplitudes at these $\mu$ values are again consistent with the measurements in the literature. Additionally, in Figure 3.3, we present as a blind test the $Q / I$ polarization we found in the neighboring Fe I $4607.6 \AA$, averaged over the spectral width of the measurement, because the Stokes $Q / I$ is flat. As Fe I is insensitive to scattering polarization, the polarization signals do not depend on the limb distance. For $\mu<0.2$ we spatially averaged across a smaller spatial region. This results in a higher noise level, which explains the higher scattering of the Fe I data points below $\mu=0.2$. 


\subsubsection{Spatially resolved Stokes measurements}

The Stokes $I$ images at each recorded wavelength position in the quiet Sun observation at $\mu=0.6$ are displayed in Figure 3.1. Based on the continuum Stokes $I$ images at $-90 \mathrm{~m} \AA$, we obtained a granulation intensity contrast of $2.5 \%$. To obtain the noise level for Stokes $Q / I$ per pixel the spatial power spectrum of the associated image is calculated. Based on the diffraction limit of the telescope, the cut-off frequency was computed. The power contained at spatial frequencies beyond the cut-off was assumed to be representative for the photon noise level. We obtained a noise level of $0.3 \%$ per pixel for Stokes $Q / I$, which is consistent with the photon noise level estimated from Stokes $I$ images. We note that these images were averaged over two scans and spatially binned as described in Sect. 3.3.2.2. At this noise level of $0.3 \%$, we do not find any direct visual evidence for spatial signal fluctuations at sub-granular scales in any polarization state.

The amount of the line core polarization signal in Stokes $Q / I$ is close to $0 \%$ in the granules, while for intergranules the values are around $0.25 \%$. The spatial mean of Stokes $Q / I$ in the line core is $0.1 \%$, which is lower than what is shown in Figure 3.2 for $\mu=0.6$ but close to the lower edge of the values found by Stenflo et al. (1997). The origin of this discrepancy is unknown, but possible spatial fluctuations should be unaffected by a shift of the mean signal.

\subsubsection{Correlations with the continuum intensity}

To reveal correlations of polarimetric signals in the line core with the continuum image intensity, scatter plots are shown in Figure 3.4. We find evidence that the Stokes $Q / I$ image in the Sr I line core is anti-correlated with the continuum intensity. To begin with, the correlation is quantified by fitting a linear function to the signals, estimating the slope and its standard error (67\% confidence interval). The values are given at the top of each panel in Figure 3.4. The hint of a correlation in $Q / I$ arises from the comparison of the $Q / I$ correlation in the line core with the correlations in the continuum (shown in the left panels of Figure 3.4) and with $U / I$ and $V / I$. The latter serve as references, as only photon noise is present in these images (see Sect. 3.4.2.3). With -2.76 the slope of $Q / I$ in the line core exceeds more than $18 \times$ its standard error and is higher than the slope in any other polarization state and in the continuum. Higher noise in the line core due to fewer photons than in the continuum is represented by the larger error of the slopes. The weights for the linear fit are linked to the photon noise and assumed to be constant for each data point over the field of view, since the intensity contrast is low.

To show the robustness of the slope against selection bias, we created random subsets with a quarter of the original sample size. The error in the slopes are then $2 \times$ larger than in the full data set. The slopes of the full data set are consistent with the slopes of the subsets within the error margins. We calculated the correlation coefficient for the $Q / I$ case to be $r=-0.17$. The correlation coefficients for the reference images are at least one order of magnitude smaller, see Table 3.1 for a summary. A second statistical test was carried out by calculating the probability for observing a correlation coefficient as large or larger than $|r|$ under the (null) hypothesis that the parent population is uncorrelated. We follow the method in Bevington \& Robinson (2003) where this two-sided probability $p$ for $N$ 
Table 3.1: Pearson's correlation coefficients $r$ and the probability $p$ to observe a value as large or larger than $|r|$ for an uncorrelated population with $N=6105$.

\begin{tabular}{l|c|c|c|c}
\hline \hline Stokes & \multicolumn{2}{|c|}{ continuum } & \multicolumn{2}{c}{ line core } \\
\hline & $r$ & $p$ & $r$ & $p$ \\
\hline$Q / I$ & -0.07 & $<10^{-6}$ & -0.17 & $<10^{-6}$ \\
$U / I$ & 0.01 & 0.43 & 0.02 & 0.12 \\
$V / I$ & 0.02 & 0.12 & 0.00 & 1.0 \\
\hline
\end{tabular}

observations is given by

$$
p(r, N)=2 \int_{|r|}^{1} P(x, N) d x .
$$

We approximated the normalized probability density function $P(r, N)$ for a correlation coefficient $r$ for large $N$ (i.e., $N>300$ ) with

$$
P(r, N)=\frac{\left(1-r^{2}\right)^{(N-4) / 2}}{\int_{-1}^{1}\left(1-r^{\prime 2}\right)^{(N-4) / 2} d r^{\prime}} .
$$

The calculated $p$-values for all polarimetric images are given in Table 3.1. The null hypothesis is rejected if the $p$-value is smaller than 0.001 , which corresponds to a confidence level of $99.9 \%$. We reject the null hypothesis of no correlation for Stokes $Q / I$ in the $\mathrm{Sr}$ I line core, as the two-sided $p$-value is negligible. A small correlation is present for the $Q / I$ continuum image, potentially due to insufficient distance to the Sr I line core. However, the condition $p>0.001$ is fulfilled for the reference images. This situation is maintained when the above mentioned random subsets are analyzed. We are therefore confident that the anti-correlation with $r=-0.17$ is statistically significant.

\subsubsection{Doppler-shift induced horizontal signal fluctuations}

To eliminate signal fluctuations resulting from Doppler shifts, we fitted a Gaussian to the Stokes $I$ spectrum in each spatial pixel. From these fits we calculated a Doppler map, which revealed a mean line core shift of $30 \mathrm{~m} \AA$, while the standard deviation is smaller than our spectral resolution. Therefore the line core signals are largely restricted to a single image, labeled with $30 \mathrm{~m} \AA$ in Figure 3.1. Thus we expect to see scattering polarization signals mainly in the image taken at this wavelength. In the continuum intensity image the dark intergranules and bright granules are distinguishable. Although we refer in this paper to dark and bright features in the continuum intensity as intergranules and granules, we emphasize that these terms are ambiguous due to projection effects off the solar disk center.

The contribution of Doppler-shift-induced fluctuations is on the order of $0.03 \%$ or less. We estimated this upper limit with a simple calculation using the Doppler map mentioned above. The maximum calculated Doppler shift is $23 \mathrm{~m} \AA$ from the mean line core shifts. The standard deviation is $16 \mathrm{~m} \AA$. We initially assumed the same Gaussian shaped Stokes $Q / I$ spectral signal in each spatial pixel located at the mean line core position 


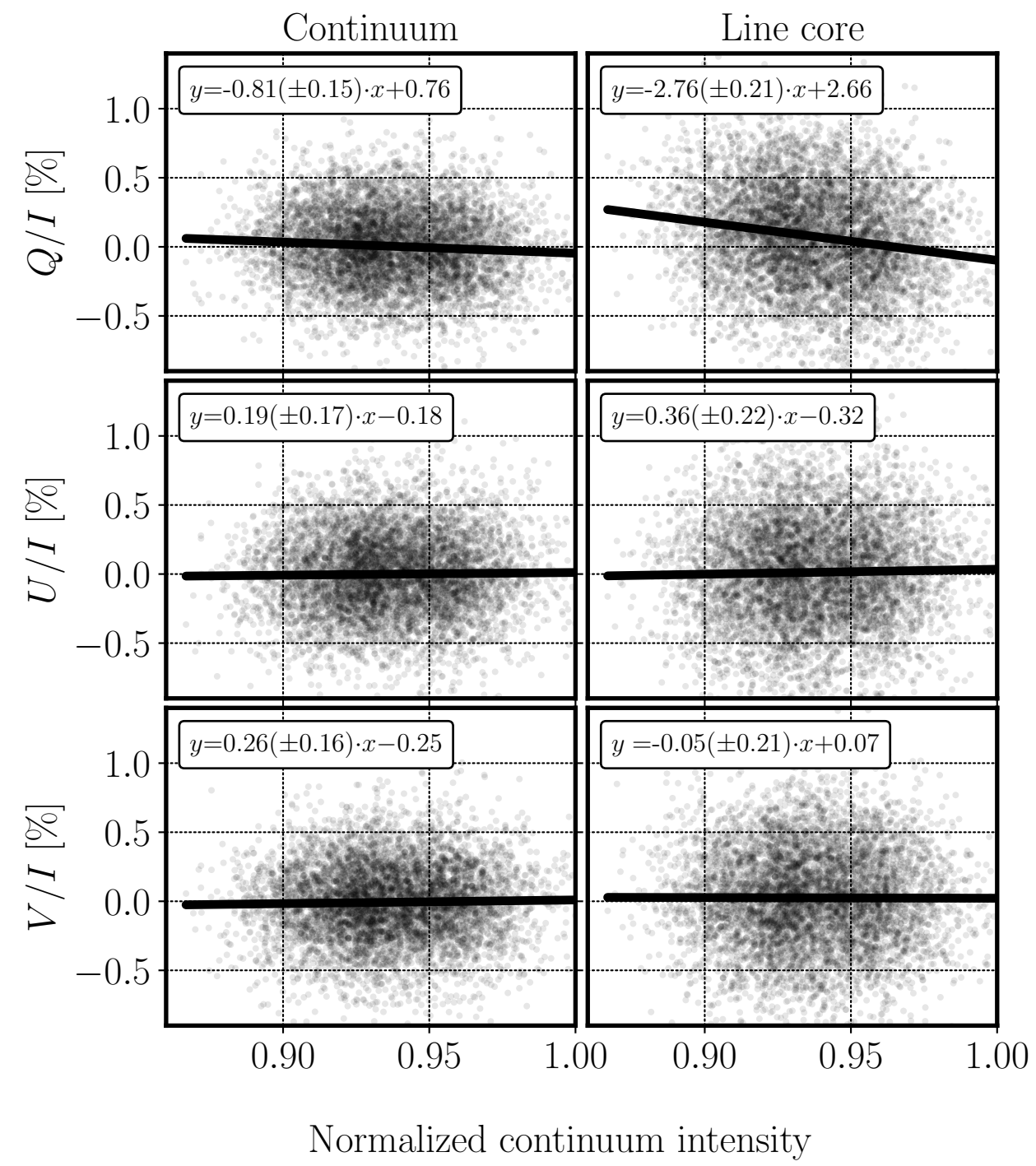

Figure 3.4: Polarization signals of Stokes $Q / I, U / I$ and $V / I$ in the Sr I $4607.3 \AA$ line versus normalized Stokes $I$ continuum intensity. In the left panels the polarization found in the continuum is shown, while in the right panels the polarization signals from the line core are reported. Inserts display the function describing the linear fit, with $x$ as the normalized continuum intensity and $y$ the polarization percentage.

of the spatially resolved data set. Further, we assumed that the $Q / I$ amplitude in each spatial pixel equals $0.19 \%$, this is, the average scattering $Q / I$ signal from the center-to-limb measurement at $\mu=0.6$. A shift of this initial Gaussian by the maximum Doppler shift leads to a drop in the a signal of about $0.03 \%$. Additionally, the correlation between Doppler velocities and continuum intensity is an order of magnitude smaller than between Stokes $Q / I$ and continuum intensity, which also indicates that the anti-correlation between $Q / I$ and continuum intensity is not an artifact. 


\subsubsection{Cross-talk from Stokes I}

We now describe a further test to show that although our Stokes signals are photon noise limited, we still have a clear distinction between Stokes $Q / I$ signals in granules and intergranules. This is an important test to rule out cross-talk from Stokes $I$ to Stokes $Q / I$ as a source of correlation between them. As no polarization is expected in the continuum, we fitted a Gaussian to the probability density function (see left panels of Figure 3.5) to Stokes $Q / I, U / I$ and $V / I$ at the continuum wavelength. To estimate the photon noise expected in the Sr I line core, where less photons are available, the standard deviation of the fitted Gaussian from the continuum was re-scaled.

The re-scaling procedure was done by calculating the parameters (i.e., the amplitude and standard deviation) of the line core Gaussian with two constraints: the first is the ratio of the line core intensity level to continuum intensity level of 0.5 at $\mu=0.6$ (see right panel of Figure 3.5) and the second is that the area below the Gaussian must be unity to get a probability density function.

Based on the continuum intensity image, we classified pixels to granule pixels and intergranule pixels. In order to emphasize the difference between them, we only analyzed a subset of all pixels. Thus granule pixels correspond to the 1000 brightest pixels and intergranule pixels correspond to the 1000 darkest pixels. Stokes signals corresponding to granules and intergranular lanes are plotted in the right panels of Figure 3.5. The signals in $U / I$ and $V / I$ are noise dominated and the Gaussians for the intergranule and granule pixels are indistinguishable. But for the $Q / I$ signals in the interganule pixels, we needed to shift the center of the Gaussian in the right panel of Figure 3.5 from $0 \%$ to $0.14 \%$.

\subsubsection{Influence of spatial resolution}

To test if spatial resolution influences the sign of the line core $Q / I$ slope, we spatially smeared our data by convolving the line core intensity and polarization images with Gaussians with standard deviations between 0.5 pixel and 8 pixels and redid the correlation analysis. We find that the correlation decreases rapidly with the amount of smearing, but does not change its sign. The value of the slope of $Q / I$ versus continuum intensity in the line core when a Gaussian of a rms width of 8 pixels was applied (sampling of 0.64 " per smeared pixel) is reduced to 0.76 . This is close to the slope of $Q / I$ in the continuum at the original spatial resolution. Hence the difference in sign between our results and those of Malherbe et al. (2007) cannot be due to differences in spatial resolution.

\subsubsection{Estimation of polarimetric structure size}

We estimated from our line core Stokes $Q / I$ image that the approximate diameter of structures with scattering polarization signals greater than the noise level is in the range of $0.5^{\prime \prime}$ to $1^{\prime \prime}$. The estimation is based on a two-step analysis of the mean polarization signal, its standard deviation and the expected noise level in size-varying subregions in the image. We describe the steps in more detail in the next paragraph.

In the first step, we defined a subregion in the image as a circular area, having a radius $R$ and containing $N$ pixels, centered on a given pixel in the image. As all pixels were used once as a central pixel, we had in total 6105 subregions per image, independently of the subregion size $N$. A pixel belonged to a given subregion if its Euclidean distance from the central pixel was smaller than $R$. If a central pixel was closer to the image boundary than $R$, 


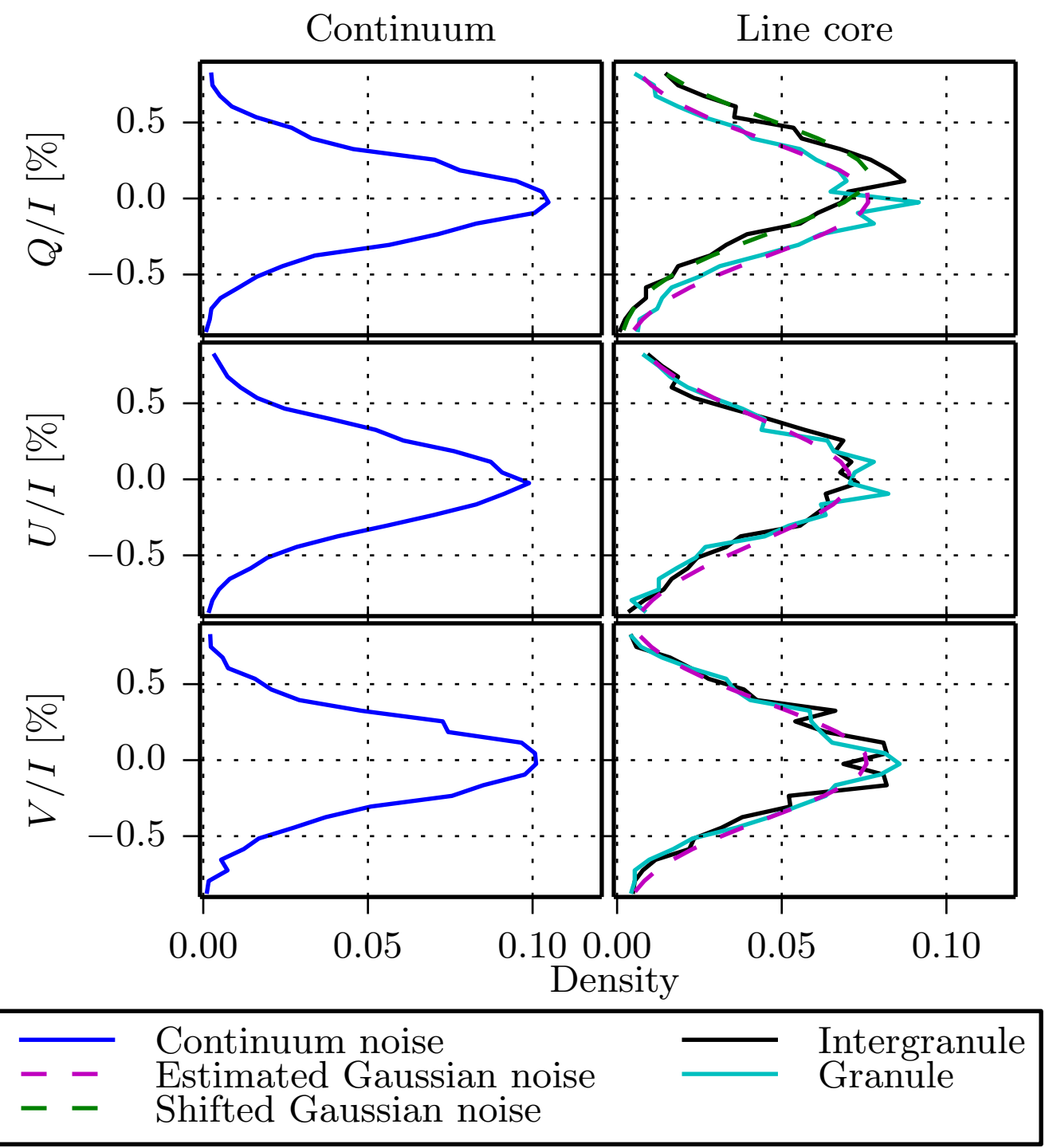

Figure 3.5: Left panels: probability density function of $Q / I, U / I$ and $V / I$ in the continuum. Right panels: noise distribution for the line core of $\mathrm{Sr}$ I inferred from that observed at the continuum wavelength in the various Stokes parameters (magenta dashed lines). The continuum image is classified into granules and intergranules, for which we plotted the Stokes signals separately. A shifted noise distribution (green dashed line) is shown to match the Sr I line core Stokes $Q / I$ signals in the intergranules.

the image was periodically continued. We then varied $R(\propto \sqrt{N})$ and computed the mean polarization signal for each subregion. For each Stokes image and given $N$ we determined the distribution of the number of subregions as a function of average polarization signal using a histogram with bin size of $0.02 \%$. In Figure 3.6 these histograms are depicted as vertical bars with a gray color scale. Black corresponds to 600 subregions and white corresponds to 0 subregions displaying a particular Stokes signal. A Gaussian was fitted to each histogram with the mean $\mu$ and the standard deviation $\sigma$ as free parameters. We 
plot $\mu \pm \sigma(N)$ to each histogram. Additionally, we calculated the noise level based on the single pixel noise $\sigma_{n}(N=1)$ present in each Stokes image (see Figure 3.5). The noise level was reduced according to the number of averaged pixels assuming a shot noise process. According to this assumption, the noise $\sigma_{n}(N)=\sigma_{n}(1) / \sqrt{N}$ dropped with the square root of the number of pixels $N$ in the subregion. The noise level $\mu \pm \sigma_{n}(N)$ is indicated in Figure 3.6 as well.

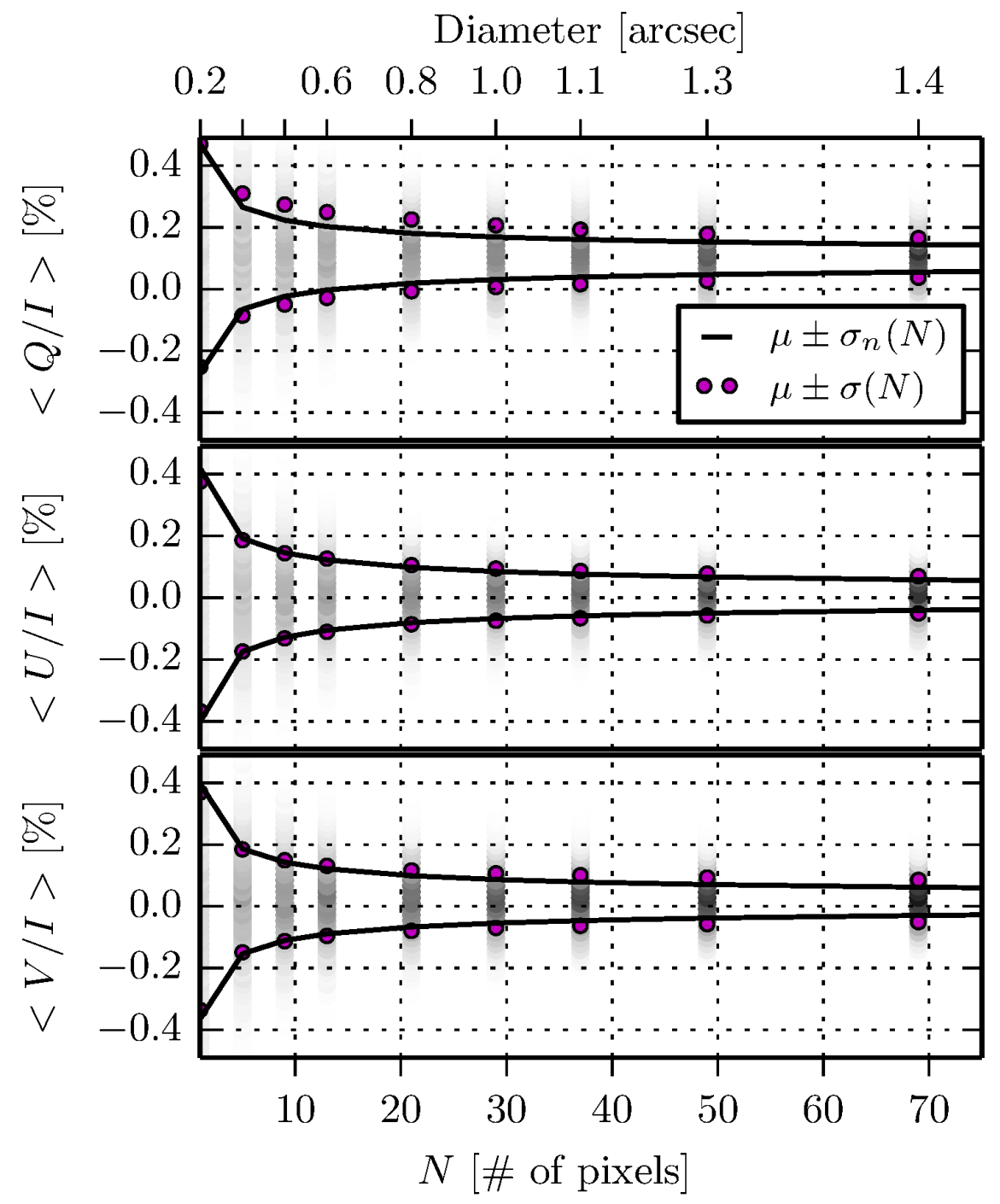

Figure 3.6: Histograms of the average polarization in all subregions with $N$ pixels. The histograms are represented by gray vertical bars, where black and white corresponds to 600 subregions and 0 subregions, respectively. Black lines: expected noise levels for $Q / I, U / I$ and $V / I$ when averaging over $N$ pixels; magenta dots: standard deviation of the histogram for each subregion; see text for details.

For $N=1$ the histograms are comparable to the right panel of Figure 3.5 and are in agreement with the noise level, as expected. A minor offset in the mean of $0.01 \%$ in the $U / I$ and $0.02 \%$ in the $V / I$ image may be due to residual cross-talk from instrument polarization as explained in the earlier data reduction Sect. 3.3.2.2. 
In a second step we considered the absolute differences of the standard deviations of the histograms from the expected noise level. In Figure 3.7 we plot the absolute difference $\Delta$ between the standard deviation $\sigma(N)$ and the expected noise $\sigma_{n}(N)$. The difference for $Q / I$ follows a decreasing trend for subregions with a diameter greater than $0.8^{\prime \prime}$. This means that for subregion areas greater than $0.8^{\prime \prime 2}$ only noise is added. The opposite occurs for $\Delta_{U / I}$ and $\Delta_{V / I}$. The differences increase for subregions with up to $N=40$ pixels. This increase is likely due to small Zeeman signatures present in the data. The non-zero $\Delta$ for greater $N$ hints toward Zeeman signatures at larger scales combined with residual cross-talk errors.

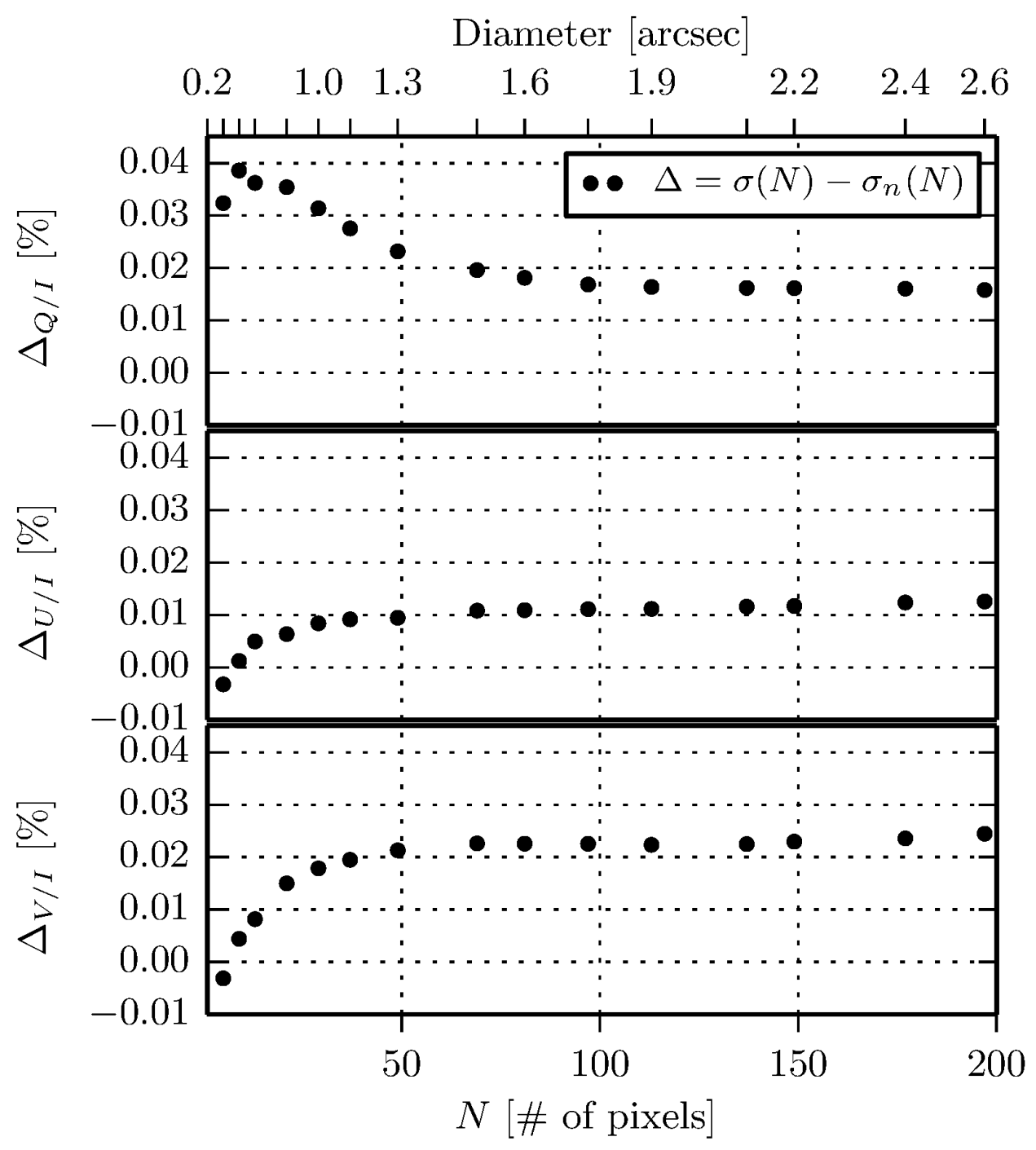

Figure 3.7: Absolute differences between the standard deviation $\sigma(N)$ obtained from the histograms and the expected noise $\sigma_{n}(N)$ for all three polarization Stokes parameters as a function of number of pixels per subregion $N$ (lower axis) and the subregion diameter (upper axis). 


\subsection{Discussion and conclusions}

We performed scattering polarization measurements in the Sr I $4607.3 \AA$ line with the Fast Solar Polarimeter mounted on TESOS at the VTT on Tenerife. To our knowledge, this is the first time a measurement of the scattering polarization in the Sr I line was done with a filtergraph instrument. In a first step, we have tested the reliability of the FSP by comparing the spatially averaged center-to-limb variation of the polarization signal in $\mathrm{Sr}$ I with previous results obtained with other instruments. In addition we verified that the center-to-limb variation of Stokes $Q / I$ in the neighboring scattering-insensitive Fe I $4607.6 \AA$ line is indeed consistent with zero signal. In a second step we analyzed a quiet Sun dataset in the Sr I line recorded at $\mu=0.6$ with a spatial resolution sufficient to clearly separate granules and intergranules in the Stokes $I$ continuum image. After temporal and spatial binning to a nominal resolution of $0.16^{\prime \prime}$ pixel $^{-1}$ and $2.5 \mathrm{~s}$ integration time per wavelength and Stokes parameter, the noise per pixel in the linear polarization is $0.3 \%$. We find a negative correlation of $r=-0.17$ between Stokes $Q / I$ in the line core and the Stokes $I$ continuum intensity. We rule out Doppler-shift induced fluctuations as the source of this correlation. We stress that our measurement is photon noise limited and seeing-induced cross-talk can be ruled out. Despite the small value of the correlation coefficient, our analysis shows statistically robust evidence of an anti-correlation of Stokes $Q / I$ with the continuum intensity in the Sr I line core.

The negative correlation found here seemingly contradicts the positive correlation reported by Malherbe et al. (2007) and Bianda et al. (2018), both obtained by using a spectrograph and at $\mu=0.3$, hence, closer to the solar limb. The correlation coefficient by Bianda et al. (2018) is $r=0.19$, which is close to what has been found in this study, but with opposite sign. The spatial resolution achieved by Malherbe et al. (2007) cannot be better than the used slit width of $0.6^{\prime \prime}$. We speculate that the difference in sign of the correlation found by these authors compared to that observed here could be due to the lower $\mu$ value at which the measurements were made. The contrast and shape of granulation and the sampled atmospheric height range changes considerably with $\mu$. This could lead to different signs of the correlation between Stokes $Q / I$ and continuum intensity. Results by del Pino Alemán et al. (2018) suggest that the positive sign is an artifact due to the reduced statistical significance arising from using a spectrograph.

In order to uncover the cause of different dependences of Stokes $Q / I$ in the line core of Sr I on continuum brightness at different $\mu$ values, both, lower noise, high resolution full Stokes measurements and theoretical calculations of the spatial structure of $Q / I$ in the Sr I line in realistic MHD simulations (Rempel 2014; Vögler \& Schüssler 2007; del Pino Alemán et al. 2018) at multiple $\mu$ values, are needed. From our measurements we furthermore conclude that in order to spatially resolve polarimetric signals the noise level needs to be significantly lower than $3 \cdot 10^{-3}$.

The closest $\mu$ value to our observations at which $Q / I$ images are presented in the most detailed published theoretical study of Sr I scattering polarization in 3D HD simulations is $\mu=0.5$ (Trujillo Bueno \& Shchukina 2007). This polarization map with infinite spectral and high spatial resolution contains patches of polarization as large as $1 \%$, with most of the signals varying between $0 \%$ and $0.7 \%$, in the case where horizontally fluctuating microturbulent magnetic fields in the Hanle saturation regime of $B=300 \mathrm{G}$ in the downflowing intergranules and $B=15 \mathrm{G}$ in granules at all heights, are considered. In the unmagnetized case, but with a degraded spectral resolution of $25 \mathrm{~m} \AA$ and spatial resolution corresponding 
to a $1 \mathrm{~m}$ telescope, the signals vary between $-0.08 \%$ and $0.9 \%$. Patches of granular size with the above mentioned signal levels should be detectable in our data, but are not directly seen. With a statistical approach we estimated the sizes of the scattering polarization structures to be in the range between about $0.5^{\prime \prime}$ to $1^{\prime \prime}$. The obtained size is on the order of the patch sizes of about 1" shown by Trujillo Bueno \& Shchukina (2007). However, a more definite conclusion requires that the simulations be degraded to the same spatial resolution and scattered light conditions as the observations. Nevertheless, finding larger polarization signals in the intergranules is in qualitative agreement with Trujillo Bueno \& Shchukina (2007) and results from a recently published paper by del Pino Alemán et al. (2018). They state that scattering polarization emerges from local fluctuations of the radiation field by plasma inhomogeneities, leading to axial symmetry breaking, even when observed at $\mu=1$. Polarization is therefore predominantly found at the interface of granules and intergranules, with a tendency for stronger polarization in intergranular lanes. Scatter plots provided by del Pino Alemán et al. (2018) show a negative correlation, independent of the $\mu$ value under consideration.

To conclude, in this work we have shown that instruments like FSP are now starting to reach the required combination of polarimetric sensitivity and spatio-temporal resolution to provide first observational feedback to theoretical studies of scattering polarization on small spatial scales. We have presented full Stokes filtergraph observations at $\mu=0.6$ in the Sr I $4607.3 \AA$ line and analyzed the images statistically, as the signal-to-noise ratio was not sufficient to directly detect local fluctuations in the solar scattering polarization signals at spatial scales significantly below 1". From this analysis we found an anticorrelation between the line core Stokes $Q / I$ signals and the continuum intensity. We compared our findings with published observations, which were carried out at $\mu=0.3$ and showed a positive correlation. We additionally compared our result with 3D HD and MHD simulations, where the latter showed a negative correlation independent of the $\mu$ value.

\section{Acknowledgements}

The authors wish to acknowledge fruitful discussions with Rafael Manso Sainz and Ivan Milic. The Fast Solar Polarimeter project is funded by the Max Planck Society (MPG) and by the European Commission, grant No. 312495 (SOLARNET). We also would like to thank the staff of the Kiepenheuer Institut für Sonnenphysik in Freiburg for supporting our FSP observing campaigns at the VTT. We thank all the technical contributors not listed as co-authors for their invaluable input to the project. This project has received funding from the European Research Council (ERC) under the European Union's Horizon 2020 research and innovation program (grant agreement No. 695075) and has been supported by the BK21 plus program through the National Research Foundation (NRF) funded by the Ministry of Education of Korea. The participation of F. Zeuner was funded by the International Max Planck Research School for Solar System Science. 



\title{
4 Paper II: Solar disk center shows scattering polarization in the Sr I $4607 \AA$ line
}

\author{
published on 23.04.2020 in \\ The Astrophysical Journal Letters, Volume 893, Number 2, L44 ${ }^{1}$
}

Franziska Zeuner, Rafael Manso Sainz, Alex Feller, Michiel van Noort, Sami K. Solanki, Francisco A. Iglesias, Kevin Reardon \& Valentín Martínez Pillet

My contribution: Developing the reduction pipeline for the FSP 2 data, reduction, analysis and assisting the observation of the data, writing of the first manuscript draft, development

of the $J_{2}^{2}$-based averaging.

\subsection{Abstract}

Magnetic fields in turbulent, convective high- $\beta$ plasma naturally develop highly tangled and complex topologies — the solar photosphere being the paradigmatic example. These fields are mostly undetectable by standard diagnostic techniques with finite spatio-temporal resolution due to cancellations of Zeeman polarization signals. Observations of resonance scattering polarization have been considered to overcome these problems. But up to now, observations of scattering polarization lack the necessary combination of high sensitivity and high spatial resolution in order to directly infer the turbulent magnetic structure at the resolution limit of solar telescopes. Here, we report the detection of clear spatial structuring of scattering polarization in a magnetically quiet solar region at disk center in the Sr I $4607 \AA$ spectral line on granular scales, confirming theoretical expectations. We find that the linear polarization presents a strong spatial correlation with the local quadrupole of the radiation field. The result indicates that polarization survives the dynamic and turbulent magnetic environment of the middle photosphere and is thereby usable for spatially resolved Hanle observations. This is an important step toward the long-sought goal of directly observing turbulent solar magnetic fields at the resolution limit and investigating their spatial structure.

1 (C) AAS. Reproduced with permission. https://doi .org/10.3847/2041-8213/ab86b8 


\subsection{Introduction}

The observation of magnetic fields on the solar surface is crucial for the empirical study of fundamental astrophysical processes such as magnetoconvection, turbulent dynamos, magnetic reconnection, or plasma energization. The Zeeman effect, the splitting of spectral lines in the presence of a magnetic field and its associated polarized thermal emission, provides a direct spectroscopic technique to detect and diagnose magnetic fields in the solar atmosphere. However, there are fundamental limits for all Zeeman diagnostic techniques to detect magnetic fields that are highly tangled or turbulent and dynamic, because Zeeman polarization patterns from opposite polarities or crossed fields within the observational resolution element cancel out. This makes magnetic fields undetectable in a regime of particular importance, for example, in connection with turbulent small-scale dynamo action and current dissipation.

The Hanle effect, the magnetic modulation of resonance scattering polarization, does not suffer from such fundamental limitations (Stenflo 1994; Landi Degl'Innocenti \& Landolfi 2004). It creates a polarization pattern that depends on the scattering geometry and the magnetic field, even in the case of zero magnetic flux within the resolution element.

The discovery of the linearly polarized component of the spectrum observed close to the solar limb due to scattering was a breakthrough in solar physics that opened the possibility for diagnosing photospheric turbulent magnetic field configurations even at spatial scales below the resolution element (Stenflo 1982; Faurobert-Scholl 1993; Trujillo Bueno 2003a; Manso Sainz et al. 2004; Trujillo Bueno \& Shchukina 2007; Stenflo \& Keller 1996). The Sr I line at $4607 \AA$, one of the strongest scattering polarized lines in the visible, has been widely studied. Its interpretation revealed an ubiquitous hidden magnetic field, which has been proposed to contribute significantly to the energy balance of the Sun (Trujillo Bueno et al. 2004).

To date, observations of scattering polarization on the photosphere have been done mostly close to the solar limb and lack spatial and temporal resolution. In practice, this means that we can neither directly probe magnetic fields nor track dynamo action at the smallest scales. Previous analyses of Hanle signals relied heavily on numerical modeling of the radiation field and required assumptions on the statistical properties of the magnetic field (its probability density function and spatial distribution), both remain largely unconstrained by the low resolution observations. Also, this makes it difficult to create a coherent picture of the magnetic field in the quiet Sun from the complementary Zeeman and Hanle observations.

There is a growing interest in observing scattering polarization at lower heliocentric distances (Malherbe et al. 2007; Bianda et al. 2011, 2018; Zeuner et al. 2018; Dhara et al. 2019) that resolve the polarization fluctuations resulting from the local symmetry breaking of the radiation field with the aim of actually diagnosing the statistical distribution of magnetic fields at the smallest scales. This has been largely motivated by numerical radiative transfer calculations of scattering line polarization in realistic 3D models of the solar atmosphere, showing that horizontal inhomogeneities may indeed produce measurable linear polarization patterns at granular scales (Trujillo Bueno \& Shchukina 2007; del Pino Alemán et al. 2018). This is of diagnostic value for small-scale dynamos and magnetoconvection (e.g., Vögler \& Schüssler 2007; Rempel 2014). Observing this is a challenge because the polarimetric signals are weak and the high spatio-temporal resolution required 
severely limits the number of available photons in a way that cannot be compensated easily through standard techniques. Unlike intensity, polarization is quantified through signed quantities and simple averaging (either by low spatial resolution or post-processing) over areas characterized by very inhomogeneous radiation fields may lead to cancellations rather than signal enhancement. Therefore, observations have to provide at least subgranular resolution.

This Letter reports the detection of spatially structured scattering polarization signals in the Sr I $4607 \AA$ line in a quiet Sun region at disk center. It relies on a novel analysis technique that enhances weak scattering polarimetric signals using a reconstruction technique of the pattern of the local quadrupole moment of the radiation field inferred from the observed intensity map itself. We show that a reconstruction based on geometric considerations is sufficient for this purpose; therefore, our work is independent of models that include either magnetohydrodynamic or detailed radiative transfer calculations.

\subsection{Observational data}

The observations were carried out with our new high-cadence Fast Solar Polarimeter 2 at the Dunn Solar Telescope (see Appendix 4.7.1 for more details on the instrument and observation), which provides increased polarimetric sensitivity $(<0.1 \%)$ while conserving sufficient spatio-temporal resolution to resolve subgranular scales (see Figure 4.1). We successively observed in three wavelength positions: the Sr I line core, the core of a neighboring Fe I line and a continuum position. The integration time at the former two positions was $210 \mathrm{~s}$, while in the continuum it was $120 \mathrm{~s}$. The two latter positions serve as references. The filter was spectrally broad enough to average substantially over the line profiles (see Appendix 4.7.1 for the spectral filter profile). After data reduction (see Appendix 4.7.2 for the data reduction steps), we have $42(24) \times 5$ s averaged images per Stokes parameter in the line cores (continuum). We may safely assume that we capture an instantaneous solar scene in each $5 \mathrm{~s}$ image, compared to typical solar evolution times of around $30 \mathrm{~s}$ in the quiet Sun at the spatial scales of our observation. The observed region was located close to solar disk center $(\mu=\cos (\theta)=0.98, \theta$ is the heliocentric angle) and corresponded to the quiet Sun internetwork. We checked the quietness of the region in H- $\alpha$ before we carried out the observation. 

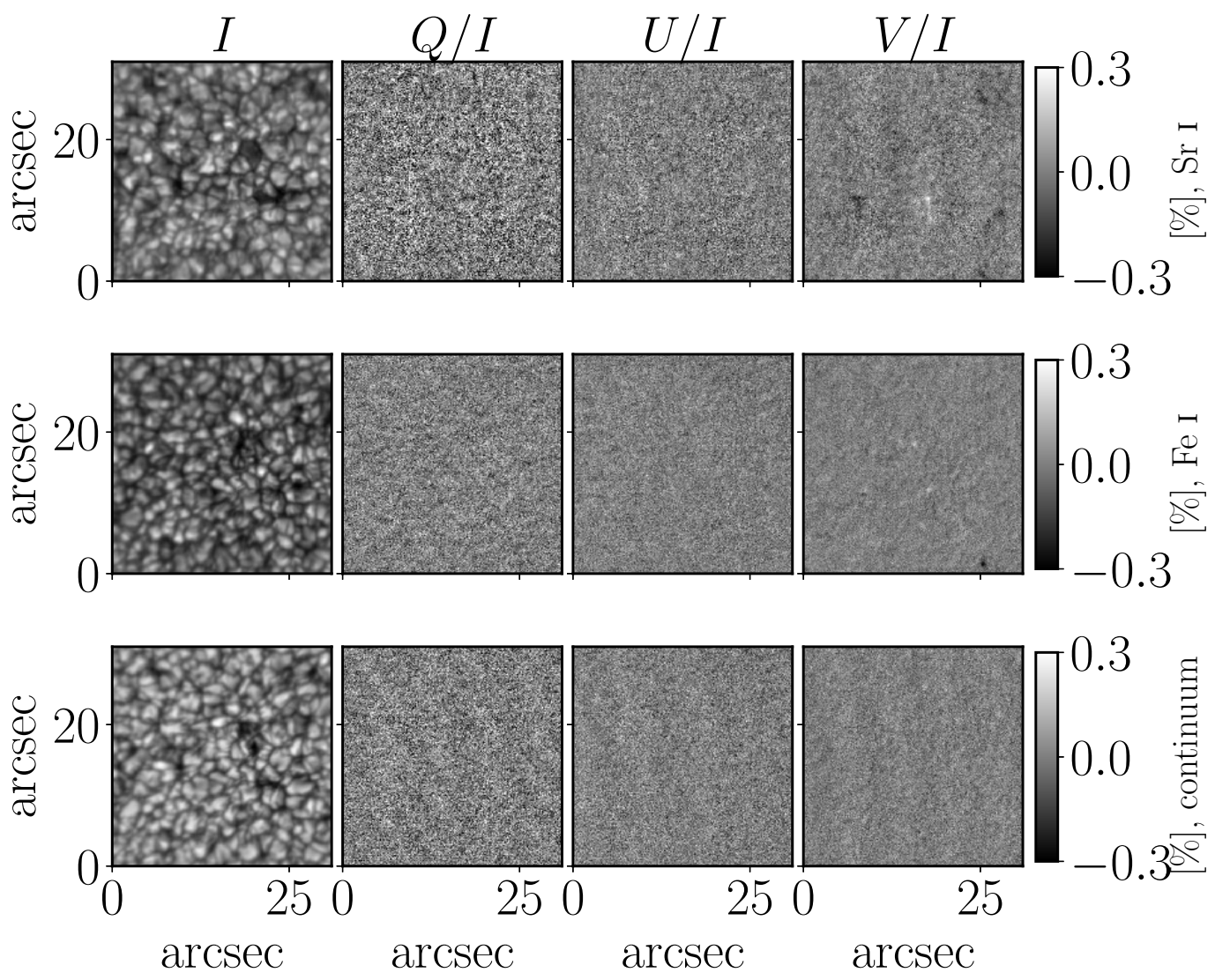

Figure 4.1: Time-averaged data set (over the entire observation period), consisting of the complete Stokes vector (columns) at the three different recorded wavelength positions (rows). The gray scale refers only to the normalized polarization Stokes images that have an RMS noise level on the averaged images of $\sim 0.03 \%$. The root-mean-square (RMS) contrast for the Stokes $I$ images is $\sim 6 \%$. Granulation is clearly distinguishable in the temporally averaged images, showing the high quality of the data and the uninterrupted stable seeing conditions during the full observation. The seeing conditions in all wavelength positions were almost identical, allowing a fair comparison between the different data sets. Note that the $Q / I$ and $U / I$ Sr I core images are practically free of residual artifacts such as low-amplitude fringe patterns, as the data reduction steps are optimized for this wavelength position and are then applied to the data of the other two positions. Localized patches of both $V / I$ polarities in the core of the Sr I and Fe I lines are visible and of solar origin (longitudinal Zeeman signatures).

\subsection{Pixel classification based on the local quadrupole of the radiation}

The scattering geometry for an observation at disk center is presented in Figure 4.2. The radiation scattered in the Sr I line is always linearly polarized, when incoming and scattered beams are perpendicular to each other. Therefore, incoming radiation from east and west is scattered toward the observer with $Q>0$, and radiation from north and south, with $Q<0$ 
— similarly for $U$, but for the direction of the incoming radiation turned by $45^{\circ}$ (panel (b) of Figure 4.2). Net linear polarization is therefore a result of an axially asymmetric illumination, e.g., $Q>0$ if the east-west component of the radiation field dominates. More quantitatively, the combination of the Stokes parameters $Q+\mathrm{i} U$ of the scattered radiation is proportional to the quadrupolar component, $-\sqrt{3} J_{2}^{2}$, of the incoming radiation field (see Figure 4.2; Chandrasekhar 1960; Landi Degl'Innocenti \& Landolfi 2004). We use a simple scattering model to estimate the real and imaginary components of $J_{2}^{2}=\tilde{J}_{2}^{2}+\mathrm{i} \hat{J}_{2}^{2}$ at any point in the field of view and at every time step from the respective observed intensity map. Note that due to the broad pre-filter the observed intensity maps, even at the line core wavelength positions, are dominated by the continuum intensity. The scattering model is simplified by using the following assumption: that a thin scattering layer is at a height $h$ above an atmosphere that provides incoming radiation given exactly by the observed intensity map. Thereby, we neglect the three-dimensional structure of the Sun's surface. In the following we will describe the procedure in more detail. The complex quadrupolar component of the radiation field characterizes the incoming radiation on a scatterer (Landi Degl'Innocenti \& Landolfi 2004):

$$
J_{2}^{2}=\frac{\sqrt{3}}{4} \oint \frac{\mathrm{d} \Omega}{4 \pi} \sin ^{2} \theta \mathrm{e}^{-2 \mathrm{i} \chi} I(\theta, \chi) .
$$

The integral is over the unit sphere $\mathrm{d} \Omega=\mathrm{d} \mu \mathrm{d} \chi=\cos \theta \mathrm{d} \theta \mathrm{d} \chi$ with the polar and azimuthal components $\theta$ and $\chi$, respectively (for $\theta=0$, the incoming radiation is vertical, see Figure 4.2 for the geometry). Only the intensity $I$ is relevant here, additional contributions from polarization are negligible. The component $J_{2}^{2}$ may be estimated by considering a thin, uniform scattering layer model, at a height $h$ above an atmosphere that radiates isotropically ${ }^{2}$ and radiates exactly like the observed intensity map. The intensity $I(\theta, \chi)$ incoming on the scatterer at $(0,0, h)$ (in our model $h$ is in units of a pixel) from a ray along the direction $\theta$ and $\chi$, is then exactly given by the observed intensity at the point $-h \tan \theta(\cos \chi, \sin \chi, 0)$ where the ray path intersects the underlying atmosphere. This is the only point where the parameter $h$ enters the equation 4.1. Numerical integration over the outgoing hemisphere provides $J_{2}^{2}$ at any given point in the field of view (FoV). At the edge of the FoV we assume periodic boundary conditions.

The value of the single parameter in the model is set to $h=3$ pixels, because this is where the $J_{2}^{2}$ maps show the greatest contrasts (see Appendix 4.7.3). Regions with similar illumination (similar $J_{2}^{2}$ ) are expected to produce similar values of $Q$ and $U$, up to some factor times the random noise.

In order to increase the polarization signal while decreasing the noise level, we follow a two-step process. First, we calculate $\tilde{J}_{2}^{2}$ and $\hat{J}_{2}^{2}$ based on the $5 \mathrm{~s}$ intensity images for each pixel in the FoV as explained in Figure 4.2 and above. As we are not interested in the absolute values of $J_{2}^{2}$, we normalized $J_{2}^{2}$ according to the maximum value occurring during the complete time series. Therefore, the $J_{2}^{2}$ values lie between \pm 1 .

Second, we temporally and spatially average the observed polarization only among pixels with similar values of $J_{2}^{2}$ (i.e., within 0.05 in the [-1,1] range). This process circumvents spatial smearing of the linear polarization signals during solar evolution while decreasing the noise level significantly.

\footnotetext{
${ }^{2}$ Consequentially of this assumption, the center-to-limb variation of the underlying radiation field is neglected.
} 


\section{a}
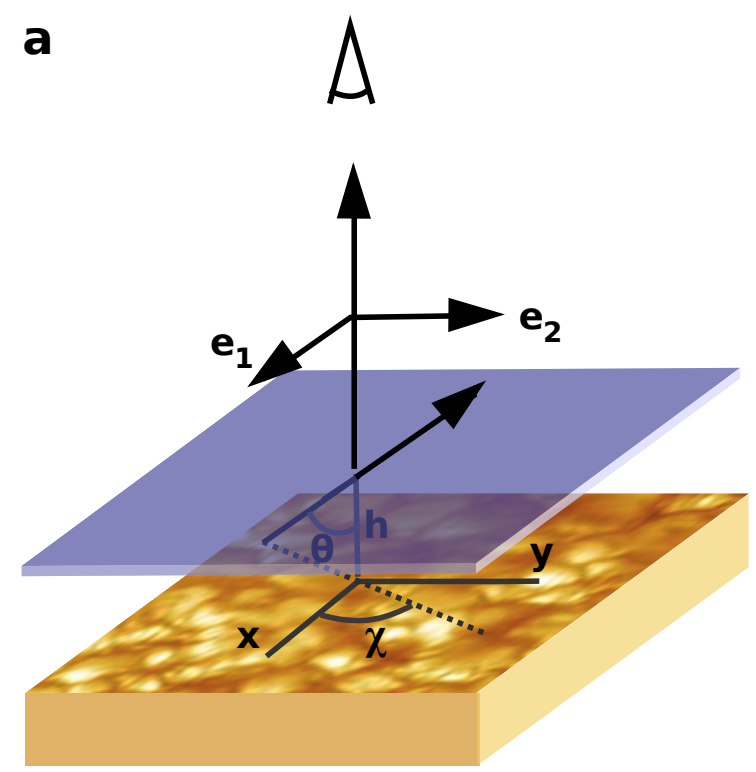

b
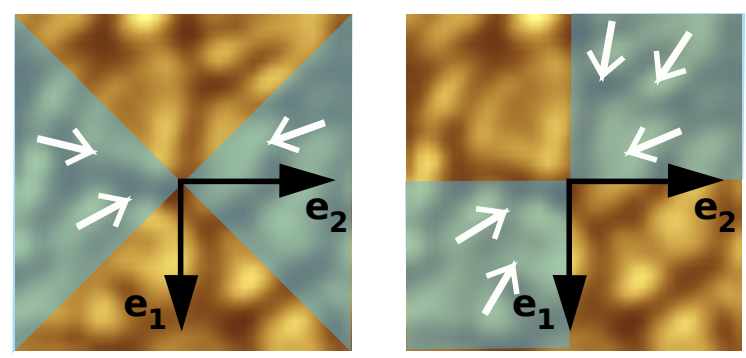

Figure 4.2: Scattering polarization at the center of the solar disk arises from the axial symmetry breaking of the radiation field produced by inhomogeneities in the atmosphere. a) For a given point in the field of view, we use the observed intensity map dominated by the continuum and choose a reference system with the $x$-axis along the positive- $Q$ direction $\left(\boldsymbol{e}_{1}\right)$ and the $z$-axis along the line of sight. The $Q$ direction is given by the polarimeter used for the observation. The radiation, which hits a scatterer in an elevated thin and uniform layer (schematized by the blue surface) at a height of $h$ above the observed intensity map, is represented by the complex quadrupolar component of the radiation field $\left(J_{2}^{2}\right)$. To estimate $J_{2}^{2}$ with equation 4.1 , we use the observed intensity map as a proxy of the incoming radiation field below the scattering layer. Each ray of this radiation field hitting a scatterer can be characterized by the height $h$ and the azimuthal and polar angles $\chi$ and $\theta$, respectively. See the main text for details. b) As seen by the observer along the scattered direction, radiation incoming from the areas marked with white arrows (e.g., east-west for Stokes $Q$ ) contributes to positive $Q\left(\operatorname{Re}\left(J_{2}^{2}\right) \equiv \tilde{J}_{2}^{2}<0\right.$; left panel), or positive $U\left(\operatorname{Im}\left(J_{2}^{2}\right) \equiv \hat{J}_{2}^{2}<0\right.$; right panel) signals.

\subsection{Results}

According to the above picture, a scattering dominated $Q$ signal should anticorrelate strongly with $\tilde{J}_{2}^{2}$ (the larger the illumination from east-west with respect to north-south, the 
smaller (more negative) $\tilde{J}_{2}^{2}$ and the larger the value of $Q$ ). Following a similar argument, $U$ should anticorrelate with $\hat{J}_{2}^{2}$. In the presence of a magnetic field, these correlations change in general. A magnetic field along (or away from) the line of sight in the Hanle regime, for example, rotates theses patterns so that for increasingly stronger fields, $Q$ and $U$ tend to anticorrelate with $\hat{J}_{2}^{2}$ and $\tilde{J}_{2}^{2}$, respectively. However, for an isotropic distribution of magnetic fields, we recover the behavior of the pure scattering case. The difference between the isotropic field and the pure scattering case lies in decreased amplitudes of $Q$ and $U$ compared to the zero-field case. This is exactly what we find.

Figure 4.3 shows the $Q / I$ and $U / I$ in the $\hat{J}_{2}^{2}-\tilde{J}_{2}^{2}$ plane, when temporally and spatially averaged as explained in the last section. The core of the Sr I $4607 \AA$ line clearly shows the anticorrelation between $Q / I$ and $\tilde{J}_{2}^{2}$, as well as between $U / I$ and $\hat{J}_{2}^{2}$. Such a pattern is barely noticeable in the continuum, the core of the Fe I line, or net circular polarization $(V / I)$. We tested that the pattern in the Sr I line is not an artifact of the analysis technique by repeating exactly the same process but randomly scrambling the positions of the pixels in the observed polarization images. In this case, the coherence between polarization and radiation field structure disappears (rightmost panels in Figure 4.3).

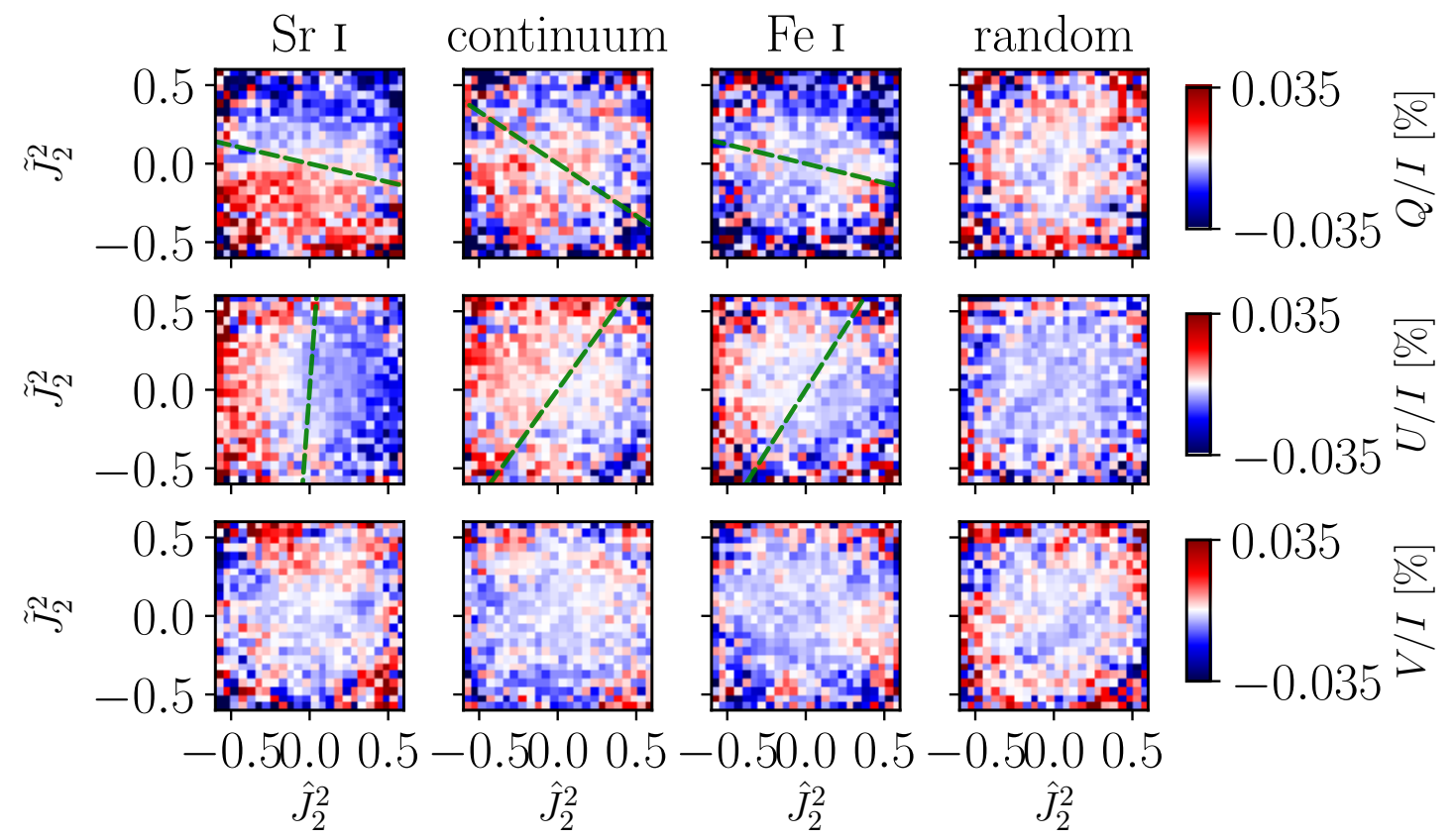

Figure 4.3: Average (as explained in the section 4.4) observed polarization as a function of the normalized radiation's quadrupolar component $J_{2}^{2}$ (see the main text for explanation) for all three observed wavelength positions and for randomized Sr i polarization images (from left to right). Here, we display only the polarization in the limited range of $-0.55 \leq$ $J_{2}^{2} \leq 0.55$, where most pixels lie. Green dashed lines show the nodal lines, which we get from a weighted two-dimensional linear regression fit (see the main text for details). For the regression, $Q / I$ and $U / I$ were fit independently and their nodal lines (green dashed lines) appear appear roughly orthogonal and barely rotated in the $\tilde{J}_{2}^{2}-\hat{J}_{2}^{2}$ plane within observational uncertainties.

The residual $U / I$ feature in the reference wavelength positions (Fe I core and continuum) 
is most likely an artifact due to the broad pre-filter in the observation setup, which in combination with the expanded tails of the spectral profile of the Fabry-Pérot system spectrally contaminates the reference wavelengths with $\mathrm{Sr}$ I signals. Stokes $Q / I$ in the reference wavelength position is probably missing an obvious residual feature like in Stokes $U / I$, as the noise level is higher in this Stokes parameter compared to $U / I$ (see Appendix 4.7.2 for an explanation). We exclude the possibility that the features arise as residual Stokes I cross-talk or the transverse Zeeman effect, since the reference wavelength positions should otherwise also show them as strongly as in the $\mathrm{Sr}$. Hence, we are confident that the linear polarization signals obtained after averaging are actual scattering polarization signals in the core of the Sr I line at the center of the solar disk. In addition, Figure 4.3 provides evidence that the polarization signal is structured with respect to the underlying radiation field.

Here we do not try a detailed spectropolarimetric modeling of the observations to infer the properties on the observed region; rather, we consider the qualitative, more fundamental implications of this detection: that the atomic alignment induced in the excited level, ${ }^{1} P^{\circ}$, of $\mathrm{Sr}$ I by anisotropic scattering is not destroyed by depolarizing effects (either from collisions or magnetic fields), even in the deep, dense regions of the photosphere probed at disk center.

Vertical magnetic fields are very efficient at depolarizing and rotating the plane of scattering polarization from resolved horizontal inhomogeneities observed at disk center (Manso Sainz \& Trujillo Bueno 2011). To find the rotation angle of the scattering polarization plane with respect to the radiation field, we fit the plane $L=\alpha \cdot \hat{J}_{2}^{2}+\beta \cdot \tilde{J}_{2}^{2}$ to the polarization values in each panel in Figure 4.3 independently, without considering an offset, as the mean polarization was subtracted in the data reduction. The nodal lines, i.e., where $L=0$, indicate where the observed linear polarization is close to zero. In the Sr I line core, the $L=0$ lines are nearly horizontal, and almost perpendicular to each other for Stokes $Q / I$ and $U / I$, respectively. We do not find evidence that the small rotation in Figure 4.3 in the Sr I data, indicated by a mismatch of nodal lines $Q / I=0$ and $U / I=0$ with $\hat{J}_{2}^{2}=0$ and $\tilde{J}_{2}^{2}=0$, respectively, has any other source than noise. We tested this by adding a few realizations of random noise (with the same RMS as the observation itself) to the observation and repeat the fitting process for each realization. We find an angular uncertainty of about $5^{\circ}$.

Our findings (scattering polarization but no significant Hanle rotation) advocate two possible scenarios. If the photosphere is dominated by vertical magnetic fields, most of them must be well below the saturation regime - otherwise, the polarimetric signal would vanish completely, while from the lack of significant rotation of the polarization plane we conclude that such a vertical distribution of fields has mixed polarities. The RMS of the magnetic field strength $\Delta B_{\text {rms }}$ over the observed field of view is such that $2\left(3 \Delta B_{\text {rms }} / 22.8 \mathrm{G}\right) \lesssim 10$ in the collisionless limit (here, the critical field $22.8 \mathrm{G}$ appears from expressing the Larmor frequency in units of the Einstein coefficient for spontaneous emission $A_{u \ell}=2 \times 10 \times 10^{8} \mathrm{~s}^{-1}$, a factor of two is related to the quadrupolar component of the radiation field and the majority of magnetic field strength values, assuming a Gaussian distribution, lies between $\pm 3 \Delta B_{\mathrm{rms}}$ ). Collisions contribute additionally to relax atomic polarization and the critical field increases by a factor of $(1+\epsilon+\delta)$, where $\epsilon$ and $\delta$ are the rates of inelastic and elastic depolarizing collisions, respectively, normalized to $A_{u \ell}$ (Landi Degl'Innocenti \& Landolfi 2004). 
At typical formation heights of the Sr I line (at around $170 \mathrm{~km}$ above optical depth unity, see del Pino Alemán et al. 2018), $\epsilon$ is negligible (e.g., van Regemorter 1962) while depolarizing collisions are significant (e.g., del Pino Alemán et al. 2018): $\delta \approx 4$ (FaurobertScholl et al. 1995) $-\delta \approx 2$ (Manso Sainz et al. 2014; del Pino Alemán et al. 2018). Note that with increasing $\delta$, the scattering polarization generated is also reduced and if $\delta \gg 1$, it would be completely wiped out. Therefore, our observations are compatible with a photosphere filled with (nearly) vertical magnetic fields with $\Delta B_{\mathrm{rms}} \lesssim(38 \mathrm{G})(1+\delta) \approx 114$ $190 \mathrm{G}$. Alternatively, the magnetic field could adopt a more general distribution, inclined to the vertical and having uniformly random azimuths. Our observational setup does not allow us at present to constrain horizontal magnetic fields. The vertical component depolarizes the scattering signals but a net polarization still emerges from regions dominated by the horizontal magnetic fields.

For example, an isotropic, Maxwellian distribution of magnetic field strengths (average field strength $\bar{B}$ ), results in a decrease of observed scattering polarization signal (with respect to the maximal, zero-field value). The observed scattering polarization amplitude decreases with increasing $\bar{B}$ but saturates at $20 \%$ of the original polarization for $\bar{B} \gtrsim 130 \mathrm{G}$.

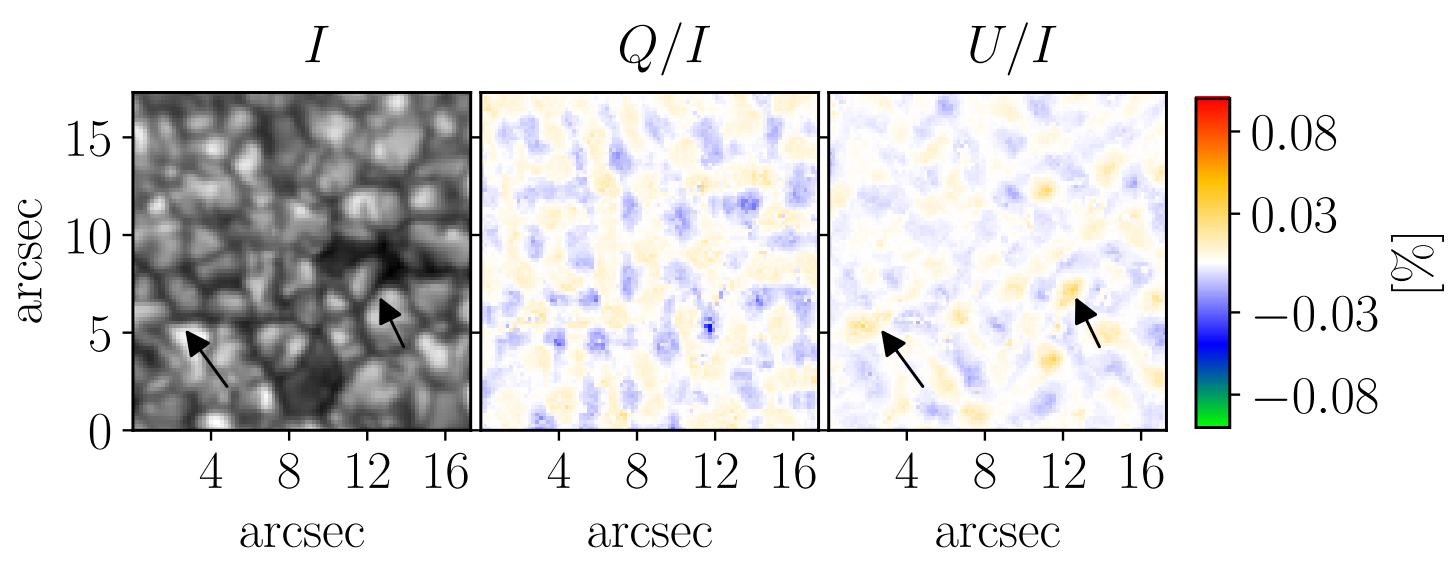

Figure 4.4: Stokes I and "reconstructed" linear polarization maps in the Sr I line core. From left to right: temporally averaged observed intensity image (section of the top left frame of Figure 4.1), reconstructed $Q / I$ and $U / I$ spatial maps from the polarization in Figure 4.3. These maps have been reconstructed by retracing the averaged polarization values from Figure 4.3 to their original positions in an observed $5 \mathrm{~s}$ Stokes $I$ image (i.e., to the places with the corresponding $J_{2}^{2}$ values) and then (again) temporally averaging over the full period of observation. The reconstruction can be considered to be equivalent to a clever way of denoising the observations.

Figure 4.4 shows a reconstruction of the spatial distribution of the polarization signals. The reconstruction has been obtained by retracing the averaged polarization values shown in Figure 4.3 to each spatial pixel according to its local value of the radiation field, i.e., for each $5 \mathrm{~s} J_{2}^{2}$ map. Then we temporally averaged the reconstructed maps to achieve comparability to Figure 4.1. Note that multiple spatially different pixels in the reconstructed map may therefore have the same polarization values due to the prior averaging in Figure 4.3. 
The spatial structure of the polarization signals is subgranular. They are mostly located at the interface between granules and intergranules. Notice that large signals with $U / I \sim 0.05 \%$ are visible and not limited to individual pixels, but extend to a size of about an arcsecond (marked with arrows in the $U / I$ reconstructed image and in the same spatial positions in the Stokes $I$ image to emphasize that the signals are located close to the interface between granules and intergranules).

An upper limit of the average scattering structure size is $0.75^{\prime \prime}$ (which was found by convolving spatially with Gaussians of increasing widths until the resultant polarization amplitude in the $\tilde{J}_{2}^{2}-\hat{J}_{2}^{2}$ plane is in the order of the reference wavelengths). However, larger structures than $0.75^{\prime \prime}$ are occasionally possible, see Figure 4.4 . These structure sizes are consistent with the previous estimate of $0.5^{\prime \prime}-1^{\prime \prime}$ (Zeuner et al. 2018).

The mean absolute linear polarization for the Sr I line core in Figure 4.4 is $0.005 \%$ and $0.003 \%$ in Stokes $Q / I$ and $U / I$, respectively, while the standard deviation is $0.006 \%$ and $0.005 \%$. These values obviously correspond to the finite spectral and spatial resolution of the observations and we expect higher signals with increased spatial and in particular, spectral resolution. del Pino Alemán et al. (2018) report disk center polarization amplitudes of up to $1 \%$ in synthetic Stokes profiles from recent magnetoconvection models of the quiet solar photosphere.

\subsection{Discussion and conclusion}

We have shown here how it is possible to map the scattering polarization component of radiation at solar disk center by employing the information available on the inhomogeneous solar surface. With our observational setup we find absolute polarization amplitudes of typically $\sim 0.004 \%$, corresponding to standard deviations of below $0.01 \%$, while the mean spatial extent is limited to sizes smaller than $0.75^{\prime \prime}$. It is known that the amplitude of the scattering polarization, in particular, is sensitive to the spectral resolution of the observation. This has been studied in detail by del Pino Alemán et al. (2018). In our case, the spectral resolution was about $67 \mathrm{~m} \AA$ FWHM and the polarization amplitude is even below the noise level of the observed polarization images, which gives a signal-to-noise ratio of less than one. This explains the absence of distinct polarization signals in the observed polarization images. The most striking result is that we find the scattering polarization to be anticorrelating with the radiation field's quadrupole tensor element $J_{2}^{2}$ estimated from the observed Stokes $I$ map, which is dominated by the continuum radiation in our data. We also find that the spatial structure of the scattering polarization is subgranular, in agreement with the theoretical predictions by Trujillo Bueno \& Shchukina (2007) and del Pino Alemán et al. (2018). This means that atomic polarization is not destroyed completely even in very dense layers of the quiet solar photosphere, confirming the results from numerical calculations reported by Trujillo Bueno \& Shchukina (2007); del Pino Alemán et al. (2018).

We find that the observed scattering polarization is compatible with two alternate scenarios of the magnetic field's structure in the quiet photosphere. Either the magnetic field in the photosphere is dominantly vertical, with strengths below the saturation regime of $114-190 \mathrm{G}$, depending on the collisional rates and of zero mean flux. Alternatively, the 
magnetic field can be much stronger if it is more horizontal on average. These findings are not constrained by the specific spatial resolution of the observation, as the Hanle effect does not suffer from subresolution cancellations. However, resolving subgranular scales is necessary in order to resolve the polarization emerging from the axial symmetry breaking of the radiation field introduced by the thermal inhomogeneity due to granulation. Investigations based on the Hanle effect (Trujillo Bueno et al. 2004; del Pino Alemán et al. 2018) and Zeeman effect-based analyses (e.g. Lites et al. 2008; Danilovic et al. 2010a, 2016) have returned average field strengths reaching $100 \mathrm{G}$ or more in the quiet Sun. Such significant average field strengths are consistent with our analysis, which are also found in recent magnetoconvection simulations with small-scale dynamo action (Vögler \& Schüssler 2007; Schüssler \& Vögler 2008; Rempel 2014; Khomenko et al. 2017), particularly in the middle photosphere, where the Sr I line is formed. In the future, we expect stronger constraints by combining spatially resolved observations at different limb distances.

The new polarimeter combined with the simple scheme used here to extract the signal from the noise was designed as a proof-of-principle experiment. Better results are expected from an improved observational setup, e.g, by utilizing a narrower filter to minimize the contamination of signals between lines and also the continuum; ideally, spectropolarimetry would avoid such contamination entirely. The novel technique employed here for the first time cleverly trades spatial information in the whole FoV for polarimetric sensitivity. Therefore, there is much to gain from new advanced instrumentation in upcoming facilities, such as the Daniel K. Inouye Solar Telescope currently being tested on Maui, Hawaii, that offer an extended FoV, increased photon flux, and yet high spatial resolution. Alternatively, extended time series, even of restricted FoV's, can now be integrated without loss of polarimetric signal. Once any of this is achieved and we are able to detect the polarimetric signal and to quantify its statistics reliably, it will be possible to diagnose the magnetic field from the Hanle signals solely from the statistics of the data in a totally model independent manner. Spatially resolved scattering polarimetry observation on the solar disk is one step closer to delivering on the promise of Hanle effect diagnostics in the photosphere that are complementary to, but potentially on par with, state-of-the-art Zeeman techniques in spatial and temporal resolution.

\subsection{Appendix to Paper II}

\subsubsection{Observation}

All four Stokes parameters were recorded in a quiet Sun region very close to disk center $(\mu=0.98$ ) on 2017 August 8 between 14:41 and 15:20 UTC. The adaptive optics system was locked on the granulation. The observations were performed with the FSP 2, which is briefly described below. The polarimeter was integrated into a single-etalon collimated setup at the 0.76 m Dunn Solar Telescope (NSF's DST), located on Sacramento Peak in Sunspot, New Mexico.

The Fast Solar Polarimeter (FSP) is a ground-based solar polarimeter designed to provide fast modulation and high frame rates to allow for suppression of seeing-induced cross-talk and image restoration at the same time. Additionally, high polarimetric accuracy 
is achieved. For a detailed description of the prototype of FSP, see Iglesias et al. (2016). In the second generation of FSP, used to obtain the data analyzed here, the pnCCD of the prototype of FSP was replaced by a $4 \mathrm{k} \times 3 \mathrm{k}$ CMOS sensor. The frame rate was set to $200 \mathrm{~Hz}$. To enable a dual-beam configuration on a single sensor, a custom designed ${ }^{3}$ polarizing beam-splitter was attached to the CMOS camera. The camera will be described in more detail below. The combined instrument, consisting of the modulator (Iglesias et al. 2016), the polarizing beam-splitter, and the camera is called FSP 2.

The CMOS camera attached to the beam-splitter is a commercially available camera, based on the CMOSIS CMV-12000 image sensor, which has been customized to include a sensor cold finger combined with a thermo-electric temperature control, water cooling, and a sensor mask to shield border pixels for calibration purposes. The temperature of the sensor is stabilized to $20^{\circ} \mathrm{C}$ to better than $\pm 0.5 \mathrm{~K}$. The pixel area is $5.5 \mu \mathrm{m}^{2}$. The camera has a readout noise of $13 \mathrm{e}^{-} \mathrm{RMS}$. With a nonlinearity term of up to $2 \%$, the low contrast images of the quiet Sun are affected by telescope induced polarization, that adds spurious signals proportional to Stokes $I$ (Keller 1996) to the normalized polarization data. Correction of the imprinted and scaled Stokes $I$ by simple subtraction results in a higher noise level compared to data that are unaffected by telescope polarization. The scaling factor for correcting this Stokes $I$ cross-talk is estimated and explained in more detail in Appendix 4.7.2. The data depth is 10 bit. The camera is directly attached to the beam-splitter, which prevents relative motion between these two components. Furthermore, the separation line of the channels in the dual-beam setup divides the camera sensor into two halves. To achieve the desired frame rate of $200 \mathrm{fps}$ (modulation frequency was $50 \mathrm{~Hz}$ to obtain full Stokes measurements), the total readout area of the sensor was reduced to $2048 \times 2048$ pixel $^{2}$. Consequently, when critically sampled at $4607 \AA$ (the plate scale is $0.062^{\prime \prime}$ pixel $^{-1}$ ), each dual-beam channel exhibits a field of view of $63^{\prime \prime} \times 126^{\prime \prime}$.

For wavelength discrimination, a single Fabry-Pérot etalon was used in a collimated setup with a spectral full-width at half-maximum (FWHM) of $67 \mathrm{~m} \AA$. The free spectral range is $1.75 \AA$. The pre-filter FWHM is $169.7 \mathrm{~m} \AA$. This means, that secondary transmission peaks are insufficiently suppressed and spectral stray light affects the data, decreasing the SNR. The total normalized spectral profile for the Sr I line core position of the FabryPérot is shown in Figure 4.5 (secondary peaks are not visible in the displayed narrow frequency window).

We sampled three wavelength positions around the Sr I line, which included a wavelength position very close to the $\mathrm{Sr}$ I line core, the neighboring $\mathrm{Fe}$ I line core, and a continuum point, displayed in Figure 4.5.

\footnotetext{
3 Max Planck Institute for Solar System Research in collaboration with LightMachinery Inc. The extinction ratio over the entire wavelength range of $400 \mathrm{~nm}-860 \mathrm{~nm}$ is better than 1:40 in both channels.
} 


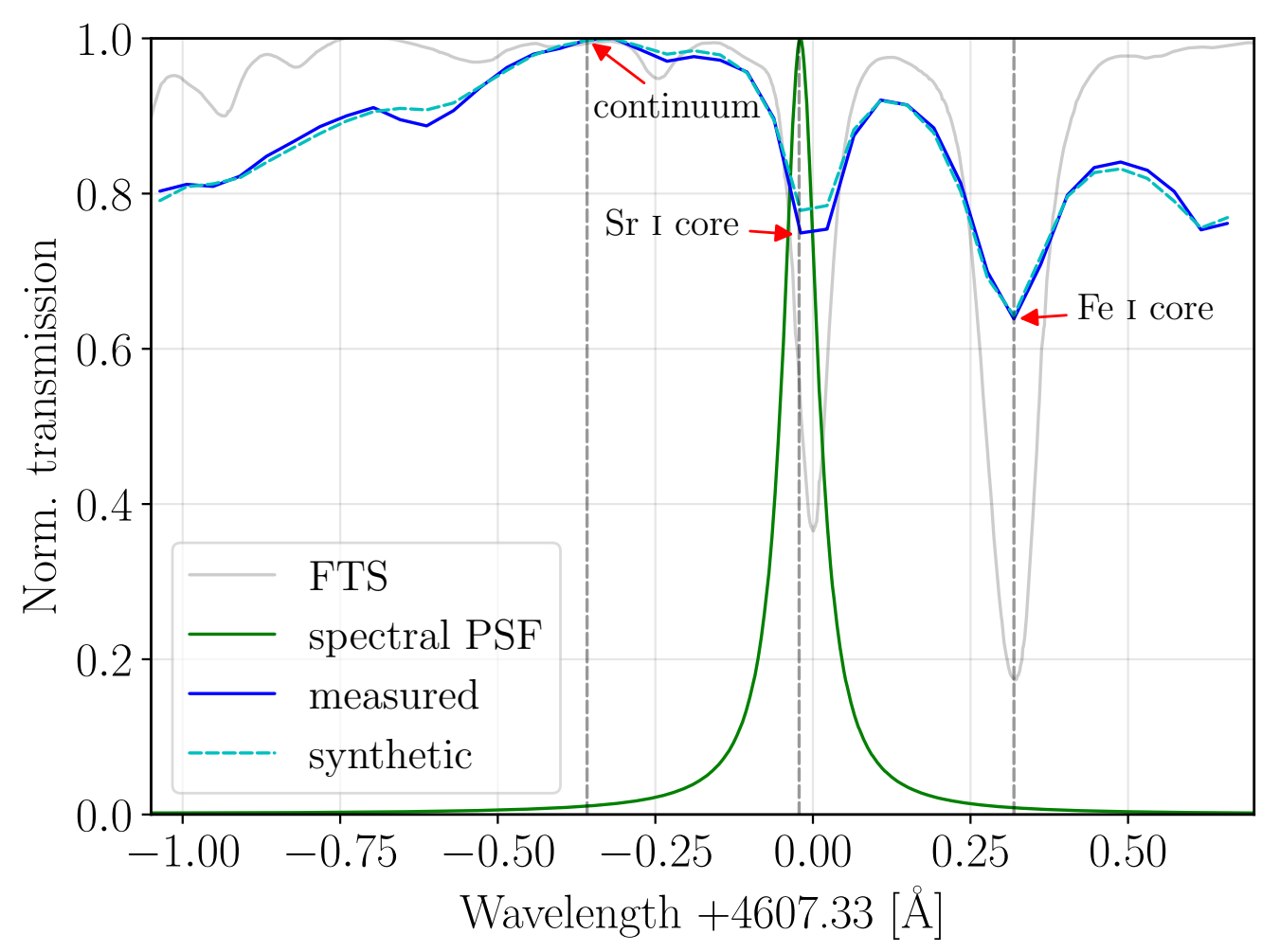

Figure 4.5: Spectral transmission profile (green line) of the filtergraph setup, a measured spectrum (blue) and synthetic spectrum obtained from a convolution of FTS data (Neckel 1999) with the spectral transmission profile. For the measured spectrum the Fabry-Perrot was tuned with a wavelength step of $\sim 8 \mathrm{~m} \AA$. The spectral locations of the observed wavelength positions are marked with arrows.

Note that the sampled wavelength position was coincidentally shifted by about $20 \mathrm{~m} \AA$ into the blue wing of the Sr I line. Note also that we refer to a line core position, but the observation with the broad filter profile returns an integrated spectral line profile. In the Fe I line core position, the observed wavelength position coincides with the nominal line core position very well. At both spectral line core positions we exposed for $210 \mathrm{~s}$, while at the continuum position we exposed for $120 \mathrm{~s}$. The data needed for calibration, i.e., polarization calibration, dark-field and disk center flat-field images are taken within one hour before and after the science data recording.

\subsubsection{Data reduction}

The data reduction steps corrected for dark current, remove common mode and flat-field errors. A polarimetric demodulation is applied. Both channels of the beam-splitter are aligned and fringes are removed. One of the steps takes care of intensity to Stokes $\{Q, U$, $V\}$ cross-talk as well as Stokes $V$ to $\{Q, U\}$ cross-talk. The line core and the continuum images are also corrected. In the following, we will give an overview of these steps in the same order as they are applied. 
In the first step, a low-noise dark image is subtracted. Common mode errors are visible as offsets in pixels belonging to one sensor row due to electrical potential fluctuations in the readout hardware. For the common mode correction, in each frame the signal in 30 shielded pixels for each row on the left and right sides of the sensor are averaged. The average is subtracted from the respective row.

Two hundred and fifty individual frames per modulation state are averaged, which corresponds to $5 \mathrm{~s}$ integration time. With a total of 3.5 and 2 minute observation intervals in the spectral line cores and continuum, 42 and 24 modulation state images, respectively, are obtained per two-beam channel. The flat-field is dark current corrected and common mode corrected. Then the individual images are flat-fielde Although possible, we decided not to apply multi-object, multi-frame blind deconvolution (MOMFBD, see van Noort et al. 2005) restoration to the images to keep the noise distribution as close to the original as possible. The seeing was stable enough to provide high-resolution data without the restoration. To align the two channels of the dual-beam setup, we applied a mapping resulting from aligning reduced dot-target images. The numerical routine for alignment fragments the dot-target images and subaligns these fragments, while rotation, (de)magnification, and horizontal and vertical shifts are taken into account.

To calibrate the data, we used known polarization states generated by the polarization calibration optics at the DST. The polarimetric efficiencies calculated from the modulation matrix after calibration are $0.42,0.59$ and 0.54 for $Q, U$ and $V$, respectively. We applied a rotation matrix around the Poincaré sphere axes $U$ and $Q$ for a heuristic cross-talk correction between $V$ and $\{Q, U\}$ in both channels separately, but field independent (i.e., all pixels in the FoV are rotated with the same angle). With this correction, we take care of residual telescope polarization. The angles of rotation are found by minimizing the cross-talk from the strongest Stokes $V$ signal to the linear polarization images. The estimated cross-talk for $V$ to $Q$ is corrected by $33^{\circ}$ and $28^{\circ}$ rotation around the $U$ axis for the each of the dual-beam channels. In an analogous manner, for a small cross-talk from $V$ to $U$, we corrected with a $-3^{\circ}$ and $-8^{\circ}$ rotation around the $Q$ axis.

Large-scale polarized fringes were visible in polarimetric Stokes $U$ and $V$ Sr I line core images. The polarized fringe removal is done by taking a 2D Fourier transform of the images. A ring-shaped mask is defined and, subsequently, the masked frequencies are removed. The mask is identical for the Stokes $U$ and $V$ images. The removed frequency components are replaced by interpolations from the remaining neighbor pixels and the image is inverse Fourier transformed.

The polarimetric images are then normalized to Stokes $I$ at the corresponding wavelength. After normalization, the expected mean linear polarization signal is very low for an averaged quiet Sun region at disk center in the line core (see Neckel 1999). Therefore, we subtracted the spatial average of $Q / I, U / I$, and $V / I$ from the respective images to correct for residual instrumental polarization. Stokes $Q / I$ is the parameter which suffers the most from an offset of about $1 \%$.

In the last step, we corrected for cross-talk from Stokes $I$ due to sensor nonlinearity. For this correction, it is necessary to determine the factor $f$, followed by subtraction of $f \cdot I$ from the respective normalized polarimetric image. For each polarimetric Stokes $\{Q / I$, $U / I, V / I\}$ image as well as for each time step we determine $f$ separately. To find $f$, we minimized the rms of the image, as more structure from the Stokes I cross-talk will result in a higher RMS value. The largest $f$ coefficients were found for the Stokes $Q / I$ images, 
which is not surprising, as here we found the largest spatially averaged polarimetric offset, indicating a large telescope polarization. Telescope polarization combined with sensor nonlinearity results in significant Stokes $I$ cross-talk. The $f$ values for $U / I$ were at least one order of magnitude smaller than for $Q / I$. Therefore, an increased noise level in Stokes $Q / I$ compared to $U / I$ is due to the ad hoc cross-talk correction from Stokes $I$. Finally, the images of both dual-beam channels are averaged and the mean values of each of the polarization Stokes images are subtracted.

From an azimuthally averaged power spectrum, we estimate a resolution of $0.4^{\prime \prime}$. Based on this estimated resolution we further spatially binned $3 \times 3$ pixel $^{2}$ to a sampling of $0.19^{\prime \prime}$ pixel $^{-1}$.

\subsection{3 $J_{2}^{2}$ maps}

Here we display the complex quadrupole moment of the radiation field $J_{2}^{2}$ in a hypothetical scattering layer estimated from a $5 \mathrm{~s}$ Stokes $I$ image in Figure 4.6. Use was made of equation 4.1 , where the incoming intensity $I(\theta, \chi)$ on the scattering layer depends on the distance $h$ between the scattering layer and the atmosphere radiating at an intensity given by Stokes $I$. The only free parameter of the model to calculate the $J_{2}^{2}$ maps is therefore the height $h$. We varied the parameter $h$ between 1 and 5 spatial pixels, and found that the $J_{2}^{2}$ maps with $h=3$ pixels show the highest contrasts (about $10 \%$ more than the $h=5$ pixel case). The contrast is a proxy for the spatial correlation of the radiation field, which, in turn, determines the structure sizes in the $J_{2}^{2}$ maps. The highest contrast is achieved where the $J_{2}^{2}$ structures are in the order of the correlation length of the underlying radiation field.

We tested if $h=3$ pixels is the best choice by repeating the analysis for all three $h$ cases, and find that the reconstructed polarization signals are almost twice as large for the $h=3$ pixels case than for the other two cases. With our achieved pixel sampling, 3 pixels would correspond to a physical height of about $150 \mathrm{~km}$ above optical depth unity. This is a reasonable number, as Bommier et al. (2005) found a formation height for Sr I between $220 \mathrm{~km}$ and $330 \mathrm{~km}$ at limb distances $\mu=0.55$ and $\mu=0.09$, respectively. Since we observed close to the disk center, we expect to sample deeper layers when observing in the Sr I core. Detailed theoretical investigations by del Pino Alemán et al. (2018) revealed at disk center an average height of formation of the Sr I line of $170 \mathrm{~km}$ above optical depth unity. 

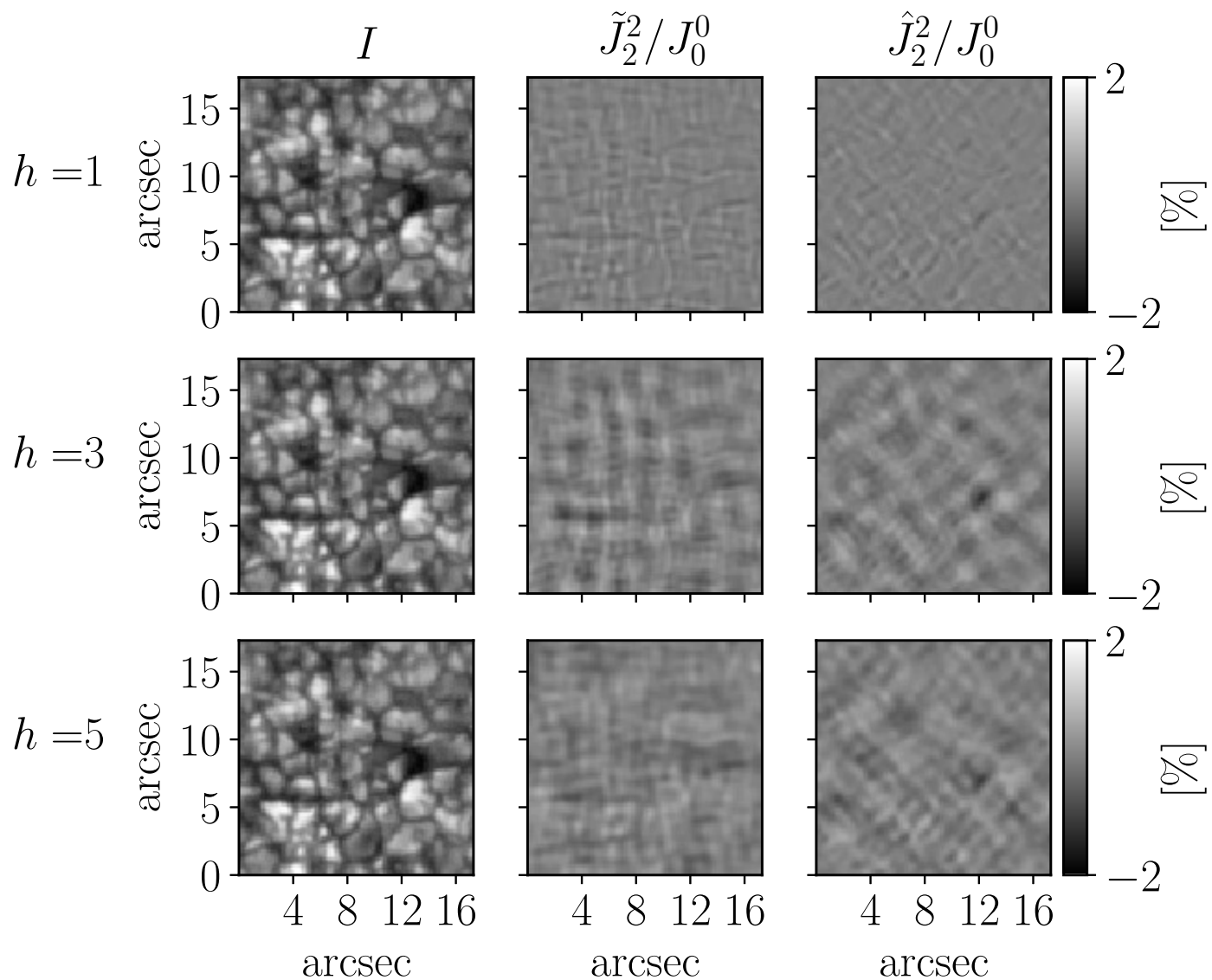

Figure 4.6: Normalized real (center panels) and imaginary (right panels) parts of $J_{2}^{2}$, calculated from the observed ( $5 \mathrm{~s}$ ) intensity Stokes $I$ images (left panels) with equation 4.1. The gray scale on the right of the figure only applies to the normalized $J_{2}^{2}$ maps. The grey scale of the (to the maximum value) normalized Stokes $I$ images is between 0.65 and 0.98 . From top to bottom the parameter $h$ (given in pixels) is increased from 1 to 5 in steps of 2 , which has no effect on the Stokes $I$ image, but influences $J_{2}^{2}$. 


\title{
5 Paper III: Sr I 4607 Å line at solar disk center: a statistical comparison between observation and simulation
}

\author{
in preparation
}

\begin{abstract}
Franziska Zeuner, Tanausú del Pino Alemán, Rafael Manso Sainz, Sami K. Solanki, Javier Trujillo Bueno, Alex Feller

My contribution: Preparing the simulation data for analysis, analysing the data, writing of the manuscript.
\end{abstract}

\subsection{Abstract}

This manuscript aims to compare the scattering polarization in the Sr I line at $4607 \AA$ at solar disk center obtained by an observation with an simulation. The observation of Sr I is reported by Zeuner et al. (2020). del Pino Alemán et al. (2018) calculated Sr I with a detailed 3D radiative transfer calculation in a realistic MHD setup. It is crucial to degrade the simulations carefully to make a fair comparison, as any scattering signal detected by an observation is dependent on the observational setup itself. In our case, this is straight forward for the spectral and spatial resolution. But as the simulation only provides a single snapshot in time, we propose a workaround which provides a hint on how temporal evolution affects the scattering signal amplitude. This workaround is based on a statistical averaging method proposed by Zeuner et al. (2020), which also provides the possibility of comparing the scattering signals in two different solar scenes (as in the simulation and observation). We find that, if temporal evolution is taken into account, the average signal amplitudes predicted by del Pino Alemán et al. (2018) are confirmed by Zeuner et al. (2020). Beyond statistical averaging, Zeuner et al. (2020) proposed to use the statistically averaged data to reconstruct the actual spatial distribution of scattering polarization. The simulation data provides an ideal scenario to test the quality of the scattering polarization reconstruction. We find that the reconstructed scattering polarization distribution follows the actual distribution not perfectly, but quite well. However, the quality of the reconstruction depends on the noise level of the original data. 


\subsection{Introduction}

A major branch in solar physics aims for deducing and understanding the small-scale magnetism, covering at least $99 \%$ of the solar lower atmosphere at any given time. Especially unresolved turbulent magnetic activity in the photosphere is held responsible contributing significantly to the energy balance of our closest star (Trujillo Bueno et al. 2004), while the question regarding the origin or dynamics of magnetic fields operating on scales below the resolution element is still not resolved. The study of scattering polarization signals altered by the Hanle effect opens a window to deduce unresolved magnetic fields on the Sun. One of the strongest scattering polarization signals in the solar photosphere is provided by the Sr I line at $4607 \AA$, which has been widely studied. However, it has been recently discovered that the scattering polarization signal in the Sr I line itself is spatially structured on granular scales at solar disk center (Zeuner et al. 2020). This was theoretically predicted by Trujillo Bueno \& Shchukina (2007) and then synthesized in a realistic 3D-MHD model by del Pino Alemán et al. (2018). To enable the detection of turbulent small-scale magnetic fields, it is necessary to compare the scattering polarization amplitudes in the photosphere from observations with the zero-field case. However, there are many other parameters besides the magnetic field which alter the polarization amplitude: Collisions, the radiation field structure and velocity gradients. The zero-field case can only be provided by solving the 3D radiative transfer problem in a realistic model of the photosphere. However, modeling the photosphere requires constraints of its thermodynamic structure. The information on the thermodynamic structure may be deduced by other spectral lines. We leave this step for future work.

Alternatively, observed scattering amplitudes can be compared with models of the photosphere including a turbulent small-scale magnetic field. One of these models (Rempel 2014 ) provides a magentic field close to equipartition of $\langle B\rangle \approx 170 \mathrm{G}$. Combined with collisional rates for the Sr I line given by Faurobert-Scholl et al. (1995), the synthesized scattering amplitude emerging in this model is consistent with the observed center-tolimb variation of the scattering amplitude (del Pino Alemán et al. 2018). Until recently, scattering polarization signals at the center of the solar disk were unavailable. Thus, the comparison between the center-to-limb variation and the model mentioned above was incomplete. At disk center, the scattering structures have to be resolved in order to be measured (del Pino Alemán et al. 2018; Zeuner et al. 2020). This allows for a detailed examination beyond a simple scattering amplitude comparison of simulation and observation.

In this manuscript we aim to compare disk center theoretical (del Pino Alemán et al. 2018) scattering polarization signals in the Sr I line at $4607 \AA$ with an observation carried out by Zeuner et al. (2020). The simulation includes a small-scale magnetic field with $\langle B\rangle \approx 170 \mathrm{G}$. In contrast to published Sr I data so far, Zeuner et al. (2020) provide high resolution data at disk center with high polarimetric sensitivity. Still, due to instrumental drawbacks, the direct observation of linear polarization patterns in the observed maps was impossible. A new statistical method was used to reveal spatial structures of the scattering polarization on sub-granular scales. The major advantage of this method is that it can be applied to both, simulation and observation. Then, the statistical values can be compared.

As the simulation lacks temporal evolution, we test if any discrepancy between the linear polarization amplitude in the observation and the simulation results from temporal 
averaging.

Another test we conduct is how reliable the scattering amplitudes can be reconstructed after statistical averaging. For this, we compare the scattering amplitudes of the simulation before and after applying the statistical method.

We start by briefly listing the parameters of the observation and the simulation in Section 5.3. The listing includes a description of how the simulation is degraded. We then present in Section 5.4 the results obtained by using a $J_{2}^{2}$-classification based reconstruction of the linear polarization maps proposed by Zeuner et al. (2020). $J_{2}^{2}$ is the quadrupole moment of the radiation field estimated from the intensity map. We use the results to (a) test the reliability of the reconstruction and (b) a quantitative comparison between observation and simulation. Finally, we discuss the implications of the results with respect to future observations and theoretical models. We find that the intermediate step of reconstructing the scattering polarization pattern from a statistical average in the simulation is working remarkably well. This gives us confidence that the observed reconstructed maps imitate the noise-buried structures very well.

\subsection{Observation and simulation data description}

The details of the observation of the Sr I $4607 \AA$ line can be found in Zeuner et al. (2020). The most critical parameters are listed below:

- disk center observation

- original spatial plate scale (critically sampled): $0.062^{\prime \prime} \mathrm{pixel}^{-1}$

- estimated resolution: $\sim 0.4^{\prime \prime}$

- applied $3 \times 3$ spatial binning, therefore final spatial plate scale: $0.2^{\prime \prime}$ pixel $^{-1}$

- spectral resolution determined by single Fabry-Pérot etalon and pre-filter: full-width at half-maximum (FWHM) is $67 \mathrm{~m} \AA$, free spectral range is $1.75 \AA$, the pre-filter FWHM is $169.7 \mathrm{~m} \AA$ (profile displayed in Zeuner et al. 2020)

- observed wavelength positions: Sr I, Fe I and a continuum position (in the following, we present the analysis for the $\mathrm{Sr}_{\mathrm{I}}$ although we did the analysis alike for the continuum)

- the size of analyzed FoV is around the lockpoint of the AO and about $17.3^{\prime \prime} \times 17.3^{\prime \prime}$

- duration of observation in Sr I: 3.5 min, $5 \mathrm{~s}$ cadence (but recorded at 200 frames s${ }^{-1}$ and $50 \mathrm{~Hz}$ modulation to minimize seeing-inuced cross-talk)

- final noise level in temporally averaged images of $\sim 0.04 \%$ in linear polarization (slightly higher in $Q / I$ than $U / I$ due to an ad-hoc cross-talk correction)

The (temporally averaged) observed linear polarization signals and intensity in Sr I can be seen in Figure 5.1 in the top three panels (a)-c)). Besides the low noise level, no obvious spatial structure with respect to the intensity image is visible, i.e., the polarization images are noise dominated. This data set is referred to as obs.. The details about the 3D-MHD 
simulation and the synthesis of the Sr I $4607 \AA$ with the PORTA code can be found in del Pino Alemán et al. (2018). The important parameters are:

- original resolution: $8 \mathrm{~km}$

- spectral sampling: $3.6 \mathrm{~m} \AA$

- size of FoV is $6144 \mathrm{~km} \times 6144 \mathrm{~km}$

- single snapshot in time

The simulation was degraded to the observation in the following way:

a) convolve spectrally with spectral PSF at Sr I position of the observation

b) normalize $Q, U$ with $I$

c) re-sample simulation to observation (original spatial sampling, with cubic interpolation)

d) degrade additionally by applying Gaussian filter of width 2.4 pixels to imitate resolution of $0.4^{\prime \prime}$

e) $3 \times 3$ spatial binning

f) extended the FoV according to observation by wrapping the images (to leave the original spatial symmetry uninfluenced)

g) apply Gaussian noise to polarization maps according to the observation

h) later, before the polarization pixels are averaged based on the $J_{2}^{2}$-maps (the maps which are calculated from the intensity image), we add Gaussian noise to the normalized $J_{2}^{2}$-maps with standard deviation of 0.05 (equals the step size of polarization binning within $J_{2}^{2}$ )

We show the simulated intensity and linear polarization signals in Figure 5.1 in the panels f-h). This data set is referred to as $\mathrm{sim}$. We use another version of this data set later where we did not apply step g), and therefore is noise-free. The polarization amplitude is reduced by about a factor of 10 compared to the undegraded simulation, especially when a spectral filter is applied. The undegraded simulation is not shown here but can be found in del Pino Alemán et al. (2018). Most notably, after applying the noise, the signals are buried in the noise and therefore simulations reproduce non-observable linear polarization signals with the observational setup.

Step h) was carried out to make sure that the artificially extended FoV has the same number of distinct pixels as the observation. It also demonstrates that the reconstruction is robust against extra noise in the $J_{2}^{2}$ maps. The idea behind this step is the following: We aim to compare the simulation with the observation as fair as possible. The observational FoV can not be cropped, because then the number of pixels is too low to reach a sufficient signal-to-noise ratio. If the FoV of the simulation is extended, the pixels exist multiple times, and the number of distinct $J_{2}^{2}$ values is exactly the same. Therefore, during averaging, the noise reduces differently to the observation. As a test, we did the analysis without this step, but especially a) and b) of Figure 5.2 look less similar to the observation. In particular, the $\hat{J}_{2}^{2}-\tilde{J}_{2}^{2}$ plane is less filled. 

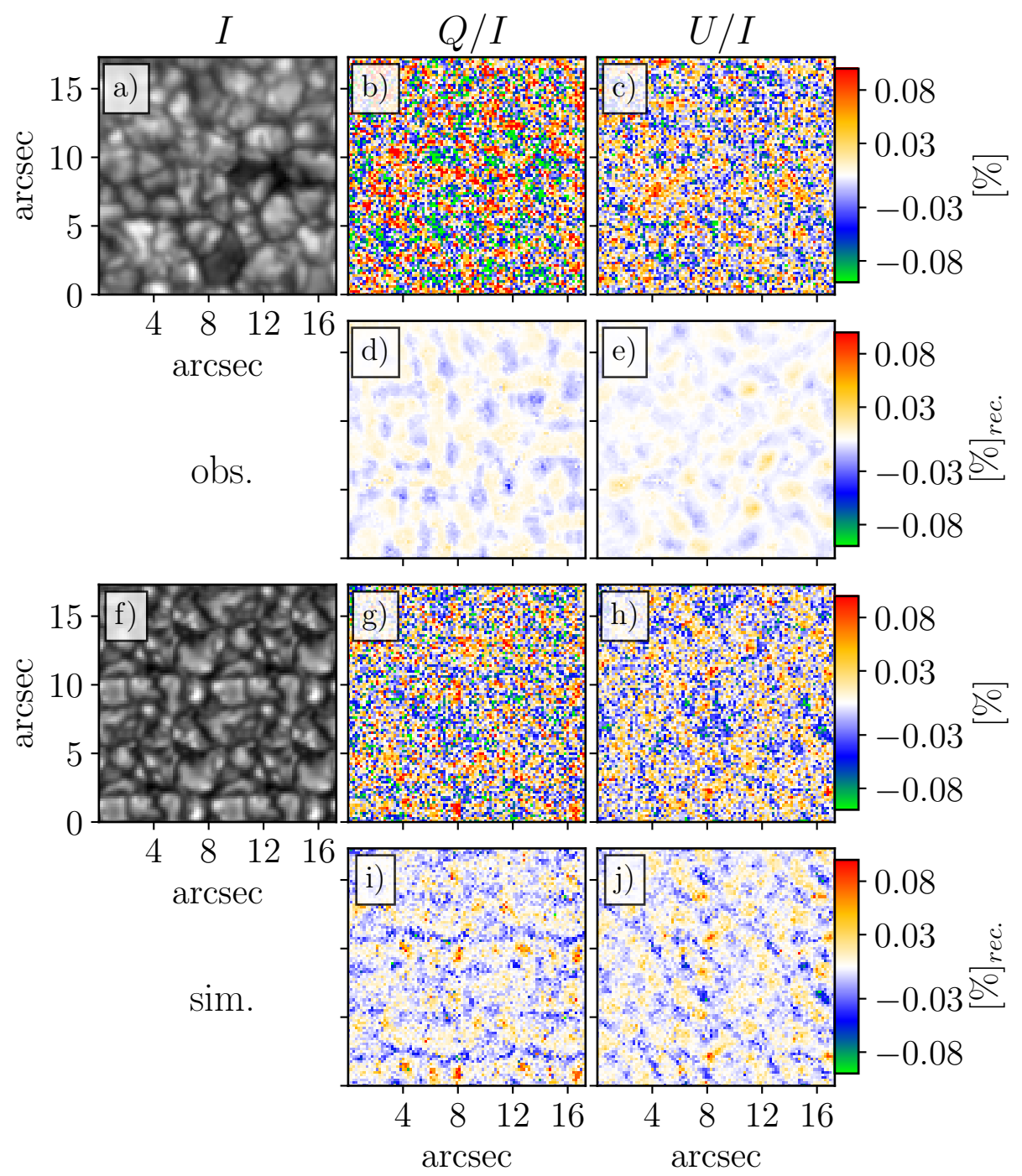

Figure 5.1: Observed and simulated Sr I Stokes $I, Q / I, U / I$ maps and corresponding reconstructed linear polarization maps. a) Observed, temporally averaged intensity image and observed $Q / I$ and $U / I, \mathrm{~b}$ ) and c), respectively. d-e) Reconstructed $Q / I$ and $U / I$ spatial maps from the statistical averaged polarization in panels e-f) of Figure 5.2. The statistical average is based on the $J_{2}^{2}$ values (see text for details). These maps have been reconstructed by retracing the accumulated polarization values from Figure 5.2 to their original positions in an observed $5 \mathrm{~s}$ image (i.e., to the places with the corresponding $J_{2}^{2}$ values) and then temporally averaged. f) Degraded single-snapshot theoretical intensity image and Stokes $Q / I$ and $U / I$ images, g) and $\mathrm{h}$ ), respectively. i) and j) The exact same method as for the observed Stokes images is used to reconstruct the spatial distribution of $Q / I$ and $U / I$ from the statistical averaged polarization in panels a-b) of Figure 5.2. Note that the signals in the simulation is higher, where temporal evolution is neglected and that the intensity images are dominated by the continuum due to the broad pre-filter used in the observation. 


\subsection{Results}

In this section we present the results of our analysis with focus on three topics:

1. quantitative comparison between simulated and observed averaged polarization signals based on the respective $J_{2}^{2}$ classification maps

2. comparison of reconstructed polarization distribution with the original distribution, both given by the simulation

3. the role of solar temporal evolution regarding the polarization amplitude

In this section we use a statistical method proposed and described in more detail by Zeuner et al. (2020). The main idea behind the method is to enhance the signal-to-noise ratio by spatially (and, in the case of the observation, temporally) averaging pixels which are exposed to similar radiation asymmetries. To ensure the signals add up coherently, we classify them according to the axial symmetry break of the radiation field, quantified by the quadrupole moment of the radiation field, $J_{2}^{2}$. The quadrupole moment is estimated using the Stokes $I$ intensity image. In the particular case of the observation made by Zeuner et al. (2020), the intensity was dominated by the continuum, due to the broad pre-filter combined with a single Fabry-Pérot etalon. The quadrupole moment is estimated by calculating the complex $J_{2}^{2}=\hat{J}_{2}^{2}+\mathrm{i} \tilde{J}_{2}^{2}$ radiation tensor element from the Stokes $I$ image and then averaging the polarization values within pixels which ensue similar $J_{2}^{2}$ values. At disk center, $Q / I+\mathrm{i} U / I \propto \hat{J}_{2}^{2}+\mathrm{i} \tilde{J}_{2}^{2}$ (Zeuner et al. 2020; del Pino Alemán et al. 2018), if the vertical magnetic field component is not dominant. This allows for noise reduction while the polarimetric signal is conserved. With the averaged values of the polarization, the spatial distribution can be reconstructed (Zeuner et al. 2020).

\subsubsection{Quantitative comparison between simulation and observation}

To make a quantitative comparison between the simulation's polarization signals and the observed polarization signals, the linear polarization as a function of the estimated radiations's quadrupolar component $J_{2}^{2}$ is plotted. As the quadrupolar component is normalized to the maximum value, the resultant averaged polarization maps are independent of the particular quiet solar scene on which the $J_{2}^{2}$-maps are based, see Figure 5.2. Therefore, it is possible to compare the simulation and observation quantitatively, without the bias of the different solar scenes which underlie them.

We show the results obtained by applying the averaged polarization maps with respect to $J_{2}^{2}$ to the observed and simulated data. The linear polarization values are displayed with respect to the real and imaginary part of $J_{2}^{2}$ for the observation (panels e) and f)) and simulation (panels a) and b)) in Figure 5.2.

We find a strong anti-correlation of the linear polarization signals with respect to $J_{2}^{2}$, in the observation as well as in the simulation. More precisely, Stokes $Q / I$ is anti-correlating with the $\hat{J}_{2}^{2}$, while $U / I$ is strongly anti-correlated with $\tilde{J}_{2}^{2}$.

We quantitatively investigate the difference of the observed signals with the simulated signals by fitting a weighted two-dimensional surface to the signals in Figure 5.2. See panels c)-d) for the simulation and g)-h) for the observation for the fitted surfaces and the fitted coefficients. 
We find that the coefficients from the fitting are (on average) almost two-times larger in the simulation than in the observation. The origin of this discrepancy can be manifold. Firstly, it may be the result of the lack of temporal evolution in the simulations and/or higher turbulent magnetic activity in the observed region than in the simulated region, as the Hanle effect also causes depolarization. The observed FoV was not exclusively exhibiting the very quiet Sun, but the observed Stokes V/I (see Zeuner et al. 2020) indicate a higher magnetic field concentration within the FoV of indeterminable strength. Therefore, the variability of magnetic field strengths and resulting depolarization rates may be higher than assumed in the simulation and therefore not represented in the quiet Sun simulation data. Another important point is that the spectral PSF of the observation is much broader than the wavelength range provided in the simulation, leaving the contribution of spectral stray light from the far wings of the PSF unknown. Potentially, stray-light can result in a lower polarization amplitude. Finally, collision rates assumed for the simulation data have been chosen to match the low-resolution center-to-limb variation of the Sr I linear polarization (del Pino Alemán et al. 2018), but may differ in spatially resolved observations. Additionally, the collision rates of del Pino Alemán et al. (2018) could be compared to not more than $\mu=0.6$, as published observations of $Q / I$ closer to disk center proir to 2018 have been missing.

The orientation of the red-blue pattern in Sr I of Figure 5.2 is almost perfectly aligned with the $\hat{J}_{2}^{2}$ and $\tilde{J}_{2}^{2}$ axes. This applies for the observation and the simulation. This means, that no oriented magnetic field with the line-of-sight is present in either the observation or the simulation, as this would rotate the red-blue pattern in the $J_{2}^{2}$-plane due to the Hanle effect. The small rotation in the observation of about $4.5^{\circ}$ is within observational uncertainties caused by the noise ( $Q / I$ has a higher noise level, see previous section).

Because of the strong anti-correlation of the linear polarization signals with the local radiation field in Sr I and the absence or barely visible correlation in the continuum, Zeuner et al. (2020) concluded that the found signals are due to scattering polarization. The scattering signals are structured with respect to the axial symmetry break of the radiation field, quantified by $J_{2}^{2}$. This has also been found by del Pino Alemán et al. (2018), who moreover neglected the Zeeman effect and therefore the Zeeman effect can be excluded as a possible source for the polarization signals in the simulation data.

As a last step, we reconstruct the spatial linear polarization maps using Figure 5.2 (Zeuner et al. 2020). In doing so, the individual positions of the averaged pixels have been remembered and the average value placed back to the individual positions. The resultant image is shown in the panels d)-e) and i)-j) of Figure 5.1 for the observation and simulation, respectively. We find that the reconstructed maps from the simulation data restores most of the noise-buried features in the original Stokes image.

In general, the size and distribution of the spatial structures of the reconstructed polarization are very similar in the simulation and the observation. This is expected, as the observation as well as the simulation are structured with respect to the underlying radiation field. However, as already stated above, the observed polarization show less contrast than the simulated polarization. This is maybe due to the magnetic field or temporal evolution effects, since the observation is reconstructed from $3.5 \mathrm{~min}$ of data while the simulation consists of a single snapshot in time. 
$\operatorname{sim}$.
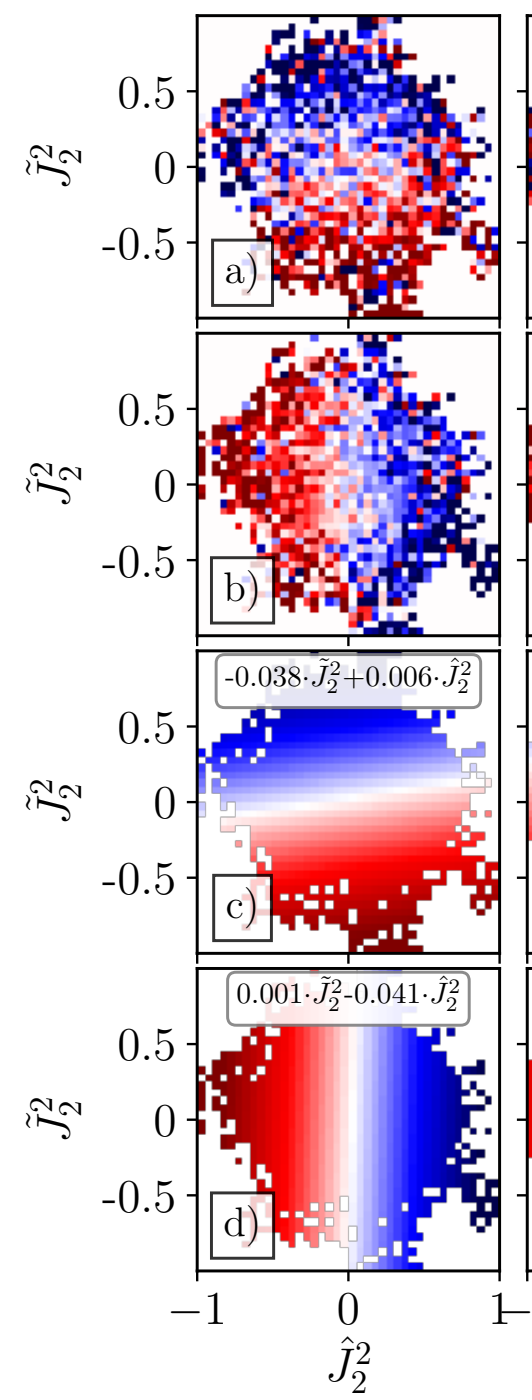

obs.
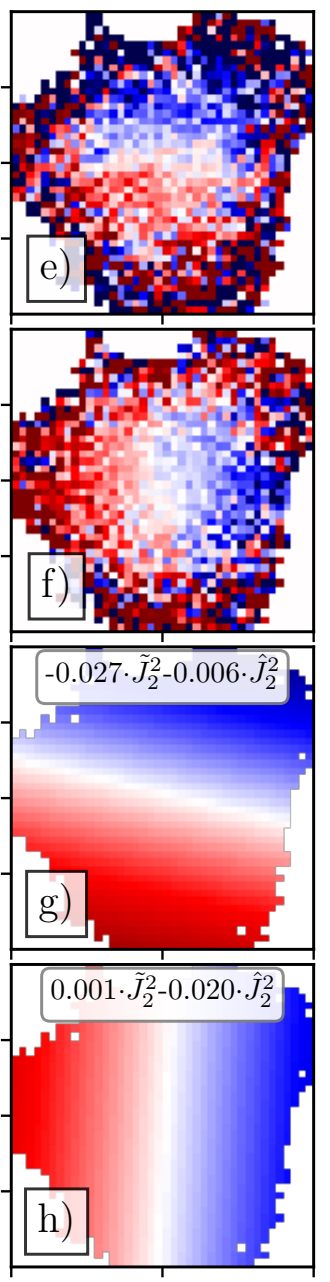

$\begin{array}{lll}-1 & 0 & 1\end{array}$ art. obs.
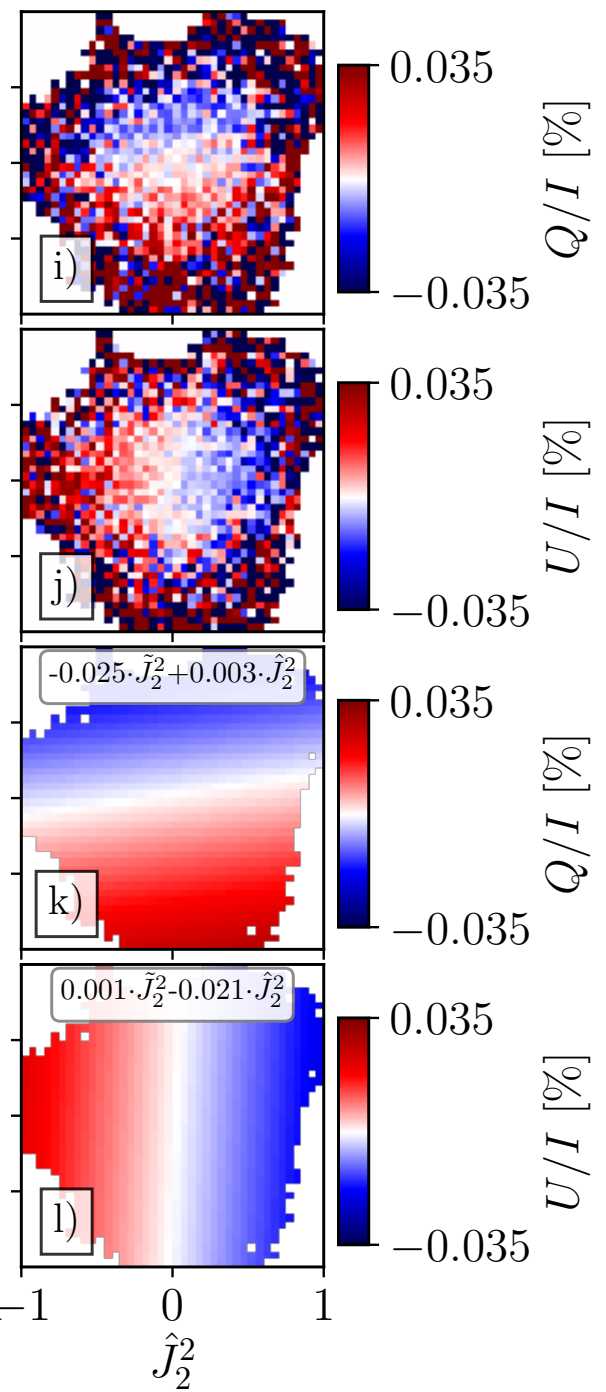

Figure 5.2: Average linear polarization as a function of the estimated radiation tensor element $J_{2}^{2}$ for Sr I of the Stokes images a)-b) for the simulation (sim.) and e)-f) for the observation (obs.). The averages are based on the panels $\mathrm{g}$ )-h) and b)-c) in Figure 5.1). The details of how these panels are generated can be found in Zeuner et al. (2020). The important advantage is that the noise level is significantly lower than in the Stokes images in Figure 5.1 because of averaging. To circumvent spatial smearing of the signals while decreasing the noise level significantly in the observation, the averaging is done with respect to the axial symmetry break of the radiation field in a small time interval. Since for the simulation there is a single snapshot in time, we created an artificial observation (art. obs.). This artificial observation uses the fitted coefficients in the simulation and the observed $J_{2}^{2}$ values, see equations 5.1 and 5.2 and text for more details. For each Stokes $Q / I$ and $U / I$, the two-dimensional surface fit is shown in the six lower panels. 


\subsubsection{Reliability of reconstruction}

To test the quality of the reconstruction, we applied three tests. First, we cross-correlate the reconstructed maps of the observation with the directly observed polarization maps. Secondly, we compare the polarization maps provided by the simulation and the reconstruction based on simulated noise-free and noisy polarization signals. Thirdly, we test the limits of the reconstruction method.

To gain confidence in the pattern seen in the reconstructed maps from the observation, we spatially cross-correlate the observed (obs.) polarization maps and the reconstructed maps (obs., rec.) from Figure 5.1). The cross-correlation for each pair of reconstructed and observed polarization map are presented in Figure 5.3. We find that the largest crosscorrelation is found in the center (zero shift) of the respective reconstructed and observed polarization maps. This indicates that, although not visible by the eye, patterns in the reconstructed maps in the panels d)-e) of Figure 5.1 do have a counterpart in the noise dominated observed Stokes images b)-c).

If we compare the simulated, but noise-free Stokes images (which we plot in the top panels of (a)) in Figure 5.5) with the reconstructed polarization maps (center panels of Figure 5.5), the reconstructed polarization patterns follow the original observed patterns well. Especially this is true if the polarization signals are spatially large and have a large amplitude. However, small-scale details are missing. In Figure 5.4, we plot the difference between the simulation (deg. in Figure 5.5) and the reconstructed simulation (deg., rec. in Figure 5.5) of the linear polarization signals.

We quantified spatial differences by calculating the power spectrum for both, the original and the reconstructed map of the simulation. We find up to $10 \times$ more power at scales between $1.2^{\prime \prime}-2^{\prime \prime}$ for the original map. Therefore, the reconstruction works more reliable for spatially larger signals. In addition, we find that the edges do not show more differences than the center. This gives us confidence that the chosen boundary conditions for estimating $J_{2}^{2}$ are working well and do not introduce systematic errors. Interestingly, some areas show significantly reduced signal amplitudes. Either, depolarization due to stronger magnetic fields are present in these regions which contribute to the reconstructed low-signal areas or that there are additional polarizing mechanisms with opposite sign, such as velocity gradients, which are not considered.

In numbers, the mean values of the polarization amplitude reduce by almost $50 \%$ when reconstructed (compare deg. with deg., rec. of Figure 5.5). It is expected that the amplitudes decrease because of the averaging process.

With the next test we quantify the quality of the reconstruction. For the test, we identified pixels above a certain absolute threshold $(0.02 \%)$ in the noise-free polarization maps provided by the simulation (see contours in panels a) and b), see Figure 5.5). As stated above, small-scales are not very well reconstructed, therefore we exclude them from the analysis. The same contours are reproduced in the reconstruction of the polarization for the noise-free case (panels c) and d) of Figure 5.5) and the noise-included case (panels e) and f) of Figure 5.5). The latter is a sub-sample of panels i) and j) of Figure 5.1. We scatter the polarization values of the reconstructed and contoured pixels with the contoured pixels of the original polarization in (b) Figure 5.5. The scatter is fitted with a linear regression, and the slope has a value of around 0.6 , which shows that the polarization amplitude during reconstruction is systematically reduced by a factor of two. The scatter is higher in the 

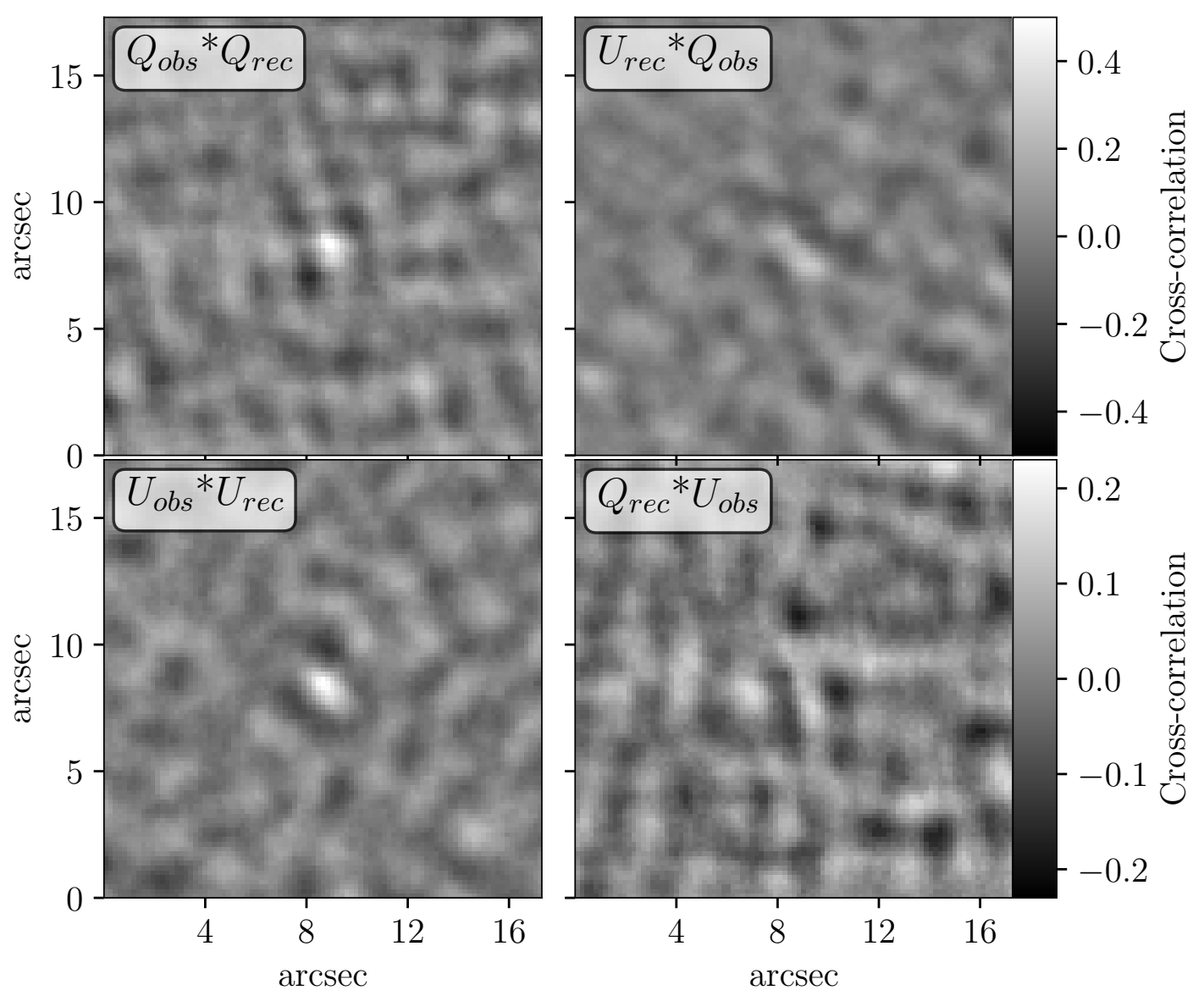

Figure 5.3: Cross-correlation of the observed polarization images with the reconstructed polarization maps. The largest cross-correlation is found where the Stokes parameter of observation matches the Stokes parameter of the reconstruction and with zero offset between observed polarization image and reconstructed map.

noisy case, but the slope is independent of the noise. If simulation and observation are compared, the systematic error apply for both of them. Therefore, statistically they can be compared when using the statistical averages. Remarkably, only very few pixels change the sign during the reconstruction process. About $6 \%$ of the pixels (inside the contour) for the noise-free case and $20 \%$ in the noisy case suffer from sign changes. This means that post-reconstruction spatial averaging should be avoided, since the scattering signal would decrease disproportionately.

\subsubsection{Influence of solar temporal evolution}

As has been mentioned above, the polarization amplitudes of the observation and simulation do not match well, even if the simulation is spatially and spectrally degraded properly. One of our suspicion is that the solar temporal evolution might play a significant role in the degradation of the amplitude. As there is no temporally resolved simulation available yet, we would like to test this hypothesis by assuming that for each time step of the observation the polarization pattern is given by the fitted surfaces in Figure $5.2 \mathrm{c}$ ) and d). We create an 


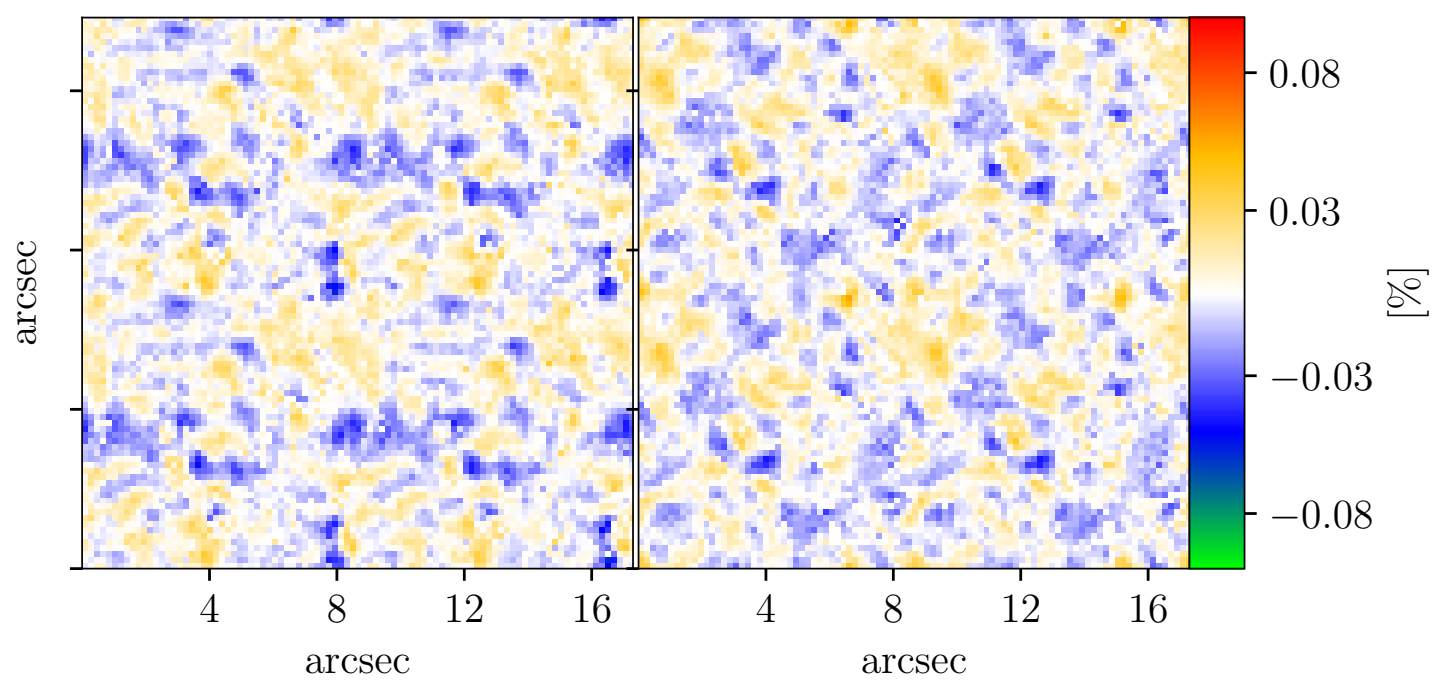

Figure 5.4: Difference images of the noise-free Stokes images and their respective reconstructed $Q / I$ (left) and $U / I$ (right) of the simulation (center panels of Figure 5.5 (a) are subtracted from the top panels). The mean difference is zero, the standard deviation is $0.01 \%$.

artificial observation with scattering signals $(Q / I)_{\text {art. }}$ and $(U / I)_{\text {art. }}$ by using:

$$
(Q / I)_{\text {art. }}=\alpha_{Q, \text { sim. }} \cdot\left(\tilde{J}_{2}^{2}\right)_{o b s .}+\beta_{Q, \text { sim. }}\left(\hat{J}_{2}^{2}\right)_{o b s .}
$$

and

$$
(U / I)_{\text {art. }}=\alpha_{U, \text { sim. }} \cdot\left(\tilde{J}_{2}^{2}\right)_{o b s .}+\beta_{U, \text { sim. }}\left(\hat{J}_{2}^{2}\right)_{\text {obs. }}
$$

for each time step. The coefficients of the simulation are taken from Figure 5.2: $\alpha_{Q \text {,sim. }}=0.039$, $\beta_{Q, \text { sim. }}=0.001, \alpha_{U, \text { sim. }}=0.0, \beta_{U, \text { sim. }}=0.041$. We then applied exactly the same steps for getting Figure 5.2. The results are displayed in i)-1) of Figure 5.2.

The most remarkable result is that, even when we started with coefficients as high as $\beta_{U, \text { sim. }}=0.041$, the coefficients reduced by almost a factor of two after $3.5 \mathrm{~min}$ (i.e., $\beta_{U, \text { art }}=0.021$, see Figure 5.2 panels $\mathrm{k}$ ) and 1)). The values are remarkably close to the observed coefficients (see Figure 5.2). We do not show the reconstructed linear polarization maps but we find that they look very similar to the observed reconstructed maps in Figure 5.1. This emphasizes the strong connection between the polarization and the radiation field, which in turn depends on the granulation. It is therefore not surprising that the typical life time of granules, which lies in the order of minutes (Hirzberger et al. 1999), reflects in the life time of the scattering signals. 


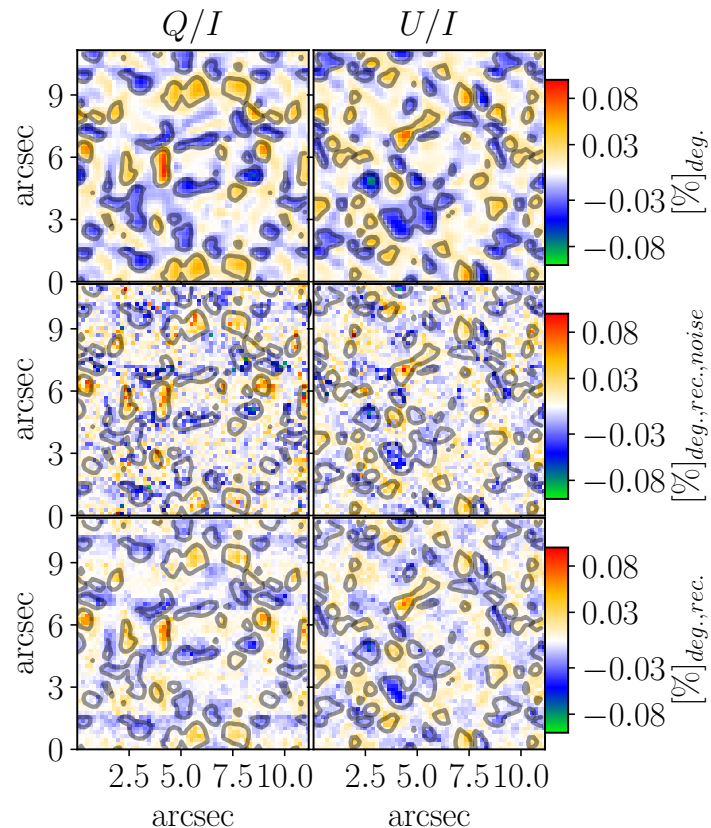

(a)

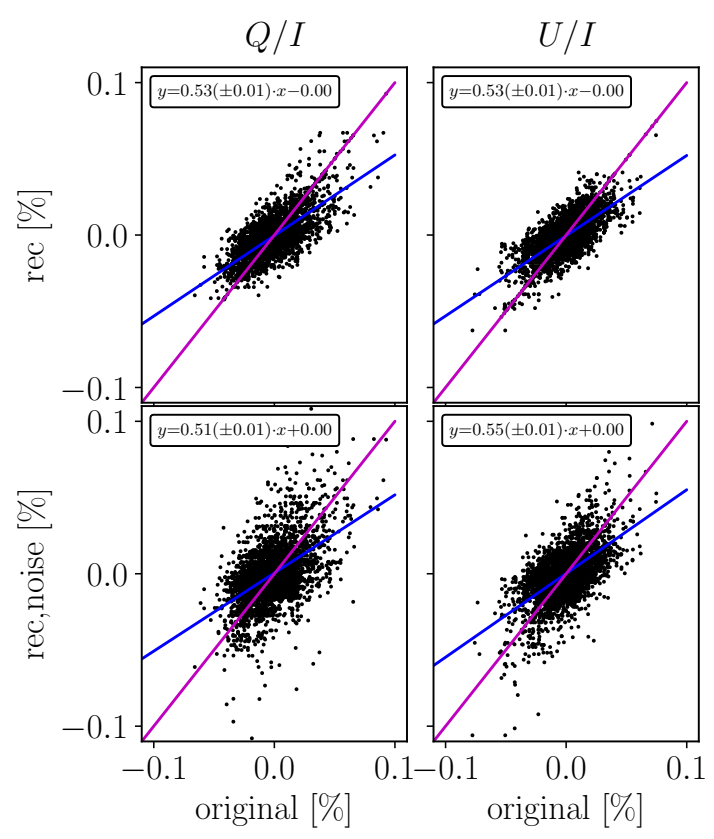

(b)

Figure 5.5: (a) Sub-sample of the simulation with contours around the original theoretical signals. Top panels: noise-free simulated polarization map with contours at the threshold level of $0.02 \%$. The contours are repeated in the reconstructed polarization maps, without (bottom panels) and and with added noise (center panels), therefore based on the images in the panels g) and h) of Figure 5.1) in the original theoretical Stokes images. (b) Scatter plot between simulated noise-free polarization pixels above an absolute threshold of $0.02 \%$ with the reconstruction of the same pixels. The scatter is fitted by a linear regression, where we give the fitted line in the inset. The slope for the magenta line is one.

\subsection{Discussion and conclusion}

For the first time we compared a spatially resolved disk-center scattering polarization observation with a realistically simulated scattering polarization signals. The motivation is two-fold: on the one hand, the method of reconstructing spatial scattering maps via a $J_{2}^{2}$-classification can be tested with simulated polarization signals, while the observation provide the first evidence of predicted scattering patterns by s simulations. It has been shown already by del Pino Alemán et al. (2018) and Zeuner et al. (2020) that the scattering polarization is well correlated with the illuminating radiation field. Missing was how the simulated signals will change when the classification prior to reconstruction is used. We find that the overall spatial distribution is well reconstructed, given that the original maps are too noisy to reveal any spatial pattern. It is also important to note that the polarization amplitude is not enhanced but rather reduced during the reconstruction process. Therefore, any quantitative analysis based on reconstructed maps will give upper limits for the scattering polarization amplitude. We showed that for the observation the reconstructed maps are correlated with the original (but too noisy) polarization maps. From this we conclude that the patterns seen in the reconstructed map of the observation are significant. 
Although simple and useful, the underlying assumption, that the scattering polarization is purely determined by the radiation field, is of course incomplete. del Pino Alemán et al. (2018) show that (especially horizontal) macroscopic velocities also play a very significant role in the scattering polarization of $\mathrm{Sr} \mathrm{I}$, as they add asymmetries to the environment and create polarization. The role of collisions, which might also be important since the Sr I line forms in the photosphere where collisions are still significant (Bommier et al. 2005), is hard to quantify with the observation. However, del Pino Alemán et al. (2018) used collisional rates well represented by center-to-limb data in the Sr I line. Another important ingredient to modify polarization signals is the magnetic field. This aspect is not yet studied in this manuscript.

The results so far can be summarized as follows:

- Scattering polarization from synthesized Sr I in a realistic 3D-MHD simulation is reconstructed well using the method explained in Zeuner et al. (2020).

- Larger and stronger structures ( $>1^{\prime \prime},>0.05 \%$ scattering amplitude) are better reconstructed than weak and smaller structures. The estimates of the spatial structure given in Zeuner et al. (2020) $\left(0.75^{\prime \prime}-1^{\prime \prime}\right)$ may therefore be biased by the reconstruction method.

- The reconstruction method introduces a systematic 50\% decrease in the polarization amplitude and about $6 \%$ error in the pixel sign compared to the original polarization distribution.

- The polarization distribution is similar in the simulation and observation.

- The simulation has two times more signal amplitude than the observation.

- The temporal evolution of the granulation result in a decrease of temporally averaged linear polarization amplitudes, which is in the order of the discrepancy between the amplitude predicted by the simulation compared to the observation (see previous item). 



\section{Summary and outlook}

In this thesis high resolution spectropolarimetry observations of the Sr I spectral line, located at $4607 \AA$, was investigated. Specifically the spatial structure of Sr I scattering polarization in the magnetically quiet solar photosphere was examined.

Zeuner et al. (2018) analyzed the first filtergraph observation of scattering polarization in $\mathrm{Sr}$ I at a limb distance of $\mu=0.6$. In contrast to published spectrograph observations (e.g., Dhara et al. 2019), it was found that the amplitude of scattering polarization is significantly anti-correlated with the continuum intensity. Such results indicate that more scattering polarization is present towards the intergranular lanes than in the center of the granules. This supports the theoretical prediction by Trujillo Bueno \& Shchukina (2007) and del Pino Alemán et al. (2018). They predicted that the local structure of the scattering polarization in Sr I is determined by the axial symmetry break of the radiation field. The symmetry break of the local radiation field is expected to be strongest at the interface between granules and intergranules, where cool and hot plasma meet. Nevertheless, the discrepancy in Sr I observations between spectrograph and filtergraph remains unsolved.

Two important aspects of spatially structured scattering polarization in the Sr I line may contribute to the discrepancy. On the one hand, the structuring occurs on small-scales of the order of $0.5^{\prime \prime}-1^{\prime \prime}$ (Zeuner et al. 2018, 2020). On the other hand, the time scale on which the granulation changes is relatively short, of the order of minutes. Consequentially, the local radiation field changes on a similar time scale. As demonstrated by Zeuner et al. (2020b, in prep.), larger cadences in observations thus affect the amplitude of the observed scattering polarization. Therefore, spectrograph observations, with low resolution and long integration time, may not be able to capture the spatial structure determined by the local radiation field.

It is expected that the correlation between the scattering polarization and the local radiation field is most noticeable when observed at disk center. In light of that, the interest in observing the linear polarization in Sr I at solar disk center increased in the past decade. In an observation of the solar disk center with a spectrograph with an integration time of about 6 minutes, analyzable linear polarization signals in Sr I were absent (Dhara et al. 2019).

Zeuner et al. (2020) reports on a novel high-cadence, high resolution spectropolarimetric observation of a disk center quiet Sun region. The observation was obtained with a filtergraph. The region was observed for 3.5 minutes. Structured scattering polarization with respect to the local radiation field is found for the first time at disk center. Further, more scattering polarization is found closer to intergranules, confirming the findings of Zeuner et al. (2018) and the theoretical predictions (del Pino Alemán et al. 2018). The results are based on a novel pixel-classification method. They reveal that the polarization is induced by a symmetry break of the local radiation field and is therefore highly dependent 
on the concurrent thermodynamic environment. Typically, in order for the scattering signals to emerge above the photon noise level, exposure times of minutes or even hours are required. With increasing exposure times the risk of spatial smearing increases, as solar evolution in the lower layers progresses with characteristic time scales of minutes. The novel pixel-classification method allows for reducing the photon noise significantly. Additionally, the spatial information is moderately conserved. The observed scattering polarization amplitude is on the order of $4 \cdot 10^{-5} I$. The estimated spatial scale of about $0.75^{\prime \prime}-1^{\prime \prime}$ refines the scales estimated in Zeuner et al. (2018). Reconstructed scattering polarization maps are provided. Nevertheless, a direct measurement is missing.

Further, it is found that the observation is consistent with a small-scale turbulent magnetic field in the lower solar atmosphere. A magnetic field with a strong residual component in the vertical direction can be ruled out with a high degree of certainty. This conclusion was based on the absence of an angle between the polarization plane and the radiation field. Usually, the presence of an angle is a signature for Hanle rotation. However, the angle in the observation was small enough to be explained entirely by noise effects.

To quantitatively compare the theoretically predicted scattering polarization amplitudes (del Pino Alemán et al. 2018) with the observation, the simulation data have to be spectrally and spatially degraded to match the observation. This is important, since the spectral and spatial resolution affect the scattering polarization amplitudes. Zeuner et al. (2020b, in prep.) aim to achieve a fair comparison between the simulation and the observation.

In Zeuner et al. (2020b, in prep.), the technique developed in Zeuner et al. (2020) is applied to a detailed 3D radiative transfer calculation of the Sr I scattering polarization by del Pino Alemán et al. (2018). The radiative transfer calculation was conducted in a realistic 3D MHD snapshot of the quiet solar photosphere. After careful spectral and spatial degradation of the simulation data to the level of the observation in Zeuner et al. (2020), it is found that the scattering polarization amplitudes of the observation and simulation differ by a factor of about two. To test if temporal evolution may be responsible for the discrepancy, further analysis has been carried out. The analysis indicates that the temporal evolution of the solar atmosphere induce a decrease of the scattering polarization amplitude. Such a decrease can adequately explain the missing factor of two. It is expected that the temporal evolution of the granulation influences the scattering polarization in Sr I for the following reason: Kinematically driven changes of the solar atmosphere potentially produce scattering polarization with opposite signs. Thus, the scattering polarization signals may cancel when temporally averaged. A similar conclusion has been drawn in Appendix C.2 from the observational data. del Pino Alemán et al. (2018) stated that high-cadence observations will be crucial to observe scattering polarization fluctuations on small scales. Nevertheless, so far simulations of the scattering polarization in $\mathrm{Sr}$ I lack temporal evolution. Therefore, a theoretical prediction on the lifetime of scattering polarization is missing.

The novel statistical method proposed by Zeuner et al. (2020) has the advantage that it is applicable to both, simulations and observations. The method allows to compare simulations and observations of the Sr I scattering polarization statistically. The possibility to compare scattering polarization amplitudes provided by simulations and observations, independently of the particular solar scene, is a prerequisite for magnetic field inferences from the Hanle effect. However, to extract information about magnetic fields based on the Hanle effect, new methods have to be developed. For example, not only the magnetic field, 
but also other sources and sinks of scattering polarization in Sr I must be unambiguously identified.

This thesis contributed to identify one important source of Sr I scattering polarization, which is the symmetry break of the local radiation field. Given that the radiation field in the photosphere is structured with respect to the granulation, the Sr I scattering polarization itself is spatially structured. The importance of measuring spatially structured scattering polarization is in the potential to infer unprecedented information about unresolved, weak and small-scale turbulent magnetic fields permeating the lower solar atmosphere.

The results of this thesis have implications on both, future solar observations and modeling spectral line scattering polarization in realistic magnetohydrodynamic simulations of the Sun. Thereby, some promising and exciting possible future work includes:

- Exploit the power of the newly operating large-aperture Daniel K. Inouye Solar Telescope (DKIST, operated by NSO) on Hawaii for small-scale scattering in spectral lines, especially with good spectral resolution. Fixing the spatial resolution of DKIST to the diffraction limit of current $1 \mathrm{~m}$-class solar telescopes, which is about $0.1^{\prime \prime}$, DKIST will provide a significantly increased photon flux of more than a factor 10. This will help increase the spectropolarimetric sensitivity, while the integration time can remain significantly shorter than a minute. The first light instrument suite of DKIST includes the Visible Spectro-Polarimeter (ViSP). ViSP is a spectrograph equipped with a polarimeter. As the spectral resolution plays a significant role in the observed RMS of the scattering polarization amplitude of Sr I (del Pino Alemán et al. 2018), ViSP has the potential to find spatial variations of the scattering polarization directly, i.e., without additional statistical averaging. Therefore, we plan to hand in a proposal as soon as possible. We estimated the expected linear polarization signal in Sr I based on the simulation by del Pino Alemán et al. (2018). Degrading the simulation to ViSP resolution results in the linear polarization shown in Figure 6.1. We will focus on tracking the temporal evolution of scattering polarization measurements and compare it to the evolution of the local radiation field, determined by the granulation. Deviations of the scattering polarization amplitude from the expected evolution given by the granulation may hint at a small-scale turbulent magnetic field. The deviation can be studied. However, it will be challenging to find optimal observational parameters to directly observe the spatial variation of the scattering polarization. To obtain the best spatio-temporal resolution, a solar region as small as a granule needs to be scanned. A more suitable instrument for this type of observations would be a filtergraph. Unfortunately, the VTF instrument (Visible Tunable Filter) of DKIST is not functional below $520 \mathrm{~nm}$.

- Concurrent center-to-limb observations with spectrograph and filtergraph type instruments. Preferably, the observations are made at the same telescope with similar conditions. These observations are necessary to study the poorly understood discrepancy between spectrograph and filtergraph observations in Sr I.

- Does the scattering polarization in Sr I at disk center depend on the solar cycle? The investigation of the center-to-limb variation of $\mathrm{Sr}$ I observed at different stages in the solar cycle so far neglected this hypothesis. Also Buehler et al. (2013) found no sign of a change in quiet Sun magnetic flux over the solar cycle. The absence 
of a cycle-dependent variation in the small-scale turbulent magnetic field could be evidence for a local origin of this field. However, whether the small-scale spatial variation of the scattering polarization in $\mathrm{Sr} I$ is independent of the solar cycle has to be investigated. A planned dedicated synoptic program at DKIST will address this question. Rempel (2020) proposed, based on 3D MHD simulations, that the quiet solar magnetism during the solar cycle may show some variation. The study of these long term variations in the spatially structured scattering polarization of Sr I complements the proposed study for shorter time scales mentioned in the first point.

- Calculate the short-term evolution of the scattering polarization in Sr I. Once spatiotemporal observations of the Sr I scattering polarization become available (see first point), spatio-temporal calculations are necessary for comparison. Potentially, this comparison constrain the generation of small-scale turbulent magnetic fields. So far, del Pino Alemán et al. (2018) investigated the scattering polarization amplitude of one snapshot in time. The same authors are currently investigating how the scattering polarization in $\mathrm{Sr}$ I changes temporally based on simulations (private communication).

- Tools and techniques, how the disk center Sr I can be interpreted in terms of the small-scale turbulent magnetic field, have to be developed. Especially the influence of depolarizing collisions at solar disk center in $\mathrm{Sr}$ I has to be taken into account carefully if the Hanle effect in Sr I is used for accurate magnetic field diagnostics. These collisional rates change with temperature (e.g., Derouich 2019). Therefore, the collisional rates may vary depending on the type of observation, i.e., the solar disk center or the solar limb. Although the combination of collision rates and small-scale turbulent magnetic field strength chosen by del Pino Alemán et al. (2018) result in scattering polarization amplitudes which are consistent with a) center-to-limb observations of Sr I and b) solar disk center observations (Zeuner et al. 2020b, in prep.), it is essential to study how sensitive the disk center scattering polarization amplitudes are with respect to the small-scale turbulent magnetic field strength and depolarization by collisions. The collisional rates used by del Pino Alemán et al. (2018) are given by Faurobert-Scholl et al. (1995).

Recently, Derouich (2019) improved the determination of depolarization rates by modeling the interaction potentials between Sr I and ionized hydrogen atoms more accurately. Further, Derouich (2019) estimated that for Sr I the magnetic field accuracy depends only weakly on the relative inaccuracy of the collision rates, i.e., a relative inaccuracy in the collision rates by a factor of two results in a error of $30 \%$ in the determined magnetic field. It is necessary to model the scattering amplitude of Sr I with the collision rates given by Derouich (2019), and compare the result with center-to-limb, as well as disk center, scattering polarization amplitudes. This will help to disentangle both depolarizing mechanisms in the solar atmosphere, magnetic fields and collisions, up to the level where magnetic fields uniquely determine the scattering polarization amplitude of $\mathrm{Sr}$ I.

- Zeuner et al. (2020) proposed a scattering model based on a single parameter, $h$. The parameter $h$ is the height of the plane above the (continuum) intensity layer where the radiation tensor component $J_{2}^{2}$ is calculated. Zeuner et al. (2020) found 
that there is an optimum value for $h$, which results in the strongest correlation of the Sr I scattering polarization with $J_{2}^{2}$. Although Zeuner et al. (2020) found that $h$ does not differ much from the expected formation height of Sr I, a detailed theoretical explanation is lacking on why $h$ has an optimum value and why the value is close to Sr I's formation height.

- Compare the radiation field tensor element $J_{2}^{2}$ provided by the simulation at different optical heights with the $J_{2}^{2}$ estimated from the continuum intensity, based on the scattering model by Zeuner et al. (2020). This will help to refine the scattering model by taking into account other aspects of anisotropic excitation. Theory predicts that the spectral line scattering polarization does not only have a strong correlation with the radiation field, but also with horizontal velocity fields (del Pino Alemán et al. 2018). Investigations if it is possible to include this contribution in the scattering model will hopefully deepen our understanding about polarization formation in $\mathrm{Sr}$.

- Improving the model for Sr I to account for spectral splitting due to the Zeeman effect in order to interpret observations accurately. In magnetically more active solar regions at disk center, Hanle and Zeeman effect coexist. For the linear polarization, which is due to scattering and the Zeeman effect, it will become challenging to disentangle both effects. It is interesting to study solar regions at disk center with slightly higher magnetic activity. On the one hand, some Hanle rotation is observed, while on the other hand also the transversal Zeeman effect is present. Observational and theoretical investigations of solar regions with simultaneously observed Hanle and Zeeman effects are needed to disentangle both. This would be one step closer to inverting Sr I data.

- Extent the method of Zeuner et al. (2020) to other scattering spectral lines in the photosphere, such as Ba II at $4554 \AA$, or, in combination with large-aperture telescopes, even weaker lines. This can help in constraining the small-scale turbulent magnetic field in the photosphere more robustly. In principle, this method could also be applied to determine the spatial structure of continuum polarization (Calvo 2019). Observations of the spatially structured continuum polarization may help to answer the question regarding the enhancement of the continuum polarization by magnetic fields in the intergranular lanes, or if the radiation anisotropy alone is responsible for it.

The results of this $\mathrm{PhD}$ project together with the outlook on the future work highlight the importance of continuing the study of spatio-temporally resolved scattering polarization of spectral lines, such as Sr I. It is anticipated that the next generation solar observatories can potentially resolve spatially structured scattering polarization directly. Nevertheless, interpretations in terms of small-scale turbulent magnetic fields most certainly will remain challenging. In this context, it is paramount to further develop the models of scattering polarization, so that the next generation of observations will enhance the understanding of the polarizing mechanisms in the solar photosphere. 

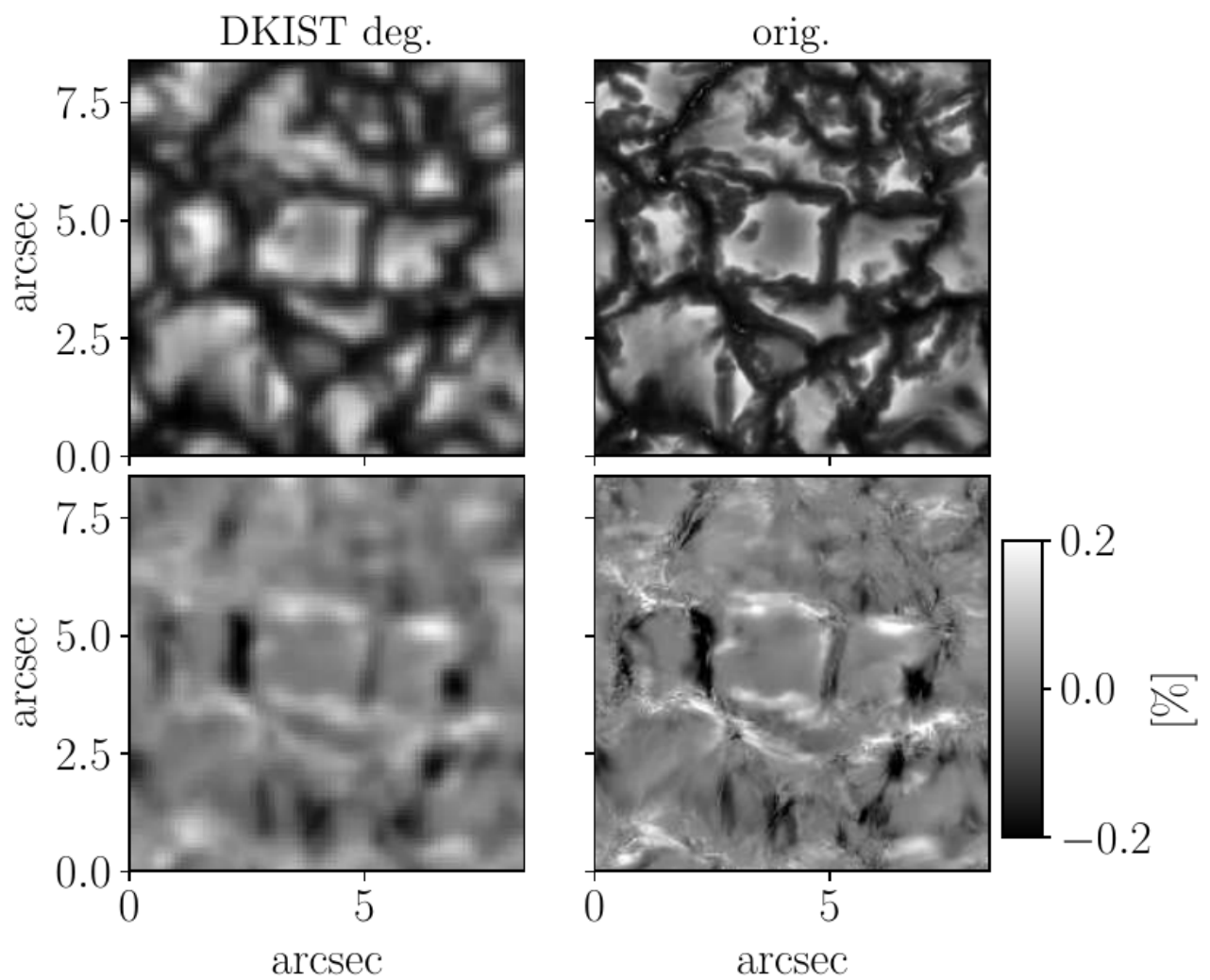

Figure 6.1: Estimated linear polarization signal of Sr I at $4607 \AA$ for a DKIST/ViSP observation (left) based on the simulation by del Pino Alemán et al. (2018) (right). The intensity image is displayed on the top. The spatial degradation is $0.1^{\prime \prime}$ pixel $^{-1}$ given by the slit width. The spectral FWHM is $32 \mathrm{m \AA}$. With this instrumental configuration, the linear polarization signal RMS is $0.05 \%$. For a minimum SNR of about two, the noise level has to be better than $3 \cdot 10^{-4} I$. Thus, an integration time of $5 \mathrm{~s}$ is required to achieve this SNR for the core of the Sr I line. 
Appendix 



\section{A Geometrical tensor}

Table A.1: Spherical tensor components $\mathcal{T}_{q}^{k}(i, \Omega)$ as given in Table 5.6 in Landi Degl' Innocenti \& Landolfi (2004), with angle definitions given in Figure A.1. The index $i$ stands for the Stokes component $I_{i}$.

$$
\begin{array}{ll}
\mathcal{T}_{0}^{0}(0, \Omega) & =1 \\
\mathcal{T}_{0,1}^{1}(0, \Omega) & =0 \\
\mathcal{T}_{0}^{2}(0, \Omega) & =\frac{1}{2 \sqrt{2}}\left(3 \cos ^{2}(\theta)-1\right) \\
\mathcal{T}_{1}^{2}(0, \Omega) & =-\frac{\sqrt{3}}{2} \sin (\theta) \cos (\theta) \mathrm{e}^{\mathrm{i} \varphi} \\
\mathcal{T}_{2}^{2}(0, \Omega) & =\frac{\sqrt{3}}{4} \sin ^{2}(\theta) \mathrm{e}^{2 \mathrm{i} \varphi}
\end{array}
$$

$$
\begin{array}{ll}
\mathcal{T}_{0}^{0}(1, \Omega) & =0 \\
\mathcal{T}_{0,1}^{1}(1, \Omega) & =0 \\
\mathcal{T}_{0}^{2}(1, \Omega) & =-\frac{\sqrt{3}}{2 \sqrt{2}} \cos (2 \gamma) \sin ^{2}(\theta) \\
\mathcal{T}_{1}^{2}(1, \Omega) & =-\frac{\sqrt{3}}{2}(\cos (2 \gamma) \cos (\theta)+\mathrm{i} \sin (2 \gamma)) \sin (\theta) \mathrm{e}^{\mathrm{i} \varphi} \\
\mathcal{T}_{2}^{2}(1, \Omega) & =\frac{\sqrt{3}}{4}\left(\cos (2 \gamma)\left(1+\cos ^{2}(\theta)\right)+2 \mathrm{i} \sin (2 \gamma) \cos (\theta)\right) \mathrm{e}^{2 \mathrm{i} \varphi}
\end{array}
$$

$$
\begin{array}{ll}
\mathcal{T}_{0}^{0}(2, \Omega) & =0 \\
\mathcal{T}_{0,1}^{1}(2, \Omega) & =0 \\
\mathcal{T}_{0}^{2}(2, \Omega) & =\frac{\sqrt{3}}{2 \sqrt{2}} \sin (2 \gamma) \sin ^{2}(\theta) \\
\mathcal{T}_{1}^{2}(2, \Omega) & =\frac{\sqrt{3}}{2}(\sin (2 \gamma) \cos (\theta)+\mathrm{i} \cos (2 \gamma)) \sin (\theta) \mathrm{e}^{\mathrm{i} \varphi} \\
\mathcal{T}_{2}^{2}(2, \Omega) & =\frac{\sqrt{3}}{4}\left(\sin (2 \gamma)\left(1+\cos ^{2}(\theta)\right)-2 \mathrm{i} \cos (2 \gamma) \cos (\theta)\right) \mathrm{e}^{2 \mathrm{i} \varphi}
\end{array}
$$

$$
\begin{array}{ll}
\mathcal{T}_{0}^{0}(3, \Omega) & =0 \\
\mathcal{T}_{0}^{1}(3, \Omega) & =\sqrt{\frac{3}{2}} \cos (\theta) \\
\mathcal{T}_{1}^{1}(3, \Omega) & =-\sqrt{\frac{3}{2}} \sin (\theta) \mathrm{e}^{2 \mathrm{i} \varphi} \\
\mathcal{T}_{0,1,2}^{2}(3, \Omega) & =0
\end{array}
$$




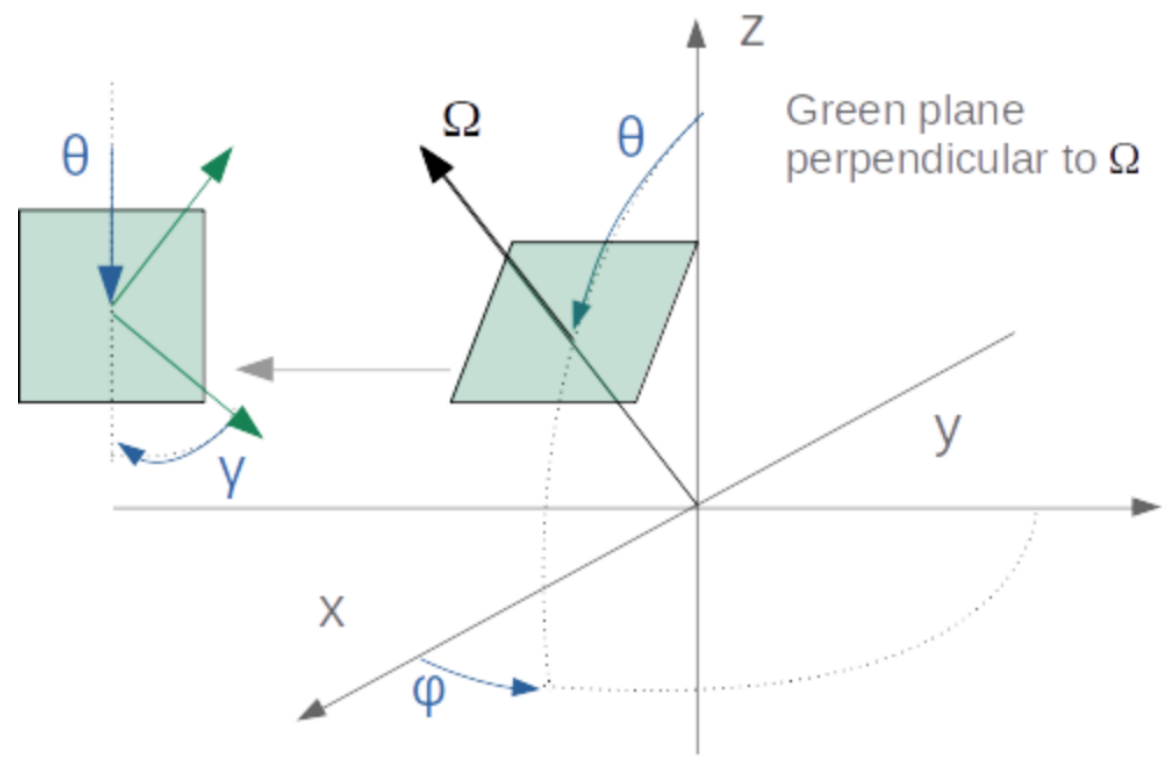

Figure A.1: Coordinate system for the geometrical tensor definition. The green coordinate system is the Stokes measurement reference system. 


\section{B Line profile}

The absorption profile $\phi$ of a two-level transition is characterized by the energy difference of the two levels, which defines the central frequency $v_{0}=\frac{E_{u}-E_{l}}{h}$, and the line broadening $\gamma$. In the atomic rest frame, the Lorentzian of the absorption and the associated dispersion profiles $\phi\left(v-v_{0}\right)$ and $\psi\left(v-v_{0}\right)$, respectively, are (Landi Degl'Innocenti \& Landolfi 2004):

$$
\begin{aligned}
& \phi\left(v-v_{0}\right)=\frac{1}{\pi} \frac{\gamma / 4 \pi}{\left(v_{0}-v\right)^{2}+(\gamma / 4 \pi)^{2}} \\
& \psi\left(v-v_{0}\right)=\frac{1}{\pi} \frac{\left(v_{0}-v\right)}{\left(v_{0}-v\right)^{2}+(\gamma / 4 \pi)^{2}} .
\end{aligned}
$$

The line broadening accounts for the finite width of the upper level, while the lower level is assumed to be infinitely sharp (both apply for the Sr I line). In general, $\gamma$ has two contributions:

$$
\gamma=\gamma_{r}+\gamma_{c}
$$

where $\gamma_{r}$ is the natural (radiative) broadening by spontaneous emission and $\gamma_{c}$ describes the broadening due to collisions. If the observers reference frame is considered, Doppler shifts due to random motions broaden the spectral line even more. The resulting absorption profile can be described as a Voigt function. The exact expressions can be found in, e.g. Landi Degl'Innocenti \& Landolfi (2004). For transforming the profile from the frequency parameter space $v$ to wavelengths $\lambda$, the relation $\phi(v) \mathrm{d} v=\phi(\lambda) \mathrm{d} \lambda$ is fullfilled.

In the presence of a magnetic field, the center of the profile is shifted according to equation (2.11) for each of the sub-levels $m$, and we write $\phi^{m}=\phi^{0}\left(\lambda-\lambda_{m}\right)$, analogously for $\psi^{m}$.

The general profile $\Phi_{q}^{k k^{\prime}}\left(J_{u}, J_{l}, \lambda\right)$ does not directly depend on the sub-levels. For Sr I, where $J_{u}=1$ and $J_{l}=0$, the elements of the general profile are given in Table B.1. 
Table B.1: General profile $\Phi_{q}^{k k^{\prime}}\left(J_{u}, J_{l}\right)$ as given in equations (A13.11) in Landi Degl'Innocenti \& Landolfi (2004) for a transition such as Sr I. For $-q,\left(\Phi_{q}^{k k^{\prime}}\right)^{*}=\Phi_{-q}^{k k^{\prime}}$.

$$
\begin{array}{ll}
\Phi_{0}^{00} & =\frac{1}{3}\left[\phi^{1}+\phi^{0}+\phi^{-1}\right] \\
\Phi_{0}^{01} & =-\Phi_{0}^{10}=\frac{1}{\sqrt{6}}\left[\phi^{1}-\phi^{-1}\right] \\
\Phi_{0}^{02} & =\Phi_{0}^{20}=\frac{1}{3 \sqrt{2}}\left[\phi^{1}-2 \phi^{0}+\phi^{-1}\right] \\
\Phi_{0}^{11} & =-\frac{1}{2}\left[\phi^{1}+\phi^{-1}\right] \\
\Phi_{1}^{11} & =-\Phi_{1}^{22}=-\frac{1}{4}\left[\phi^{1}+\mathrm{i} \psi^{1}+2 \phi^{0}+\phi^{-1}-\mathrm{i} \psi^{-1}\right] \\
\Phi_{0}^{12} & =-\Phi_{0}^{21}=-\frac{1}{3 \sqrt{2}}\left[\phi^{1}-\phi^{-1}\right] \\
\Phi_{1}^{12} & =-\Phi_{1}^{21}=-\frac{1}{4}\left[\phi^{1}+\mathrm{i} \psi^{1}-\mathrm{i} \psi^{0}-\phi^{-1}+\mathrm{i} \psi^{-1}\right] \\
\Phi_{0}^{22} & =\frac{1}{6}\left[\phi^{1}+4 \phi^{0}+\phi^{-1}\right] \\
\Phi_{2}^{22} & =-\frac{1}{2}\left[\phi^{1}+\mathrm{i} \psi^{1}+\phi^{-1}-\mathrm{i} \psi^{-1}\right]
\end{array}
$$




\section{Supplementary material related to Paper II}

\section{C.1 Fitting surfaces and analytic expressions}

To quantify the anti-correlation between the Stokes $Q / I$ and $U / I$ with $\tilde{J}_{2}^{2}$ and $\hat{J}_{2}^{2}$, respectively, seen in Figure 4.3, we fit a two-dimensional surface. A weighted multiple linear regression to each of the panels in Figure 4.3 is applied. The two free parameters for the surface are the coefficients $\alpha$ and $\beta$, spanning the space $\alpha \cdot \tilde{J}_{2}^{2}+\beta \cdot \hat{J}_{2}^{2}$. An offset is not considered, since the spatial mean of the polarization images is set to zero. The employed numerical method is an ordinary least-square minimization, where we made use of the LinearRegression module in the python package scikit-learn (Pedregosa et al. 2011). ${ }^{1}$ The weights are set according to the number of averaged Stokes image pixels per $J_{2}^{2}$ element. The fitted surfaces are plotted in Figure C.1 and the coefficients are listed in Table C.1.

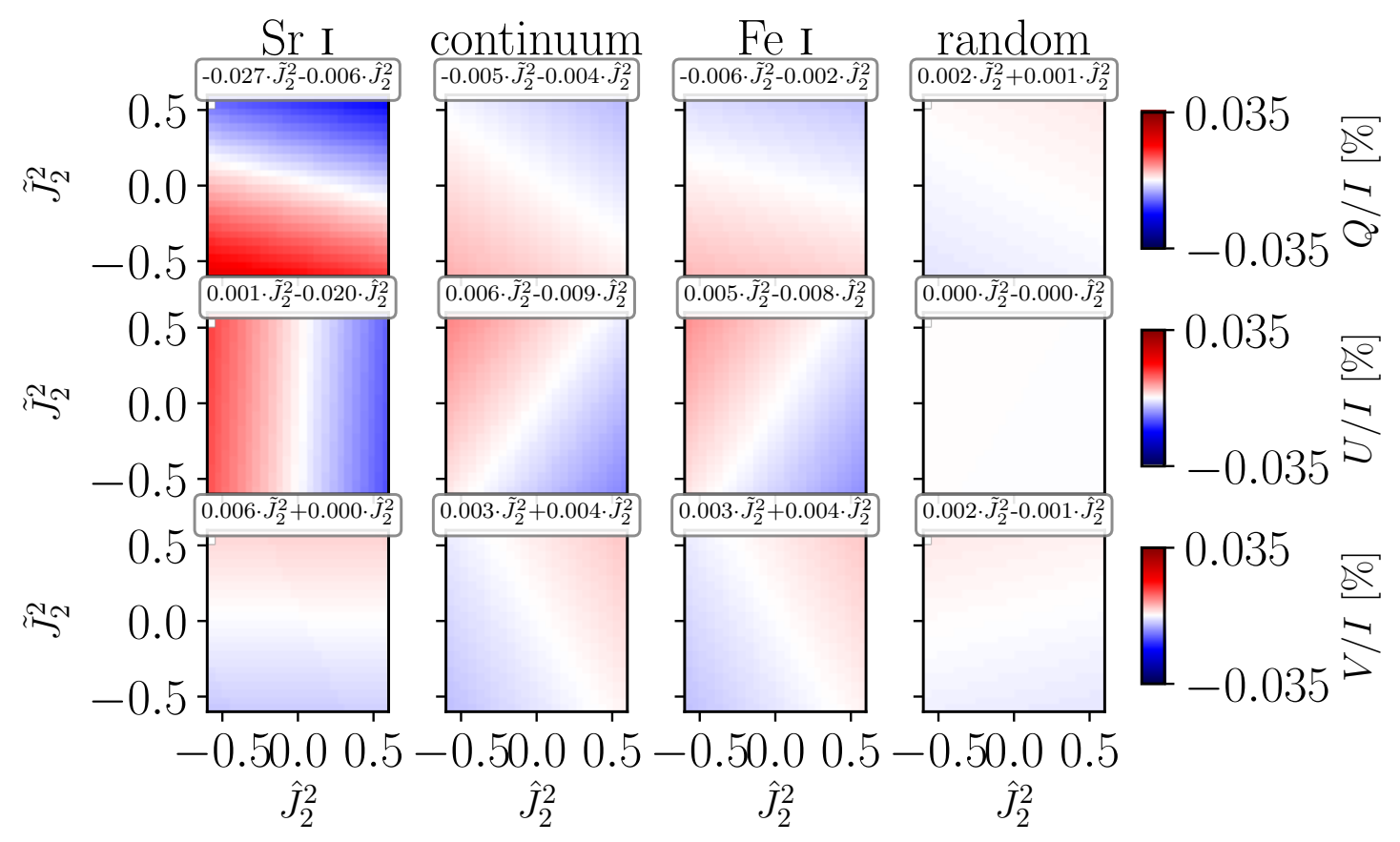

Figure C.1: Fitted two-dimensional surfaces $\alpha \cdot \tilde{J}_{2}^{2}+\beta \cdot \hat{J}_{2}^{2}$ to the maps in Figure 4.3. The coefficients $\alpha$ and $\beta$ and their corresponding $p$-values can be found in Table C.1.

1 Available here: https://scikit-learn.org/stable/. 
The coefficient is a measure of correlation. To each coefficient, we list the corresponding $p$-value, which is calculated from a two-sided $t$-test. The $p$-value indicates the probability of obtaining the same (absolute) coefficient for an uncorrelated population. The hypothesis $\left(H_{1}\right)$ is that the polarization anti-correlates with the local anisotropy, i.e. that the (absolute) coefficient is significantly larger than zero. The null-hypothesis $\left(H_{0}\right)$ opposing $H_{1}$ is, therefore, the independence of the polarization from the local anisotropy. We think, given the large sample size (number of pixels $\gg 100$ ), this is highly unlikely for $p<0.005$. The obtained coefficients and their corresponding $p$-value in Table C.1 indicate an anti-correlation in Stokes $Q / I$ and $U / I$ with $\tilde{J}_{2}^{2}$ and $\hat{J}_{2}^{2}$, respectively, in the Sri, as well as $U / I$ with $\hat{J}_{2}^{2}$ in the Fe I and continuum. However, the relevant coefficients in the continuum and $\mathrm{Fe}$ I are more than a factor two smaller than those in the $\mathrm{Sr}$. The corresponding $p$-values are a few orders of magnitude larger. This hints towards signal contamination from $\mathrm{Sr}$ I to the other observed wavelength positions due to the broad pre-filter.

We exclude residual Stokes $I$ cross-talk as a source for the anti-correlation, as the continuum and $\mathrm{Fe}$ I $\tilde{J}_{2}^{2}-\hat{J}_{2}^{2}$-diagrams should also suffer from it. The Stokes $V / I$ signals are statistically compatible with being uncorrelated with the tensor elements $\tilde{J}_{2}^{2}$ and $\hat{J}_{2}^{2}$ ( $p>0.005$, see Table C.1). The circular polarization is mostly related to the Zeeman effect, since the radiation of a quiet solar region has a very low degree of polarization. Therefore, atomic orientation and the resulting circular polarization in the scattered radiation can be neglected.

The transverse Zeeman effect as an exclusive source for either the anti-correlation pattern or the polarization amplitude can be excluded. The linear polarization in the weak field approximation (see Sect. 2.3.1) depends to second order on the Zeeman splitting, in contrast to the first order dependence of Stokes $V$. The wavelength integrated Zeeman signal amplitudes are expected to be negligible with respect to the detected linear polarization signals. They are approximately smaller by a factor three, based on the strongest $V / I$ signals in the FoV and neglecting flows. With a slightly higher Landé factor $\left(g_{e f f, F e}=1.25\right)$ and therefore higher magnetic field sensitivity compared to the $\operatorname{Sr}$ I $\left(g_{e f f, S r}=1.0\right)$, any transverse Zeeman signal in the Sr i measurement should be even more pronounced in the Fe I measurement. However, the anti-correlation found in $\mathrm{Fe}_{\mathrm{I}} U / I$ is almost a factor four smaller than in Sri $U / I$. This hints towards Sr I signals leaking into the Fe I measurement (most likely due to the broad pre-filter), in contrast to a transverse Zeeman signature. This argument is supported by the fact that an anti-correlation of the same order as in the Fe I line is visible in the continuum. Transverse Zeeman signals are additionally expected to be randomly distributed in the highly mixed-polarity quiet Sun intergranular lanes and should therefore be independent of the local radiation field anisotropy.

The only free parameter of the model to calculate the anisotropy maps $\left(\tilde{J}_{2}^{2}\right.$ and $\left.\hat{J}_{2}^{2}\right)$ is the height $h$ of the assumed scattering layer (see Figure 4.2). We tested the robustness of our method with respect to $h$ by calculating the coefficients $\alpha$ and $\beta$ for $1 \lesssim h \lesssim 5$. It is found that the highest anti-correlations (largest coefficients) exist for $h=3$ pixel. That an optimum value for this parameter exists is a consequence of the coherence length of the $J_{2}^{2}$ components. Therefore, we restricted our presented analysis to the case $h=3$ pixel.

Instead of numerically fitting the model $\alpha \cdot \tilde{J}_{2}^{2}+\beta \cdot \hat{J}_{2}^{2}$ independently to the diagrams of $Q / I$ and $U / I$ in Figure 4.3, we propose to use an analytic expression for the coefficients $\alpha$ and $\beta$. This step allows for a direct calculation of the angle $\zeta$ between the radiation field tensor element $J_{2}^{2}$ and the observed polarization plane. 
Table C.1: Coefficients $\alpha$ and $\beta$ deduced by weighted multiple linear regression to fit the surfaces $\alpha \cdot \tilde{J}_{2}^{2}+\beta \cdot \hat{J}_{2}^{2}$ to the maps in Figure 4.3. The probability $p$ from a two-sided $t$-test to observe a coefficient as large or larger than these for an uncorrelated population is added. Low $p$-values $(p<0.005)$ indicate incompatibility with the null-hypothesis of uncorrelated data.

\begin{tabular}{c|c|c|c||c|c|c||c|c|c}
\hline \hline & \multicolumn{3}{|c|}{ Sr I } & \multicolumn{3}{c|}{ continuum } & \multicolumn{3}{c}{$\mathrm{Fe}_{\mathrm{I}}$} \\
\hline & $Q / I$ & $U / I$ & $V / I$ & $Q / I$ & $U / I$ & $V / I$ & $Q / I$ & $U / I$ & $V / I$ \\
\hline$\alpha$ & -0.027 & -0.001 & 0.006 & -0.005 & 0.006 & 0.003 & -0.006 & 0.005 & 0.003 \\
$p$ & $<10^{-6}$ & 0.645 & 0.017 & 0.076 & 0.003 & 0.144 & 0.169 & 0.065 & 0.251 \\
\hline$\beta$ & -0.006 & -0.020 & 0.000 & -0.004 & -0.009 & 0.004 & -0.002 & -0.008 & 0.004 \\
$p$ & 0.075 & $<10^{-6}$ & 0.878 & 0.208 & $<10^{-4}$ & 0.011 & 0.677 & $<10^{-3}$ & 0.0225 \\
\hline
\end{tabular}

We rewrite for clarity $\mathbf{x}=\left(x_{1}, x_{2}\right)=\left(\tilde{J}_{2}^{2}, \hat{J}_{2}^{2}\right)$. We refer to the $\tilde{J}_{2}^{2}-\hat{J}_{2}^{2}$-diagram for the observed $Q / I$ and $U / I$ as $f(\mathbf{x})$ and $g(\mathbf{x})$, respectively. The weights $w(\mathbf{x})=n(\mathbf{x})$ are the number of accumulated Stokes pixels within one $\tilde{J}_{2}^{2}-\hat{J}_{2}^{2}$-diagram pixel. The model we fitted independently is $q, u=\alpha \cdot x_{1}+\beta \cdot x_{2}$. From the Figure 4.3 we are confident that $q$ and $u$ show orthogonal behaviour, therefore we adjust the fitted model to $q^{\prime}=\alpha^{\prime} \cdot x_{1}-\beta^{\prime} \cdot x_{2}$ and $u^{\prime}=\beta^{\prime} \cdot x_{1}+\alpha^{\prime} \cdot x_{2}$. Thereby, we constrain the coefficients to be orthogonal, i.e., $q^{\prime} \cdot u^{\prime}=0$. Note that there is an ambiguity, as exchanging $\beta^{\prime}$ with $-\beta^{\prime}$ is also satisfying the orthogonality condition. But this ambiguity is resolved from the definition of the angle $\zeta$ (see below). Explicitly formulating the minimization problem leads to:

$$
\begin{aligned}
& M_{1}=\sum_{\mathbf{x}} \frac{\left(f(\mathbf{x})-q^{\prime}(\mathbf{x})\right)^{2}}{w(\mathbf{x})} \\
& M_{2}=\sum_{\mathbf{x}} \frac{\left(g(\mathbf{x})-u^{\prime}(\mathbf{x})\right)^{2}}{w(\mathbf{x})}
\end{aligned}
$$

where we need to satisfy for $i=1,2$ the condition

$$
\frac{\partial M_{i}}{\partial \alpha^{\prime}}+\frac{\partial M_{i}}{\partial \beta^{\prime}}=0
$$

After some algebra, we can explicitly solve for $\alpha^{\prime}$ and $\beta^{\prime}$. The resulting expressions are

$$
\alpha^{\prime}=o-\beta^{\prime} \cdot s
$$

and

$$
\beta^{\prime}=\frac{o-d}{s-c}
$$


with

$$
\begin{aligned}
& o=\frac{\sum_{\mathbf{X}}\left(g(\mathbf{x}) \cdot\left(x_{1}+x_{2}\right)\right) / w(\mathbf{x})}{\sum_{\mathbf{x}}\left(x_{2}^{2}+x_{1} \cdot x_{2}\right) / w(\mathbf{x})} \\
& d=\frac{\sum_{\mathbf{x}}\left(f(\mathbf{x}) \cdot\left(x_{1}-x_{2}\right)\right) / w(\mathbf{x})}{\sum_{\mathbf{x}}\left(x_{1}^{2}-x_{1} \cdot x_{2}\right) / w(\mathbf{x})} \\
& c=\frac{\sum_{\mathbf{x}}\left(x_{2}^{2}-x_{2} \cdot x_{1}\right) / w(\mathbf{x})}{\sum_{\mathbf{x}}\left(x_{1}^{2}-x_{1} \cdot x_{2}\right) / w(\mathbf{x})} \\
& s=\frac{\sum_{\mathbf{x}}\left(x_{1}^{2}+x_{2} \cdot x_{1}\right) / w(\mathbf{x})}{\sum_{\mathbf{x}}\left(x_{2}^{2}+x_{1} \cdot x_{2}\right) / w(\mathbf{x})}
\end{aligned}
$$

Table C.2 shows the values for $\alpha$ and $\beta$ calculated with equation (C.3) and equation (C.4)

Table C.2: Coefficients $\alpha^{\prime}$ and $\beta^{\prime}$ calculated with equations (C.3) and (C.4) for the observation in Zeuner et al. (2020). The equations account for the orthogonality of $Q$ and $U$.

\begin{tabular}{cccc}
\hline \hline & Sr I & continuum & Fe I \\
\hline$\alpha^{\prime}$ & -0.0179 & -0.0081 & -0.0042 \\
$\beta^{\prime}$ & 0.0014 & 0.0056 & 0.0002 \\
\hline
\end{tabular}

using the data described in Zeuner et al. (2020). The values are consistent with the coefficients found in Sect. 4.5 by numerical minimization. However, $\alpha^{\prime}=-0.0179$ calculated here for the Sr I data is lower than the mean of the independently fit coefficients $0.5 \cdot\left(\alpha_{Q}+\beta_{U}\right)=0.024$ (see Figure C.1). This is expected, as the added constraint of orthogonality between $Q$ and $U$ is not perfectly fullfilled for noisy data (see the nodal (white) lines in Figure C.1).

In addition to a faster and more robust formalism to calculate the coefficients, it is possible to define now the angle $\zeta=90^{\circ}-\arctan \left(\frac{|\alpha|}{\beta}\right)$, which represents the offset angle between the inferred signal direction of $Q / I$ and $U / I$ with respect to the ideal case, where the measured $Q$ direction coincides with the image plane coordinate system (e.g. $+Q$ is parallel to the image plane $y$-axis), see also Sec. 2.3.2.3 for the definition of $\zeta$. We find $\zeta \approx$ $4.5^{\circ}$ in the data presented in Zeuner et al. (2020). To test the reliability of the calculation of $\zeta$ presented in this section, we also calculated the coefficients $\alpha$ and $\beta$ for data which we rotated by an angle of $-4^{\circ}$ in order to maximize the alignment between the $Q=0$ direction given by the $J_{2}^{2}$ map calculation based on the image coordinate system and the actually measured $Q$ direction data and find an angle of $\zeta_{\text {rot }=4} \approx 0.8^{\circ}$. The origins of this angle can be manifold and, furthermore, the reasons are indistinguishable within the limits of our data set. Thus the issue will only be resolved in future observations. One reason can be identified in the observational process. As the polarimeter was only temporally installed at the telescope, no extra effort was made to align the direction of measured $Q$ with the observed image plane to great accuracy. The issue of noisy data also leaves an additional uncertainty of about $5^{\circ}$, see Zeuner et al. (2020) for details. 


\section{C.2 Time evolution of the radiation field coherence}

At solar disk center, the coherences of the radiation field tensor as sources of linear polarization are very important. These coherences are a consequence of the lack of axial symmetry. Therefore, they are localized in the regions with less symmetry. The most extreme case of the lowest symmetry are the boundaries between the granules and the intergranular lanes. These are the regions where also the $\left|J_{2}^{2}\right|$ are highest. As an example, we plot the intensity instead of the polarization as a function of $J_{2}^{2}$ in Figure C.2. Clearly, the center for all three wavelength positions is brighter than the rest. The darker regions are located at more extreme values of $\left|J_{2}^{2}\right|$. Because of the broad pre-filter (Zeuner et al. 2020), even the Sr I and Fe I line intensity data is dominated by the continuum. Therefore, the darker regions correspond to intergranules. The boundary between granules and intergranules are also those regions were the velocity changes from upwards to downwards, at the height relevant for the Sr I spectral line. Consequently, it is to be expected that the time evolution of the granulation is relevant for the location of these boundaries. The regions were the radiation field tensor coherences are bigger also the temporal changes are faster. Therefore, where the linear polarization signals are localized, the temporal evolution introduces an extra smearing effect.
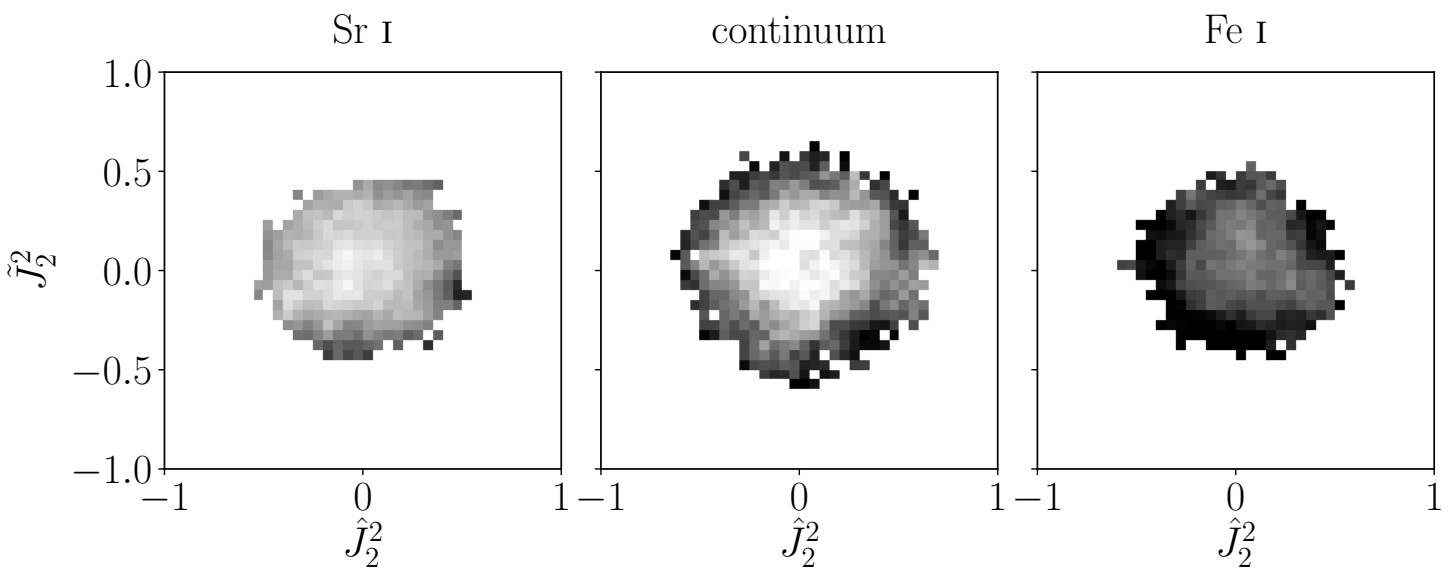

Figure C.2: Stokes $I$ as a function of $J_{2}^{2}$, similar to Figure 4.3 for the polarimetric Stokes parameters. As expected, bright regions (the center of granules) are located at low $\left|J_{2}^{2}\right|$ values, while low symmetry regions such as the boundary of granules and intergranules are found at high $\left|J_{2}^{2}\right|$ values. The intensity is normalized to one, the plotted range for the continuum is $0.79-0.84$, while for the other two the plotted range is $0.75-0.80$. The Fe I line is deeper than the Sr I line, therefore it is darker.

In order to study the temporal degradation of the linear polarization signals in the Sr I line, we use successive temporally averaged Stokes images. The diagrams in Figure 4.3 should be affected by the temporal integration step before the classification, as solar evolution is spatially smearing the intensity images. We calculate the $\tilde{J}_{2}^{2}-\hat{J}_{2}^{2}$-diagrams for the Sr I data and fitted the coefficients $\alpha$ and $\beta$ for each temporal integration step (see previous section). Figure C. 4 shows both coefficients for all three Stokes components with a fast decay of the coefficient's value within the first $10 \mathrm{~s}$. We fitted a power law and find 
an exponent close to two. This suggests that the decay is caused by the averaging, which reduces mainly the extreme values in the $J_{2}^{2}$ maps. As a consequence, long integration times during observations lead to a reduced scattering signal. For timescales of minutes, which is the typical solar evolution timescale, the scattering polarization should still be measurable.

del Pino Alemán et al. (2018) already speculated that observing with high temporal resolution is crucial to detect the polarization signals within solar evolution timescales. However, a detailed theoretical investigation of the dynamics of the polarization signals and the connection to the temporally evolving magnetic field is still missing.

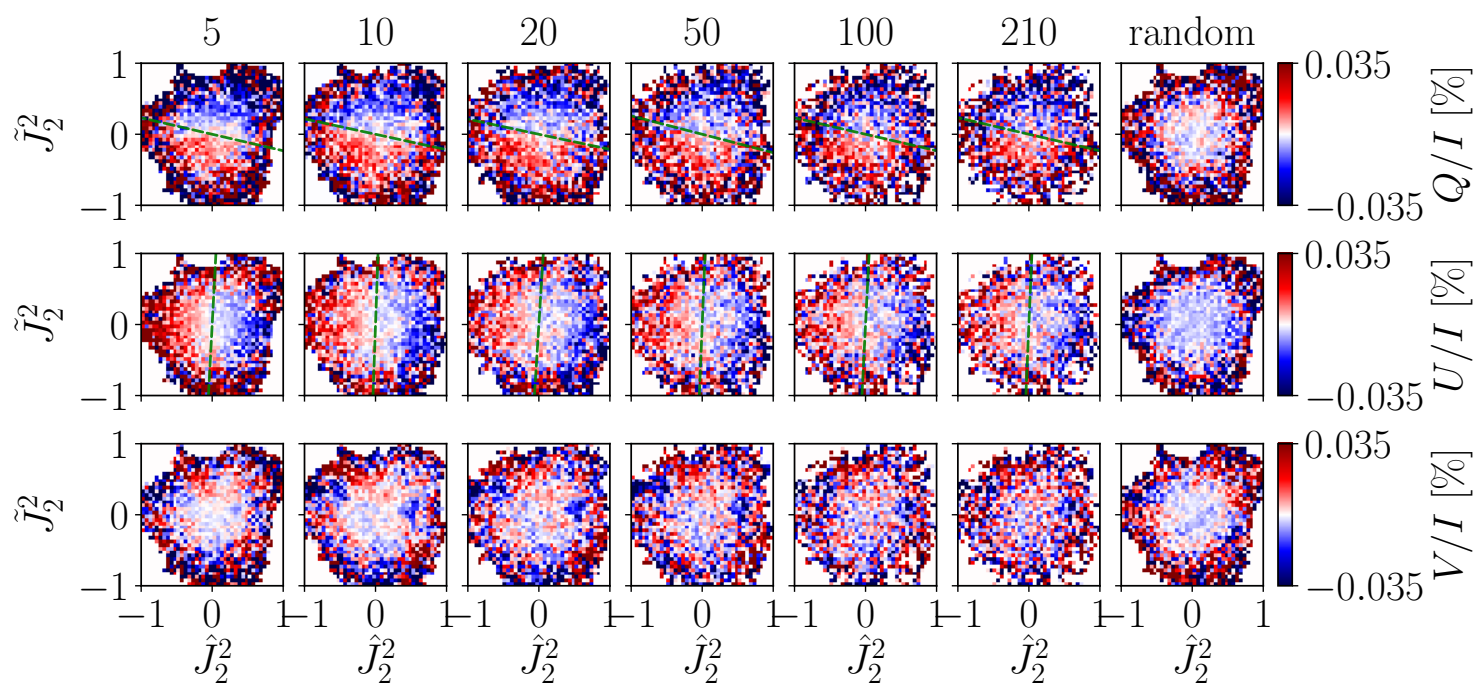

Figure C.3: Temporal degradation of the Sr I polarization signals. The Stokes images are temporally averaged for different integration time steps $t$ (in seconds, on top of each column) before calculating the $J_{2}^{2}$ maps. After classification, the two-dimensional histograms of the polarization signals with $\tilde{J}_{2}^{2}-\hat{J}_{2}^{2}$-bins are averaged, in the same way as explained by Zeuner et al. (2020). We fitted two-dimensional surfaces $\left(L=\alpha \cdot \tilde{J}_{2}^{2}+\beta \cdot \hat{J}_{2}^{2}\right)$ as explained in Zeuner et al. (2020). The green dashed lines represent $L=0$. 


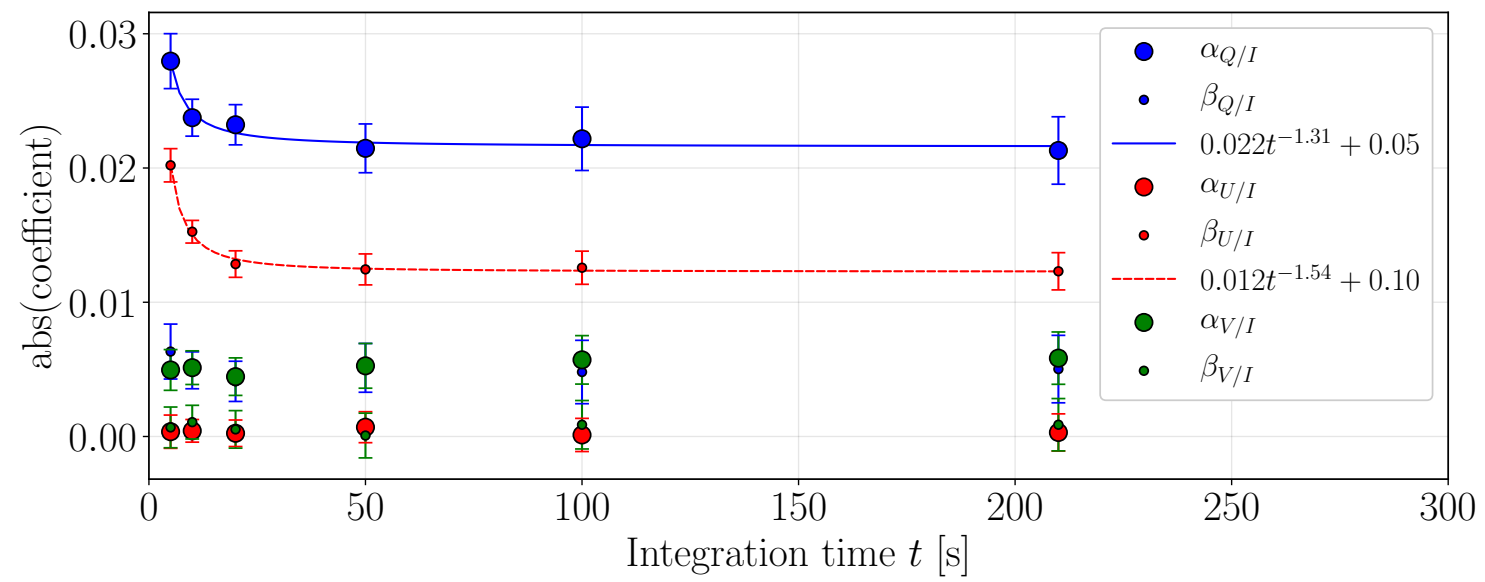

Figure C.4: Absolute value of the coefficients $\alpha$ and $\beta$ as a function of the integration time step $t$ obtained in Figure C.3. A power law is fitted to the significant coefficients $\alpha_{Q / I}$ and $\beta_{U / I}$. The error bars are taken from the uncertainty of the two-dimensional fit. 



\section{Bibliography}

Alsina Ballester, E., Belluzzi, L., \& Trujillo Bueno, J. 2017, ApJ, 836, 6

Archontis, V. \& Vlahos, L. 2019, Phil. Trans. R. Soc. A, 377

Beck, C., Bellot Rubio, L. R., Kentischer, T. J., Tritschler, A., \& del Toro Iniesta, J. C. 2010, A\&A, 115, 10

Bellot Rubio, L. \& Orozco Suárez, D. 2019, Living Rev. Sol. Phys., 123

Belluzzi, L. \& Trujillo Bueno, J. 2012, ApJL, 750, L11

Bevington, P. R. \& Robinson, D. K. 2003, Data reduction and error analysis for the physical sciences, 3rd edn. (McGraw-Hill)

Bianda, M., Berdyugina, S., Gisler, D., et al. 2018, A\&A, 614, A89

Bianda, M., Ramelli, R., Anusha, L. S., et al. 2011, A\&A, 530, L13

Bianda, M., Solanki, S. K., \& Stenflo, J. O. 1998, A\&A, 331, 760

Blum, K. 2012, Density Matrix Theory and Applications, 3rd edn. (Springer)

Bommier, V., Derouich, M., Landi Degl'Innocenti, E., Molodij, G., \& Sahal-Bréchot, S. 2005, A\&A, 432, 295

Born, M. \& Wolf, E. 1970, Principles of Optics, 4th edn. (Pergamon Press), 859

Borrero, J. M., Jafarzadeh, S., Schüssler, M., \& Solanki, S. K. 2015, Space Sci. Rev., 210, 275

Borrero, J. M., Jafarzadeh, S., Schüssler, M., \& Solanki, S. K. 2017, Space Sci. Rev., 210, 275

Brandenburg, A. \& Subramanian, K. 2005, Phys. Rep., 417, 1

Buehler, D., Lagg, A., \& Solanki, S. K. 2013, A\&A, 555, A33

Calvo, F. 2019, PhD thesis, Université de Genève

Casini, R., Landi Degl'Innocenti, E., \& Iwamae, A. 2008, Astrophysical Plasmas (Berlin, Heidelberg: Springer Berlin Heidelberg), 247-287

Cattaneo, F. 1999, ApJL, 515, L39 
Chandrasekhar, S. 1960, Radiative Transfer (New York: Dover Publications)

Christensen-Dalsgaard, J. 2020, arXiv:2007.06488

Collados, M. 1999, in Astronomical Society of the Pacific Conference Series, Vol. 184, Third Advances in Solar Physics Euroconference: Magnetic Fields and Oscillations, ed. B. Schmieder, A. Hofmann, \& J. Staude, 3-22

Danilovic, S., Beeck, B., Pietarila, A., et al. 2010a, ApJL, 723, L149

Danilovic, S., Schüssler, M., \& Solanki, S. K. 2010b, A\&A, 513, A1

Danilovic, S., van Noort, M., \& Rempel, M. 2016, A\&A, 593, A93

de Wijn, A. G., Stenflo, J. O., Solanki, S. K., \& Tsuneta, S. 2009, Space Sci. Rev., 144, 275

del Pino Alemán, T., Trujillo Bueno, J., Stěpán, J., \& Shchukina, N. 2018, ApJ, 863, 20

del Toro Iniesta, J. C. 2004, Introduction to Spectropolarimetry (Cambridge University Press)

del Toro Iniesta, J. C. \& Collados, M. 2000, Appl. Opt., 39, 1637

Derks, A., Beck, C., \& Martínez Pillet, V. 2018, A\&A, 615, A22

Derouich, M. 2019, ApJ, 887, 260

Dhara, S. K., Capozzi, E., Gisler, D., et al. 2019, A\&A, 630, A67

Domínguez Cerdeña, I., Sánchez Almeida, J., \& Kneer, F. 2006, ApJ, 636, 496

Donati, J. F., Semel, M., Carter, B. D., Rees, D. E., \& Collier Cameron, A. 1997, MNRAS, 291,658

Donati, J. F., Semel, M., Rees, D. E., Taylor, K., \& Robinson, R. D. 1990, A\&A, 232, L1

Faurobert, M., Arnaud, J., Vigneau, J., \& Frisch, H. 2001, A\&A, 378, 627

Faurobert, M., Frisch, H., \& Skumanich, A. 1987, Publications of the Astronomical Institute of the Czechoslovak Academy of Sciences, 1, 205

Faurobert-Scholl, M. 1993, A\&A, 268, 765

Faurobert-Scholl, M., Feautrier, N., Machefert, F., Petrovay, K., \& Spielfiedel, A. 1995, A\&A, 298, 289

Fluri, D. M. \& Stenflo, J. O. 1999, A\&A, 341, 902

Fox, N. J., Velli, M. C., Bale, S. D., et al. 2016, Space Sci. Rev., 204, 7

Frisch, H. 1996, Sol. Phys., 164, 49 
Frutiger, C., Solanki, S. K., Fligge, M., \& Bruls, J. H. 2000, A\&A, 358, 1109

Gandorfer, A. 2000, The Second Solar Spectrum: A high spectral resolution polarimetric survey of scattering polarization at the solar limb in graphical representation. Volume I: $4625 \AA$ to $6995 \AA$ (VdF, Zurich)

Gandorfer, A. 2002, The Second Solar Spectrum: A high spectral resolution polarimetric survey of scattering polarization at the solar limb in graphical representation. Volume II: $3910 \AA$ to $4630 \AA$ (Zurich: VdF)

Gandorfer, A. 2005, The Second Solar Spectrum: A high spectral resolution polarimetric survey of scattering polarization at the solar limb in graphical representation. Volume III: $3160 \AA$ to $3915 \AA$ (VdF, Zurich)

Graham, J. P., Danilovic, S., \& Schüssler, M. 2009, ApJ, 693, 1728

Haigh, J. D., Lockwood, M., \& Giampapa, M. S. 2004, The Sun, Solar Analogs and the Climate (Springer)

Hale, G. E., Ellerman, F., Nicholson, S. B., \& Joy, A. H. 1919, ApJ, 49, 153

Hanle, W. 1924, Zeitschrift für Physik, 30, 93

Hapgood, M. 2019, Space Weather, 17, 950

Harvey, J., Brault, J., Stenflo, J., \& Zwaan, C. 1980, in BAAS, Vol. 12, 476

Hathaway, D. H. 2015, Living Rev. Sol. Phys., 12, 4

Hirzberger, J., Bonet, J. A., Vázquez, M., \& Hanslmeier, A. 1999, ApJ, 515, 441

Howard, R. \& Stenflo, J. O. 1972, Sol. Phys., 22, 402

Hubeny, I. \& Mihalas, D. 2015, Theory of Stellar Atmospheres (Princeton and Oxford: Princeton University Press)

Iglesias, F. A. 2016, PhD thesis, University of Braunschweig

Iglesias, F. A. \& Feller, A. 2019, Opt. Eng., 58, 082417

Iglesias, F. A., Feller, A., \& Nagaraju, K. 2015, Appl. Opt., 54, 5970

Iglesias, F. A., Feller, A., Nagaraju, K., \& Solanki, S. K. 2016, A\&A, 590, A89

Jackson, J. D. 1998, Classical Electrodynamics, 3rd Edition (Wiley)

Jacobs, V. L. 1998, Proceedings of the Japan-U.S. Workshop and International Seminar on Plasma Polarization Spectroscopy, 187

Keller, C. U. 1996, Sol. Phys., 164, 245

Keller, C. U., Deubner, F. L., Egger, U., Fleck, B., \& Povel, H. P. 1994a, A\&A, 286, 626 
Keller, C. U., Povel, H.-P., \& Stenflo, J. O. 1994b, in Society of Photo-Optical Instrumentation Engineers (SPIE) Conference Series, Vol. 2265, Proc. SPIE, ed. D. H. Goldstein \& D. B. Chenault, 222-230

Kentischer, T. J., Schmidt, W., Sigwarth, M., \& Uexküll, M. v. 1998, A\&A, 340, 569

Khomenko, E. 2006, ASP Conference Series, 354, 63

Khomenko, E., Vitas, N., Collados, M., \& de Vicente, A. 2017, A\&A, 604, A66

Kleint, L., Berdyugina, S. V., Shapiro, A. I., \& Bianda, M. 2010, A\&A, 524, A37

Kleint, L., Shapiro, A. I., Berdyugina, S. V., \& Bianda, M. 2011, A\&A, 536, A47

Krishnappa, N. \& Feller, A. 2012, Appl. Opt., 51, 7953

Lagg, A., Solanki, S. K., Collados Vera, M., et al. 2016, A\&A, A6

Landau, L. \& Lifshitz, E. 2013, Mechanics and Electrodynamics (Elsevier Science)

Landé, A. 1923, Zeitschrift fur Physik, 15, 189

Landi Degl'Innocenti, E. \& Landolfi, M. 2004, Polarization in spectral lines (Kluwer Academic Publishers, Dordrecht)

Lites, B. W. 1987, Appl. Opt., 26, 3838

Lites, B. W. 2011, ApJ, 737, 52

Lites, B. W., Centeno, R., \& McIntosh, S. W. 2014, PASJ, 66, S4

Lites, B. W., Kubo, M., Socas-Navarro, H., et al. 2008, ApJ, 672, 1237

Lites, B. W., Leka, K. D., Skumanich, A., Martínez Pillet, V., \& Shimizu, T. 1996, ApJ, 460,1019

Livingston, W. C. \& Harvey, J. 1975, Bulletin of the American Astronomical Society, 7, 346

Löfdahl, M. G. 2002, in Society of Photo-Optical Instrumentation Engineers (SPIE) Conference Series, Vol. 4792, Proc. SPIE, ed. P. J. Bones, M. A. Fiddy, \& R. P. Millane, $146-155$

Malherbe, J.-M., Moity, J., Arnaud, J., \& Roudier, T. 2007, A\&A, 462, 753

Manso Sainz, R., Landi Degl'Innocenti, E., \& Trujillo Bueno, J. 2004, ApJ, 614, L89

Manso Sainz, R., Roncero, O., Sanz-Sanz, C., et al. 2014, ApJ, 788, 118

Manso Sainz, R. \& Trujillo Bueno, J. 2011, ApJ, 743, 12

Maunder, E. W. 1904, MNRAS, 64, 747 
Mihalas, D. 1978, Stellar atmospheres (San Francisco: W.H. Freeman)

Milkey, R. W. \& Mihalas, D. 1973, ApJ, 185, 709

Mitchell, A. C. G. \& Zemansky, M. W. 2009, Resonance Radiation and Excited Atoms (Cambridge Univ., Cambridge)

Moll, R., Pietarila Graham, J., Pratt, J., et al. 2011, ApJ, 736, 36

Moruzzi, G. \& Strumia, F., eds. 1991, The Hanle Effect and Level-Crossing Spectroscopy (Springer Science+Business Media LLC)

Neckel, H. 1999, Sol. Phys., 184, 421

Oklopcic, A., Hirata, C., Montero Camacho, P., \& Silva, M. 2019, in AAS/Division for Extreme Solar Systems Abstracts, Vol. 51, AAS/Division for Extreme Solar Systems Abstracts, 103.05

Pedregosa, F., Varoquaux, G., Gramfort, A., et al. 2011, J. Mach. Learn. Res., 12, 2825

Petrovay, K. 2001, Space Sci. Rev., 95, 9

Petrovay, K. \& Szakály, G. 1993, A\&A, 274, 543

Povel, H., Aebersold, H., \& Stenflo, J. O. 1990, Appl. Opt., 29, 1186

Qutub, S., Derouich, M., \& Zaheer Ahmad, B. 2020, arXiv e-prints, arXiv:2010.06982

Ramelli, R., Balemi, S., Bianda, M., et al. 2010, in Ground-based and Airborne Instrumentation for Astronomy III, ed. I. S. McLean, S. K. Ramsay, \& H. Takami, Vol. 7735, International Society for Optics and Photonics (SPIE), 778 - 789

Reiners, A. 2012, Living Rev. Sol. Phys., 9, 1

Rempel, M. 2014, ApJ, 789, 132

Rempel, M. 2020, ApJ, 894, 140

Riley, P., Baker, D., Liu, Y. D., et al. 2017, Space Sci. Rev., 214, 21

Sampoorna, M., Trujillo Bueno, J., \& Landi Degl'Innocenti, E. 2010, ApJ, 722, 1269

Sánchez Almeida, J. 2005, A\&A, 438, 727

Schekochihin, A. A., Cowley, S. C., Taylor, S. F., Maron, J. L., \& McWilliams, J. C. 2004, ApJ, 612, 276

Schou, J., Borrero, J. M., Norton, A. A., et al. 2012, Sol. Phys., 275, 327

Schüssler, M. \& Vögler, A. 2008, A\&A, 481, 5

Semel, M., Donati, J. F., \& Rees, D. E. 1993, A\&A, 278, 231 
Shchukina, N. G. \& Trujillo Bueno, J. 2013, in IAU Symposium, Vol. 294, Solar and Astrophysical Dynamos and Magnetic Activity, ed. A. G. Kosovichev, E. de Gouveia Dal Pino, \& Y. Yan, 107-118

Skumanich, A., Lites, B. W., Martínez Pillet, V., \& Seagraves, P. 1997, Astrophys. J. Suppl. S., 110,357

Snik, F., de Wijn, A. G., Ichimoto, K., et al. 2010, A\&A, 519, A18

Socas-Navarro, H., Elmore, D., Asensio Ramos, A., \& Harrington, D. M. 2011, A\&A, 531, A2

Solanki, S. K. 1987, PhD thesis, ETH Zürich

Solanki, S. K. 2009, in Astronomical Society of the Pacific Conference Series, Vol. 405, Solar Polarization 5: In Honor of Jan Stenflo, ed. S. V. Berdyugina, K. N. Nagendra, \& R. Ramelli, 135

Solanki, S. K., Inhester, B., \& Schüssler, M. 2006, Rep. Prog. Phys, 69, 563

Steiner, O. \& Rezaei, R. 2012, ASP Conference Series, 456, 3

Stenflo, J. 1994, Solar Magnetic Fields: Polarized Radiation Diagnostics (SpringerScience+Business Media, B.V.)

Stenflo, J. O. 1973, Sol. Phys., 32, 41

Stenflo, J. O. 1982, Sol. Phys., 80, 209

Stenflo, J. O. 1988, Sol. Phys., 114, 1

Stenflo, J. O. 2011, A\&A, 529, A42

Stenflo, J. O. 2012a, A\&A, 547, A93

Stenflo, J. O. 2012b, A\&A, 541, A17

Stenflo, J. O. 2013, A\&A Rev., 21, 66

Stenflo, J. O. 2014, FTS Atlas 2: http://data.irsol.ch/data_archive/\#ftsv

Stenflo, J. O., Bianda, M., Keller, C. U., \& Solanki, S. K. 1997, A\&A, 322, 985

Stenflo, J. O. \& Keller, C. U. 1996, Nature, 382, 588

Stenflo, J. O. \& Keller, C. U. 1997, A\&A, 934, 927

Stenflo, J. O., Keller, C. U., \& Gandorfer, A. 1998, A\&A, 329, 319

Stenflo, J. O., Twerenbold, D., Harvey, J. W., \& Brault, J. W. 1983, A\&A Suppl. Ser., 54, 505

Štěpán, J., Bestard, J. J., \& Trujillo Bueno, J. 2020, A\&A, 636, A24 
Stix, M. 2004, The Sun, 2nd edn. (Springer)

Stokes, G. G. 1852, Philosophical Transactions of the Royal Society of London Series I, 142,463

Tritschler, A., Schmidt, W., Langhans, K., \& Kentischer, T. 2002, Sol. Phys., 211, 17

Trujillo Bueno, J. 2001, in ASP Conference Series, Vol. 236, 161-195

Trujillo Bueno, J. 2003, Symposium - International Astronomical Union, 210, 243-258

Trujillo Bueno, J. 2003a, in ASP Conference Series, Vol. 307, 407-424

Trujillo Bueno, J. 2003b, in ASP Conference Series, Vol. 288, 551-582

Trujillo Bueno, J. 2005, Proceedings of the 11th European Solar Physics Meeting

Trujillo Bueno, J., Landi Degl'Innocenti, E., Casini, R., \& Martínez Pillet, V. 2005, in Proceedings of the International Scientific Conference on Chromospheric and Coronal Magnetic fields (ESA SP-596, November 2005), ed. Innes, D. E., Lagg, A., \& Solanki, S. K., Katlenburg-Lindau

Trujillo Bueno, J. \& Manso Sainz, R. 1999, ApJ, 516, 436

Trujillo Bueno, J. \& Shchukina, N. 2007, ApJL, 664, L135

Trujillo Bueno, J., Shchukina, N., \& Asensio Ramos, A. 2004, Nature, 430, 326

Unno, W. 1959, ApJ, 129, 375

van Noort, M., Rouppe van der Voort, L., \& Löfdahl, M. G. 2005, Sol. Phys., 228, 191

van Regemorter, H. 1962, ApJ, 136, 906

Vögler, A. \& Schüssler, M. 2007, A\&A, 465, L43

Vögler, A., Shelyag, S., Schüssler, M., et al. 2005, Astronomy \& Astrophysics, 429, 335

Zeeman, P. 1897, ApJ, 5, 332

Zeuner, F., Feller, A., Iglesias, F. A., \& Solanki, S. K. 2018, A\&A, 619, A179

Zeuner, F., Manso Sainz, R., Feller, A., et al. 2020, ApJL, 893, L44 



\section{Acknowledgements}

Starting a doctoral thesis means entering unfamiliar terrain - for someone without a formal training in astrophysics and starting a PhD in solar physics this is literally true. I was lucky to have experienced and inspiring guides, who helped me find my way and who helped me overcoming obstacles.

I would particularly like to thank my doctoral adviser Prof. Sami K. Solanki. This thesis greatly benefited from his scientific support, wealth of knowledge and patience with my writing. I sincerely thank my thesis advisors Alex Feller and Michiel van Noort for the initiation of this project, their untiring effort to generate high-quality solar data, for continuously answering my questions, introducing me to the fascinating world of solar observations, and for giving me the opportunity to work independently and to develop my own ideas. I am thankful for everything you taught me. I also thank Prof. Ansgar Reiners for his support, in particular his willingness to act as a referee for this thesis.

Many thanks to Francisco Iglesias, who taught me brilliantly about FSP and reducing data in the beginning phase of my $\mathrm{PhD}$. It was a great pleasure to discuss the theoretical details of (solar) physics with Rafael Manso Sainz - this thesis would not have been what it is without him, in many aspects. Thank you Rafa for patiently answering my questions in detail, also during the writing phase of this thesis.

The SLAM Team is acknowledged for the interesting science meetings, the exchange of ideas and for providing feedback. One special thanks to Ivan Milic, who proofed to be the best RTE teacher. Sudharshan, Ameya, Mayukh, Kamal - thank you for never giving up on my social live and for inspiring discussions. I thank the Rocks-and-Stars Conference and the Solar Polarization Workshop 9 organizing committee members for the great experience of helping to organize a scientific conference.

Moreover, I am grateful to Sonja Schuh for inviting me to apply for the International Max Planck Research School for Solar System Science at the University of Göttingen (IMPRS) and for always providing support with PhD administration. I thank the IMPRS for the funding and the opportunity to travel to conferences and observatories, and the IMPRS students and MPS staff for creating a pleasurable working environment. A special thanks to the motivated IMPRS beach-volleyball group for helping with the stress management. Likewise, I am thanking Rafael Manso Sainz, Ameya Prabhu, and Anna Terbrüggen and especially Nuno Guerreiro for proofreading parts of the thesis. I am grateful to the IRSOL director Michele Bianda and the staff for giving me the freedom to finish this thesis, while already working on another project.

I dedicate this thesis to my family — and my non-academic passion, Ben. 



\section{Curriculum vitae and publications}

\section{Personal information}

Name: Franziska Zeuner

Year of birth: 1989

Work experience

since 2019: IRSOL (Switzerland)

Scientific researcher for absolute high-precision polarimetry

2016-2020: Georg-August-Universität Göttingen (Germany)

PhD thesis under the International Max Planck Research School (IMPRS) on Solar System Science at the Max-Planck-Institut für Sonnensystemforschung (High resolution scattering spectropolarimetry of the quiet solar photosphere)

\section{Education}

2014-2015: Universität Paderborn (Germany)

Scientific research assistant in the working group "Ultra-fast Nanophotonics"

2013-2014: Universität Paderborn (Germany)

Master of Science (Coherent control of localized plasmonic excitations via optical nearfield coupling)

2009-2013: Universität Paderborn (Germany)

Bachelor of Science (Realisation and characterisation of an actively stabilized Michelson-interferometer for coherent control of NIR-fs-pulses)

\section{Publications}

Zeuner, F., Feller, A., Iglesias, F. A., Solanki, S. K.: Detection of spatially structured scattering polarization of Sr I 4607.3 $\AA$ with the Fast Solar Polarimeter - Astronomy \& Astrophysics 619, A179 (2018)

Zeuner, F., Sainz, Manso Sainz, R., Feller, A., van Noort, M., Solanki, S. K., Iglesias, F. A., Reardon, K., Martinez Pillet, V.: Solar disk center shows scattering polarization in the Sr I $4607 \AA ̊$ Aine - The Astrophysical Journal Letters 893, L44 (2020) 